\title{
Ultrasound-based Therapies for the Treatment of Cancer
}

A DISSERTATION PRESENTED TO THE FACULTY OF THE SCHOOL OF ENGINEERING AND APPLIED SCIENCE

UNIVERSITY OF VIRGINIA

IN PARTIAL FULFILLMENT OF THE REQUIREMENTS FOR THE DEGREE

DOCTOR OF PHILOSOPHY IN BIOMEDICAL ENGINEERING

By Kelsie F. Timbie

December

2016 
APPROVAL SHEET

The dissertation

is submitted in partial fulfillment of the requirements

for the degree of

Doctor of Philosophy

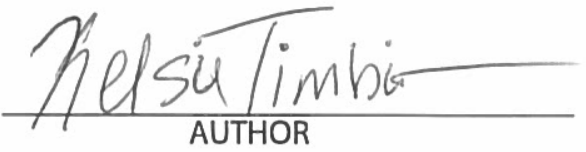

The dissertation has been read and approved by the examining committee:

\begin{tabular}{c} 
Richard Price \\
\hline $\begin{array}{c}\text { Advisor } \\
\text { Kimberly Kelly }\end{array}$ \\
\hline Timothy Bullock \\
\hline Wilson Miller \\
\hline John Hossack \\
\hline Sasha Klibanov
\end{tabular}

Accepted for the School of Engineering and Applied Science:

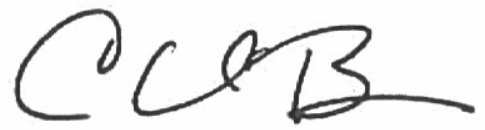

Craig H. Benson, Dean, School of Engineering and Applied Science

December

2016 
I would first of all like to thank Dr.Price for his support, advice and patience over the years. His guidance, knowledge and experience have been invaluable, and I have been privileged to benefit from his talents both in the lab and the classroom.

I thank my committee members, Dr.Kelly, Dr.Bullock, Dr.Miller, Dr.Hossack and Dr.Klibanov for their assistance as well. I particularly thank Dr. Hossack for introducing me to the world of ultrasound physics, and Dr.Klibanov for his lessons on microbubble chemistry and for always being ready with a smile, even when we run into each other at 10:00 pm on a Tuesday in MR5. I am also grateful for Dr.Bullock's ready sense of humor and enthusiasm, which made my introduction to the alphabet soup of immunology bearable. Finally, I am beyond thankful for the endless patience and friendship of Dr.Miller, in providing continual instruction on MR physics for chemical engineers, soldering and life. Thank you for reminding me why I wanted to do this, and for maintaining my sanity through long hours in the MR cave.

I would also like to thank my Price Lab family, past and present, for their support, assistance and solidarity. Caitlin Burke, Josh Meisner, Josh Heuslein, Brian Mead, Colleen Curley, Natasha Sheybani and Katie Gorick - thank you for your friendship and help over the years! To my undergrad team, in particular Cameron Louttit, Lena Badr, Benjamin Campbell and John McMichael - having the opportunity to work with you was a true privilege and one of the highlights of my graduate school experience. You are all rock stars and I expect great things for you all. Finally, I would like to thank Ji Song for her assistance with sectioning, staining, finding obscure lab supplies in mis-labelled drawers and most importantly, her friendship. You made even the rough days bearable.

Many thanks to my 'support staff' as well. Jeremy Gatesman constantly amazes with his ability to nail a tail vein catheterization while cracking a joke, and I thank Gina Wimer and the staff of the Snyder vivarium for always being willing to provide my animals with extra TLC. Aaron Stevens, Jessica Prince and Andrew Buckner - I apologize for those long Fridays, and thank you for your expertise and advice! To the amazing crew at Johns Hopkins - Elizabeth Nance, Clark Zhang, Umara Afzal - I couldn't have done it without you.

Sincerely,

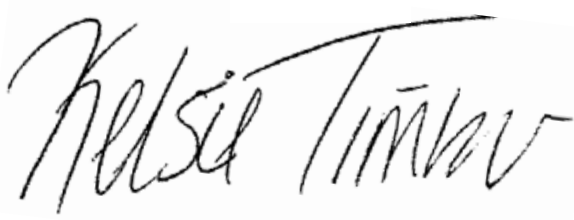


Ultrasound has gained popularity in recent years due to its ever-increasing therapeutic potential. Therapeutic ultrasound is capable of producing a range of bioeffects, such as localized heating or non-thermal disruption of the vasculature. Here, we propose an exploration of two distinct ultrasound-based cancer treatments: transcranial blood-brain barrier disruption (BBBD) for therapeutic nanoparticle delivery to primary brain tumors and immunotherapeutic tumor microenvironment modulation with enhanced drug delivery for melanoma.

\section{Drug delivery to the brain}

The brain has long presented a unique challenge for drug delivery in the form of the blood-brain barrier (BBB). The BBB prevents the vast majority of chemotherapeutics from entering the brain, and most treatment modalities (direct injection, convection enhanced delivery, surgery) are invasive. In BBBD, low intensity ultrasound is focused through the intact skull. Microbubbles (MBs) injected into the blood stream expand and contract in the focal region within the brain, producing mechanical forces that interact with the vessel walls. As a result of these forces, the blood-brain barrier is disrupted in a localized, reversible and non-invasive manner, allowing delivery of systemically administered chemotherapeutics. We determined whether focused ultrasound (FUS) is capable of delivering high specialized "brain-penetrating nanoparticles" (BPNs), designed to diffuse within the brain parenchyma, across the BBB in a rodent model of glioblastoma. First, we defined a safe, repeatable protocol for MR-guided FUS-mediated BBBD in the rat brain based on T1 and T2*-weighted MR images, MR thermometry and histology. Next, detailed analysis of confocal microscopy images taken from treated brains demonstrated that FUS in combination with MBs is capable of delivering $60 \mathrm{~nm}$ fluorescently tagged BPNs across the BBB in normal brain and across the blood-tumor barrier in orthotopic glioblastoma. Finally, we determined that ultrasound-mediated delivery of drug-loaded biodegradable BPNs in an intracranial rat model of glioblastoma produces significant tumor growth control and survival benefit.

\section{Immunomodulation in melanoma}

While there are currently several immune-based therapeutics available for the treatment of melanoma, a large number of patients do not respond to treatment. This is often attributed to a poor pre-treatment antitumor immune response, and it has been postulated that increasing this baseline immune activity may enhance the efficacy of these immunotherapeutics as well as increase the percentage of responders. It has been shown that high-intensity ultrasound generates an increased antitumor immune response, although the mechanisms by which this occurs are poorly understood. We investigated the effects of ultrasound-based immunomodulation in a mouse model of melanoma. First, we developed a low-intensity microbubble-enhanced ultrasound protocol that provided improved tumor growth control and animal survival compared to an FDA-approved immunotherapeutic (anti-PD-1). Next, we demonstrated that 
ultrasound alone significantly improved tumor growth control compared to a combination of ultrasound and anti-PD-1. To better understand the mechanisms controlling ultrasound-mediated tumor growth control, we performed flow cytometry on treated tumors and showed that ultrasound increases the infiltration of regulatory $T$ cells, helper T cells, cytotoxic T cells, natural killer cells and macrophages. To determine which cell type is responsible for ultrasound-mediated tumor growth control, we have designed three experiments: 1) examine the growth of ultrasound vs. untreated tumors in Rag-1 knock out mice (lacking an adaptive immune system) 2) perform flow cytometry on ultrasound treated animals who have received tagged T cells and 3) perform flow cytometry on ultrasound treated animals who have received FTY720 (which prevented T cell trafficking). With these three experiments, we determined that US-mediated tumor growth control is dependent on trafficking of adaptive immune cells. 


\section{TABLE OF CONTENTS}

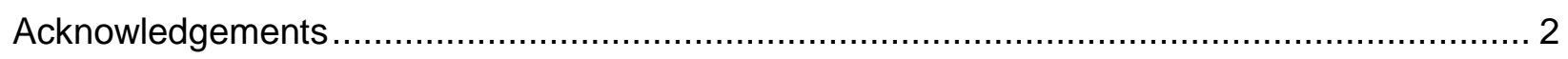

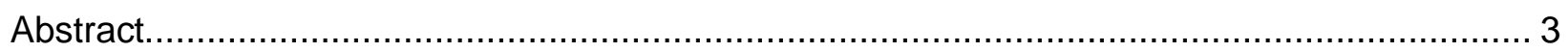

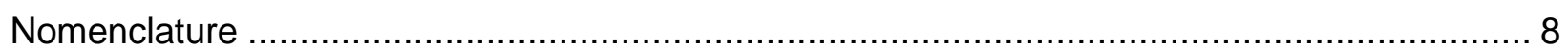

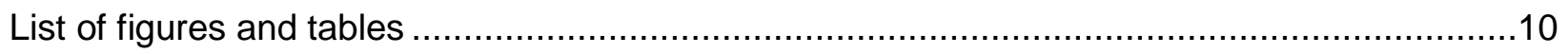

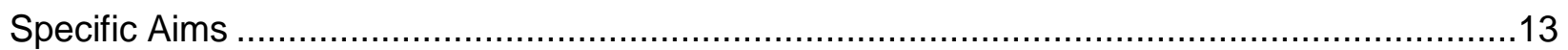

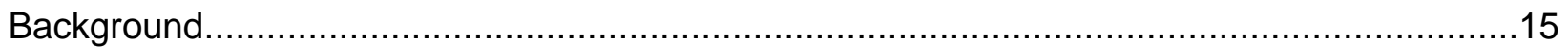

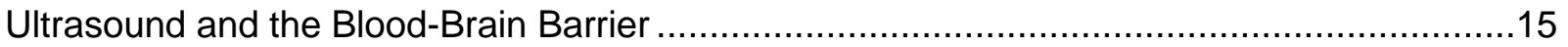

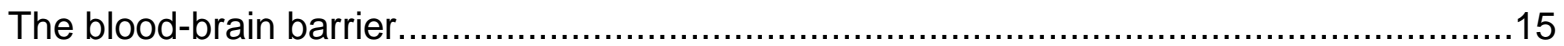

Conventional approaches for bypassing the blood-brain barrier ................................16

Opening the blood-brain barrier with focused ultrasound ......................................17

Safety and monitoring of blood-brain barrier disruption .......................................... 19

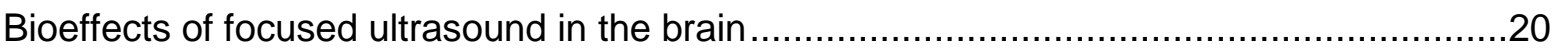

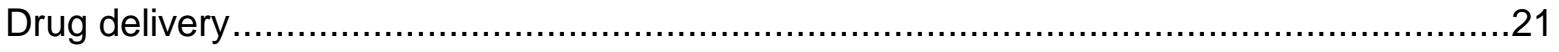

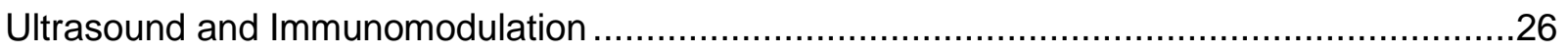

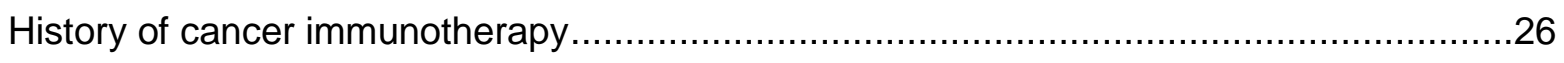

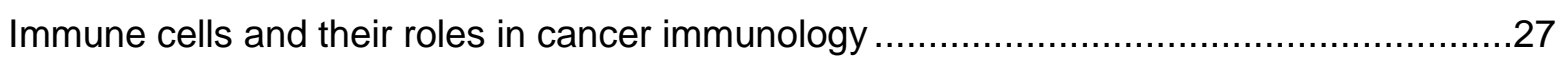

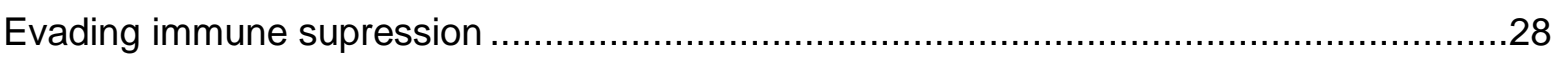

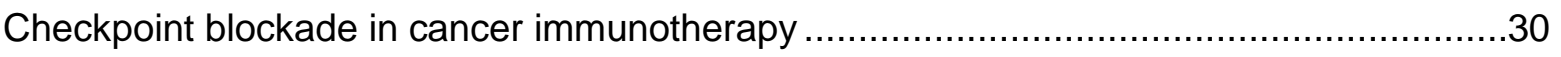

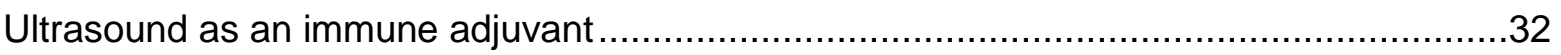

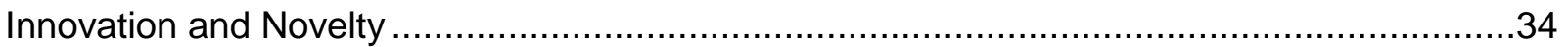

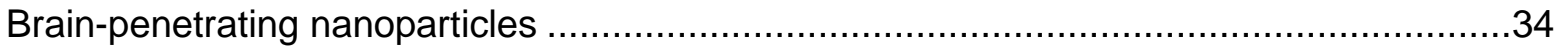

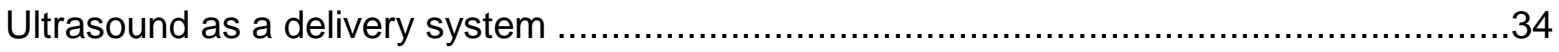

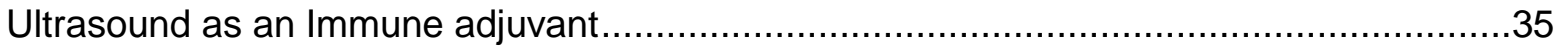

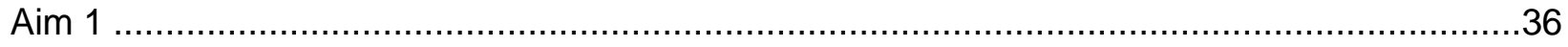

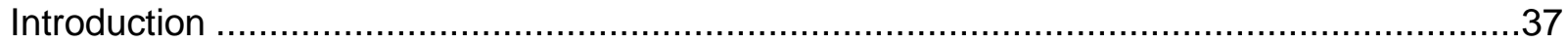

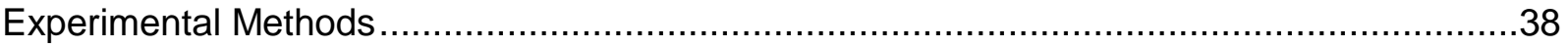

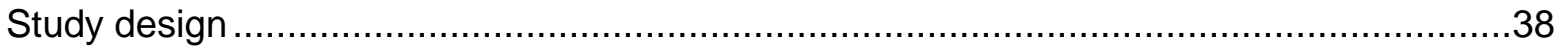

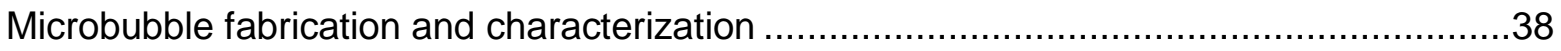

PS-PEG nanoparticle preparation and characterization .........................................38

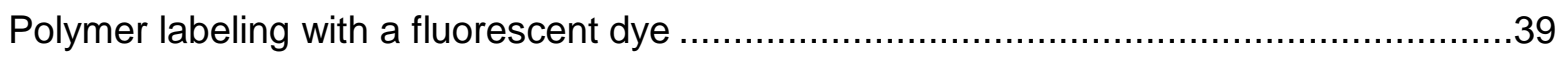

PLGA-PEG nanoparticle Preparation and characterization ......................................39

Nanoparticle diffusion in ex vivo and in vivo rodent brain ......................................40

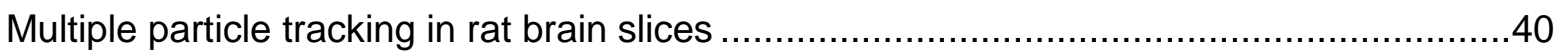

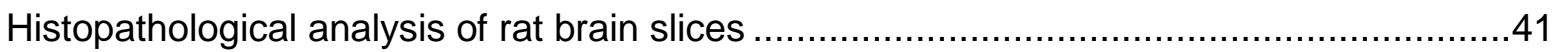




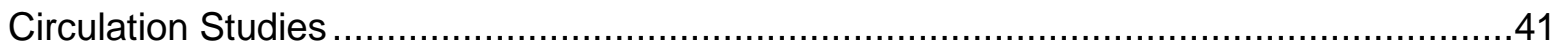

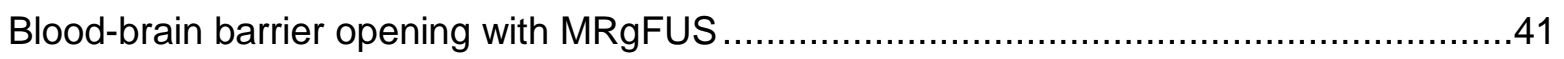

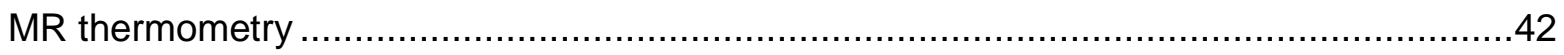

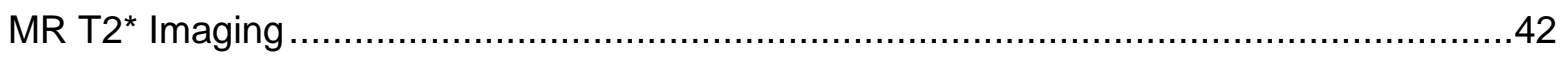

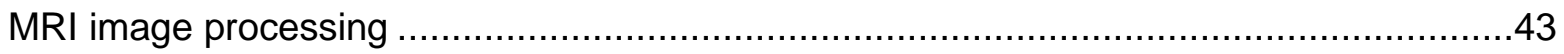

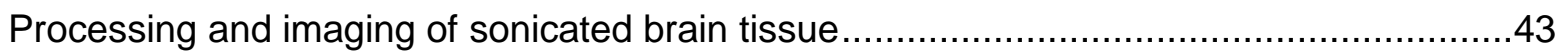

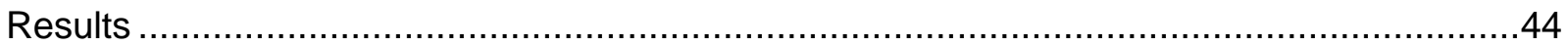

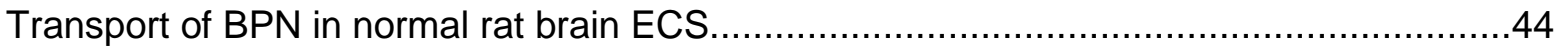

Ex vivo tissue integrity during multiple particle tracking ........................................46

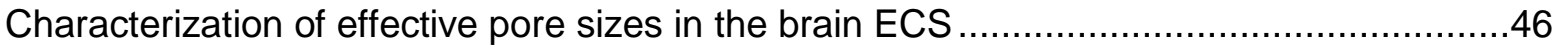

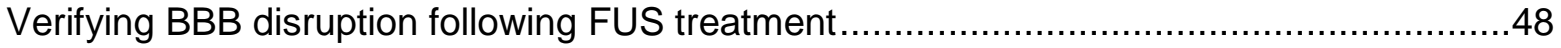

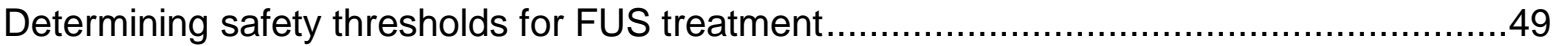

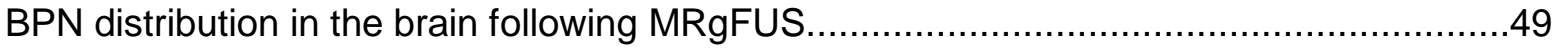

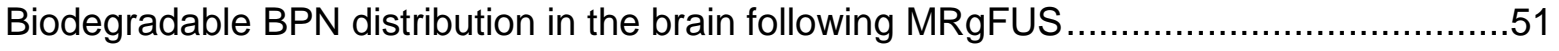

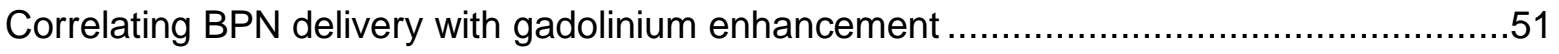

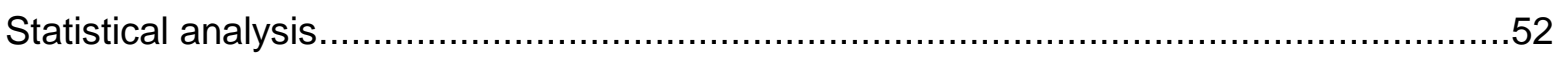

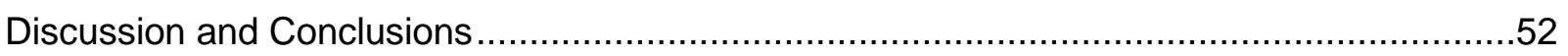

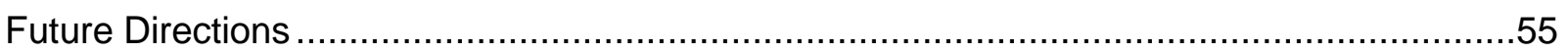

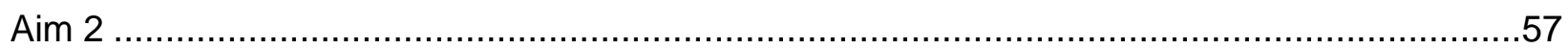

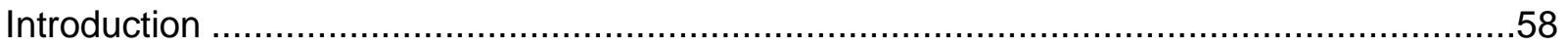

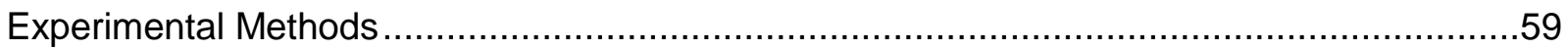

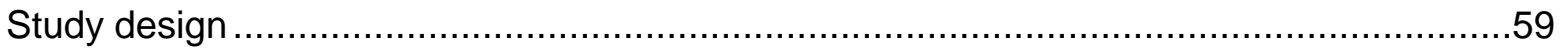

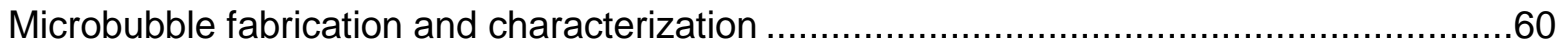

PS-PEG nanoparticle preparation and characterization ..........................................60

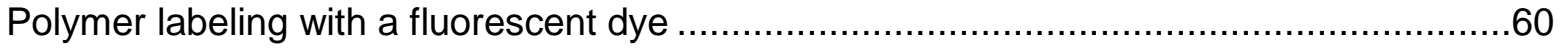

CDDP-BPN nanoparticle fabrication and characterization ......................................60

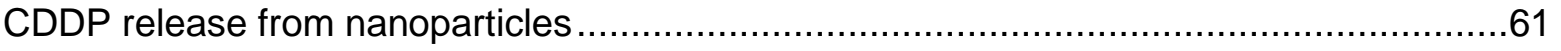

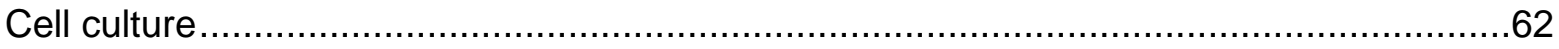

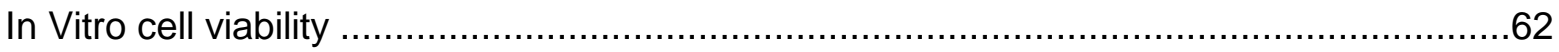

Neocortical slice preparation and multiple particle tracking ....................................62

Fluorescence-based imaging of in vivo NP spread..............................................63

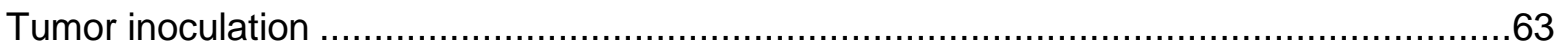

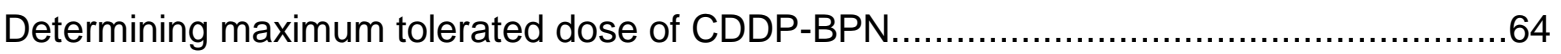

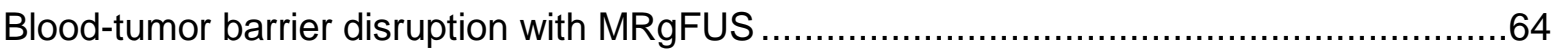

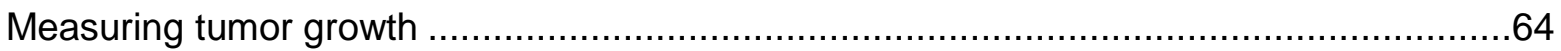




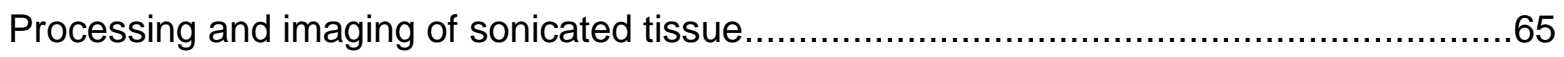

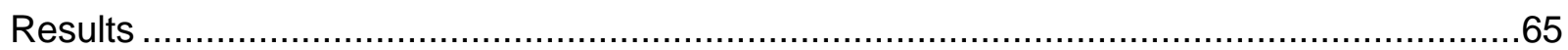

Physicochemical characterization of CDDP nanoparticles ....................................65

In vitro cytotoxicity of CDDP-BPN against rodent glioma cells...................................67

Dense PEGylation enhances CDDP nanoparticle spreading in healthy brain tissue..........67

Dense PEGylation enhances in-vivo distribution of CDDP nanoparticles following

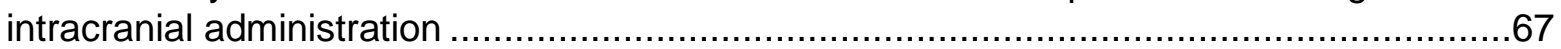

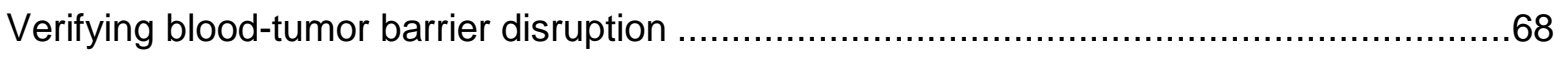

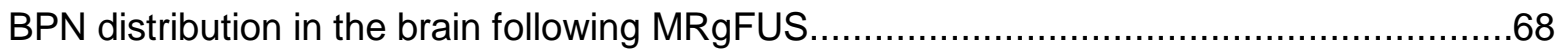

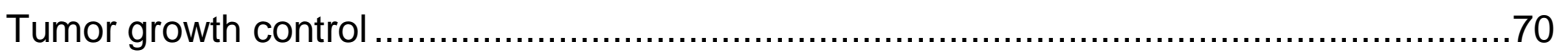

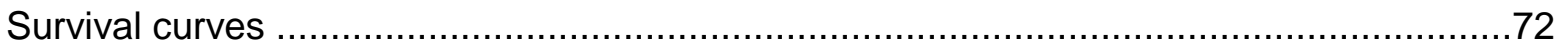

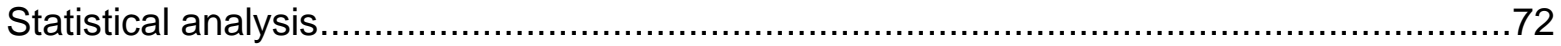

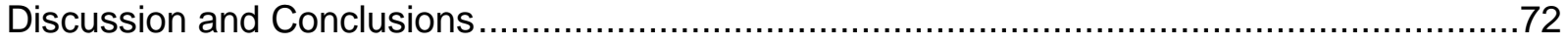

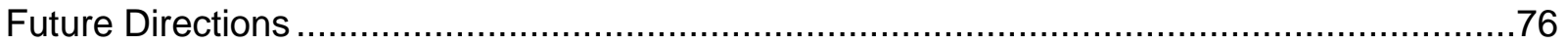

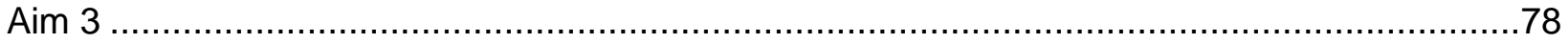

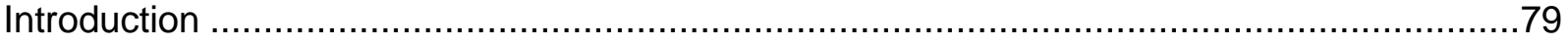

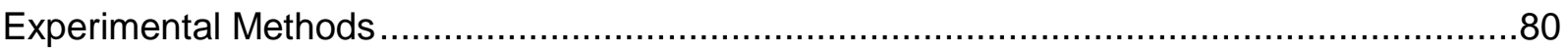

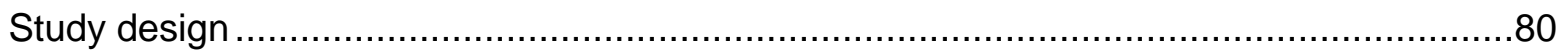

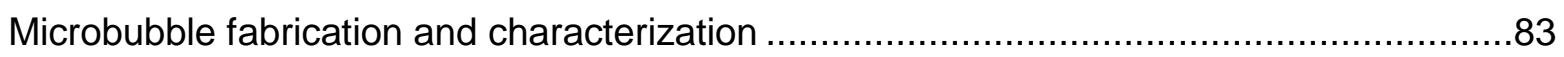

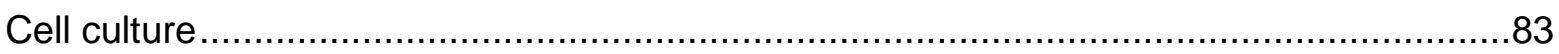

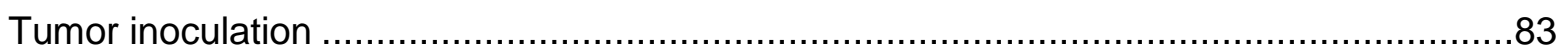

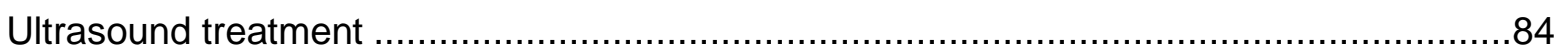

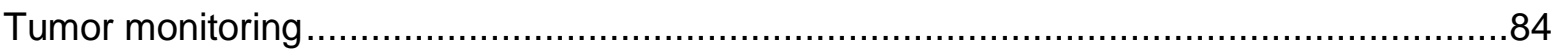

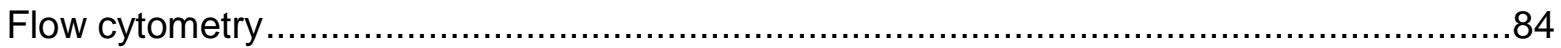

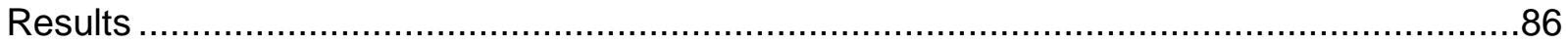

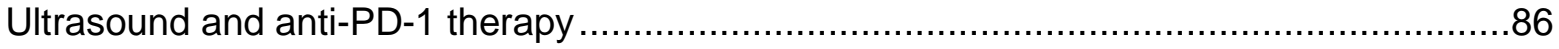

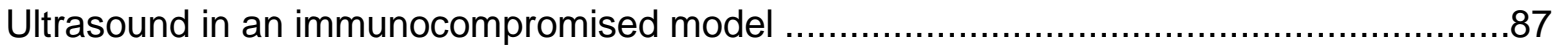

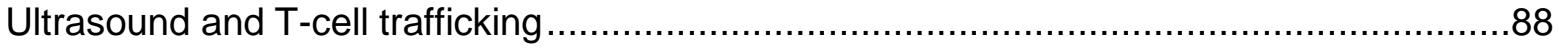

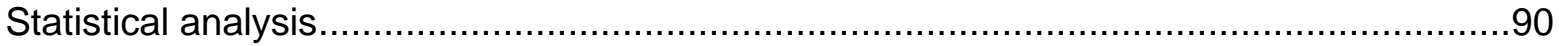

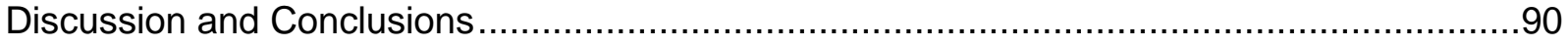

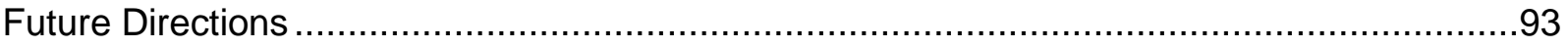

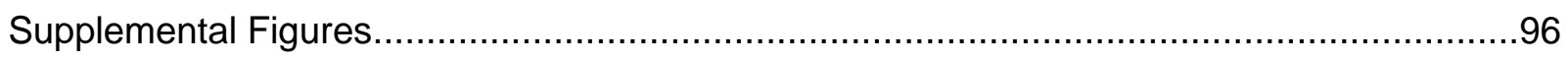

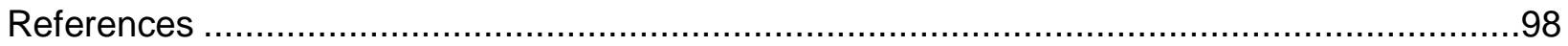


AAS: atomic absorption spectroscopy

ACSF: artificial cerebrospinal fluid

APC: antigen presenting cell

BBB: blood-brain barrier

BPN: brain penetrating nanoparticle

BTB: blood-tumor barrier

CD4 T cell: CD4+ T cell, helper T cell

CD8 T cell: CD8+ T cell, cytotoxic $T$ cell

CDDP: cisplatin

CDDP-BPN: cisplatin-loaded PEGylated polyaspartic acid nanoparticles

CED: convection-enhanced delivery

CNS: central nervous system

CTLA-4: cytotoxic T-lymphocyte associated-protein 4

DC: dendritic cell

ECS: extracellular space

EPR: enhance permeability and retention

FOV: field of view

FUS: focused ultrasound

GBM: glioblastoma multiforme

HIFU: high intensity focused ultrasound

HSP: heat shock protein

MB: microbubble

MRgFUS: MR-guided focused ultrasound

NK: natural killer cell

NP: nanoparticle 
PD-1: programmed death receptor 1

PD-L1: programmed death receptor ligand 1

PEG: polyethylene glycol

PLGA-PEG: PEGylated poly(lactic-co-glycolic acid) nanoparticles

PS-PEG: PEGylated polystyrene nanoparticles

TGI: tumor growth inhibition

TIL: tumor infiltrating lymphocytes

Treg: regulatory $\mathrm{T}$ cell

VA: vascular area 


\section{LIST OF FIGURES AND TABLES}

Figure 1: Blood-brain barrier biology

Figure 2: MR-guided focused ultrasound schematic

Figure 3: Mechanisms of focused ultrasound mediated blood-brain barrier disruption

Figure 4: T cell mediated antitumor response

Figure 5: Cancer immunoediting: elimination, equilibrium and escape

Figure 6: Immune checkpoint blockade in cancer immunotherapy

Figure 7: Highly PEGylated BPNs up to $114 \mathrm{~nm}$ in size diffuse readily in brain tissue

Table 1: Proposed mechanisms of low-intensity ultrasound-induced immune response

Table 1.1: Physicochemical properties of polystyrene nanoparticles and their diffusivity in normal rat brain tissue compared to in ACSF

Figure 1.1: Transport rates of various sized nanoparticles in normal rat brain neocortical slices

Figure 1.2: Prediction of normal brain extracellular spacing based on nanoparticle diffusion

Figure 1.3: FUS-mediated gadolinium delivery and hemorrhage formation in the rat brain are dependent on ultrasound pressure

Figure 1.4: FUS pressure alters particle distribution within the brain

Figure 1.5: MRgFUS-mediated delivery of $60 \mathrm{~nm}$ long circulating brain-penetrating nanoparticles is pressure and time dependent

Figure 1.6: Biodegradable PLGA-PEG NPs distribute in the brain following MRgFUS

Figure 1.7: Gadolinium delivery predicts BPN delivery in MRgFUS-mediated BBB disruption

Table 2.1: Physiochemical properties of CDDP-loaded nanoparticles

Figure 2.1: Physiochemical properties and diffusion behaviors of CDDP-loaded nanoparticles.

Figure 2.2: MRgFUS delivers gadolinium across the blood-tumor and blood-brain barriers 
Figure 2.3: Focused ultrasound increases delivery of brain-penetrating nanoparticles across the BTB and BBB in the $9 \mathrm{~L}$ glioma model

Figure 2.4: Focused ultrasound increases delivery of brain-penetrating nanoparticles across the BTB and BBB in the F98 glioma model

Figure 2.5: Focused ultrasound-mediated delivery of brain-penetrating nanoparticles across the BTB is dependent on vascularity

Figure 2.6: Contrast-enhanced T1 weighted MRI images demonstrate improved tumor growth control when focused ultrasound is combined with cisplatin-loaded brainpenetrating nanoparticles in the F98 rat model of glioma

Figure 2.7: Focused ultrasound improves tumor growth control when combined with cisplatin-loaded brain-penetrating nanoparticles in the F98 rat model of glioma

Figure 2.8: Focused ultrasound improves survival in the F98 rat model of glioma

Figure 3.1: Experimental design: ultrasound and aPD-1 therapy

Figure 3.2: Experimental design: ultrasound in an immunocompromised mouse model

Figure 3.3: Experimental design: ultrasound and t-cell trafficking

Figure 3.4: Schematic of ultrasound pulsing protocol

Table 3.1: Flow cytometry gating strategy

Table 3.2: Flow cytometry antibodies

Figure 3.5: Ultrasound alone improves tumor growth control and survival in a mouse model of melanoma

Figure 3.6: Ultrasound or anti-PD-1 alone improve the tumor immune profile, but this benefit is abolished when they are combined

Figure 3.7: Ultrasound-induced tumor growth control is lost in an immunocompromised mouse model

Figure 3.8: Ultrasound has minimal effects on the tumor immune profile in an immunocompromised mouse model

Figure 3.9: Preventing T-cell trafficking following ultrasound treatment causes loss of tumor growth control

Figure 3.10: Ultrasound treatment increases trafficking of transferred naïve PMEL-1 T cells to the tumor but not does not affect proliferation 
Figure S1: Gating strategy for T cells, natural killer cells, dendritic cells and macrophages

Figure S2: Gating strategy for M1/non-M1 macrophages 
As cancer incidence rises and treatments become more complex, methods to improve drug delivery and efficacy are gaining appeal. Ultrasound is a multifunctional treatment platform that can increase both the delivery ${ }^{1,2}$ and efficacy ${ }^{3}$ of therapeutics. Ultrasound is FDA approved for therapeutic applications ${ }^{4}$ and is capable of being combined with existing ${ }^{5}$ and experimental ${ }^{6}$ therapies, creating a low barrier for clinical translation and offering great potential for improvements in therapeutic outcomes. Here, we investigate two different ultrasound-mediated cancer treatments: delivery of drug loaded biodegradable polymeric nanoparticles across the BBB using FUS in an intracranial glioma model and enhancement of $\mathrm{T}$ cell infiltration in a subcutaneous murine melanoma model.

Drug delivery to the brain: The BBB prevents the vast majority of therapeutics from reaching the brain ${ }^{7}$, greatly complicating the treatment of brain tumors. While the vasculature in the core of the tumor may be heterogeneously permeable ${ }^{8}$, additional constraints such as increased interstitial pressure within the tumor ${ }^{8}$, a nanoporous and bioadhesive parenchyma ${ }^{9}$, and the presence of infiltrating tumor cells beyond the tumor core $^{10}$ make the systemic delivery of chemotherapies ineffective. Transcranial focused ultrasound (FUS) and intravenously administered microbubbles (MBs) produce localized, non-invasive, and reversible BBBD to permit the delivery of systemically administered therapeutics both in and around the tumor ${ }^{11}$. While FUS has been utilized in combination with traditional therapies ${ }^{12}$, polymeric drug delivery systems offer several advantages $^{13}$, and their potential when used in combination with FUS has yet to be determined. Here, we determine the utility of FUS-mediated delivery of unique, highly specialized "brain-penetrating nanoparticles" (BPNs) designed for diffusion within the brain.

\section{Specific Aim 1: To design an MR-guided focused ultrasound protocol for the} delivery of $60 \mathrm{~nm}$, brain-penetrating nanoparticles across the blood-brain barrier in the normal rat brain. Polymeric nanoparticles large enough to carry an efficacious drug load are on the order of $100 \mathrm{~nm}^{14}$, much larger than the small molecule drugs that have been delivered using FUS. Since infiltrating tumor cells are frequently found in areas with normal BBB function ${ }^{10}$, it is important to assess FUS's delivery capability in the healthy brain. MR-guided FUS and MBs and will be used to disrupt the BBB in the presence of circulating BPNs. Contrast-enhanced and T2*-weighted MR images, as well as MR thermometry, will be used to assess protocol safety. BPN delivery and distribution will be assessed using confocal microscopy.

\section{Specific Aim 2: To develop an MR-guided focused ultrasound protocol that} increases the delivery and efficacy of biodegradable, drug-loaded brain penetrating nanoparticles in a rat model of glioblastoma. While FUS is capable of improving drug delivery to tumors, it is unclear whether the delivery of $60 \mathrm{~nm}$, systemically administered drug loaded polymeric nanoparticles will provide effective tumor growth control. MR-guided FUS and MBs will be used to disrupt the blood-tumor barrier following intravenous injection of drug-loaded BPNs. Tumor growth will be monitored using contrast-enhanced MRI. 
Immunomodulation in melanoma: Survival rates for advanced melanoma have remained static for many years ${ }^{15}$. Melanomas' ability to escape immune surveillance is credited with this poor prognosis ${ }^{16}$. While newly developed immune-based therapies can produce dramatic improvements in tumor regression and patient survival, only a small percentage of patients respond to therapy ${ }^{17}$, primarily due to poor $\mathrm{T}$ cell infiltration of the tumor. Ultrasound can be used to elicit an antitumor immune response ${ }^{18}$ and may be capable of increasing baseline $T$ cell infiltration to improve patient response to immunotherapeutics. Here, we investigate a combination of ultrasound and anti-PD-1 therapy.

Specific Aim 3: To determine whether the activation of microbubbles with ultrasound can enhance the adaptive antitumor immune response. Unfocused ultrasound (US) can produce improvements in tumor growth control, but the mechanism is unclear. US-mediated improvement in T cell infiltration may greatly improve the efficacy of T-cell based therapies, such as anti-PD-1 treatment, and a better understanding of US-induced immunomodulation may allow us to utilize this technique with greater efficacy and predict how it will interact with other therapies. Immunecompromised mice will be used to distinguish the effects of $B$ and T cells in treated tumors, and adoptive $T$ cell transfer with tagged $T$ cells will permit us to measure the contribution of T cell trafficking. Treatment with FTY720, which prevents T cell trafficking, will allow us to determine the rate of $T$ cell proliferation within ultrasound treated tumors. 
Parts of this chapter have been published in Timbie KF, Mead BP, Price RJ. Drug and gene delivery across the blood-brain barrier with focused ultrasound. J Control Release. September 2015.

\section{ULTRASOUND AND THE BLOOD-BRAIN BARRIER}

Many diseases of the central nervous system (CNS) present tremendous challenges for clinicians. Both primary and metastatic brain tumors carry dismal survival rates ${ }^{10,19}$, and the increasing age of the population in the developed world has created a dramatic increase 20 in the number of people living with age-related neurodegenerative diseases like dementia, Alzheimer's and Parkinson's. Additionally, nearly $20 \%$ of the adult population ${ }^{21}$ experiences the debilitating effects of a mental illness like obsessivecompulsive disorder or clinical depression each year, generating over \$44 billion in lost productivity in the US alone ${ }^{22}$. The commonality in this wide range of CNS disorders is the inherent difficulty of treatment. The blood-brain barrier (BBB) provides excellent protection for the body's most privileged organ, preventing the vast majority of molecules in circulation from entering brain tissue. However, because of this, the BBB also presents a significant challenge for CNS treatments, as systemic therapies are rarely capable of crossing the BBB. Recently, the ability of focused ultrasound (FUS) in conjunction with microbubbles (MBs) to facilitate the noninvasive, localized, and reversible opening of the BBB has led to the emergence of this technology as a viable new option for delivering therapeutics to the CNS.

\section{THE BLOOD-BRAIN BARRIER}

The BBB provides a formidable obstacle for drug delivery in the brain (Figure 1). Through a unique combination of transmembrane proteins and tightly regulated channels not seen elsewhere in the body, the BBB prevents nearly $100 \%$ of large molecule $(>500 \mathrm{Da}$ ) drugs, including recombinant proteins and antibodies, and $98 \%$ of small molecule drugs, from passing into the brain ${ }^{23}$. Lipid soluble small molecule drugs may cross the BBB if they are capable of diffusing through the endothelial cell membrane itself ${ }^{23}$, but few drugs fall into this category. The BBB's remarkable exclusionary capability is attributed to tight junctions that join the endothelial cells lining the vasculature throughout the brain ${ }^{7}$. Tight junctions are comprised of several proteins, including various claudins, occludins, junctional adhesion molecules, and cadherins, which function to prevent molecules from passively diffusing between cells and out of the vasculature ${ }^{7}$. Rather, small molecules must pass through the endothelial cells themselves, either through diffusion (for lipid soluble molecules) or active transport (most nutrients and other substances necessary for normal brain function) ${ }^{7}$. Furthermore, if a certain molecule does manage to pass through the endothelial cell layer, the basement membrane provides an additional barrier to diffusion. Simply stated, nature's best defense against infection significantly hinders our ability to treat diseases of the CNS by preventing drug delivery to the brain. 


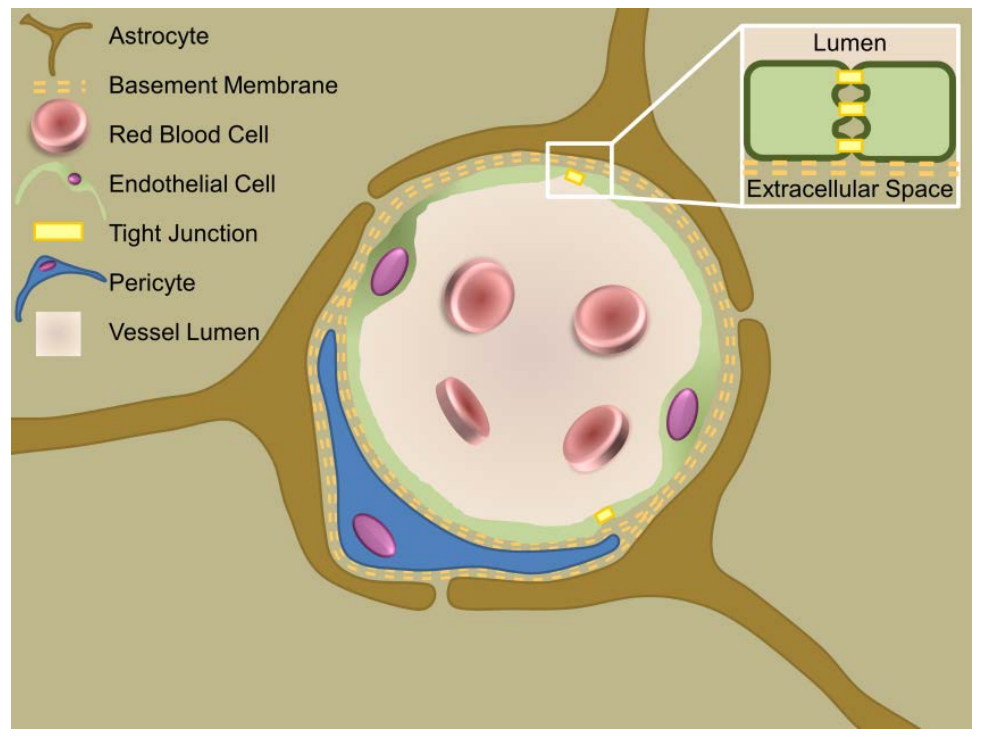

\section{Figure 1. Blood-Brain Barrier}

Biology. The blood-brain barrier presents a major obstacle to therapeutic delivery in the central nervous system. It is comprised of unusually abundant and structurally unique tight junctions between the vascular endothelial cells and a thick basement membrane. Regulation via astrocytes and pericytes maintain this barrier, preventing the passage of the vast majority of therapeutics. ${ }^{270}$

\section{CONVENTIONAL APPROACHES FOR BYPASSING THE BLOOD-BRAIN} BARRIER

Given the central role of the BBB in limiting drug and gene delivery to the CNS, numerous methods have been developed to bypass this barrier. For example, specific viruses or nanoparticles (NPs) with BBB-targeting ligands can cross the BBB after systemic administration ${ }^{24}$. However, in order to achieve effective concentrations in the brain, they must be administered in doses which are associated with adverse effects in peripheral organs ${ }^{25}$. For this reason, the majority of preclinical and clinical studies have used direct intracranial administration as a strategy to locally increase therapeutic concentration without off-target effects. Specific brain regions can be accessed with needles or catheters and more recent strategies have utilized fluid convection to enhance distribution of therapeutics in the brain ${ }^{26}$. By maintaining bulk flow with hydrostatic pressure differentials, convection enhanced delivery has demonstrated marked improvement over conventional direct intracranial injection methodologies 27,28 . Unfortunately, despite promising results for direct injection in several preclinical and clinical trials ${ }^{29-33}$, these strategies are risky and surgical complications have hindered widespread adoption. Furthermore, macromolecular agents require long dissemination times and typically cannot spread beyond a few millimeters ${ }^{27}$. Indeed, the invasive nature of strategies like intracranial injections is not compatible with drugs that need to be dosed repeatedly.

In order to reduce risks associated with direct injection, less-invasive strategies to enhance therapeutic delivery across the BBB have been developed. These include intranasal administration and chemical disruption of the BBB by intra-arterial infusion of the osmotic agent mannitol ${ }^{34}$ or vasodilators ${ }^{35-37}$. Intranasal administration permits transport to the brain through perineural or perivascular channels ${ }^{38}$. While intranasal drug delivery is non-invasive and obviates peripheral side effects associated with intravenous administration, it is limited by poor absorption across the nasal epithelium, inconsistent delivery efficiency and poor localization ${ }^{38,39}$. Similarly, mannitol infusions 
lead to global BBB disruption, causing non-specific uptake and potentiating adverse offtarget effects. Infusion of mannitol into the carotid artery leads to an osmotic-driven movement of fluid out of endothelial cells ${ }^{40}$, shrinking them and leading to fenestration of cerebral vessels. While disruption of the BBB with mannitol is reasonably safe, therapeutic delivery is inconsistent with up to 10 -fold variations in drug concentrations ${ }^{41}$.

\section{OPENING THE BLOOD-BRAIN BARRIER WITH FOCUSED} ULTRASOUND

FUS has the advantage of being the only modality capable of achieving noninvasive, safe, repeated, and targeted BBB disruption to enhance drug or gene delivery to the CNS. With the advent of MR-compatible transducers with sub-millimeter precision, it is now possible to apply image-guided transcranial FUS to the human brain 42-45 in an extremely localized manner, greatly reducing the risk of off-target effects. FUS treatments can be performed on awake patients, eliminating the need for general anesthetic and permitting real-time patient feedback. Importantly, MR and integrated passive cavitation detection (PCD) facilitate real-time intraoperative treatment monitoring, while post-treatment MR imaging allows confirmation of treatment success ${ }^{46-49}$ and safety ${ }^{50,51}$. The development of transcranial FUS has been a long process. Groundbreaking research by the Fry brothers performed over 50 years ago demonstrated that ultrasound could produce bioeffects in the human brain ${ }^{52}$. However, it wasn't until recent technological advances were made in both ultrasound and MRI that the field experienced a surge in interest. In the past ten years, there has been an increase in the number of papers investigating the potential applications of ultrasound in the brain.

Ultrasound is, at its most basic, a pressure wave. As the wave passes through the tissue, the tissue experiences alternating periods of high pressure (compression) and low pressure (rarefaction). Ultrasound can be applied in a continuous fashion, common in treatments that require heat deposition, or in a pulsed manner, which is utilized for blood-brain barrier disruption (BBBD). Focusing the ultrasound beam (i.e. FUS) provides high spatial accuracy (less than $1 \mathrm{~mm}$ resolution in some cases) and localizes bioeffects. However, reflection and diffraction of the ultrasound wave at material interfaces (i.e. skull-tissue interface) can distort the focus and decrease the energy delivered at the target. While the favorable skull geometry of rats or mice allows the use of single-element transducers in pre-clinical trials (Figure 2), the far more complex topography of the human skull requires the use of a multi-element array with phasecorrection software to re-focus the ultrasound beam as it passes through the skull. There are many combinations of FUS parameters (frequency, pressure, pulsing protocol) suitable for BBBD, but lower frequencies $(\leq 1.0 \mathrm{MHz})$ experience less attenuation and distortion by the skull. 


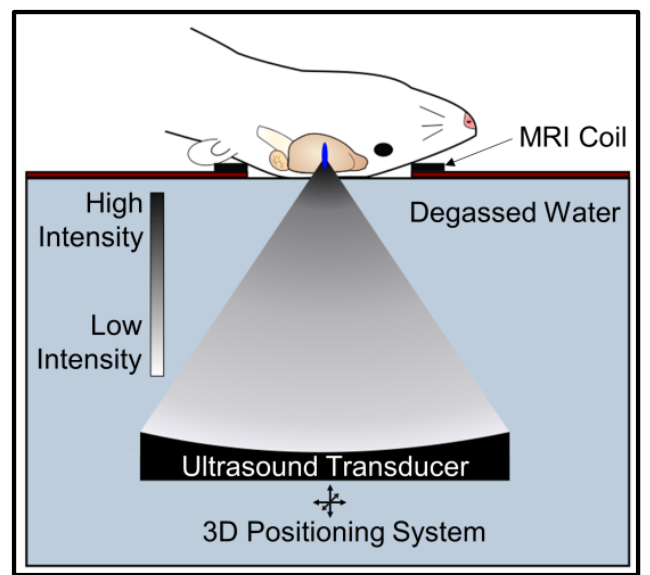

Figure 2. Transcranial FUS with

microbubbles is the only modality

capable of achieving non-invasive, safe, repeated and targeted $B B B$ disruption,

leading to improved drug or gene

delivery to the brain. Pre-clinical FUS

studies in animals including mice and rats

permit use of a single-element FUS

transducer, due to favorable skull geometry.

FUS can be guided with MR imaging and is

capable of sub-millimeter resolution allowing

precise targeting of structures in the CNS

with minimal off-target effects. ${ }^{270}$

Transcranial FUS is typically applied in conjunction with intravenously administered MBs to effect BBBD. MBs are small (1-10 $\mu \mathrm{m})$ lipid or protein shelled bubbles filled with an inert gas, most commonly a perfluorocarbon and are FDA-approved as a contrast agent during ultrasound imaging. Importantly, circulating MBs reduce the acoustic energy required to open the BBB by two orders of magnitude and confine mechanical effects to the vasculature ${ }^{53}$. This permits the use of low pressure FUS and virtually eliminates any concerns about skull heating during treatment. Extended off-time (low duty cycle) between FUS pulses allows MB reperfusion and thermal dissipation at the focus. At the lower ultrasonic pressures used for BBBD, MBs oscillate stably in the FUS field, expanding during rarefaction and contracting during compression, producing mechanical shear forces ${ }^{54}$ and microstreaming ${ }^{55}$ effects which act on the vessel wall. This behavior domain, called stable oscillation, is preferred for BBBD as its effects are more predictable. In contrast, at higher acoustic pressures, MBs experience unstable oscillations and eventually collapse inward, producing elevated local temperatures and high-pressure jet streams in a process termed inertial cavitation. While FUS-MB induced BBBD occurs in both regimes, inertial cavitation is more violent and is generally avoided for applications in healthy brain tissue. However, it may find use in diseased tissue, or for the delivery of very large $(\sim 100 \mathrm{~nm})$ therapeutics, when the potential benefits outweigh the risks.

A collection of in vitro and ex vivo work ${ }^{56-58}$ has demonstrated that the mechanical forces exerted by stably oscillating MBs cause vessel distension and invagination, as well as changes in the endothelial cells' cytoskeletons and cell-cell interactions (Figure 3). Together, these effects produce BBBD via three mechanisms: disruption of tight junctions, induction of transcytosis, and sonoporation of the vascular endothelium. Work using transmission electron microscopy imaging ${ }^{59}$ has demonstrated both a reduction and altered distribution of claudins 1 and 5, occludin, and ZO-1 after FUS exposure. Most notably, tight junction proteins were no longer clustered along the edges of endothelial cells, suggesting that they were no longer contributing to tight junction complexes. Furthermore, penetration of horse radish peroxidase between endothelial cells was evident, demonstrating that tight junctions were no longer sealing paracellular pathways from the vasculature to the brain parenchyma. In addition to this paracellular pathway, horseradish peroxidase was also taken up by the vascular endothelial cells after sonication. Later work ${ }^{60-62}$ demonstrated increased expression of caveolin-1 in the vascular endothelium after sonication, identifying caveoli as the most likely transcytotic 
pathway. A unique approach using two-photon microscopy provided further support for both paracellular and transcellular pathways. Here, it was noted that dextrans crossed the BBB after FUS via either a fast or slow mechanism (i.e. less/greater than $10 \mathrm{~min}$ ) and postulated that the fast and slow pathways were most likely paracellular (tight junction disruption) and transcellular (increased transcytosis and sonoporation), respectively ${ }^{63}$. Interestingly, $70 \mathrm{kDa}$ dextrans appeared to have a higher pressure threshold for BBB crossing than smaller 10kDa dextrans, in agreement with other studies indicating that the extent of BBBD (particularly the size of junctional clefts) is related to pressure ${ }^{64}$. Furthermore, smaller vessels (i.e. less than $\sim 25-30 \mu \mathrm{m}$ ) were significantly more likely to be disrupted by FUS ${ }^{2}$ than larger vessels, and fast leakage ${ }^{63}$ (i.e. paracellular) was the dominant mode of transport in these vessels. This difference is attributed to the interactions between MBs and the vessel wall - in smaller vessels, oscillating MBs are more likely to come in close contact with the vessel wall, generating larger circumferential stresses than in larger vessels. Going forward, achieving a better understanding of the dynamics of these transport pathways will be critical for enabling more predictable BBBD, especially with the increased use of larger therapeutics such as antibodies, liposomes and polymer nanoparticles.

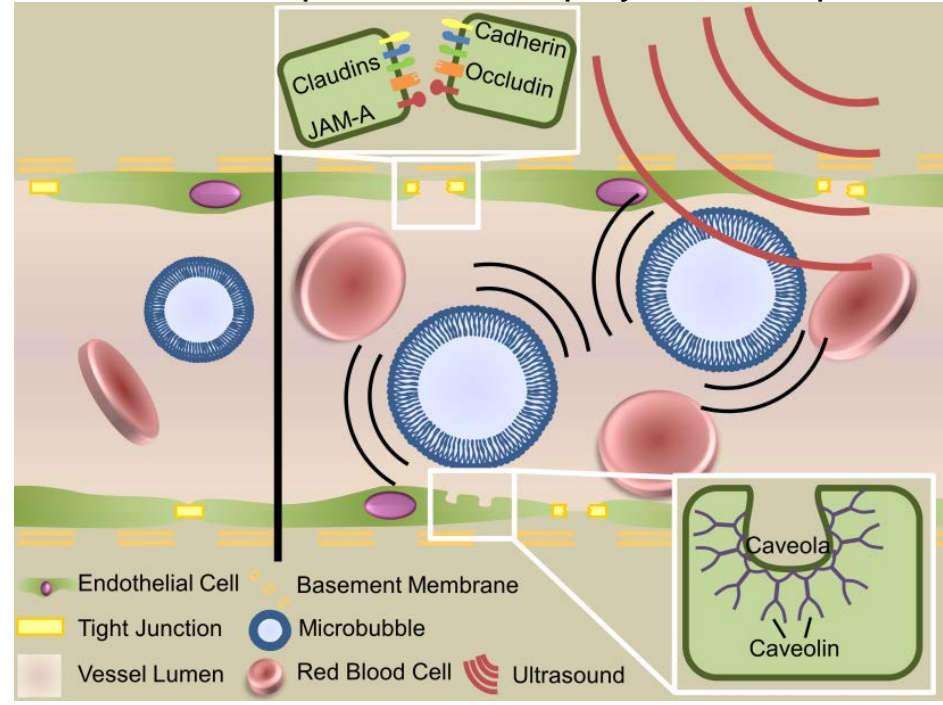

Figure 3. Mechanisms of
focused ultrasound
mediated blood-brain
barrier disruption.
Circulating microbubbles
oscillate in the ultrasonic
field, producing forces that
act on the vessel wall to
generate three bioeffects
that permit transport
across the blood-brain
barrier: disruption of tight
juctions, sonoporation of
the vascular endothelial
cells and upregulation of
transcytosis. ${ }^{270}$

SAFETY AND MONITORING OF BLOOD-BRAIN BARRIER DISRUPTION

While it is well known that driving MBs into inertial cavitation with high acoustic pressures can lead to irreversible capillary damage and the leakage of blood across the $\mathrm{BBB}^{65}$, thresholds have been established wherein BBBD can be achieved without toxicity or damage. BBBD is transient and, depending on acoustic pressure ${ }^{66}$, barrier function is typically restored within 4-6 hours after treatment $59,67,68$. Importantly, no motor or acuity deficits were found after repeated BBBD procedures with FUS at numerous targets in monkeys ${ }^{69,70}$. Interestingly, some of these monkeys had T2* hypointensities in post-FUS MR imaging, indicating minor red blood cell accumulation; however, these minor capillary leakages did not lead to any changes in visual acuity or motor skills. Furthermore, it is important to emphasize that, even in the rare occurrence of erythrocyte extravasation at the lower pressures used in these studies, no apoptotic bodies were found and cognitive function of the animals was not impaired ${ }^{69}$. These 
findings are in agreement with other studies suggesting that minor capillary damage and red blood cell extravasation is not expected to lead to long term effects ${ }^{48,71}$. Indeed, it is possible that such damage would be acceptable in treatment of debilitating or lifethreatening neurological diseases. It is important to further emphasize that FUS-related safety issues would be minor compared to those of other treatment strategies like intracranial injection, which can lead to extensive damage along the needle tract, or even non-invasive treatments like gamma knife radiosurgery ${ }^{72,73}$.

While FUS-mediated BBBD has been shown to be safe in numerous animal models, intraoperative monitoring with passive cavitation detection or MR imaging further reduces chances of aberrant FUS treatments. Passive cavitation detection (PCD) allows real-time assessment of MB cavitation ${ }^{74}$. While stable cavitation is most likely responsible for reversible $B B B D^{65,75}$, inertial cavitation has been linked with tissue damage ${ }^{76}$. Importantly, acquired PCD intensity is well correlated with BBBD ${ }^{65,75}$. PCD non-invasively detects the acoustic signatures resulting from MB oscillations, and can distinguish between stable oscillations and MB collapse. ${ }^{77}$. Stably oscillating MBs emit harmonic, subharmonic or ultraharmonic frequency acoustic emissions, whereas collapsing MBs emit broadband acoustic signals ${ }^{78,79}$. PCD has been used to ensure safe FUS settings in several large animal BBBD models ${ }^{69,80}$ and systems are currently in development that will allow fully automatic feedback to control FUS sonications ${ }^{81}$. Once the FUS treatment is complete, MR imaging sequences including T2* and susceptibility weighted imaging (SWI) are sensitive tools that can be used to detect blood products present in tissue $e^{50,67,82}$ and have been shown to be sensitive to measure even minor capillary damage ${ }^{69}$.

\section{BIOEFFECTS OF FOCUSED ULTRASOUND IN THE BRAIN}

This dissertation is focused on the delivery of drugs across the BBB with FUS; however, it is also important to note that recent advances in our understanding of biological responses to FUS have potentiated novel approaches to treatments of brain disease, even without administration of pharmacological agents. For example, BBBD with FUS alone in a mouse model of Alzheimer's disease led to a significant reduction of plaque burden four days after a single treatment ${ }^{83}$. The authors showed that plaque reduction was linked to significant enhancement of endogenous antibodies bound to the $A \beta$ plaque as well as to activation of microglia and astrocytes in the FUS-treated region. Additionally, five successive treatments with ultrasound were shown to lead to further plaque clearance and improved subject performance on several memory tasks ${ }^{84}$. Nonthermal FUS has been used in other disease models to enhance the body's antitumor immune response with great success ${ }^{85}$, and it has been postulated that FUS-mediated BBBD may provide a similar benefit in brain tumors by increasing endogenous antibody delivery and reducing barriers to immune cell migration in the brain parenchyma ${ }^{83,86}$. In addition to alterations in immune function and cell behavior within the brain, FUSmediated BBBD has been shown to enhance neurogenesis ${ }^{87}$, which is attributed to the demonstrated upregulation of $\mathrm{BDNF}^{88}$ and Akt ${ }^{89}$ after FUS. This observation has led to suggestions that FUS could be used as a non-invasive alternative to deep brain stimulation for treatment of depression ${ }^{90}$. Importantly, low intensity FUS has also shown 
ability to transiently stimulate neurons in both animals ${ }^{88,91-94}$ and humans ${ }^{95,96}$, and has the ability to elicit acute sensory responses in the fingers and hands ${ }^{96}$, potentially allowing non-invasive brain mapping. Ultimately, we believe that FUS will permit several therapeutic options in the CNS, beyond those dependent upon drug and/or gene delivery across the BBB.

\section{DRUG DELIVERY}

FUS-mediated BBBD permits the delivery of a wide range of therapeutics, and improves the efficacy and safety profile of the few drugs which can cross the BBB by reducing the required systemic dose. FUS has demonstrated remarkable ability to deliver a wide range of payloads, including small molecule drugs[79,85], $150 \mathrm{kDa}$ antibodies ${ }^{99}$, recombinant proteins ${ }^{100}$ and even $\sim 100 \mathrm{~nm}$ liposomal drug vehicles ${ }^{12,101}$. As FUS technology has improved over the last decade, work has progressed from the delivery of free small molecule drugs $[79,85]$ such as temozolomide to larger plaquebinding antibodies ${ }^{99}$ and $\sim 100 \mathrm{~nm}$ liposomal drug vehicles ${ }^{12,101}$. In addition, functionalized MBs ${ }^{102}$, targeting moieties ${ }^{103}$ and two-step processes like boron neutron capture therapy ${ }^{104}$ have also been investigated in conjunction with FUS to further enhance delivery efficiency into the CNS. Here, we review work demonstrating the delivery of systemically administered small molecule-, recombinant protein- and antibody-based therapeutics in the brain using FUS-mediated BBBD.

UNENCAPSULATED DRUG

Temozolomide (TMZ), a small molecule drug, is currently part of the recommended approach to the clinical treatment of glioblastoma multiforme (GBM), and many clinical trials continue to test various TMZ dosing regimens as well as drug combinations ${ }^{105-108}$. Although TMZ has produced moderate improvements in patient survival, GBM is notorious for tumor recurrence after surgical resection because infiltrating tumor cells, which are protected from systemic drug delivery by the BBB, inevitably remain after surgery. Thus, it has been hypothesized that the delivery of TMZ via FUS-mediated BBBD may improve patient outcomes by providing drug delivery to these "protected" infiltrating cells ${ }^{97,109}$. In support of this hypothesis, in a rat model of GBM, BBBD in combination with orally administered medium dose TMZ significantly increased survival (IST median $=15 \%$ compared to controls) and controlled tumor growth as well as high dose TMZ alone ${ }^{109}$. A study using the U87 glioma model in mice further demonstrated that FUS treatment improves tumor growth control and survival over TMZ alone across a range of TMZ doses (IST median=111\% compared to control for highest dose TMZ + FUS), although the benefit is most pronounced for low dose regimens ${ }^{97}$. This effect appeared to be due to an increase in TMZ concentration and retention time (2.7- and 1.5-fold, respectively) in sonicated tissue ${ }^{97}$. These studies demonstrate that BBBD with FUS can enhance the efficacy of even "gold standard" drugs in GBM.

While able to cross the BBB, carmustine (bis-chloroethylnitrosourea, $\mathrm{BCNU}$ ) is another small molecule chemotherapeutic drug whose effectiveness could be enhanced by improved local delivery, as it is highly toxic and degrades within 15 minutes. BCNUloaded polifeprosan 20 Gliadel wafers were one of the first uses of biodegradable polymers for drug delivery in humans ${ }^{110-113}$, representing a unique solution to the problems posed by BCNU. Disappointingly, Gliadel produced only mild improvements in 
patient survival (2.3 months compared to placebo), and is now only recommended for patients with fully resectable tumors ${ }^{113}$. Given these limitations, FUS-mediated delivery has been hypothesized to provide similar benefits. To this end, intravenous BCNU has been administered in conjunction with FUS-mediated BBBD, which doubled BCNU deposition in a C6 glioma model. It was shown that this combined treatment provides better tumor growth control and improved animal survival (IST median $=86 \%$ compared to control) ${ }^{114}$. This study also indicates that it may be possible to decrease the intravenous dose administered while maintaining therapeutically relevant drug concentrations in the brain, thus reducing systemic toxicity effects without the need for surgery. Ultimately, it is evident that BBBD with FUS can improve outcomes, even when used in conjunction with drugs that are able to cross the BBB, by increasing local drug concentrations and decreasing systemic toxicity.

PEGylated liposomal doxorubicin formulations are able to extravasate and collect in tumors, and they have been used in the treatment of glioma with some success, increasing progression free survival to 12 months in $15 \%$ of patients ${ }^{115-117}$. The liposomal formulation is necessary since free doxorubicin (DOX) exhibits systemic toxicity and is unable to cross the BBB ${ }^{115}$. However, liposomal DOX is also able to extravasate and collect in other tissues, notably the skin, producing tissue damage and discomfort ${ }^{117}$. FUS-mediated BBBD may facilitate the use of free DOX, generating high intratumor drug concentrations while preventing systemic toxicities associated with the liposomal formulation. While FUS was capable of delivering up to 17-fold increases in DOX concentration in healthy brain tissue, in the GL261 mouse model of GBM, treatment with FUS and free DOX increased DOX concentrations in the tumors by only 4-fold compared to contralateral controls, although this increase was significant. Animals treated with FUS + free DOX had improved survival times $($ IST median $=68 \%$ ) as well, and did not show effects of systemic drug toxicities ${ }^{5}$. While this work indicates that FUS can improve the delivery of free drug across the blood-tumor barrier, it is also apparent that FUS parameters may need to be optimized for tumor biology rather than healthy brain tissue.

Boron neutron capture therapy (BNCT) offers the ability to eradicate tumor cells without damaging healthy tissue, a characteristic which is particularly appealing for brain applications. It relies on the accumulation of a stable boron isotope in the tumor tissue, followed by irradiation with low-energy neutrons. The accumulated boron absorbs the neutrons and releases high energy particles, destroying the tumor cells ${ }^{118}$. BNCT has achieved some success in head-and-neck cancers ${ }^{119}$, as well as GBM 120,121 , but it is believed that FUS-mediated BBBD may improve BNCT efficacy by increasing the concentration of boron in the tumor tissue. Several rodent studies $104,122,123$ have demonstrated that FUS significantly increases the concentration of BPA$\mathrm{f}$, a boron containing drug, in tumor tissue, as well as homogenizing distribution ${ }^{104}$. Nonetheless, it remains to be seen whether this increase correlates to an improvement in treatment efficacy.

Therapeutic antibodies, while currently showing promise in the treatment of numerous cancers, are too large to cross the BBB. Therefore, antibodies which have 
shown success against various cancers are not beneficial for patients with brain metastases ${ }^{124}$, and antibodies designed to treat neurodegenerative diseases require a delivery system ${ }^{125}$. Early work indicated that FUS-mediated BBBD could be used to deliver endogenous IgG antibodies ${ }^{126}$ as well as functionally intact $D(4)$ receptor targeting antibody ${ }^{127}$, opening the door for therapeutic applications. An exciting recent study in the TgCRND8 model of Alzheimer's disease showed that FUS-mediated BBBD increased glial cell activation and the delivery of endogenous IgG and IgM antibodies, which led to a reduction in plaque load ${ }^{83}$. Further work in this model demonstrated the delivery of anti-A $\beta$ across the BBB, which then bound to the plaques and caused a significant $23 \%$ decrease in plaque surface area. Plaque number and size were both decreased in the anti-A $\beta+$ FUS group ${ }^{99}$. An earlier study in the APPswe/PSEN1dE9 Alzheimer's model indicated that FUS-mediated BBBD produces a 3-fold increase in plaque-bound anti-A $\beta$ compared to non-sonicated tissue ${ }^{128}$. These studies suggest the potential use of FUS-mediated antibody delivery for the treatment of neurodegenerative disorders. Indeed, compared to other transcranial delivery methods, FUS is particularly suited for the long term repeated treatments necessitated by the nature of these disorders due to its noninvasive application and highly localized effects.

Trastuzumab (Herceptin), a monoclonal antibody which binds to the Her2 receptor, has shown promise in the treatment of breast cancer ${ }^{124}$, which frequently metastasizes to the brain. In healthy animals, FUS-mediated BBBD significantly increased the delivery of trastuzumab in sonicated tissue with no apparent toxicity ${ }^{129}$. In a rat model of breast cancer brain metastasis, animals receiving FUS + trastuzumab had significantly smaller tumors (4 of 10 tumors resolved completely) with an ISTmedian of $32 \%$ compared to untreated controls. Commonly, patients with brain metastases are omitted from clinical trials, as many therapeutics which work well against the primary tumor do not cross the BBB and have no efficacy against brain metastases. FUSmediated delivery across the BBB can significantly improve drug delivery and efficacy in the brain, and may permit a wider range of treatment options for patients with brain metastases.

Neurotrophic factor administration has been shown to ameliorate a variety of CNS disorders, including schizophrenia ${ }^{130}$, depression ${ }^{131}$, autism ${ }^{132}$, and Parkinson's ${ }^{133}$. However, like most large molecules, neurotrophic factors do not cross the BBB. BDNF, which shows promise as a neuroprotective agent ${ }^{133}$, maintains its bioactivity after FUSmediated delivery across the BBB and generates significant downstream signaling activity ${ }^{134}$. Neurturin (NTN), another factor that has been identified as a potential therapy for neurodegenerative diseases ${ }^{133}$, has also been delivered successfully ${ }^{134}$. FUS-mediated delivery increased NTN bioavailability by 25 -fold compared to direct injection, and activation of signaling downstream of NTN indicated retention of function ${ }^{100}$. Nonetheless, despite the success with BDNF and NTN, glial cell-line derived neurotrophic factor (GDNF) continues to pose problems for FUS-based delivery. One study ${ }^{135}$ demonstrated a significant increase in the delivery of GDNF in FUS-treated regions; however, another was unable to detect GDNF delivery across the BBB due to rapid breakdown in the bloodstream ${ }^{134}$. 
Immunotherapy is especially intriguing for brain tumor applications, because toxicities associated with traditional drugs pose significant problems for healthy brain tissue ${ }^{136,137}$. However, the presence of the BBB confounds most traditional immunotherapeutic approaches. FUS-mediated delivery of immunostimulatory interleukin-12 (IL-12) significantly increased IL-12 deposition in intracranial C6 gliomas, improved tumor growth control and increased survival $(\text { IST median }=43 \%)^{138}$. This effect was attributed to a significant improvement in the cytotoxic T-lymphocyte/regulatory Tcell ratio in the FUS + IL-12 group, presumably due to a combination of increased IL-12 concentration and vascular permeability, which permitted enhanced cytotoxic Tlymphocyte infiltration ${ }^{138}$. With the success of recent immunotherapy trials, we speculate that FUS-mediated immunotherapy delivery may permit the inclusion of patients with brain metastases who would normally be denied treatment and ultimately represent a turning point in how brain metastases are treated.

\section{LIPOSOMES}

Liposomal drug formulations are popular due to their versatility and biocompatibility 139. Their structure, comprised of an aqueous core and a lipid shell, permits the loading of both hydrophilic and hydrophobic drugs ${ }^{140}$, and the formulation of the lipid shell can be easily modulated for PEGylation ${ }^{141}$, thermosensitivity, and/or targeting ${ }^{142}$. Furthermore, both the size and composition of the liposome can be altered to control circulation time and degradation rate ${ }^{143}$. Liposomes are particularly beneficial for packaging highly toxic drugs, since encapsulated drugs are not bioavailable. Conversely, their larger size makes them more difficult to deliver and FUS may trigger release of the drug payload. The liposomal formulation of doxorubicin, a potent anthracycline, was one of the first drug delivery systems used in combination with FUS $3,12,101,103,144,145$. Treat et al demonstrated that a single treatment combining FUS and liposomal DOX delayed tumor growth and improved survival time (IST median $=24 \%$ compared to $16 \%$ for liposomal DOX alone) in a rat gliosarcoma model. Later work by the same group showed that 3 weekly FUS + liposomal DOX treatments drastically improved survival compared to the liposomal DOX-only group (IST median $=100 \%$ and $16 \%$, respectively), with complete tumor resolution in several animals in the FUS + liposomal DOX group ${ }^{12}$. Nonetheless, several animals did suffer from side effects, including skin toxicity, neural loss and intratumoral hemorrhage ${ }^{12}$. To verify that the combination of FUS and liposomal DOX was not causing additional toxicity, a safety study in healthy animals was conducted that demonstrated only minor damage at the focus in animals that received both liposomal DOX and FUS, believed to be due to high local concentrations of DOX deposited by aggressive FUS settings. Of note, the authors also demonstrated that administering liposomal DOX after treatment caused a 32\% decrease in DOX delivery across the BBB, a finding we have substantiated with $60 \mathrm{~nm}$ polymeric NPs (unpublished studies). A study with animals bearing bilateral 9L gliosarcomas indicated that even late stage tumors benefit from FUS-mediated delivery, with treated tumors showing a two-fold increase in DOX concentration compared to unsonicated controls ${ }^{101}$. FUS treatment also significantly increased the delivery of tumor targeted liposomal DOX formulations in an intracranial mouse xenograft model 103,144 , while decreasing some elements of DOX-related toxicity ${ }^{144}$, presumably due to lower levels of drug in circulation post sonication. While it is still unclear whether intact 
liposomes cross the BBB, it is clear that the combination of liposomal encapsulation and FUS-mediated delivery provide excellent therapeutic results, increasing drug concentrations at the target while minimizing systemic toxicities.

\section{DRUG LOADED MICROBUBBLES}

Microbubbles can also be functionalized for use as drug delivery vehicles. Although drug loading is limited to the lipid or protein shell, the relatively large surface area ( $\sim 50$ $\mu \mathrm{m}^{2}$ ) permits conjugation for both targeting and therapeutics ${ }^{146}$. Because of its hydrophobicity, BCNU has been incorporated into the shell of lipid MBs with some success ${ }^{102,147-149}$. Encapsulation of BCNU within the MB's lipid shell permitted simultaneous BBB opening and local drug delivery similar to that seen with unencapsulated drug, with the added benefit of increased tissue retention time at the target ${ }^{149}$. Treatment with BCNU-MBs and FUS showed excellent tumor control 30 days post inoculation and median survival time was increased by $12 \%$ compared to controls in a $\mathrm{C} 6$ glioma model ${ }^{149}$. The addition of VEGF-R2 to the BCNU-MBs provided antiangiogenic targeting capabilities and further improvements in tumor control and animal survival ${ }^{102}$. The same group also developed DOX-loaded MBs conjugated with superparamagnetic iron oxide (SPIO) NPs ${ }^{150}$, which showed a two-fold increase in DOX deposition within a rat glioma compared to a non-sonicated control. FUS treatment followed by magnetic targeting also deposited SPIO NPs released from the MBs within the tumor tissue, permitting MR-based treatment monitoring. While drug-loaded MBs offer the benefit of highly localized delivery, they may also require higher pressure to release the drug and are limited to the circulation time of the MB itself.

$$
\text { POLYMER-BASED DELIVERY SYSTEMS }
$$

Polymer based NP delivery systems offer several advantages over nonencapsulated drugs or viral delivery systems. These include tailorability, ease of manufacture, improved drug-release profiles and protection from degradation or clearance ${ }^{13,151}$. Combined, these properties can reduce drug doses and drugassociated toxicity while improving therapeutic efficacy 9,14,152-155. Polymer NPs can be loaded with a variety of payloads including soluble or non-soluble drugs ${ }^{14,156-158}$, imaging or theranostic agents ${ }^{159-161}$, or nucleic acids ${ }^{151,162-164}$.

It is well known that enhancing therapeutic distribution in the brain parenchyma will improve efficacy ${ }^{165}$. Indeed, while the limitations imposed by the BBB are widely known, the brain parenchyma itself presents a further barrier to delivery in the brain. The brain tissue barrier consists of a dense nanoporous mesh of electrostatically charged macromolecules, including chondroitin sulfate proteoglycans, hyaluronan, and tenascins 166,167 . These charged molecules form a microstructure that hampers diffusion of macromolecules and vectors, including NPs ${ }^{168,169}$ and viruses ${ }^{28}$, via steric or adhesive interactions. In addition, tumors like GBM contain dense and heterogeneous networks of collagen ${ }^{170}$ and high interstitial pressures ${ }^{171}$ that further limit macromolecule diffusion ${ }^{172-175}$. As a result, until recently, it was thought that the upper size limit to diffusion in healthy brain was as small as $64 \mathrm{~nm}^{176}$. However, it has been shown that an extremely dense (>9 PEG/100 $\mathrm{nm}^{2}$ ) coat of the bioinert and neutrally charged polymer polyethylene glycol (PEG) shields NP surface charge and reduces ECM 
interactions in brain tissue, permitting the diffusion of particles up to $114 \mathrm{~nm}$ in size $169,177,178$ and improving circulation time ${ }^{179}$, which leads to enhanced accumulation in tumors through the enhanced permeability and retention effect ${ }^{180,181}$. Dense PEG coatings have demonstrated remarkable improvements in diffusivity and efficacy with multiple types of polymer ${ }^{151,162,164,177}$. For example, highly-PEGylated "brain-penetrating" NPs (BPNs) continue to diffuse up to 24 hours after delivery, leading to a more homogeneous distribution within the parenchyma ${ }^{178}$. In contrast, all sizes of unPEGylated controls were rapidly immobilized within the ECM. Unsurprisingly, drugloaded BPNs are more effective than their un-PEGylated counterparts in limiting tumor growth after intracranial administration. Additionally, BPNs are also an effective vehicle for gene delivery in the brain, and have demonstrated remarkable efficiency after intracranial administration ${ }^{162}$. These BPN represent an important advance in polymeric delivery systems, as evading the BBB is only the first major obstacle to drug and gene delivery in the brain - a point eloquently demonstrated by the lack of success with the Gliadel wafers. Therapeutics must be delivered well beyond the vasculature, and particles that are able to diffuse within the brain parenchyma greatly increase treatment volume and efficacy.

Polymer-based NP delivery systems are well suited for brain therapies after FUS mediated BBBD. Ideal drug delivery systems for applications in the CNS would include (i) ability to homogeneously distribute within the target volume, (ii) sustained drug release and (iii) long circulation times by avoiding rapid clearance. In combination with FUS as a non-invasive strategy to bypass the BBB, polymeric brain-penetrating NPs have potential to overcome many of the hurdles associated with drug and gene delivery in the brain.

\section{ULTRASOUND AND IMMUNOMODULATION}

\section{HISTORY OF CANCER IMMUNOTHERAPY}

William Coley may be regarded as the father of cancer immunotherapy. A surgeon practicing in New York in the 1890's, Coley was intrigued when one of his cancer patients experienced complete remission following two attacks of erysipelas caused by acute infection with the bacteria Streptococcus pyogenes. In an effort to replicate this result, he began injecting his cancer patients with streptococcal cultures. Some patients' tumors regressed, and Dr.Coley published his results in 1892, marking the first attempt at harnessing the body's antitumor immune capacity ${ }^{182}$.

Coley's Toxins, as this protocol became known, achieved a cure rate of $10 \%{ }^{183}$, cancer immunotherapy was generally regarded as infeasible by physicians of the time. The prevailing theory was that cancer cells would be regarded as 'self', and therefore would be ignored by the immune system. With the advent of chemotherapeutics in the 1950's, interest in cancer immunotherapy waned. General opinion was further influenced by several publications that demonstrated that lymphocytes which react to 
'self' are actively deleted ${ }^{184}$, despite evidence that tumor-associated antigens exist 185,186

In the 1960's Burnet published his theory on immunosurveillance, which states that one of lymphocytes' primary functions is to recognize and kill transformed cells before they develop into a tumor ${ }^{187}$. This revitalized view of cancer immunotherapy was discounted once again, however, by work demonstrating the efficiency of deletion of auto-reactive T cells ${ }^{188,189}$. Cancer immunotherapy did not experience a revival until the 1980's.

Beginning in the 1980's, evidence began mounting in favor of cancer immunotherapy as a valid treatment approach. Following the identification of tumorassociated antigens ${ }^{190,191}$ and the demonstration of dendritic-cell mediated activation of a tumor-specific T cell response ${ }^{192}$, the field experienced a significant renaissance ${ }^{193}$. With the recent FDA approval of multiple immune-based cancer therapies, cancer immunotherapy is becoming the new gold standard in the treatment of many types of cancer ${ }^{194-196 .}$

\section{IMMUNE CELLS AND THEIR ROLES IN CANCER IMMUNOLOGY}

Following the discovering of tumor-associated antigens in the 1950's, the primary focus of cancer immunology was $T$ cell response. However, natural killer cells (NK, 197,198) and macrophages ${ }^{199}$ have also been implicated in cancer.

The role of the adaptive immune system in preventing and controlling cancerous cell growth was established based on the identification of several immunogenic tumorassociated antigens ${ }^{190,191}$ and the observation that mice deficient in mature $B$ and $T$ cells experience higher rates of cancer than immunocompetent mice ${ }^{200}$, among other evidence. It has since been shown that activated dendritic cells are able to generate a powerful antitumor T cell response ${ }^{192}$, and that cancer cells evolve in ways that either make them less visible to T cells (e.g. decreased expression of major histocompatibility complexes ${ }^{201}$ ) or protect them from T-cell attack (e.g. increased expression of T-cell inhibitor PD-L1 ${ }^{202}$ ), further bolstering the theory that T cells are heavily involved in cancer progression.

The canonical antitumor immune response is depicted in Figure 4. Tumorassociated antigens activate dendritic cells, which in turn activate cytotoxic $T$ cells (CD8+ T cells). CD8 T cells are able to directly kill cancer cells displaying antigens to which they have been exposed, in addition to releasing cytotoxins ${ }^{203}$. The function of CD4+ helper T cells in cancer immunology is not as prominent, but their importance in antitumor immune response is highlighted by the prognostic use of CD4/CD8 ratios ${ }^{204}$. CD4 cells are also able to recognize and directly kill abnormal cells, but this process is mediated by antigens expressed by the major histocompatibility complex II, which is much rarer than the version expressed on CD8 T cells. However, CD4 T cells provide other functions that may aid the antitumor immune response, including priming CD8 $T$ 
cells, activating antigen presenting cells, and producing excitatory cytokines ${ }^{205}$. On the other hand, regulatory T cells (Tregs) are known to suppress CD8 and CD4 T cell activity during antitumor response, and tumor infiltration by Tregs is a predictor of poor clinical outcome ${ }^{206}$. The CD8/Treg ratio can predict patient response to immunotherapies ${ }^{207}$, emphasizing the importance of the balance of $\mathrm{T}$ cell populations within the tumor microenvironment.

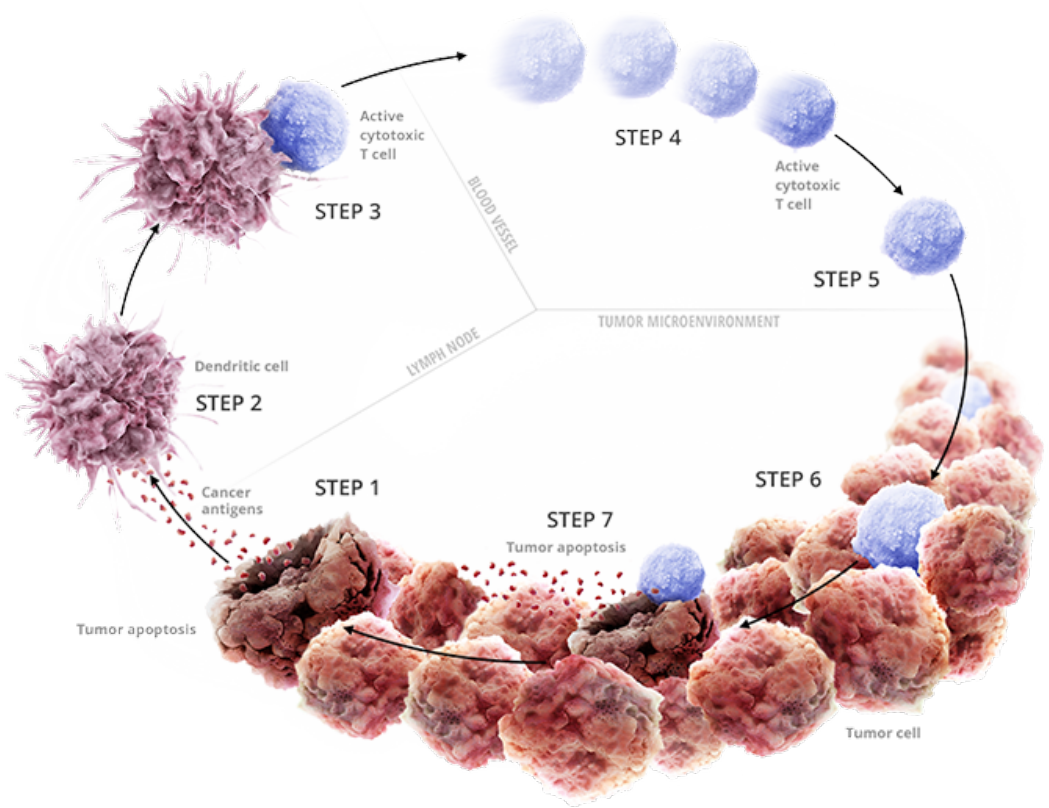

Figure 4: T cell mediated antitumor response www.researchcancerimmunotherapy.com

Other immune cell types have been implicated in antitumor immune responses in recent years. Natural killer cells can recognize tumor cells in the absence of antigen presentation, enabling them to respond immediately. Additionally, they produce several immunostimulating cytokines, most notably TNFa and IFNy, which in turn activate macrophages and dendritic cells, initiating an antigen-based adaptive immune response. ${ }^{198}$ Increased NK infiltration is associated with better prognosis in several types of cancer ${ }^{208}$. In contrast, increased macrophage infiltration correlates with both positive and negative patient outcomes, depending on the type of cancer and the dominant macrophage subset ${ }^{209,210}$. M1 macrophages are pro-inflammatory, and thus considered to be antitumorigenic. M1 polarization is stimulated by INFY, and causes production of high levels of other immunostimulatory cytokines such as TNFa and IL-12, which can in turn induce a T-cell mediated antitumor response. ${ }^{209}$ Alternately, M2 macrophages are tumorigenic, promoting angiogenesis, invasion and metastasis, and immunosuppression ${ }^{209}$. Therapies which can induce an M2 to M1 switch may provide potent tumor control. 
It is now accepted that tumors undergo immunoediting, a process by which cancer cells adapt to evolutionary pressures from the immune system. Over time, this leads to the development of immune privileged tumors, which are able to evade detection and destruction by the immune system. Immunoediting can be divided into three phases: elimination, equilibrium and evasion (Figure 5). ${ }^{193}$

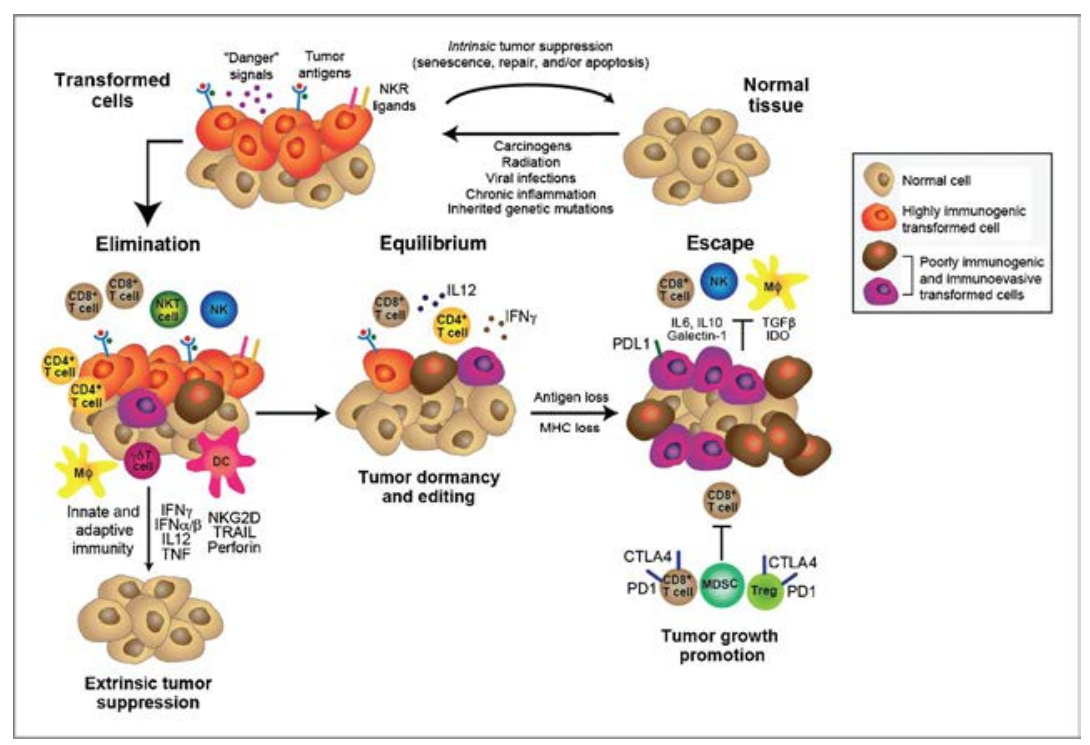

Figure 5: Cancer immunoediting: elimination, equilibrium and escape. ${ }^{213}$

The elimination phase developed from early theories on immunosurveillance ${ }^{193}$. Early in tumor progression, innate immune cells are alerted to the presence of abnormal cells, most likely as a result of cytokine release due to angiogenesis and stromal remodeling occurring in the tumor ${ }^{211}$. Incoming NK cells and/or macrophages respond to the threat by producing IFNY - a critical step in initiating an effective antitumor response ${ }^{212}$. INFy initiates a number of antiproliferative, proapoptotic, and angiostatic process and supports NK cell and macrophage activation ${ }^{200}$. As tumor cells begin to die off or are directly killed via interaction with activated NK cells and/or macrophages, tumor-associated antigens are released, leading to activation of an adaptive immune response ${ }^{212}$. Tumor-specific CD4 and CD8 T cells home to the tumor and efficiently kill immunogenic cancer cells expressing tumor antigens to which they have been exposed 212. This process may end in tumor regression, or it may select for poorly immunogenic cancer cells which do not express tumor antigens, leading to the next stage in cancer immunoediting: equilibrium ${ }^{212}$.

The elimination phase produces a small population of poorly immunogenic cancer cells that must continually resist the efforts of innate and adaptive immune cells, as well as antitumorigenic cytokines like IFNy and IL-12 ${ }^{212}$. As genetic instability within the tumor produces new variants, the pocket of cancerous cells undergoes a gradual selection process that may eventually lead to a population of cells that are able to actively escape immunosurveillance ${ }^{213}$. This selection process may occur over 
decades, producing sub-clinical lesions that can lie dormant for years before developing means of immune escape ${ }^{213}$.

The final stage of immunoediting, escape, produces tumors that are the most difficult to combat. Tumors that have progressed to this stage have developed mechanisms to avoid immune detection, through loss of antigen presentation, and/or immune destruction, through expression of immunosuppressive molecules ${ }^{212}$. Possible mechanisms include upregulation of TGF $\beta$ and IL-10, or recruitment of Tregs and M2 macrophages ${ }^{214}$. Cancer cells may also develop IFNy insensitivity, upregulate expression of immune checkpoint inhibitors like PD-L1, or decrease expression of antigen-presenting complexes ${ }^{214}$. Tumors in this stage often have high levels of Treg and M2 infiltration and low NK, CD4 and CD8 T cell infiltration 205,206,208,215. Additionally, CD8 $\mathrm{T}$ cells are typically poorly activated due to extended exposure to immunosuppressive cells and cytokines, leading to an exhausted T cell phenotype 216,217. Immunotherapies which can reverse $T$ cell exhaustion ${ }^{218}$, promote the infiltration of activated NK ${ }^{198}$, M1 macrophages ${ }^{209}$ or CD8 T cells ${ }^{18}$, or block the effects of immunosuppressive molecules ${ }^{219}$ can reinvigorate the antitumor immune response, leading to effective tumor control and eradication.

\section{CHECKPOINT BLOCKADE IN CANCER IMMUNOTHERAPY}

Although a multitude of immune-based approaches have been suggested for the treatment of cancer, immune checkpoint blockade has gained the most traction. To date, multiple antibody-based therapies targeting either the cytotoxic T-lymphocyte associated pathway (CTLA-4) or the programmed death receptor (PD-1) axis have been FDA-approved for the treatment of a variety of cancers, and are in clinical trials for additional cancer applications ${ }^{219-221 .}$

In normal tissue, CTLA-4 is expressed on T cells in the lymph node, and competes with the stimulatory molecule CD28 to bind their shared ligand, B7, which is expressed on antigen-presenting cells (Figure 6). When bound, CTLA-4 acts as an immune suppressor, reducing $\mathrm{T}$ cell function to mediate central immune tolerance. While the B7 ligand is rarely expressed on tumor cells, tumor resident Tregs often express high levels of CTLA-4. It is theorized that anti-CTLA-4 antibodies block the CTLA-4 receptor on these Tregs, reducing their inhibitory function. ${ }^{17,222,223}$ Two monoclonal antibodies (Ipilimumab and Tremelimumab) against CTLA-4 have been developed are FDA-approved or in clinical trials for several types of cancer, most notably melanoma 222,224 . When used as a monotherapy, responders show significant, durable responses, both in primary and metastatic disease, indicating that a tumorspecific immune response has been activated ${ }^{194,223,224}$. However, the objective response rate varies between $7-15 \%{ }^{224}$. 


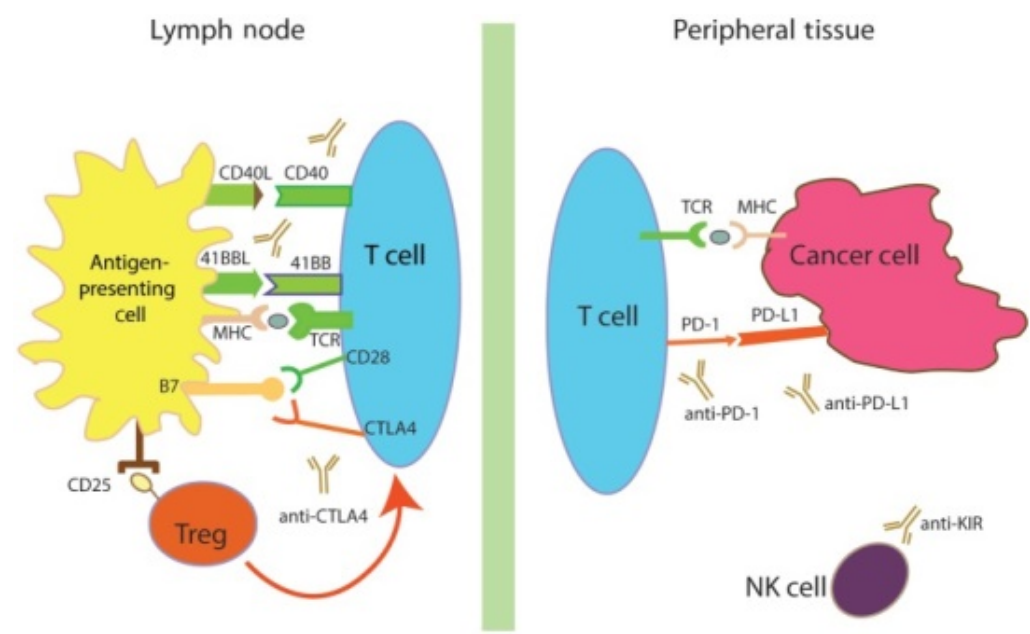

Figure 6: Immune checkpoint blockade in cancer immunotherapy. ${ }^{224}$

The PD-1 receptor and its ligands (PD-L1 and PD-L2) mediate autoimmunity in healthy peripheral tissues ${ }^{224}$. The PD-1 receptor is expressed on CD4 and CD8 T cells, but may also be present on natural killer and dendritic cells, as well as macrophages ${ }^{224}$. PD-L2 is expressed on dendritic cells and macrophages, while PD-L1 is often upregulated in cancerous tissue and is associated with a poor prognosis 202,224-227. When the PD-1 receptor binds either PD-L1 or PD-L2, it reduces the T cell's antitumorigenic functions (Figure 6), including cytokine production, cytotoxic activity and proliferation ${ }^{224}$. Multiple antibodies, targeting either the receptor or the PD-L1 ligand, are in development, and several have already been FDA approved (Nivolumab and Pembrolizumab) for the treatment of several types of cancer 219,222,228-230. As with CTLA-4, responders demonstrate significant increases in survival, and the side effect profile is manageable ${ }^{219,222,228}$. When used as a monotherapy, the patient response rate varies between 20 and $30 \%$, depending on the type of cancer, the drug, and the dose administered ${ }^{224}$.

Given that immune checkpoint monotherapies produce low response rates and generate upregulation of alternate checkpoint inhibitors ${ }^{231}$, there has been considerable interest in combination therapies in recent years. Combining CTLA-4 and PD-1/PD-L1 inhibitors increased response rates to more than $50 \%$, with tumor regressions greater than $80 \%$ in all responders 224,232 . Additional efforts have been made to combine one or both of these checkpoint inhibitors with other immunomodulatory drugs, including granulocyte macrophage colony-stimulating factor, anti-KIR, IL-21, and pegIFN, among others ${ }^{219}$. Preclinically, multiple checkpoint blockade has been successful in curing even highly aggressive cancers such as glioblastoma ${ }^{218,233-238}$. Generally, nonredundant immunomodulation produces significant benefit compared to either therapy alone, but is also associated with more frequent and more significant side effects ${ }^{224}$. Thus, alternate combination therapies with treatments that do not directly modulate immune function have been proposed. Therapies which increase tumor antigen exposure or improve $\mathrm{T}$ cell infiltration are of considerable interest as adjuvant therapies 
for immune checkpoint blockade, especially since the number and fraction of infiltrating CD4, CD8 and Treg cells can predict patient response to immunotherapy 18,207,239.

\section{ULTRASOUND AS AN IMMUNE ADJUVANT}

Ablative techniques can act as potent immune adjuvants. Radiation has been shown to enhance the efficacy of various immunotherapies, most likely through increased antigen exposure and changes in cytokine signaling following radiationinduced tumor cell death 236,240-242. Similarly, high intensity focused ultrasound (HIFU) has been investigated as a potential immune adjuvant.

Thermal ablation via HIFU has been shown to significantly increase the expression of various heat shock proteins (HSP), including HSP27, 60, 70, 72, and 73, both clinically ${ }^{243-245}$ and preclinically ${ }^{246-248}$. HSPs have been shown to induce innate immune responses, as well as enhance tumor antigen presentation ${ }^{249,250}$. HIFU treatment has also been shown to increase the release of immunostimulatory or antitumorigenic molecules such as IL-12 ${ }^{244,246}$, IFNy ${ }^{244}$, and TNFa ${ }^{244,246}$, and decreased the levels of the immunosuppressive molecule IL-10 ${ }^{244}$. Conflicting studies report that HIFU may either increase ${ }^{245}$ or decrease ${ }^{251}$ the release of VEGF and TGF $\beta$, although it should be noted that these studies were done in different cancer types. Given the functions of these molecules, it should not be surprising that HIFU-treated tumors show significant increases in DC and macrophage activation and infiltration 246,252-255, NK cell activity 253,256 , CD4 and CD8 T cell infiltration 18,251,252,256-263 and improvements in CD4/CD8 T cell ratios 18,251,256,260,264. In preclinical models, animals demonstrated resistance to tumor rechallenge following HIFU treatment 251,258,263, indicating that a memory response had been generated against the treated tumors.

Interestingly, sparse-scan ${ }^{254}$ or mechanical ablation $246,258,263$ techniques, which either leave part of the tumor intact or do not thermally denature tumor antigens, showed improvements in activation of antigen presenting cells and increases in CD8 T cell activity when compared to thermal ablation of the entire tumor mass. These observations, in conjunction with tumor rechallenge experiments, provide strong evidence that HIFU-induced antitumor immunity is an antigen-mediated response ${ }^{265}$. This conclusion has sparked conjecture that low-intensity treatments which increase antigen exposure without destroying the tumor (the source of antigen) may elicit a more powerful response.

There is preliminary evidence to suggest this approach may be feasible, but the mechanism is poorly understood. Two studies have demonstrated that low-power FUS can deliver NK cells across the blood-brain barrier 86,266 . Liu et al tested several different low-powered ultrasound protocols in a preclinical model of colon cancer. Pulsed ultrasound in conjunction with microbubbles did not induce a temperature rise in the tumor, but did enhance vascular permeability to dextrans and produced up to $34 \%$ reduction in tumor growth 16 days after US exposure. They showed a significant increase in CD8 T cells within the tumor by day 18, while Treg numbers remained the 
same. Interestingly, CD4 T cell tumor populations increased within 24 hours before returning to control levels by day 3 . The CD8/Treg ratio was significantly improved by day 3 and remained elevated at day 18. ${ }^{267}$ Given the pressures used and the marked enhancement in dextran permeability, it is certain that this protocol produced vascular disruption within the tumor. Hunt et al utilized low-power US in combination with microbubbles in a protocol designed to decreased tumor perfusion, under the assumption that antivascular therapy can induce a hypoxia-mediated immune response. Their protocol produced a $40-70 \%$ decrease in perfusion following treatment, depending on the settings, as measured by contrast-enhanced sonography. 24 hours following treatment, the more aggressive protocol produced significant increases in infiltrating $T$ cells, but no increase infiltrating B cells. Additionally, treated tumors showed increased vessel dilation and expression of the hypoxia marker HIF1a. ${ }^{268}$ Taken together, this body of work suggests three possible mechanisms by which low-intensity ultrasound and microbubble therapy may act as an immune adjuvant, summarized in Table 1.

Table 1: Proposed mechanisms of ultrasound-induced immune response

\begin{tabular}{|c|l|}
\hline Proposed Mechanism & \multicolumn{1}{c|}{ Potential Effects } \\
\hline Endothelial cell activation & $\begin{array}{l}\text { Increased cell adhesion molecule expression } \\
\text { Increased chemokine expression }\end{array}$ \\
\hline Microvascular disruption & $\begin{array}{l}\text { Improved immunotherapeutic delivery } \\
\text { Enhanced trans-endothelial immune cell trafficking } \\
\text { Increased release of antigen into the blood stream }\end{array}$ \\
\hline Tumor stroma disruption & $\begin{array}{l}\text { Improved immune cell trafficking within stroma } \\
\text { Enhanced antigen exposure } \\
\text { Increased chemokine release }\end{array}$ \\
\hline
\end{tabular}


BRAIN-PENETRATING NANOPARTICLES

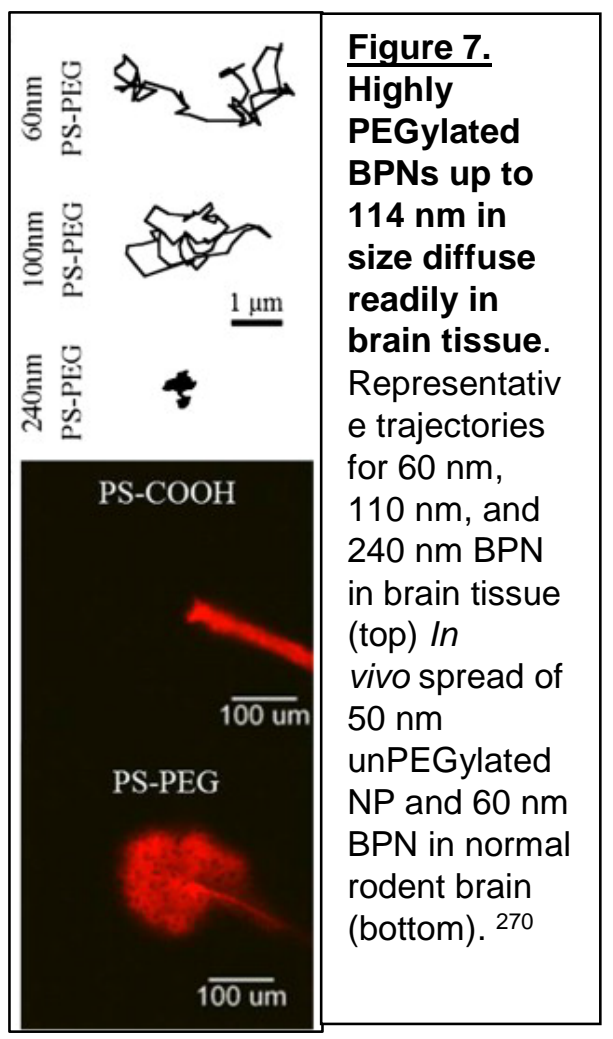

This study is innovative in several ways. We are the first ${ }^{177}$ to demonstrate FUS-mediated delivery of large polymeric nanoparticles across the BBB and BTB or determine the efficacy of this nanoparticle delivery system in an animal model of GBM. Furthermore, we are the first to examine the spatial and compartmental distribution of particles following FUS-mediated delivery across the BBB and BTB. Additionally, the particle system we use is specifically engineered to diffuse within the brain parenchyma. While the limitations imposed by the BBB are widely known, the brain parenchyma itself presents a further barrier to delivery in the brain. It has been shown that an extremely dense (> 9 PEG/100 $\mathrm{nm}^{2}$ ) coat of the bioinert and neutrally charged polymer polyethylene glycol (PEG) shields NP surface charge and reduces ECM interactions in brain tissue, permitting the diffusion of particles up to $114 \mathrm{~nm}$ in size (Fig 7) $169,177,178$ and improving circulation time ${ }^{179}$. When injected directly into an intracranial tumor, the drugloaded, biodegradable highly PEGylated BPNs used in this work are more effective than their un-PEGylated counterparts in limiting tumor growth ${ }^{178}$. Delivery of these particles with FUS-mediated BBB/BTB disruption would eliminate the need for an invasive procedure. These BPNs represent an important advance in polymeric delivery systems, as evading the BBB is only the first major obstacle to drug delivery in the brain - a point eloquently demonstrated by the lack of success with the Gliadel wafers. Therapeutics must be delivered well beyond the vasculature, and particles that are able to diffuse well beyond the cerebral vasculature after FUS-mediated BBBD may greatly increase treatment homogeneity and efficacy.

\section{ULTRASOUND AS A DELIVERY SYSTEM}

While the chemo- and immunotherapeutics used in this project are FDA approved and well characterized when delivered intravenously, the use of ultrasound to enable or enhance their delivery to the target site is innovative. Furthermore, possible interactions between these therapeutics and ultrasound have not been explored. FUSmediated delivery of BPNs is particularly intriguing, since little is known about the interactions between polymeric nanoparticles and FUS, and nothing of this size ( 60 $\mathrm{nm}$ ) has been delivered across the BBB or BTB with FUS and bears characterization. Noninvasive delivery of a systemically administered biodegradable particle would allow 
repeated treatments for patients undergoing chemotherapy, and would also decrease the number of treatments (compared to unencapsulated drug), given the extended drug release profile of these BPNs.

\section{ULTRASOUND AS AN IMMUNE ADJUVANT}

Although the immune response following tumor ablation with HIFU is fairly well characterized 253,254,265, the immune response following low-intensity ultrasound and microbubble therapy is poorly documented and the mechanisms are unknown 267,268. Additionally, no one has yet attempted to determine the relative contributions of the innate and adaptive immune systems, or T cell trafficking versus proliferation, following either high or low-intensity ultrasound. Furthermore, neither modality has been combined with an immunotherapeutic in an attempt to produce synergy. Understanding the mechanisms of ultrasound-mediated antitumor immune responses will enable more educated approaches to combinatorial therapies. 
Designing an MR-guided focused ultrasound protocol for the delivery of $60 \mathrm{~nm}$, brain-penetrating nanoparticles across the blood-brain barrier in the normal rat brain.

Polymeric nanoparticles large enough to carry an efficacious drug load are on the order of $100 \mathrm{~nm}^{14}$, much larger than the small molecule drugs that have been delivered using FUS. Since infiltrating tumor cells are frequently found in areas with normal BBB function ${ }^{10}$, it is important to assess FUS's delivery capability in the healthy brain. MRguided FUS and MBs and will be used to disrupt the BBB in the presence of circulating BPNs. Contrast-enhanced and T2*-weighted MR images, as well as MR thermometry, will be used to assess protocol safety. BPN delivery and distribution will be assessed using confocal microscopy.

Parts of this chapter have been published in Nance E, Timbie K, Miller GW, et al. Noninvasive delivery of stealth, brain-penetrating nanoparticles across the blood-brain barrier using MRI-guided focused ultrasound. J Control Release. 2014;189:123-132.

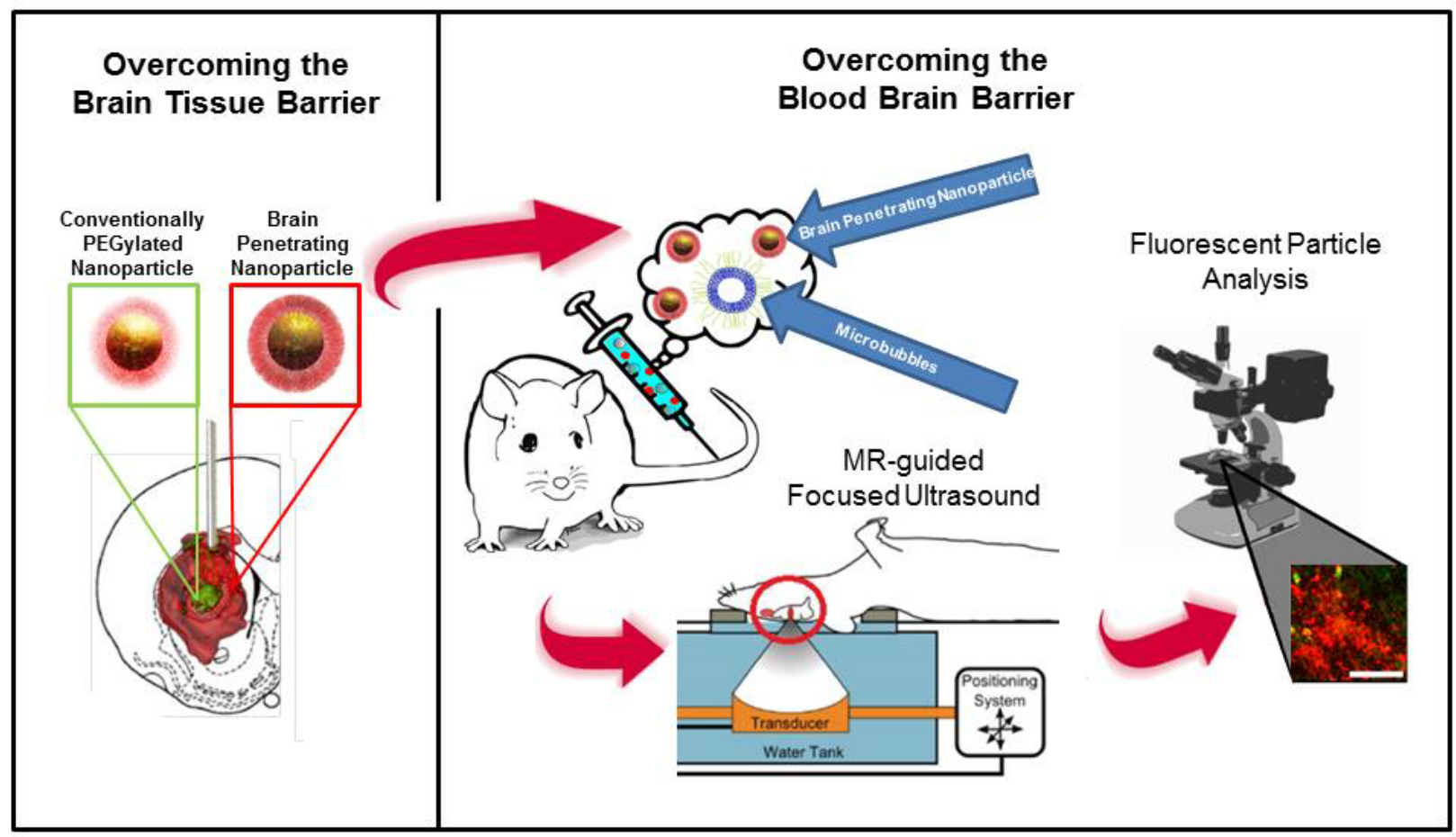


Many therapeutic agents have potential for treatment of central nervous system (CNS) disorders; however, few are able to cross the blood-brain barrier (BBB) and/or penetrate within CNS tissue. The BBB is essential for the maintenance of the CNS environment and regulates the traffic of most molecules to and from the brain [1,2]. Unfortunately, the BBB also limits systemically administered drugs from reaching the brain in therapeutically relevant concentrations [3,4]; thus, drug dose and efficacy are often limited by systemic side effects [5]. In the case of some CNS disorders, such as glioblastoma, Alzheimer's, Parkinson's, cerebral palsy, epilepsy and stroke, the BBB can be impaired and "leaky" [6,7]; however, this impairment is often heterogeneous and diseased cells are often found in normal brain parenchyma in regions supplied by healthy blood vessels with normal BBB function [8]. Other CNS disorders, including lysosomal storage diseases [9], depression [10], and recurrent migraines [11] present even higher obstacles to effective drug delivery into the brain.

Transcranial MRI-guided focused ultrasound (MRgFUS) can non-invasively permeabilize the BBB in a safe, reversible fashion. Image guidance adds the ability to target specific regions with a high degree of accuracy $[12,13]$. Using this strategy, standard microbubble (MB) contrast agents [14] are first injected intravenously. In the region receiving FUS, the MBs are activated, producing a variety of mechanical, chemical and thermal effects [15-18] that lead to a transient disruption of tight junction complexes and induction of active transport of agents into the brain parenchyma $[19,20]$. Small animal studies have demonstrated that the BBB or blood-tumor barrier permeability is increased by MRgFUS [21], improving delivery of therapeutic agents, such as Herceptin and Doxil, as well as treatment outcomes in experimental models of diseases such as glioma and Alzheimer's [22-25]. Additionally, MRgFUS-mediated BBB disruption in non-human primates was shown to be safe and accurate, while producing no significant brain tissue damage or compromised visual function in highly eloquent occipital cortex regions[26]. Once therapeutics cross the BBB, they next encounter a complex microenvironment within the brain extracellular space (ECS), which significantly limits and controls their movement. The ECS is anisotropic with mixed electrostatically charged and hydrophobic regions comprising $15-20 \%$ of the total normal brain volume [27]. Although it was previously thought that a therapeutic nanoparticle must be smaller than $64 \mathrm{~nm}$ to penetrate within the ECS [28], it was recently shown that much larger particles, up to $114 \mathrm{~nm}$ in size, can penetrate within normal brain parenchyma, if densely coated with low molecular weight poly(ethylene glycol) (PEG) [29]. PEG sterically stabilizes nanoparticles and minimizes protein absorption, increasing nanoparticle circulation time. However, PEGylated stealth particles also show decreased interactions with cells, limiting cell uptake or passage across an intact BBB.

The ideal method to deliver drugs to the CNS would include (i) a long circulating nanoparticle that can avoid rapid clearance by the reticuloendothelial system (RES), (ii) a noninvasive approach to bypass the BBB, (iii) the ability of the nanoparticle to 
penetrate within the brain parenchyma and (iv) provide sustained release of a therapeutic agent at the sites of disease. We hypothesized that coupling MRgFUSmediated BBB opening with long-circulating, brain penetrating nanoparticles (BPNs) would achieve significant accumulation and spread of BPNs in select regions of the brain with minimal side-effects. We designed and characterized the behavior of densely PEGylated NPs within brain tissue and demonstrated that $60 \mathrm{~nm}$ BPNs can penetrate the rat brain parenchyma when delivered with MRgFUS. This is the first study to show the successful accumulation of a biodegradable polymeric BPN in the brain in combination with MRgFUS and MBs. This approach represents a promising strategy to overcome the significant hurdles for drug delivery to the brain and improve therapeutic efficacy for many diseases.

EXPERIMENTAL METHODS

\section{STUDY DESIGN}

To test the hypothesis that focused ultrasound can safely enhance the delivery of large polymeric nanoparticles across the BBB, female $160 \mathrm{~g}-180 \mathrm{~g}$ Sprague-Dawley rats were sonicated with peak negative pressures ranging from 0.4 $\mathrm{MPa}$ to $2.0 \mathrm{MPa}$. MR imaging was used to evaluate safety and verify BBB disruption, and confocal imaging of fluorescently labelled PEGylated BPNs developed by the Hanes lab at Johns Hopkins University was used to evaluate efficacy. Both PEGylated polystyrene and biodegradable PGLA BPNs were delivered intravenously at a dose of $15 \mu \mathrm{g} / \mathrm{g}$ body weight immediately prior to sonication. Animals were sonicated with either 4 or 8 spots as described in the following sections, and were euthanized either 1 or 24 hours after treatment. All animal experiments were carried out at the University of Virginia following National Institutes of Health guidelines and local Institutional Animal Care and Use Committee regulations.

\section{MICROBUBBLE FABRICATION AND CHARACTERIZATION}

The albumin MBs used in this study are similar in composition, size and distribution to Optison (GE Healthcare). MBs were produced by sonicating $(20 \mathrm{KHz}, 30$ s) a $1 \%$ solution of serum albumin in normal saline with an ultrasound disintegrator (XL2020, Misonix, Farmingdale, NY) equipped with an extended $1 / 2$ " titanium probe. The headspace in the flask containing the solution was filled with octafluoropropane gas. MBs were sized and counted using a Coulter Counter (Multisizer 3, Beckman Coulter, Fullerton, CA) with an orifice size of $50 \mu \mathrm{m}$ and a lower measurement limit of $1 \mu \mathrm{m}$.

\section{PS-PEG NANOPARTICLE PREPARATION AND CHARACTERIZATION}

The following work was performed at Johns Hopkins University. 
Model nanoparticles were prepared as previously described ${ }^{169}$. Briefly, 40- to 200 $\mathrm{nm}$ red fluorescent $\mathrm{COOH}$-modified polystyrene (PS) particles (Molecular Probes) were covalently modified with methoxy (MeO)-PEG-amine $\left(\mathrm{NH}_{2}\right)$ (5 kDa MW; Creative PEG Works) by carboxyl amine reaction. An excess of MeO-PEG-NH 2 was added to the PS particle suspension and mixed to dissolve the PEG. $N$-Hydroxysulfosuccinimide (Sigma) was added to the reaction tube and $200 \mathrm{mM}$ borate buffer, $\mathrm{pH}$ 8.2, was added to a 4-fold dilution of the starting volume. 1-Ethyl-3-(3-dimethylaminopropyl) carbodiimide (EDC, Invitrogen) was added and particle suspensions were placed on a rotary incubator for 4 hours at $25^{\circ} \mathrm{C}$, then centifuged (Amicon Ultra $0.5 \mathrm{~mL} 100 \mathrm{k}$ MWCO; Millipore). Particles were resuspended in ultrapure water to the initial particle volume $(100 \mu \mathrm{L})$ and stored at $4^{\circ} \mathrm{C}$ until use. Physicochemical characteristics of NP were determined using a Zetasizer NanoZS (Malvern Instruments, Southborough, MA). All particles were diluted in $10 \mathrm{mM}$ $\mathrm{NaCl}$ (diluted from phosphate buffered saline) and dynamic light scattering (DLS) was employed to determine the hydrodynamic diameter and polydispersity index (PDI) at a backscattering angle of $173^{\circ}$. The $\zeta$-potential, a measure of particle surface charge, was determined using laser Doppler anemometry. The size and morphology of NP was determined using a Hitachi H7600 transmission electron microscope (TEM, Hitachi, Japan).

\section{POLYMER LABELING WITH A FLUORESCENT DYE}

The following work was performed at Johns Hopkins University.

For fluorescent labelling of biodegradable BPNs, PLGA (75:25) polymer (MW: 15kDa; Jinan Daigang Biomaterials Co. Ltd., Jinan, China) and PEG-PLGA (75:25) (25\% PEG) copolymers (Total MW: 20 kDa; Jinan Daigang Biomaterials Co. Ltd., Jinan, China) were labeled with AlexaFluor 555 cadaverine (AF 555, Molecular Probes) or AlexaFluor 647 cadaverine (AF647, Molecular Probes) fluorescence dye, as described previously ${ }^{269}$. Briefly, the polymers were first dissolved in dichloromethane (DCM) and were activated by $\mathrm{p}$-nitrophenyl chloroformate and pyridine. The reaction was carried out for 3 hours under constant stirring. The activated polymers were precipitated in $4^{\circ} \mathrm{C}$ ethylene ether, washed in dimethylformamide (DMF) and were dried overnight under vacuum. Then, the activated polymers and the AF 555 dye were dissolved DMF, and triethylamine was added immediately to the polymer-dye mixture. The reaction was carried out for 4 hours under constant stirring. The fluorescently labeled polymers were precipitated in $4^{\circ} \mathrm{C}$ ethylene ether, washed in DMF, and dried overnight under vacuum. Polymers were stored at $-20^{\circ} \mathrm{C}$ in the dark until use.

\section{PLGA-PEG NANOPARTICLE PREPARATION AND CHARACTERIZATION}

The following work was performed at Johns Hopkins University.

Fluorescently labeled PEG5k-PLGA15k polymers were dissolved in $1 \mathrm{~mL}$ DCM at $20 \mathrm{mg} / \mathrm{mL}$. The polymer solution was added to $5 \mathrm{~mL}$ cholic acid $(0.5 \% \mathrm{CHA})$ 
immediately followed by sonication (30\% magnitude for $2 \mathrm{~min}$ ) in an ice water bath. Then, the emulsified solution was added to $25 \mathrm{~mL}$ of surfactant solutions and stirred for three hours to remove solvent. The sample solutions were filtered and collected. PEGPLGA nanoparticles made in $0.5 \%$ CHA were collected by using centrifugal filtering units (MWCO: $100 \mathrm{kDa}$, Millipore Ltd. Ireland) at $3600 \mathrm{xg}$ for $12-16 \mathrm{~min}$ so that the final sample volume in the filter unit was less than $1 \mathrm{~mL}$. The nanoparticles were washed three times in ultrapure (UP) water. Particles were characterized as described for PSPEG BPNs.

\section{NANOPARTICLE DIFFUSION IN EX VIVO AND IN VIVO RODENT BRAIN}

The following work was performed at Johns Hopkins University.

Rat brain tissue slices were prepared as described previously ${ }^{169} .0 .5 \mu \mathrm{L}$ of fluorescently labeled nanoparticles were injected into the gray matter region using a 10 $\mu \mathrm{L}$ syringe (Hamilton Company, Reno, NV).

For in vivo spread, the cranial window technique was used as previously described ${ }^{169}$. Briefly, a warm agarose solution $(20 \% \mathrm{w} / \mathrm{v})$ was placed over the exposed brain region and a 5-mm glass coverslip was quickly placed prior to agarose cooling and gelatinization to create a stable window. A custom circular metal bar was secured to the adjacent bone and cement (HyBond, Inc.) was then applied to secure the agarose, glass, and metal bar construct rigidly to the calvarium. A channel representing approximately 90 degrees of the cover glass circle was not cemented and left exposed for the glass pipette to insert into the brain. The cranial bar was secured to a custom microscope stage allowing stable imaging of the anesthetized mouse. An upright confocal microscope (Zeiss, Inc.) with a two-photon laser source (Coherent Inc.) tuned to $910 \mathrm{~nm}$ was used for imaging through a $20 \times$ microscope objective [Zeiss Inc., PlanApochromat (NA, 1.0; working distance, $1.9 \mathrm{~mm}$ )]. Images were collected using a nondescanned detector. The micro-injection apparatus attached to a stereotactic manipulator (Drummond Scientific Inc.) was fixed with a glass micropipette (tip diameter $\sim 30 \mu \mathrm{m}$ ), loaded with NP solution, and positioned for injection through the agarose channel into the brain. A blood vessel-free region of cortex was identified, and the micro-pipette was inserted to a depth of 100-200 $\mu \mathrm{m}$ below the pial surface and withdrawn slightly to create a small pocket to receive the injection. The nano-injection device was set to inject $9.2 \mathrm{~nL}$ of particle solution at a rate of $23 \mathrm{~nL} / \mathrm{s}$.

\section{MULTIPLE PARTICLE TRACKING IN RAT BRAIN SLICES}

The following work was performed at Johns Hopkins University.

The transport rate of particles was determined by analyzing the trajectories of the particles as described previously ${ }^{169}$. The particle trajectories in brain tissue slices were recorded by a silicon-intensified target camera (VE-1000, Dage-MTI, Michigan, IN) mounted on an inverted epifluorescence microscope equipped with a 100x oilimmersion objective (N.A., 1.3). Rat brain tissue specimens $(\mathrm{N}=3)$ were used for each 
particle type. The average pore size of the brain ECS and pore size range was estimated based on fitting an obstruction scaling model to the measured particle diffusion rates using maximum likelihood estimation ${ }^{176}$. The model is valid in cases where there is limited interaction between the particles and the ECS components and where fluid in the ECS exhibit micro-viscosity similar to that of water.

\section{HISTOPATHOLOGICAL ANALYSIS OF RAT BRAIN SLICES}

The following work was performed at Johns Hopkins University.

The rat brain tissue slices were studied using standard hematoxylin and eosin (H\&E) staining to identify any changes in histological architecture and cell morphology introduced by the preparation and incubation process. Representative tissue slices $(n=$ 3 ) were preserved in formalin immediately following sectioning in the laboratory and after completing data acquisition, approximately 3 hours following removal, preparation, incubation, and particle imaging. The tissue was removed from the formalin after 24 hours and placed in $70 \%$ ethanol solution until paraffin embedding, sectioning, and H\&E staining. The tissue sections were examined by a board-certified neuropathologist (C.E.) for evidence of tissue changes or damage.

\section{CIRCULATION STUDIES}

The following work was performed at Johns Hopkins University.

Female CF-1 mice (Harlan), 6-8 weeks old, were placed under a heat lamp for several minutes until blood vessels in the tail were dilated. The mice were then placed upright in a mouse tailveiner restrainer (Braintree Scientific), by pulling the mouse by the tail through the slotted tube. The mouse was then restrained by a plug and the tail was swabbed with a $100 \%$ ethanol swab. $100 \mu$ of near-infrared particle suspension in PBS was injected into the tail vein using a $0.5 \mathrm{ml}$ insulin syringe. Mice were imaged at 6 hour, 12 hour, 16 hour, 24 hour, and every day up to one week using the Xenogen IVIS Spectrum optical imaging device and Living Image software (both from Caliperls, Hopkinton, MA). The same imaging parameters were used at all timepoints for all images, with a 5 second exposure and 8 binning.

\section{BLOOD-BRAIN BARRIER OPENING WITH MRGFUS}

All FUS-related animal experiments were carried out at the University of Virginia following National Institutes of Health guidelines and local Institutional Animal Care and Use Committee regulations. On the day of treatment, female Sprague-Dawley rats (180$220 \mathrm{~g}$ ) were anesthetized with an intraperitoneal injection of Ketamine $(40 \mathrm{mg} / \mathrm{kg}$, Fort Dodge) and Dexdomitor ( $0.2 \mathrm{mg} / \mathrm{kg}$, Pfizer) in sterilized water. A tail vein catheter was inserted to allow intravenous (i.v.) delivery of microbubbles, nanoparticles and contrast agent. Animal skulls were depilated and positioned in a degassed water bath coupled to the FUS system. For feasibility studies, rats received an i.v. injection of polydisperse MBs (1E5 MBs/g body weight) immediately prior to sonication. For nanoparticle delivery 
studies, rats received a coinjection of nanoparticles ( $15 \mu \mathrm{g} / \mathrm{g}$ body weight) of MBs (1E5 $\mathrm{MBs} / \mathrm{g}$ body weight) immediately prior to sonication.

All sonications were performed using a $1.14 \mathrm{MHz}$ single element focused transducer (FUS Instruments, Toronto, CA) operating at a $0.5 \%$ duty cycle for 2 minutes. Peak negative pressure was either $0.4 \mathrm{MPa}$ or $0.6 \mathrm{MPa}$ for NP delivery experiments, and ranged from 0.4 to $2.0 \mathrm{MPa}$ for safety studies. Prior to placing the animal on the MRI table, the FUS system was passively aligned with a 3T MRI system (Trio, Siemens) using an anechoic Zerdine phantom (CIRS, Inc, Norfolk, VA). After the animal was coupled to the FUS system, high resolution T1-weighted prescans were performed and utilized to plan sonication focal points with high accuracy. For nanoparticle delivery studies, four locations were sonicated in each animal, spaced $3 \mathrm{~mm}$ apart coronally, $2 \mathrm{~mm}$ to either the left or right of the interhemispheric fissure, and $5 \mathrm{~mm}$ from the top of the skull. Care was taken to remain within the cerebral hemispheres, avoiding the cerebellum and ventricles. All four locations were sonicated within one 2 minute sonication period using interleaved sonication mode. For safety studies, some animals received an additional sonication of four targets on the contralateral side of the brain. Immediately following sonication, MRI contrast agent (0.5 $\mu \mathrm{l} / \mathrm{g}$ body weight, Magnevist, Bayer Health Care, PA) was delivered intravenously and T1-weighted contrast enhanced images were captured to verify BBB disruption. Animals were then removed from the MRI table and placed on a heating pad at $37^{\circ} \mathrm{C}$ for one hour to allow increased nanoparticle uptake and diffusion within the brain parenchyma. $2 \%$ heparinized saline $(0.5 \mathrm{~mL})$ was delivered intravenously 30 minutes post sonication and again $(1.0 \mathrm{~mL})$ one hour post sonication. Animals were euthanized with pentobarbital $(0.5 \mu \mathrm{l} / \mathrm{g}$ body weight) either 1 hour or 24 hours after the sonication.

\section{MR THERMOMETRY}

MRI thermometry was performed in two rats not receiving NP injections, as the movement of the transducer necessary to perform interleaved sonication interfered with image capture. Each rat was sonicated eight times, four locations at $0.6 \mathrm{MPa}$ in the left hemisphere and four locations at $0.4 \mathrm{MPa}$ in the right hemisphere. Sonications were performed individually using the protocol previously described. MBs were reinjected immediately prior to each sonication and six minutes elapsed between each sonication to ensure that bubbles from the previous sonication had been fully cleared.

Thermometry scans were performed every 5 seconds beginning 10 seconds before sonication and ending 20 seconds after completion of the sonication. Rats received MRI contrast agent ( $0.5 \mu \mathrm{l} / \mathrm{g}$ body weight, Magnevist ${ }^{\circ}$, Bayer Health Care, PA) after all eight sonications had been completed. High resolution T1-weighted scans were captured before initiating sonication and immediately following Magnevist ${ }^{\circledR}$ injection.

MR T2* IMAGING

T2* images were captured from twelve rats not receiving NP injections. For all animals, high-resolution T1- and T2*-weighted images were captured before and after sonication. Up to eight locations were sonicated in each animal, no more than four in 
each hemisphere as described for the NP delivery group. Two separate sonications were performed, one per hemisphere, using interleaved sonication mode. Pressures ranged from 2.0 to $0.4 \mathrm{MPa}$. Sonications were spaced six minutes apart to allow clearance of the previous dose of MBs. MRI contrast agent $(0.5 \mu \mathrm{l} / \mathrm{g}$ body weight, Magnevist ${ }^{\circledR}$, Bayer Health Care, PA) was delivered intravenously after sonication and T2*-weighted imaging, followed by high resolution T1-weighted scans to verify BBB disruption.

\section{MRI IMAGE PROCESSING}

T1-weighted contrast enhanced images: T1-weighted post-sonication images were analyzed in MATLAB (Mathworks, Natick, MA) using a code developed in our lab. Contrast enhanced regions were identified using a threshold defined as $45 \%$ of the maximum contrast in the image. Control ROls in the non-sonicated hemisphere were subtracted from the enhanced ROIs to determine the FUS-induced intensity enhancement. ROI size and average intensity were recorded and analyzed using a student's t-test.

Thermometry: MRI thermometry data were analyzed in MATLAB using a code developed by Dr. Miller (Radiology, UVA, Charlottesville, VA). A baseline phase image was reconstructed from an average of 3 pre-sonication scans. All phase images captured during sonication were averaged and the baseline phase image was subtracted. This average phase change image was converted to temperature change and overlaid on high resolution T1-weighted contrast enhanced post-sonication images. Significance was determined using a student's t-test.

\section{PROCESSING AND IMAGING OF SONICATED BRAIN TISSUE}

Following euthanasia, animals' left and right carotid arteries were cannulated. Each artery was perfused with $22 \mathrm{~mL} 2 \%$ heparinized saline prior to brain excision. Brains were dessicated in a $30 \%$ sucrose solution at $4{ }^{\circ} \mathrm{C}$ for 24 hours or until brains sunk. Dessicated brains were placed in O.C.T. compound (Sakura Finetek, Torrance, $\mathrm{CA}$ ) for one hour at $21^{\circ} \mathrm{C}$ before freezing at $-80^{\circ} \mathrm{C}$. Transverse $8 \mu \mathrm{m}$ cryosections were mounted, stained with a 1:200 Lectin 488 (Invitrogen, Grand Island, NY) solution for 1.5 hours at $21^{\circ} \mathrm{C}$ and sealed with Prolong Gold (Invitrogen, Grand Island, NY) for imaging. Rat brain sections were imaged on a Nikon TE300 confocal microscope equipped with a 20x oil objective. An Optronics QuantiFIREXI camera was used to capture the images for analysis.

Confocal images were analyzed in MATLAB using a code developed in our lab. The nanoparticle (red channel) and endothelial (green channel) signals were separated for processing. Greyscale intensity images were morphologically opened and converted to black and white to remove background signal. The original grayscale image was masked using this black and white image, followed by local Otsu thresholding to eliminate overexposure. Finally, morphological closing was used to fill out object borders. The processed nanoparticle and endothelial cell images were subtracted to 
separate nanoparticle signal originating within blood vessels from interstitial nanoparticle signal. Nanoparticle "cloud" area, defined as thresholded NP objects outside of blood vessels, was calculated from this subtracted image as well. In addition, the percentage of vessels containing NPs or in close proximity to a NP cloud ("NP' Vessels") was counted from the processed confocal images. Significance was determined using a student's t-test.

RESULTS

\section{TRANSPORT OF BPN IN NORMAL RAT BRAIN ECS}

To ensure large, therapeutically relevant nanoparticles could penetrate within the rodent brain, we first determined the effect of particle size and surface chemistry on transport rates of modified particles in rat brain tissue. The hydrodynamic diameters of the particles are listed in Table 1.1. The 100-nm and $200-\mathrm{nm} \mathrm{COOH}$-modified particles displayed low transport rates in freshly excised viable rat brain slices, as measured by arithmetic ensemble mean squared displacements ( $<\mathrm{MSD}>)$ (Fig1.1A). At a time scale of 1s, 50-nm, 100-nm, and 200-nm PS-COOH particles were 20,000-80,000-fold slower in an unobstructed physiological relevant medium, artificial cerebral spinal fluid (ACSF), than the equivalent PS-COOH particles.

\begin{tabular}{|l|l|l|l|l|}
\hline $\begin{array}{l}\text { Commercially } \\
\text { Supplied Size } \\
(\mathrm{nm})\end{array}$ & $\begin{array}{l}\text { Surface } \\
\text { Modification }\end{array}$ & $\begin{array}{l}\text { Mean Diameter } \\
\pm \text { SEM }(\mathrm{nm})\end{array}$ & $\begin{array}{l}\zeta \text {-potential } \pm \\
\text { SEM }(\mathrm{mV})\end{array}$ & $\mathrm{D}_{\text {ACSF }} / \mathrm{D}_{\mathrm{b}}$ \\
\hline 40 & COOH & $54 \pm 0.4$ & $-36 \pm 2$ & 76,000 \\
\hline 40 & PEG5k & $61 \pm 6$ & $-0.7 \pm 3$ & 9 \\
\hline 100 & COOH & $95 \pm 2$ & $-42 \pm 2$ & 16,000 \\
\hline 100 & PEG5k & $108 \pm 3$ & $-2.9 \pm 1$ & 60 \\
\hline 200 & COOH & $205 \pm 1$ & $-40 \pm 2$ & 48,000 \\
\hline 200 & PEG5k & $242 \pm 6$ & $-5.4 \pm 1$ & 2,600 \\
\hline
\end{tabular}

Table 1.1: Physicochemical properties of polystyrene nanoparticles and their diffusivity in normal rat brain tissue compared to in ACSF. Effective diffusivity of NPs in normal brain tissue (Db) was calculated at a time scale of $1 \mathrm{~s}$. NP diffusivity in ACSF (DACSF) was calculated with Stokes-Einstein equation and mean particle diameter. Size was provided by the manufacturer, and actual diameter in ACSF at pH 7.0 was measured with dynamic light scattering. $\zeta$-potential and PDI were measured in ACSF at pH 7.0. Size, $\zeta$-potential, and PDI were all measured after 24-hour incubation in ACSF.

We have previously demonstrated we can achieve a dense coating of PEG, 0.9 PEG per nm², on 50-, 100-, and 200-nm PS particles ${ }^{169}$. In this study, the extent of PEG attachment was comparable for all particle sizes as shown by their near-neutral surface charges (Table 1.1). PEGylation greatly increased particle transport rates for 60- and 110-nm particles (PS-PEG). 60-nm and 110-nm PS-PEG particles showed only 9-fold and 60 -fold slower diffusion ( $\mathrm{T}=1 \mathrm{~s}$ ) in brain tissue, respectively, compared with corresponding particles of the same size in ACSF (Fig1.1A,B). The 240-nm PS-PEG particles showed a smaller increase in particle transport compared to uncoated particles of the same size, likely due to significant steric obstruction within the brain ECS, supported by representative particle trajectories (Fig1.1A). 

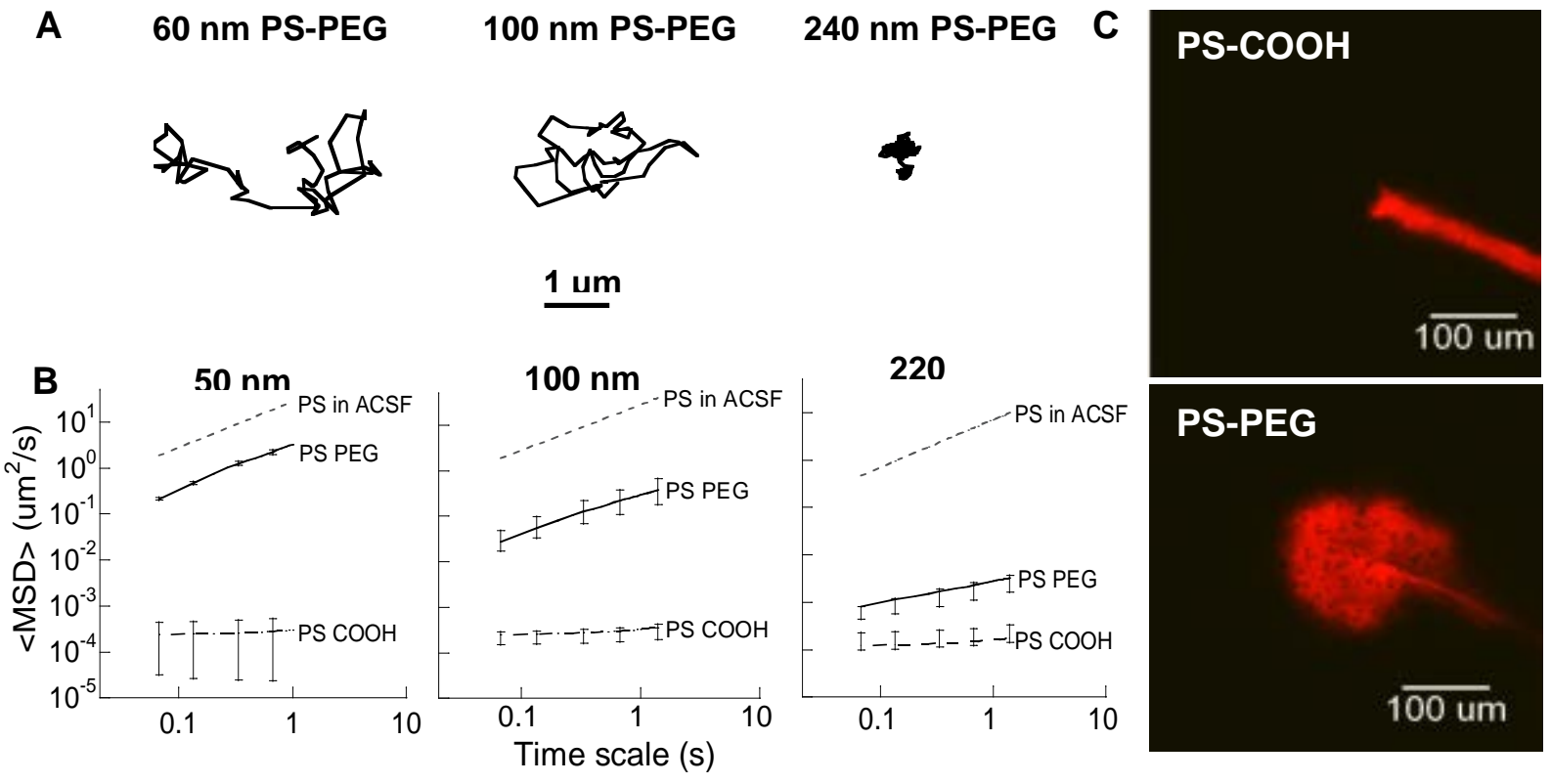

220

D
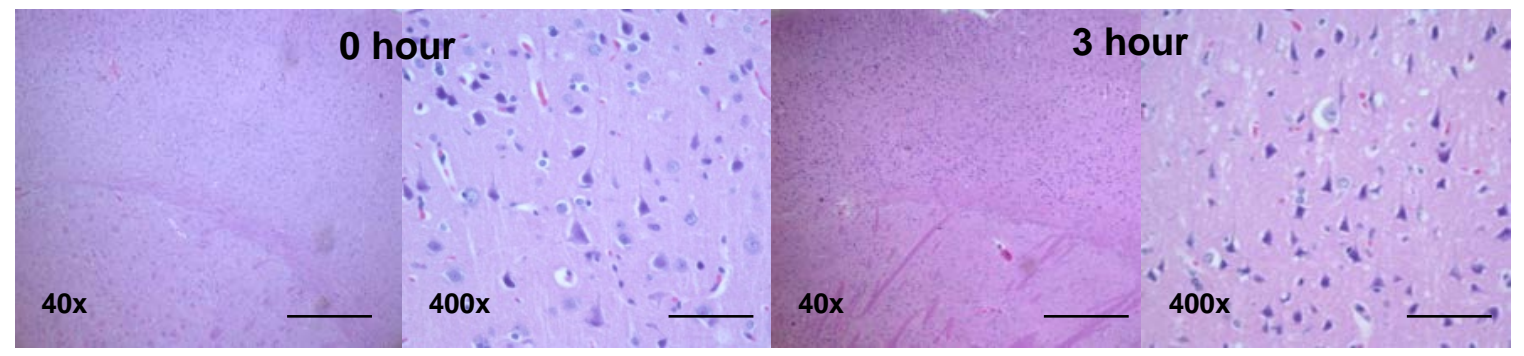

Figure 1.1: Transport rates of various sized nanoparticles in normal rat brain neocortical slices.

(A) Ensemble-averaged geometric mean square displacements ( $\angle M S D\rangle)$ as a function of time scale for $50 \mathrm{~nm}, 100 \mathrm{~nm}$, and $200 \mathrm{~nm}$ PS-PEG and PS-COOH. Data represent the ensemble average of at least three independent experiments, with $n \geq 100$ particles for each experiment. For each experiment, the transport rates of all three particle sizes with two different surface properties were measured in the same brain tissue. Dotted lines indicate theoretical MSD values for the same size PS particles in ACSF. (B) Representative trajectories for $60 \mathrm{~nm}, 110 \mathrm{~nm}$, and $240 \mathrm{~nm}$ PS-PEG in brain tissue exhibiting an MSD equal to the ensemble average at a time scale of $1 \mathrm{~s}$. (C) In vivo spread of $50 \mathrm{~nm}$ PS COOH and $60 \mathrm{~nm}$ PS PEG5k in normal rodent brain. (D) Histology of brain tissue used for ex vivo tissue slices for MPT analysis at 0 hour timepoint immediately following brain removal and sectioning, and at 3 hour timepoint representative of tissue appearance at the time of data acquisition and particle tracking. Scale bar: 40 $\mu \mathrm{m}$.

To further evaluate the extent of impediment to particle transport, the Tdependent, mean-squared displacement $\langle$ MSD $>$ was fitted to the equation $\langle$ MSD $>=$ 4Doтa, where Do is the T-dependent diffusivity and $\alpha$ is the anomalous diffusion exponent that reflects the extent of impediment. $\alpha=1$ represents unobstructed Brownian diffusion, such as particles in ACSF; $\alpha$ becomes smaller as obstruction to particle diffusion increases. The $\alpha$ value for $240-n m$ PS-PEG particles was 0.48 , compared to an a value of 0.78 for $110 \mathrm{~nm}$ PS-PEG particles. The a value for $\mathrm{COOH}-$ modified particles for both 100- and 200-nm PS particles were 0.20 and 0.22 , respectively. As expected from the high transport rates of 60-nm PS-PEG particles, the 
$\alpha$ value was 0.92 compared to an $\alpha$ value of 0.18 for $50 \mathrm{~nm}$ PS-COOH particles, further confirming unhindered diffusion of 60-nm PEG-coated particles in brain ECS, slightly hindered diffusion of $110 \mathrm{~nm}$ well coated particles, and hindered diffusion of 240-nm PEG-coated particles.

Using live-animal imaging, we directly observed 60-nm NP penetration in vivo in mouse brains to confirm findings obtained using ex vivo tissue slices (Fig1.1C). Redfluorescent, $\mathrm{COOH}$-coated NPs and PEG-coated NPs were injected $200 \mu \mathrm{m}$ deep into the mouse cerebral cortex in two regions free of blood vessels, and imaged over a 30 minute time frame. Real-time video microscopy showed that the $\mathrm{COOH}$-coated NPs were immobilized within the tissue, whereas PEG-coated NPs penetrated up to $150 \mu \mathrm{m}$ into the tissue within 30 minutes.

\section{EX VIVO TISSUE INTEGRITY DURING MULTIPLE PARTICLE} TRACKING

As confirmed with previous ex vivo brain slice models ${ }^{169}$, representative hematoxylin and eosin (H\&E)-stained sections indicated no differences between immediate tissue removal (30 minutes) and 3 hours after tissue removal, processing, particle administration, and imaging (Fig1.1D). The lack of cellular swelling, pyknotic nuclei, and ischemia suggests minimal tissue damage was introduced by tissue processing and particle tracking, confirming that the tissue maintains intact cytoarchitecture and functioning tissue physiology within the experimental time frame.

\section{CHARACTERIZATION OF EFFECTIVE PORE SIZES IN THE BRAIN ECS}

To ensure that the observed rapid transport of PEGylated nanoparticles was not biased by a small fraction of fast moving outlier particles, we evaluated the heterogeneity in particle transport rates by examining the distribution of individual particle diffusivities at $1 \mathrm{~s}$ (Fig1.2A). The fastest 75 percent of 60-nm and fastest 65 percent of 110-nm PS-PEG particles exhibited uniformly rapid transport, compared to the fastest 15 percent of 240-nm PS-PEG particles. Fast moving outlier nanoparticles are a subpopulation of interest, as they are more likely to penetrate brain tissue and reach greater distances from the site of entry into the brain. 60-nm PS-PEG particles showed homogenous distribution, with less than an order of magnitude difference between the fastest and slowest group of particles. The fastest 10th percent of 110-nm and 240-nm PS-PEG particles showed 5 and 3 orders of magnitude difference, respectively, from the slowest particles, suggesting that some of the spacing in the ECS could be larger than $100 \mathrm{~nm}$. However, for all other subgroups (i.e. the slowest $90 \%$ of particles) 240-nm PS-PEG particles displayed 2 orders of magnitude lower Deff compared to the same subgroups of 60 - and 110- nm PS-PEG particles, leading to an average Deff representative of hindered particle transport.

An effective pore size of the brain ECS could be determined by fitting the measured diffusion rates of 60-, 110-, and 240- nm PS-PEG particles with an 
obstruction scaling model ${ }^{176}$. Based on the determination that transport was limited due to steric obstruction for larger particles and not due to particle interaction with brain ECS components, diffusion rates for 240-nm PEG-PS particles were incorporated into this model. Using maximum likelihood estimation, the average pore size of normal rat brain ECS was estimated to be between 60-120 nm (Fig1.2B). More specifically, we calculated the pore size distribution to range from $20 \mathrm{~nm}$ to $230 \mathrm{~nm}$. The largest $20 \%$ of pore sizes experienced by particles were $>100 \mathrm{~nm}$ and $14 \%$ of pore sizes experienced by particles were $>200 \mathrm{~nm}$ (Fig1.2C). Overall approximately $60 \%$ of the pores sampled by probe particles were larger than $50 \mathrm{~nm}$. The pore size modeling further corroborates the transport rates observed with multiple particle tracking, and correlates well with data previously published in normal human brain tissue ${ }^{169}$.
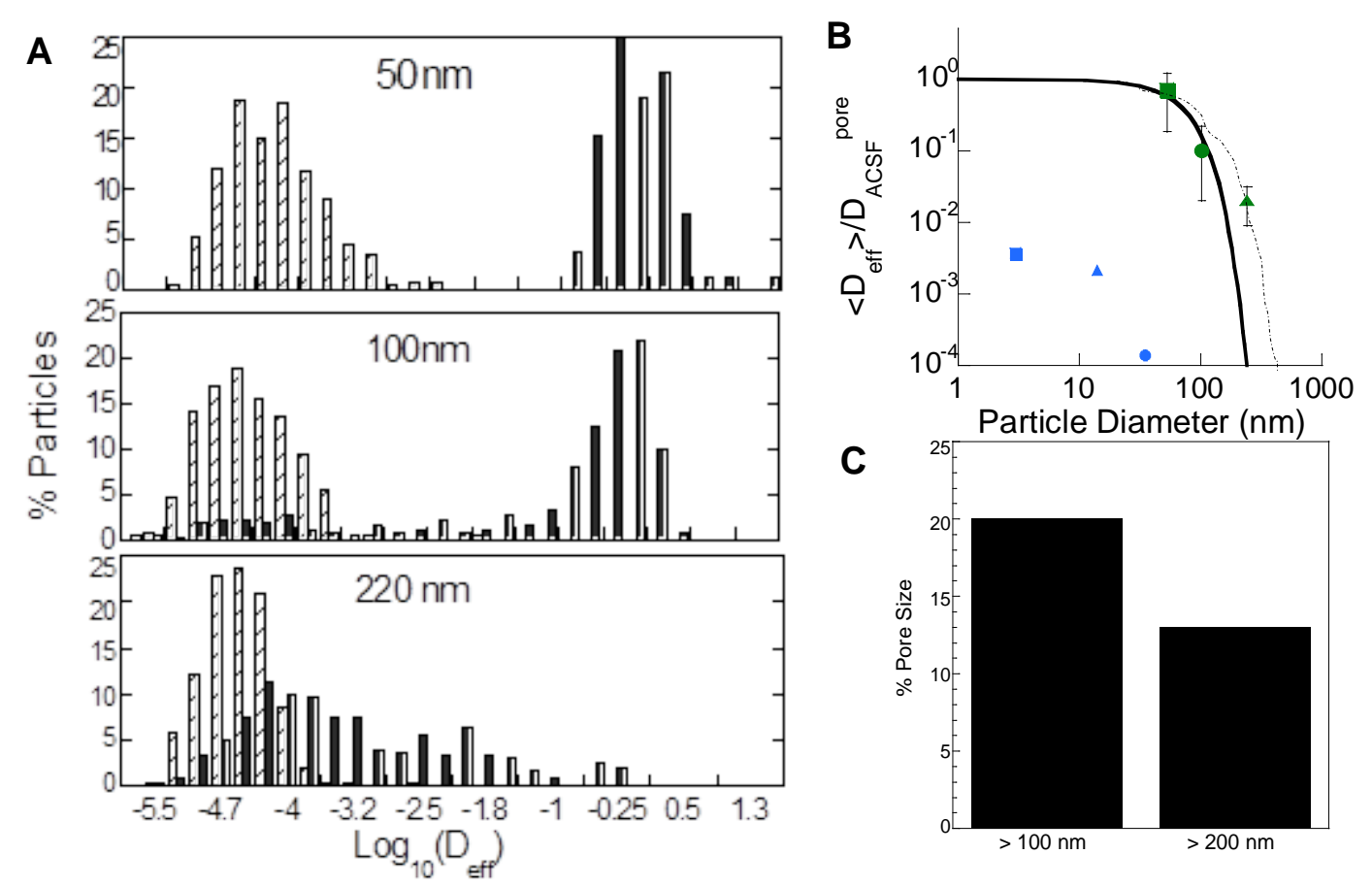

Figure 1.2: Prediction of normal brain extracellular spacings based on nanoparticle diffusion. (A) Distribution of the logarithmic Deff of individual nanoparticles ( $\quad$ PS PEG5K and $\square$ PS COOH) at $\mathrm{T}=1 \mathrm{~s}$. Data represents 4 independent experiments, with an average of $n>100$ nanoparticles per experiment. (B) Normalized ensemble-averaged diffusivities (<Deff $>/ D W$ ) for different sized PEG-coated PS particles $(60 \mathrm{~nm}(\boldsymbol{\bullet}), 110 \mathrm{~nm}(\bullet)$, and $240 \mathrm{~nm}(\boldsymbol{\Delta}))$ at $\mathrm{T}=1 \mathrm{~s}$, where DW is the theoretical diffusivities of neutral particles in PBS. The solid and dashed lines represent the range of theoretical <Deff $>$ /DW ratios for various sized 60-, 110-, and 240-nm particles predicted by the obstruction scaling model. This leads to an estimated mesh spacing range in normal rat brain tissue of $20-230 \mathrm{~nm}$ obtained by maximum likelihood estimation fitting to experimental diffusivity ratios. <Deff>/DW from Thorne et al [28] are provided (3 nm $(\boldsymbol{\bullet}), 14 \mathrm{~nm}(\mathbf{\Delta})$, and $35 \mathrm{~nm}(\bullet))$ for reference to previously estimated values. (C) Percent pores larger than 100- and 200-nm. Data represents the ensemble average of four independent experiments with $n>100$ particles tracked for each experiment. 


\section{VERIFYING BBB DISRUPTION FOLLOWING FUS TREATMENT}

To achieve quasi-real time verification of BBB disruption following FUS treatment, gadolinium (Magnevist $\odot, 0.1 \mathrm{~mL} / 200 \mathrm{~g}$ ) was delivered intravenously immediately following sonication. T1-weighted images (Fig1.3A) were captured to visualize BBB disruption. Images taken from the coronal (Fig1.3A, middle column) and sagittal slices (Fig1.3A, rightmost column) indicate approximately $2.35 \mathrm{~mm}^{3}$ of BBB disruption $\left(\pi \times 0.5 \mathrm{~mm}^{2} \times 3 \mathrm{~mm}\right)$, which agrees with the beam plots of the transducer provided by the manufacturer. Quantitative analysis of the coronal images demonstrates significant differences between the two FUS pressures used. Higher FUS pressure (0.6 $\mathrm{MPa}$ ) produced a significant 2.3-fold increase in the total area of the enhanced region (Fig1.3B) as well as a more moderate significant increase in grayscale intensity within the contrast enhanced focal regions (Fig1.3C) when compared to 0.4 MPa. While variations in both the size and intensity of focal spot enhancement were detected at the same pressure, these variations were not significant and care was taken to minimize spatial differences by selecting focal regions in relatively homogeneous areas of the brain, avoiding ventricles and the cerebellum.

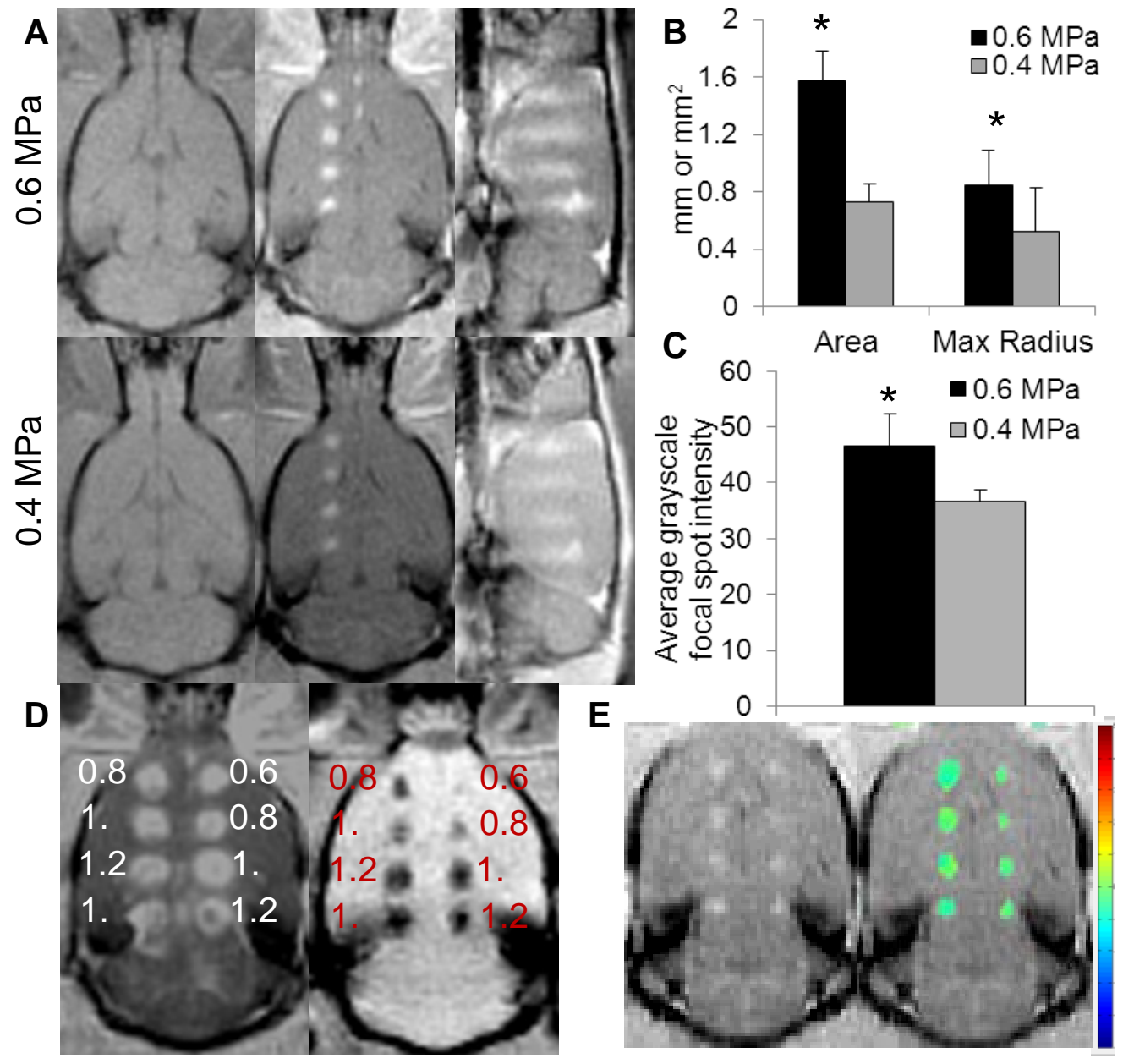

Figure 1.3: FUS-mediated gadolinium delivery and hemorrhage formation in the rat brain are dependent on ultrasound pressure. (A) MRgFUS-mediated BBB disruption, measured by gadolinium delivery, is enhanced at $0.6 \mathrm{MPa}$ compared to $0.4 \mathrm{MPa}$, shown by high resolution T1-weighted MR 
images. Left column, pre-sonication coronal images. Middle column, post-sonication contrast enhanced coronal images. Right column, post-sonication contrast enhanced sagittal images. (B,C) $0.6 \mathrm{MPa}$ produced significantly larger contrast enhanced focal regions and also increased the average grayscale intensity within the focal regions (C), indicating that both area and degree of BBB disruption was increased at $0.6 \mathrm{MPa}(\mathrm{n}=8$, measurements taken from coronal images). (D) Erythrocyte leakage across the BBB , as detected by T2*-weighted MR images, was also pressure dependent. Hypointense regions, indicating the presence of blood in the tissue, appeared at $92 \%$ of locations $(n=39)$ sonicated at or above $0.8 \mathrm{MPa}$ while only $16 \%$ of locations $(n=43)$ sonicated at $0.6 \mathrm{MPa}$ showed a loss in signal. Hypointense regions produced at $0.6 \mathrm{MPa}$ were both smaller and fainter than those produced at higher pressures. Contrast-enhanced T1-weighted image (left) is shown alongside T2*-weighted image (right). Overlaid numbers denote the pressure at which each location was sonicated, in MPa. (E) MR thermometry, performed at both 0.4 MPa (right hemisphere) and 0.6 MPa (left hemisphere) in 2 animals, indicated that neither pressure produced a focal temperature rise. Contrast enhanced T1-weighted image (left) shown alongside thermometry results. Temperature scale bar $\pm 5^{\circ} \mathrm{C}$. * indicates $p<0.05 . n \geq 4$ or as noted. ${ }^{177}$

\section{DETERMINING SAFETY THRESHOLDS FOR FUS TREATMENT}

We performed two MR-based safety studies on additional animals. T2* weighted MR images, which detect red blood cell leakage across the BBB, were taken 2-5 minutes post-sonication at pressures ranging from 0.4 to $2.0 \mathrm{MPa}$. Hypointense regions indicating the presence of blood in the tissue appeared at $92 \%$ of locations $(n=39)$ sonicated with pressures greater than or equal to $0.8 \mathrm{MPa}$, while only $16 \%$ of locations $(n=43)$ sonicated with 0.6 MPa developed hypointensity (Fig1.3D). No erythrocyte leakage was detected at $0.4 \mathrm{MPa}(\mathrm{n}=3)$. Of note, hypointense regions produced at 0.6 $\mathrm{MPa}$ were much smaller and fainter than those produced at higher pressures, and only occurred when sonications were performed close to the olfactory bulb.

\section{BPN DISTRIBUTION IN THE BRAIN FOLLOWING MRGFUS}

Analysis of brain sections following FUS treatment indicated that $60 \mathrm{~nm}$ PS-PEG demonstrated a FUS pressure dependent delivery trend (Figs1.4,1.5). 0.6 MPa significantly increased BPN delivery to the interstitium at 1 hour, compared to $0.4 \mathrm{MPa}$ (Fig1.4, 1.5A). At 24 hours, over $60 \%$ of BPNs in the sonicated region had been delivered to the interstitium (Fig1.5A). Control regions showed no BPN delivery (Fig1.4). At 1 hour post-sonication, higher US pressure of $0.6 \mathrm{MPa}$ doubled the area of brain parenchyma showing fluorescent enhancement due to NP delivery surrounding a single vessel, defined as a NP "cloud", compared to NP coverage at 0.4MPa (Fig1.5B). A comparison of the percentage of blood vessels in the focal region showing either endothelial cell NP delivery or the production of a NP cloud (collectively termed "NP" Vessels") showed no significant difference, while the percentage of these $\mathrm{NP}^{+}$Vessels that had produced NP clouds was increased $(p<0.05)$ to $50 \%$ at the higher US pressure (Fig1.5C). The NP clouds were further separated into small and large clouds using a 200 square micron size cutoff based on the average vessel spacing in the brain sections. The higher US pressure produced a significant $(p<0.01) 4.6$-fold increase in large NP clouds and tripling NP coverage within the sonicated region. 


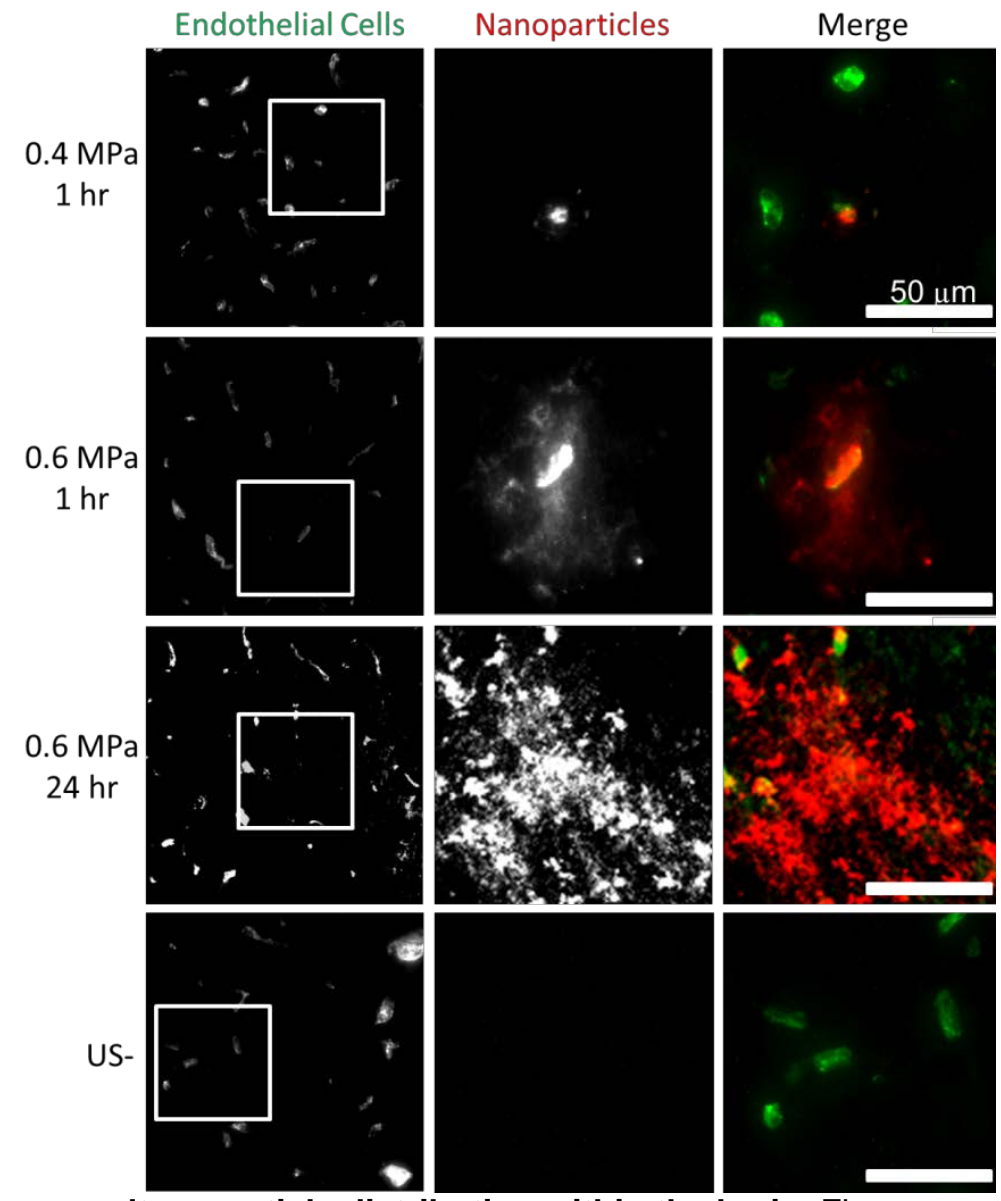

Figure 1.4: FUS pressure alters particle distribution within the brain. Fluorescent images demonstrate the delivery of fluorescently labeled BPNs to the brain parenchyma at both 0.4 and $0.6 \mathrm{MPa}$ 1 hour and 24 hours post-sonication. BPNs were delivered to both the endothelial cells lining the vasculature and the interstitial space surrounding the vessels in sonicated regions. Control regions showed no NP delivery. Scale bars, $50 \mu \mathrm{m}$. $\mathrm{n} \geq 5$. Adapted from ${ }^{177}$

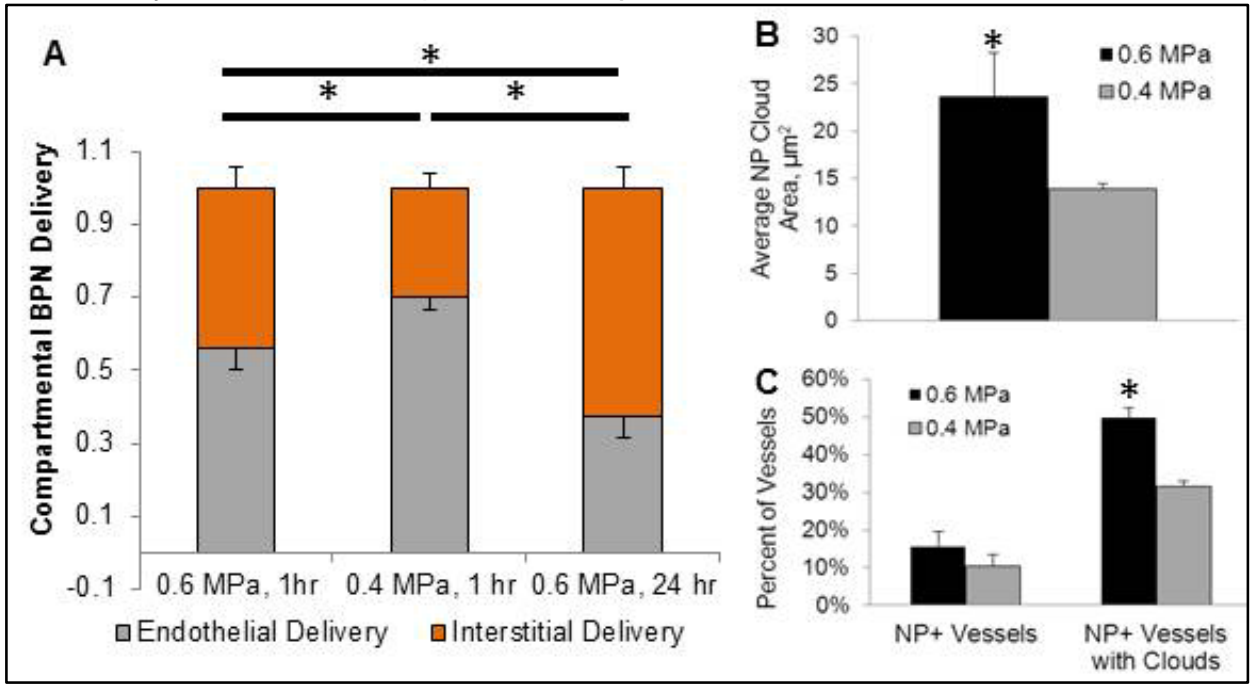

Figure 1.5: MRgFUS-mediated delivery of $60 \mathrm{~nm}$ long circulating brain-penetrating nanoparticles is pressure and time dependent. (A) BPN delivery is shifted towards the interstitial space with higher FUS pressure, and interstitial delivery increases between 1 and 24 hours post sonication. (B) NP cloud size, defined as the area of thresholded BPN fluorescent intensity outside of the vasculature, is two-fold higher 
at 0.6 MPa. (D) The percentage of NP+ vessels, defined as any vessel showing BPN delivery to endothelial cells or the interstitium, was near $15 \%$ for both pressures, but at $0.6 \mathrm{MPa}, 50 \%$ of the NP ${ }^{+}$ vessels had delivered BPNs to the brain parenchyma ("NP+ Vessel with Clouds"), compared to only $30 \%$ at $0.4 \mathrm{MPa}$. * denotes $\mathrm{p}>0.05$. $\mathrm{n} \geq 5$.Adapted from ${ }^{177}$

\section{BIODEGRADABLE BPN DISTRIBUTION IN THE BRAIN FOLLOWING MRGFUS}

We demonstrate that a clinically relevant, biodegradable polymer nanoparticle can successfully reach the brain with this approach. We have previously shown that poly(lactic-co-glycolic) acid (PLGA)-PEG NPs (PLGA-PEG) were similar in size and surface charge to PS-PEG NPs and were able to diffuse in normal brain tissue ${ }^{169}$. Following the MRgFUS protocol described above for PS-PEG NPs, we demonstrate delivery of 75-nm PLGA-PEG NPs in the brain in regions where MRgFUS was applied following intravenous administration of PLGA-PEG NPS. NP clouds comparable to those produced by 60nm PS-PEG NPs are clearly visible (Fig1.6).
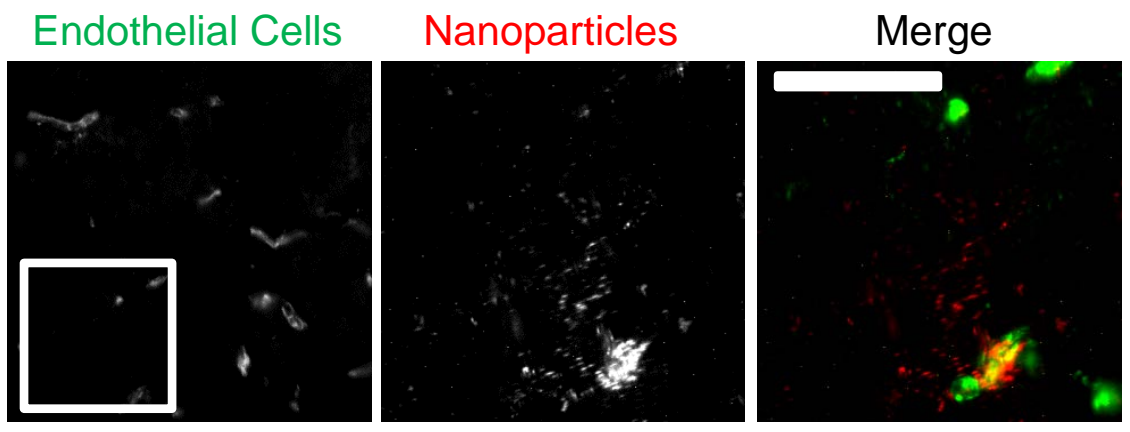

Figure 1.6: Biodegradable PLGA-PEG NPs distribute in the brain following MRgFUS.

(A) Fluorescent images show delivery of fluorescent biodegradable 75-nm PLGA-PEG particles to both the endothelium and the interstitial space in sonicated regions, as with PS-PEG NPs. FUS pressure was $0.6 \mathrm{MPa}$. Control regions without MRgFUS showed no NP delivery (not shown). Scale bar $=50 \mu \mathrm{m} . \mathrm{n}=5$. 270

\section{CORRELATING BPN DELIVERY WITH GADOLINIUM ENHANCEMENT}

We retrospectively sought to determine whether our high-resolution T1-weighted contrast enhanced coronal MRI images correlate with our fluorescent microscopy NP delivery data. The average grayscale focal spot intensity calculated from the MRI images predicted both the NP clouds per field of view $\left(R^{2}=0.90\right.$, Fig1.7A) and the percent of vessels with NP clouds $\left(R^{2}=0.79\right.$, Fig1.7B). Each data point corresponds to a single animal, 4 sonicated at $0.6 \mathrm{MPa}$ and 3 sonicated at $0.4 \mathrm{MPa}$. 

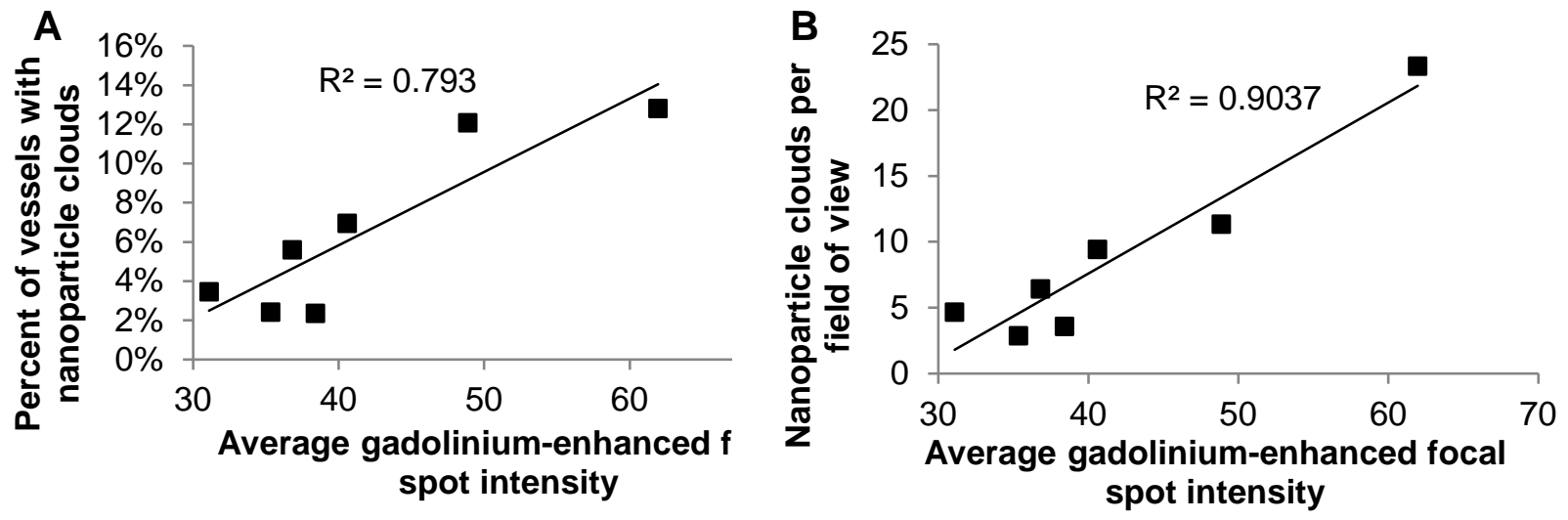

Figure 1.7: Gadolinium delivery predicts BPN delivery in MRgFUS-mediated BBB disruption. (A, B) Gadolinium delivery, measured by the average grayscale focal spot intensity calculated from coronal contrast-enhanced T1-weighted MR scans, was found to correlate with the average number of BPN clouds per field of view (A) and the percent of vessels that produced BPN clouds (B), measured from fluorescent imaging of sectioned brains. Each point on the plots represents an animal, 4 sonicated at 0.6 $\mathrm{MPa}$ and 3 sonicated at $0.4 \mathrm{MPa}$. Each animal was sonicated in four locations and metrics were averaged across sonications. ${ }^{177}$

\section{STATISTICAL ANALYSIS}

Data in Figures 1.3 and 1.5 were analyzed using an independent-samples t-test in Microsoft Excel (2010). Data in figure 1.7 was analyzed using linear regression in Microsoft Excel (2010). For all experiments, significance was defined as $\mathrm{p}<0.05$.

\section{DISCUSSION AND CONCLUSIONS}

We show here that MRgFUS and MBs can be used to safely deliver $60 \mathrm{~nm}$ PSPEG NPs and 75-nm PLGA-PEG across the BBB in vivo. Feasibility studies that analyzed the delivery of gadolinium, an MR contrast agent, across the BBB after FUS demonstrated that higher ultrasound pressures deliver more gadolinium across a greater area. This trend translated to densely PEG-coated PS-NPS (BPN), which we show can circulate and accumulate in the brain in regions where the BBB is disrupted by FUS and MBs. MRgFUS-mediated delivery of the 60-nm PS-PEG showed that higher FUS pressure shifts the distribution of the BPNs within the brain tissue, producing greater parenchymal coverage and enhancing the penetration of the PS-PEG NPs. Furthermore, these particles continue to diffuse after delivery, as demonstrated by the increase in parenchymal delivery at 24 hours compared to 1 hour. T2* weighted MRI imaging indicated little to no blood pooling at $0.6 \mathrm{MPa}$, demonstrating the safety of using FUS for BPN delivery in the normal brain. Gadolinium enhancement post-sonication correlated with NP delivery, providing a non-invasive metric for predicting NP delivery. Finally, we also show that MRgFUS is able to deliver 75-nm biodegradable PLGA-PEG NPs across the BBB, bringing this technology one step closer to the clinic. Ultimately, we conclude that MRI guided opening of the BBB using FUS and MBs is a promising strategy for enhancing the delivery of polymeric nanoparticles into the normal brain. This approach has the potential to become a platform technology for treating a broad 
spectrum of CNS disorders, offering the targeted delivery of a host of therapeutics, including drugs, genes, siRNAs, and miRNAs.

Strategies to promote nanoparticle transit across the BBB can be divided into two broad areas: surface modification to promote nanoparticle uptake by an intact BBB 271,272 and techniques to disrupt or circumvent the BBB ${ }^{136}$, including FUS. Recently, several groups have explored the use of surfactants such as polysorbate 80 (P80), poloxamer 407 (F127), and poloxamer 188 (F68) to coat nanoparticle surfaces and promote crossings of the BBB ${ }^{273,274}$. Kulkarni and Feng compared the effect of several surfactants on delivery of systemically administered poly(lactic-co-glycolic acid) (PLGA) nanoparticles across the BBB. They reported that, while F68, F127, and P80 coatings enhanced crossing of the BBB, F68-coated particles accumulated in the highest concentrations in the brain compared to particles with F127, P80 and other surfactant coatings ${ }^{274}$. Kreuter et al have shown that P80 and F68 coated nanoparticles undergo endocytosis followed by transcytosis, leading to uptake in the BBB endothelial cells. P80-coated nanoparticles were shown to inhibit growth of gliomas in rats due to increased brain uptake compared to uncoated particles ${ }^{275}$. F68 coated particles have also been shown to improve efficacy against intracranial glioblastomas (GBMs) compared to uncoated particles ${ }^{276,277}$. A recent publication using poly(vinyl alcohol) (PVA) coated paclitaxel loaded PLGA particles administered through CED showed enhanced survival in glioma bearing rats ${ }^{278}$. Although surfactants such as P80, F127, F68, and PVA have been widely used to increase nanoparticle uptake into the brain, these particles are poorly diffusive and prone to cell uptake, limiting their ability to reach distant diseased cells and emphasizing the need for a novel nanoparticle and delivery system design that is tailored to the brain.

MRgFUS has been applied with intravenous delivery of doxorubicin (Dox)-loaded liposomal nanoparticles to improve antitumor efficacy ${ }^{3}$. While significant improvement in tumor growth delay was obtained, the authors state the enhanced therapeutic effect may be due to release of Dox from disrupted liposomes near the focal site, as opposed to intact liposomes penetrating the brain parenchyma following MRgFUS-mediated delivery. Additionally, liposomes have well-documented limitations as controlled sustained delivery vehicles when compared to polymeric nanoparticles ${ }^{179}$. More recently, intravenously delivered antibody conjugated biocompatible gold nanoparticles have been shown to accumulate in the brain with MRgFUS, but only in the absence of liver macrophages ${ }^{279}$. In our study, we combine surface-modified nanoparticles that can penetrate in brain parenchyma with a FUS technique to disrupt the BBB, in an attempt to improve the delivery of therapeutically relevant nanoparticles to the CNS. We have previously shown PS-PEG NPS up to 114-nm can diffuse within normal human tissue 169. The results obtained with MRgFUS of PS-PEG NPs can be translated to PLGA-PEG NPS, representing a biodegradable, clinically relevant polymer nanoparticle platform.

We showed that MRgFUS can deliver BPNs to both the vessel endothelium and the brain parenchyma without any uptake in non-targeted regions. Higher FUS pressure (0.6 MPa vs 0.4 MPa) increased BPN coverage within the interstitium, as well as the fraction of NPs delivered beyond the vessel endothelium. The percentage of capillaries 
exhibiting BPN delivery in sonicated regions in our study appeared similar to the results from other studies in which fluorescent dextran delivery across the BBB via MB activation with FUS was observed using multiphoton or 2-photon microscopy techniques 63. The increase in NP coverage within the parenchyma observed at 24 hours is likely due to a combination of enhanced delivery (via transcytosis or tight junction disruption) and increased diffusion as the BPN continued to diffuse away from the vessels. There are many variables that can be altered to enhance NP delivery. The characteristics of the MB population may be an important factor influencing heterogeneity in BBB disruption. Many studies, including our own work ${ }^{280}$, have shown that MB size ${ }^{281}$, concentration ${ }^{282}$ and behavior ${ }^{283}$ have an impact on vessel permeabilization. The cavitation threshold changes with MB size ${ }^{284}$, so the use of a polydisperse MB population can cause variation in the vessel response to sonication, and therefore, NP delivery. More uniform BBB opening could be achieved through the use of size-sorted MBs ${ }^{66}$. Another potential factor contributing to disparate NP delivery is the inherent variation in vessel density in the brain ${ }^{285}$. Future studies could utilize different MB populations to increase the delivered NP dose to within the therapeutic window.

BPN size is also a possible variable that can be optimized for drug delivery to the brain. Due to the ability of $110 \mathrm{~nm}$ PS-PEG particles to penetrate and circulate (data not shown), larger particles with a higher payload might increase the drug dose delivered to the brain with FUS and MB. Extravasation across an impaired or disrupted BBB is size dependent, as demonstrated using Evans Blue, various molecular weight FITCconjugated dextrans, and other small molecular markers 286,287 . Although particles larger than 100-nm can diffuse in the extracellular matrix in normal brain tissue, the extracellular matrix spacing is significantly decreased in most diseased states ${ }^{167}$. Therefore, we used 60-nm PS-PEG combined with FUS to ensure passage across a disrupted BBB, and to aid in translation to a clinically relevant disease model and biodegradable platform. PLGA-PEG NPs, around 75-nm in size, were also able to accumulate in brain, suggesting that a therapeutically relevant platform can be delivered to the brain with this method. Various size BPNs will need to be tested with multiple FUS pressures and MB diameters to determine the highest percent of BPN uptake and penetration within the brain parenchyma, while minimizing tissue damage and additional side effects.

Depending on frequency, pressure, and MB diameter, endothelial barriers can be opened with or without microvessel damage (i.e. irreversible vs. reversible BBB opening), indicating that different modes of BBB opening are possible $60,62,288,289$. Other investigators have presented compelling evidence that reversible BBB opening is associated with a stable MB cavitation mode, while irreversible BBB opening appears to occur above the threshold for inertial cavitation ${ }^{48,71,281}$. Cavitation detection was not used here; however, we did use MR-based methods to assess protocol safety. Using T2* weighted MRI, blood pooling was detected in only $16 \%$ of the sonications performed at 0.6 MPa. Meanwhile, raising peak-negative pressure to $0.8 \mathrm{MPa}$ resulted in a $92 \%$ probability of blood pooling. Together, these results indicate that the transition from stable to inertial cavitation in our system likely occurred somewhere between 0.6 and $0.8 \mathrm{MPa}$. Interestingly, 0.6 MPa sonications that produced blood pooling were always 
located at the most anterior location of the brain we could access with our system. This observation points to variations in skull thickness and geometry, as well as potential differences in vascular density, as significant determinants of the mode of BBB opening. Cavitation detection devices may be incorporated into FUS systems to account for inhomogeneity of the skull and brain tissue. Ultimately, for some CNS applications, low levels of tissue damage may be acceptable if a therapeutic dose of particles can be delivered without an impact on neurological function. Finally, it is also important to note that, in our studies, MR thermometry showed no detectable change in temperature with this FUS pulsing protocol, so there is little to no concern that this protocol will induce thermal damage.

The FDA has approved FUS for thermal tissue ablation in the brain for the treatment of essential tremor, and clinical trials for Parkinson's and glioblastoma multiforme are underway and may be used as a precedent for translating this work to the clinic. Broadly speaking, this study opens the door for delivery of drug-loaded biodegradable nanoparticles across the BBB for many CNS applications, including Parkinson's, depression, brain tumors, epilepsy, and compulsive disorders. While some CNS disorders exhibit a dysfunctional BBB, it is often necessary to deliver therapeutics to areas of the brain with normal BBB function, as is the case in GBM when invasive cells lie beyond the tumor boundary. BPN delivery with FUS and MBs can further improve antitumor efficacy by: (i) providing deeper brain tissue penetration of drug than is achieved with polymer implants (located only at the periphery in the surgical cavity), (ii) enabling multiple dosing regimens without the need for repeat surgery, (iii) facilitating local delivery for tumors with high surgical risk, and (iv) permitting patient-specific BPN design based on the unique tumor subtype and mutations. MR guidance adds the ability to identify and target specific regions [45], reducing off-target toxicity within the brain.

\section{FUTURE DIRECTIONS}

This work demonstrates the feasibility of combining MRgFUS with large polymeric nanoparticles for therapeutic delivery across the BBB. Here, we show that MRgFUS pressure dictates the distribution of large particles after treatment. While three different mechanisms (TJ disruption, transcytosis and sonoporation) have been proposed to explain MRgFUS-mediated BBBD ${ }^{61,126,290}$ and it is generally accepted that all three mechanisms occur simultaneously, our work ${ }^{177}$ and that of others ${ }^{63,291}$ has demonstrated a pressure and vessel diameter-dependent variance in particle delivery. We have shown ${ }^{177}$ that lower MRgFUS pressure $(0.4 \mathrm{MPa})$ preferentially delivers particles to the endothelium, and two-photon microscopy during FUS-mediated BBBD revealed that smaller vessels exhibit rapid particle leakage following BBBD, while larger vessels transport particles to the parenchyma over a longer period of time $[63,207]$. Moreover, larger dextrans were more likely to be transported via the slow mechanism 63,291 . This data, taken together, implies that different BBBD mechanisms may dominate for different particle sizes and applied FUS pressures. Further experimentation utilizing a wider range of FUS pressures and particle sizes could be used to explore this 
phenomenon, and additional measures (genetic knockouts, pharmaceutical manipulation, etc) may also be helpful in isolating delivery mechanisms.

Safe, localized and reversible BBBD with MRgFUS offers a significant advantage over current methods to bypass the BBB and extends the use of many drugs that do not cross the BBB ${ }^{292-294}$. Large, biodegradable polymeric nanoparticle delivery systems can provide sustained drug release while limiting both systemic and local toxicities ${ }^{13}$. Combined, they present a safe, repeatable, non-invasive method to deliver drugs to the brain. This delivery strategy could be used in a wide range of CNS disorders, including primary and metastatic cancers, Alzheimer's and Parkinson's, and depression and OCD 270. Perhaps one of the most immediate applications for this technology is the treatment of GBM following surgical resection of the tumor bulk. GBM is a notoriously aggressive and invasive cancer, with $90 \%$ of patients experiencing tumor recurrence and five-year survival rates hovering below $15 \%{ }^{295}$. Invasive tumor cells often lie within normal tissue protected by the BBB and beyond the reach of traditional therapies. MRgFUS can be combined with drug-loaded biodegradable polymeric nanoparticles to provide localized drug delivery to the infiltrated tissue surrounding the tumor bulk to prevent recurrence and improve patient outcomes.

Safe, non-invasive, repeatable delivery methods to the brain are of even greater import in patients with chronic disease ${ }^{296}$. To reduce the total number of treatment, loading polymeric nanoparticle systems with gene vectors may provide sustained benefits. MRgFUS in combination with gene therapy has been effective in animal studies ${ }^{270}$ and may be the most logical approach for chronic disease in the future. 
Developing an MR-guided focused ultrasound protocol that increases the delivery and efficacy of biodegradable, drug-loaded brain penetrating nanoparticles in a rat model of glioblastoma.

While FUS is capable of improving drug delivery to tumors, it is unclear whether the delivery of $60 \mathrm{~nm}$, systemically administered drug loaded polymeric nanoparticles will provide effective tumor growth control. MR-guided FUS and MBs will be used to disrupt the blood-tumor barrier following intravenous injection of drug-loaded BPNs. Tumor growth will be monitored using contrast-enhanced MRI.

Parts of this chapter are taken from the unpublished manuscript Zhang C, Nance E, P Mastorakos Local Administration of Cisplatin Nanoparticles for Treatment of Malignant Glioma.

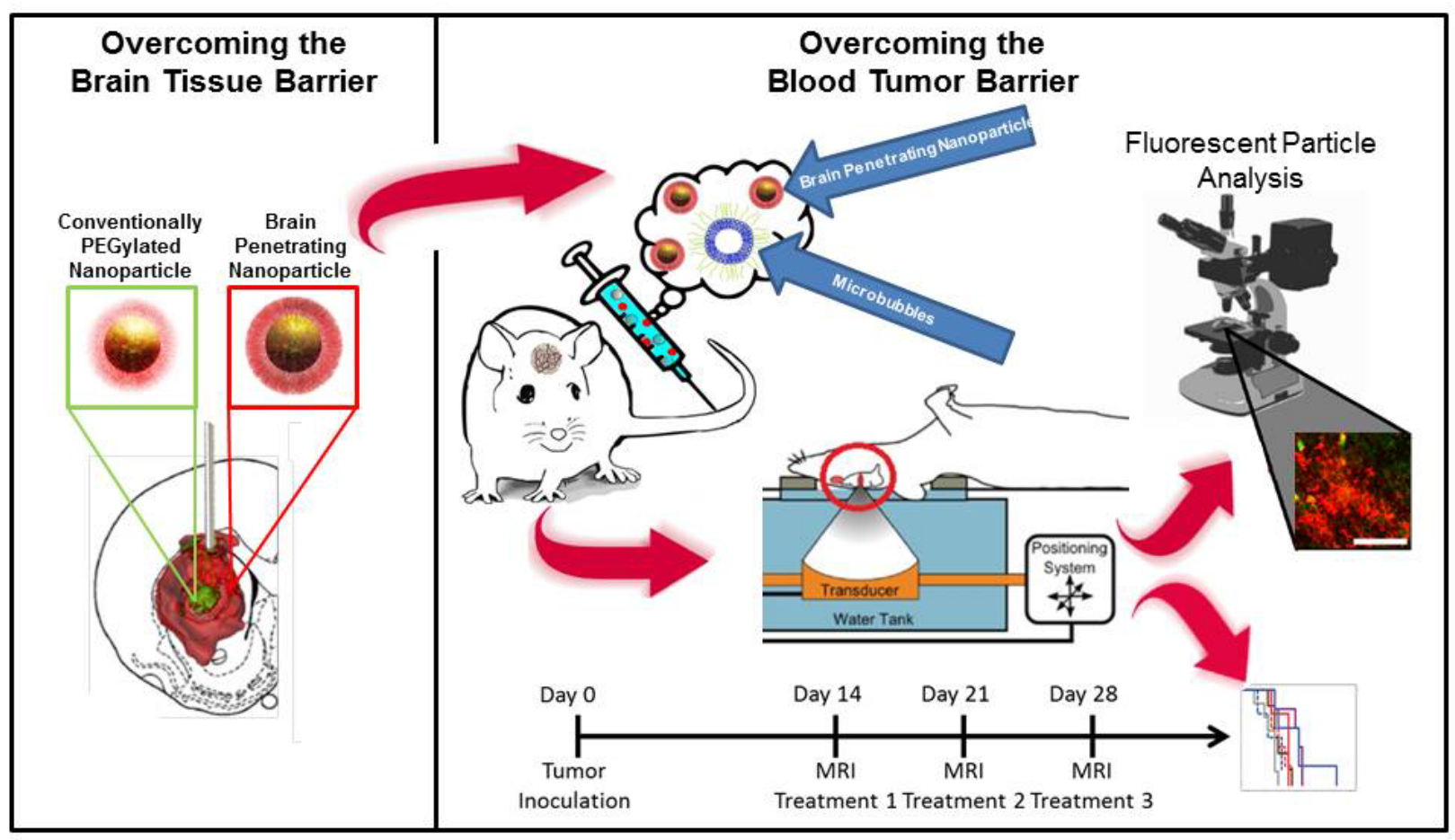


Glioblastoma multiforme (GBM) is an aggressive brain tumor that accounts for $67 \%$ of all primary brain tumors ${ }^{220}$. Due to the highly invasive nature of the disease, cancer cells often lie beyond the visible tumor boundary, which makes full surgical resection difficult ${ }^{297} .90 \%$ of patients develop tumor recurrence at or near the surgical site and many tumors develop drug resistance ${ }^{136}$. Despite advances in drug development, the five year survival rate for GBM is $12 \%$ and has remained virtually unchanged over the past decade ${ }^{220}$.

Cis-diamminedichloroplatinum (Cisplatin, CDDP), a powerful chemotherapeutic, has been effective as a first-line therapy for several types of cancer, including testicular, ovarian, bladder and lung cancers ${ }^{220}$, and has also been used as an adjuvant in the treatment of pediatric brain tumors ${ }^{298}$. However, adult GBM patients treated with CDDP suffered severe nephron- and neurotoxicity even at sub-therapeutic drug concentrations 299-301. Methods that reduce off-target toxicity and/or increase local delivery to permit a decrease in systemic dose would greatly increase CDDP's utility in the treatment of brain tumors ${ }^{299}$.

While direct injection of CDDP can improve therapeutic outcomes, this requires an invasive procedure and still produces significant neurotoxicity ${ }^{302}$. Biodegradable nanoparticle formulations can shield healthy tissues from the toxic effects of CDDP, especially during systemic administration, while enhancing therapeutic efficacy 220,303 . When combined with a strategy to increase the NP concentration at the target site, this drug delivery approach provides the ability to achieve sustained therapeutic CDDP levels in the tumor while minimizing both systemic and local off-target effects.

However, drug delivery to the brain presents unique difficulties ${ }^{304}$. Invasive cancer cells exist within otherwise healthy brain tissue, where they are protected from systemically administered drugs by the blood-brain barrier (BBB). The BBB regulates the transport of most molecules to and from the brain and prevents the vast majority of CNS therapeutics from reaching their target ${ }^{305,306}$. The BBB within the tumor may be impaired, but this impairment is often heterogeneous ${ }^{297,307}$ and produces an unfavorable pressure gradient for drug penetration ${ }^{171}$. Additionally, brain tumors have a higher cell density and collagen content than normal brain, further limiting drug penetration ${ }^{170,308}$. Together these factors contribute to the blood-tumor barrier (BTB).

Transcranial MR-guided focused ultrasound (MRgFUS) is currently the only treatment modality capable of achieving safe, non-invasive, reversible BBB and BTB disruption in a targeted manner ${ }^{71,309-311}$. Once therapeutics cross the BBB or BTB, they must penetrate the complex brain extracellular space (ECS). (Refer to the background section or Aim 1 for additional details.) To address this challenge, we developed "brain penetrating nanoparticles" (BPNs) by adding dense coats of poly(ethylene glycol) (PEG) which enable particles up to $114 \mathrm{~nm}{ }^{169}$ and $70 \mathrm{~nm}{ }^{178}$ in size to penetrate and diffuse within normal brain parenchyma and brain tumors, respectively. 
The ideal method to deliver drugs to the CNS would include (i) a long circulating nanoparticle that can avoid rapid clearance by the reticuloendothelial system (RES), (ii) a noninvasive approach to bypass the BBB, (iii) the ability of the nanoparticle to penetrate within the brain parenchyma and (iv) provide sustained release of a therapeutic agent at the sites of disease. In this chapter, we test the hypothesis that MRgFUS will increase the delivery of BPNs across the BBB/BTB and improve tumor growth control and animal survival in an intracranial rat model of GBM. We use both fluorescently labelled PEGylated polystyrene (PS-PEG) BPNs and biodegradable polyasparticacid (PAA) cisplatin-loaded BPNs (CDDP-BPN) to evaluate the efficacy of this combination therapy. We show that MRgFUS significantly improves BPN delivery and distribution in the tumor, and MRgFUS in combination with CDDP-BPN improves tumor growth control and animal survival. This is the first MRgFUS study demonstrating efficacy with a systemically administered biodegradable polymeric nanoparticle in the treatment of GBM. With the recent FDA approval for the treatment of essential tremor, MRgFUS applications for CNS disorders offer great promise.

\section{EXPERIMENTAL METHODS}

\section{STUDY DESIGN}

\section{BPN DELIVERY}

To test the hypothesis that focused ultrasound can enhance BPN delivery across the blood-tumor barrier, two different cell lines were used: 9L and F98. 9L is a wellcharacterized glioma model, but is highly immunogenic and poorly infiltrative, and thus does not readily recapitulate the characteristics of human gliomas ${ }^{312-314}$. In contrast F98 gliomas are poorly immunogenic and highly infiltrative, more closely replicating the behavior of gliomas in patients ${ }^{312-314}$. It is also more sensitive to CDDP ${ }^{315}$. $9 \mathrm{~L}$ cells were implanted in Sprague-Dawley rats and F98 cells were implanted in Fischer 344 rats as described below. All animals were $160 \mathrm{~g}-180 \mathrm{~g}$ females from the same vendor (Envigo). Fluorescently labelled PS-PEG BPNs developed by the Hanes lab at Johns Hopkins University were delivered intravenously at a dose of $15 \mu \mathrm{g} / \mathrm{g}$ body weight immediately prior to sonication. Tumors were sonicated with a 9 spot grid as described in the following sections. Animals were euthanized 24 hours after FUS treatment with pentobarbital ( $0.5 \mu \mathrm{l} / \mathrm{g}$ body weight). All animal experiments were carried out at the University of Virginia following National Institutes of Health guidelines and local Institutional Animal Care and Use Committee regulations.

\section{DRUG DELIVERY}

To test the hypothesis that ultrasound can enhance the efficacy of drug-loaded BPN in the treatment of glioblastoma, female 160-180g Fischer 344 rats (Envigo) were inoculated with F98 glioma cells and randomly assigned to one of seven groups: 0.8 MPa FUS (FUShi) + CDDP-BPN, FUShi + CDDP, 0.6 MPa FUS (FUSlo) + CDDP-BPN, FUSlo + CDDP, CDDP-BPN, CDDP, and control. Animals were kept on a 12/12 hr 
light/dark light cycle in a temperature and humidity controlled environment and were provided with food and water ad libitum. Animals were imaged using contrast (Gd)enhanced T1 weighted MRI weekly beginning 1 week after inoculation, and 3 weekly treatments began as soon as the tumors became visible (typically week 1-2). Surviving animals were imaged weekly following the final treatment. Therapeutics (CDDP-BPN or CDDP) were delivered intravenously at a dose of $2.5 \mathrm{mg} / \mathrm{kg}$ CDDP (determination of maximum tolerated dose is provided later in this section). An additional set of animals was used to evaluate the delivery of fluorescently labelled CDDP-BPN. These animals were treated once and euthanized 24 hours after treatment. All animals were weighed and evaluated based on appearance, behavior and locomotion weekly to monitor tumor progression. When animals reached approved humane endpoint criteria, they were euthanized with pentobarbital $(0.5 \mu \mathrm{l} / \mathrm{g}$ body weight). All animal experiments were carried out at the University of Virginia following National Institutes of Health guidelines and local Institutional Animal Care and Use Committee regulations.

\section{MICROBUBBLE FABRICATION AND CHARACTERIZATION}

As described in Aim 1.

PS-PEG NANOPARTICLE PREPARATION AND CHARACTERIZATION

As described in Aim 1.

POLYMER LABELING WITH A FLUORESCENT DYE

The following work was performed at Johns Hopkins University.

For fluorescent labeling of polymer, AlexaFluor 647 Cadaverine (AF647; ThermoFisher Scientific) was conjugated to PAA in $200 \mathrm{mM}$ borate buffer (pH 8.5) for 72 hours at room temperature. The solution was dialyzed against deionized water using a 20kDa MWCO G2 Dialysis device (Spectrum Lab) for 120 hours, followed by lyophilization. The labeled PAA was stored at $-20^{\circ} \mathrm{C}$ until use.

\section{CDDP-BPN NANOPARTICLE FABRICATION AND CHARACTERIZATION}

The following work was performed at Johns Hopkins University.

Cisplatin-loaded nanoparticles were formulated using a co-polymer of polyaspartic acid (PAA) and polyethyleneglycol (PEG). PAA (MW: $27 \mathrm{kDa}$, Alamanda Polymers, Huntsville, AL) was reacted with PEG (MW: 5 kDa, Creative PEGworks, Winston Salem, NC) at a 1:10 molar ratio with an addition of 1-ethyl-3-(3dimethylaminopropyl) carbodiimide (EDC, Invitrogen, Carlsbad, CA; equimolar with PEG). The reaction was carried out in $200 \mathrm{mM}$ borate buffer (pH 8.5) for 24 hours at room temperature followed by dialysis against deionized water using a $50 \mathrm{kDa}$ MWCO dialysis device (Spectrum Lab, Rancho Dominguez, CA) for 120 hours. The solution 
was lyophilized to obtain a powder of purified PEG-conjugated PAA (PAA-PEG) which was then stored at $-20^{\circ} \mathrm{C}$ until use. The PAA:PEG ratio was confirmed using nuclear magnetic resonance (NMR) to be $\sim 1: 10: 1 \mathrm{H} \mathrm{NMR} \mathrm{(500} \mathrm{MHz,} \mathrm{D2O):} \delta$ 2.70-2.80 (br, $\mathrm{CHCH}_{2} \mathrm{COOH}$ ) 3.55-3.75 (br, $\left.-\mathrm{CH}_{2} \mathrm{CH}_{2} \mathrm{O}-\right)$, 4.40-4.55 (br, $\mathrm{NHCHCH}_{2}-$ ) and 3.3-3.4 (s, $\mathrm{OCH}_{2} \mathrm{CH}_{2} \mathrm{CH}_{3}$ ). A representative NMR spectrum confirming PEGylation of PAA is provided in Fig S1. Immediately prior to nanoparticle (NP) formulation, the lyophilized polymers were dissolved in ultrapure distilled water.

CDDP-loaded NP were formulated using a previously reported method ${ }^{316}$ with a slight modification in order to improve drug loading density. To substitute chloride to hydroxyl, $16.7 \mathrm{mmol}$ of cisplatin (CDDP) was mixed with 2 molar equivalents of silver nitrate $\left(\mathrm{AgNO}_{3}\right)$ in $5 \mathrm{~mL}$ water overnight in the dark. The resulting white turbid solution was centrifuged at $15,000 \mathrm{rpm}$ for 10 minutes and filtered through $0.45 \mathrm{~nm}$ filters to remove $\mathrm{AgCl}$ precipitates. The freshly prepared aquated platinum solution was analyzed to determine the platinum concentration using flameless atomic absorption spectroscopy (AAS) (Perkin Elmer, Waltham, MA). CDDP-BPN were formulated by reacting $5 \mathrm{mM}$ aquated cisplatin with $5 \mathrm{mM}$ aspartic acid at an optimized aspartic acid ratio of PEG-PAA: PAA in RNase-free water. The $\mathrm{pH}$ was adjusted to 6.5-6.8 with $0.1 \mathrm{M}$ $\mathrm{NaOH}$ and shake at $150 \mathrm{rpm}$ for 72 hours at room temperature. Un-PEGylated CDDPloaded NP (CDDP-UPN) were formulated by reacting $5 \mathrm{mM}$ PAA with $2.5 \mathrm{mM}$ cisplatin in RNase-free water for 72 hours. After 72 hours centrifuge particles using 100 microns ultrafilters at $1000 \mathrm{~g}$ for 15 minutes with repeated wash to get rid of free drug. For the formulation of fluorescently labelled particles, AF647-labeled PAA was used instead of unlabeled PAA.

Physicochemical characteristics of NP were determined using a Zetasizer NanoZS (Malvern Instruments, Southborough, MA). All particles were diluted in $10 \mathrm{mM}$ $\mathrm{NaCl}$ (diluted from phosphate buffered saline) and dynamic light scattering (DLS) was employed to determine the hydrodynamic diameter and polydispersity index (PDI) at a backscattering angle of $173^{\circ}$. The $\zeta$-potential, a measure of particle surface charge, was determined using laser Doppler anemometry. Quantification of drug content within the NP was conducted using AAS and the loading density was calculated as the $\%$ mass of drug in the total particle mass. The size and morphology of NP was determined using a Hitachi $\mathrm{H} 7600$ transmission electron microscope (TEM, Hitachi, Japan). NP stability was measured in artificial cerebrospinal fluid (ACSF) and 10\% fetal bovine serum (FBS). Either CDDP-UPN or CDDP-BPN were dissolved in ACSF and/or $10 \% \mathrm{FBS}$ at $37^{\circ} \mathrm{C}$ and hydrodynamic diameters were determined by DLS at different time points after the initiation of incubation.

\section{CDDP RELEASE FROM NANOPARTICLES}

The following work was performed at Johns Hopkins University.

To determine the CDDP release rate over time, CDDP-BPN were dispersed in 2 $\mathrm{mL}$ PBS ( $\mathrm{pH}:$ 7.4) (Harvard Apparatus, Holliston, MA) within a $100 \mathrm{kDa}$ MWCO dialysis tube (Spectrum Lab). The tube was then placed in a $20 \mathrm{~mL}$ PBS sink (simulated infinite sink) and shaken at $37^{\circ} \mathrm{C}$. At specific time points, total sink volume of PBS was replaced 
with fresh PBS and the CDDP content in the sink was determined by AAS to generate a release kinetics curve.

\section{CELL CULTURE}

F98 and 9L rat glioma cells were obtained from the American Type Culture Collection (ATCC). Cells were cultured in high glucose Dulbecco modified Eagle medium (DMEM, Gibco) with $1 \mathrm{mM}$ sodium pyruvate (Gibco) and 10\% fetal bovine serum (FBS, Gibco) added. For each experiment, cells were brought back from cryopreservation to eliminate variations in in vitro passage number. Cells were maintained at $37^{\circ} \mathrm{C}$ and $5 \% \mathrm{CO} 2$.

\section{IN VITRO CELL VIABILITY}

The following work was performed at Johns Hopkins University.

F98 glioma cells were seeded at a concentration of 5,000 cells per well in $100 \mu \mathrm{L}$ of media and allowed to attach overnight in a 96-well plate. The following day, the media was replaced with $100 \mu \mathrm{L}$ of fresh media with $10 \mu \mathrm{L}$ of either carrier-free CDDP or CDDP-BPN ( $25 \mu \mathrm{M}$ to $0.0625 \mu \mathrm{M}$ in 10-fold dilutions). Cells were incubated for 3 days at $37^{\circ} \mathrm{C}$ and $5 \% \mathrm{CO}_{2}$. To quantify the number of live cells, media was replaced with $100 \mu \mathrm{L}$ of fresh medium and $10 \mu \mathrm{L}$ of Dojindo Cell Counting Kit-8 (Dojindo Molecular Technologies, Inc., Rockville, MD) solution was subsequently added. Cells were incubated for 2 hours at $37^{\circ} \mathrm{C}$, followed by the measurement of absorbance at 450 nm using a Synergy Mx Multi-Mode Microplate Reader (Biotek, Instruments Inc. Winooski, VT). The $\%$ cell viability was normalized to the untreated cell control.

\section{NEOCORTICAL SLICE PREPARATION AND MULTIPLE PARTICLE} TRACKING

The following work was performed at Johns Hopkins University.

All animal experiments were carried in accord with local Institutional Animal Care and Use Committee regulations. Healthy rat brain tissue slices were prepared according to a slightly modified protocol of a previous publication ${ }^{316} .1 .5 \mathrm{~mm}$ thick brain tissue slices were prepared using a Zivic Mouse Brain slicer (Zivic instruments, Pittsburgh, PA) and placed in custom-made microscopy chambers. $0.5 \mu \mathrm{L}$ of fluorescently labeled CDDP-UPN or CDDP-BPN was injected at a depth of $1 \mathrm{~mm}$ into the cerebral cortex using a $10 \mu \mathrm{L}$ Hamilton Neuros Syringe (Hamilton, Reno, NV). The chambers were sealed using a coverslip to minimize convective bulk flow so that particle movement could be fully attributed to Brownian diffusion. The transport rates of particles were calculated by analyzing the particle trajectories in brain tissue slices $(\mathrm{N}=3$ for each particle type). The particle trajectories were recorded as 20 second movies at an exposure of $66 \mathrm{~ms}$, using an EMCCD camera (Evolve 512; Photometrics, Tuscon, AZ) mounted on an inverted epifluorescence microscope (Axio Observer D1, Carl Zeiss, 
Hertfordshire, UK) equipped with a 100x oil-immersion objective (NA 1.3). MSD of particles were calculated and compared at a timescale of $\tau=1 \mathrm{~s}$.

\section{FLUORESCENCE-BASED IMAGING OF IN VIVO NP SPREAD}

The following work was performed at Johns Hopkins University.

Fluorescently labeled CDDP-UPN and CDDP-BPN (12 $\mu \mathrm{g}$ of CDDP) at a ratio of 1:1 were loaded in a $50 \mu \mathrm{L}$ Hamilton Neuros Syringe (Hamilton) and co-administered into the striatum of female Fisher 344 rats $(200-220 \mathrm{~g})$ via convection enhanced delivery (CED). A burr hole was drilled $3 \mathrm{~mm}$ lateral and $1 \mathrm{~mm}$ posterior to the bregma. The catheter was vertically mounted on a Chemyx Nanojet Injector Module (Chemyx, Stafford, TX), which was held on a small animal stereotactic frame (Stoelting, Wood Dale, IL). The catheter tip was lowered to a depth of $3.5 \mathrm{~mm}$ and NP were infused at a rate of $0.33 \mu \mathrm{L} / \mathrm{min}$, followed by catheter withdrawal at a rate of $1 \mathrm{~mm} / \mathrm{min}$. Animals were sacrificed 1 hour post-administration and the brains were removed and immediately frozen on dry ice. Tissues were cryosectioned (Leica CM 3050S, Leica Biosystems, Buffalo Grove, II) into successive $100 \mu \mathrm{m}$ coronal slices and imaged using a Zeiss confocal 710 laser scanning microscope through Cy5 (AF647; CDDP-UPN) and Cy3 (AF555; CDDP-BPN) channels. Confocal images of brain slices were quantified for fluorescent distribution of NP within the striatum using a MATLAB script developed in our lab. Fluorescent distribution of NP in the ventricles or white matter tracts (WMT) was not included in the quantification. The area of distribution calculated from each slice was multiplied by the slice thickness of $100 \mu \mathrm{m}$ and summated across all images to obtain a total volume of distribution.

\section{TUMOR INOCULATION}

F98 or $9 \mathrm{~L}$ cells were removed from the culture flask with $0.25 \%$ Trypsin-EDTA (Gibco), centrifuged at $1000 \mathrm{rpm}$ for 5 minutes at $4^{\circ} \mathrm{C}$ and resuspended in serum-free high glucose DMEM at a concentration of 100000 cells $/ \mathrm{mL}$. Cells were kept on ice for no longer than 1 hour.

Animals were anesthesized with $2 \%$ isoflurane (Piramal). Their heads were shaved and aseptically prepared. Animals were then placed in a stereotaxic frame (Harvard Apparatus) and a midline incision was made along the scalp. A burr hole was made $\sim 0.2 \mathrm{~mm}$ posterior from Bregma, $2.0 \mathrm{~mm}$ lateral from the sagittal suture. A 26 gauge needle was inserted to $4 \mathrm{~mm}$ below the dura and $10 \mathrm{uL}$ of the cell suspension was injected into the brain over a 10 minute period using a micropump (UltraMicroPump, World Precision Instruments). The needle was left in place for an additional 3 minutes following completion of the injection, and then withdrawn at 1 $\mathrm{mm} / \mathrm{min}$. The burr hole was sealed with bone wax (Surgical Specialties) and the incision was closed with wound glue (Vetbond, 3M). Animals were given buprenorphine (DOSE, Buprenex) for the first 24 hours and antibiotic water (10 mg/kg, Baytril, Bayer) for 2 weeks following the inoculation. 


\section{DETERMINING MAXIMUM TOLERATED DOSE OF CDDP-BPN}

The published maximum tolerated dose (MTD) of systemically-administered CDDP in rats is $6 \mathrm{mg} / \mathrm{kg}^{317}$. To determine the MTD of CDDP-BPN when given in conjunction with MRgFUS, 4 healthy rats received $5 \mathrm{mg} / \mathrm{kg}$ systemically-administered CDDP-BPN, followed immediately by BBB disruption using the sonication protocol described below. All four animals perished within 24 hours of treatment. An additional 12 animals were divided into four groups: $2.5 \mathrm{mg} / \mathrm{kg}$ CDDP-BPN + MRgFUS, $0.5 \mathrm{mg} / \mathrm{kg}$ CDDP-BPN + MRgFUS, $0.1 \mathrm{mg} / \mathrm{kg}$ CDDP-BPN + MRgFUS and MRgFUS alone. All animals recovered from treatment and survived 7 days post-treatment. $2.5 \mathrm{mg} / \mathrm{kg}$ was determined to be the MTD of CDDP-BPN with concurrent MRgFUS treatment.

\section{BLOOD-TUMOR BARRIER DISRUPTION WITH MRGFUS}

On the day of treatment, animals were anesthetized with an intraperitoneal injection of Ketamine (40 mg/kg, Fort Dodge) and Dexdomitor $(0.2 \mathrm{mg} / \mathrm{kg}$, Pfizer) in sterilized water. A tail vein catheter was inserted to allow intravenous (i.v.) delivery of microbubbles, CDDP-BPN, unecapsulated CDDP, and contrast agent. Animal skulls were depilated and positioned in a degassed water bath coupled to the FUS system. Rats receiving FUS were given a coinjection of therapeutic $(2.5 \mathrm{mg} / \mathrm{kg})$ and MBs (1E5 $\mathrm{MBs} / \mathrm{g}$ body weight) immediately prior to sonication.

All sonications were performed using a $1.14 \mathrm{MHz}$ single element focused transducer (FUS Instruments, Toronto, CA) operating at a $0.5 \%$ duty cycle for 2 minutes. Non-derated peak negative pressure was either 0.6 MPa or 0.8 MPa. Prior to placing the animal on the MRI table, the FUS system was passively aligned with a 3T MRI system (Trio, Siemens) using an anechoic Zerdine phantom (CIRS, Inc, Norfolk, VA). After the animal was coupled to the FUS system, high resolution contrast enhanced $(0.5 \mu \mathrm{l} / \mathrm{g}$ body weight, Magnevist, Bayer), T1-weighted scans were performed and utilized to locate the tumor boundaries with high accuracy. A 9-spot square grid, approximately $3 \mathrm{~mm}$ by $3 \mathrm{~mm}$ laterally and $5 \mathrm{~mm}$ deep, was centered over the tumor. 5 locations were sonicated within one 2 min sonication period using interleaved sonication mode, and the remaining 4 targets were treated with an additional 2 min sonication. Sonications were spaced 6 minutes apart to allow clearance of the first dose of MBs. An additional dose of MBs was injected immediately prior to the second sonication. Immediately following the final sonication, MRI contrast agent $(0.25 \mu \mathrm{l} / \mathrm{g}$ body weight) was delivered intravenously and the high resolution contrast enhanced T1-weigted scan was repeated to verify BTB/BBB disruption. Animals were then removed from the MRI table, given Antisedan ( $2 \mathrm{mg} / \mathrm{kg}$, Zoetis) in $5 \mathrm{~mL}$ warm saline subcutaneously, and placed on a heating pad at $37^{\circ} \mathrm{C}$ for 30 minutes. 
Contrast enhanced T1-weighted images were taken weekly prior to each treatment. Enhancing tumor diameter was calculated from thresholded images taken sagittally through the tumor at its maximum diameter. Enhancing tumor volume was calculated using the formula $\mathrm{V}=\frac{4}{3} \pi \mathrm{r}^{3}$. Percent tumor growth inhibition was calculated as $\% \mathrm{TGI}=100 \times \frac{\mathrm{V}_{\mathrm{c}-} \mathrm{V}_{\mathrm{t}}}{\mathrm{V}_{\mathrm{c}}-\mathrm{V}_{\mathrm{o}}}$ where $\mathrm{V}_{\mathrm{c}}$ and $\mathrm{V}_{\mathrm{t}}$ are the median tumor volumes in the treated groups and control group, respectively, on day 28 and $V_{o}$ is the median tumor volume at Day 14. Significance was determined using a two-way ANOVA.

\section{PROCESSING AND IMAGING OF SONICATED TISSUE}

Following euthanasia, animals' brains were excised for histology. In animals that received fluorescently labelled CDDP-BPN or PS-PEGs, the left and right carotid arteries were cannulated and each artery was perfused with $22 \mathrm{~mL} 2 \%$ heparinized saline prior to brain excision. Brains were dessicated in a $30 \%$ sucrose solution at $4{ }^{\circ} \mathrm{C}$ for 24 hours or until brains sunk. Dessicated brains were placed in O.C.T. compound (Sakura Finetek, Torrance, CA) for one hour at $21^{\circ} \mathrm{C}$ before freezing at $-80^{\circ} \mathrm{C}$. Transverse $8 \mu \mathrm{m}$ cryosections were mounted, stained for H\&E or endothelial cells (1:200 Lectin 488 for 1.5 hours at $21^{\circ} \mathrm{C}$, Invitrogen), and sealed with Prolong Gold (Invitrogen, Grand Island, NY) for imaging. H\&E sections were imaged on a Nikon TE300 confocal microscope equipped with a 20x oil objective, while nanoparticle delivery analysis on Lectin-stained sections was performed on a Nikon TE300 confocal microscope equipped with a 20x oil objective. An Optronics QuantiFIREXI camera was used to capture the images for analysis.

Confocal images were analyzed in MATLAB using a code developed in our lab. The nanoparticle (red channel) and endothelial (green channel) signals were separated for processing. Each channel was thresholded and converted to black and white using Otsu's method. Total nanoparticle coverage and vascular density were calculated as the total number of pixels above threshold in the red and green channels, respectively, normalized by the total number of pixels and expressed as a percentage.

\section{RESULTS}

\section{PHYSICOCHEMICAL CHARACTERIZATION OF CDDP NANOPARTICLES}

We encapsulated CDDP in conventional un-PEGylated nanoparticles (CDDPUPN) and in highly PEGylated nanoparticles (CDDP-BPN), each with high CDDP loading efficiency and diameters smaller than the ECM pore size within the brain 169 (Table 2.1). CDDP-BPN possessed a more uniform size distribution compared to the CDDP-UPN (polydispersity index of $0.15 \pm 0.01$ and $0.2 \pm 0.03$, respectively). The PEG coating on the CDDP-BPN shielded the anionic carboxyl side groups of the PAA, thereby reducing the surface charge from $-35.2 \pm 0.45 \mathrm{mV}$ for CDDP-UPN to near 
neutral $(-3.27 \pm 0.48 \mathrm{mV})$ for CDDP-BPN. The BPN formulations encapsulated nearly twice the amount ( $40 \pm 5 \% \mathrm{w} / \mathrm{w})$ of CDDP as the UPN formulation $(20 \pm 2 \% \mathrm{w} / \mathrm{w})$. Both NP formulations were spherical in shape (Fig2.1A). The hydrodynamic diameter of a CDDP-BPN suspension was stable for up to 24 hours in both serum and ACSF, but CDDP-UPN showed a rapid increase in hydrodynamic diameter after 6 hours in ACSF, indicating particle aggregation (Fig2.1B). Approximately $\sim 30 \%$ of total CDDP was release from CDDP-BPN over a 7 day period (Fig2.1C).

Table 2.1. Physiochemical properties of CDDP-loaded nanoparticles.

\begin{tabular}{|c|c|c|c|c|}
\hline $\begin{array}{c}\text { Nanoparticle } \\
\text { type }\end{array}$ & $\begin{array}{c}\text { Hydrodynamic } \\
\text { Diameter } \\
\pm \text { SEM (nm) })^{\dagger}\end{array}$ & $\begin{array}{c}\zeta \text {-potential } \\
\pm \text { SEM (mV) }\end{array}$ & $\begin{array}{c}\text { Polydispersity } \\
\text { index (PDI) } \\
\pm \text { SEM }^{\dagger}\end{array}$ & $\begin{array}{c}\text { Cisplatin Loading density } \\
\pm \text { SEM (\% w/w) }\end{array}$ \\
\hline CDDP-UPN & $65.0 \pm 5.1$ & $-35.2 \pm 0.45$ & $0.2 \pm 0.03$ & $20 \pm 2$ \\
\hline CDDP-BPN & $45.3 \pm 2.5$ & $-3.27 \pm 0.48$ & $0.15 \pm 0.01$ & $40 \pm 5$ \\
\hline
\end{tabular}

${ }^{\dagger}$ Hydrodynamic diameters and PDI were measured in $10 \mathrm{mM} \mathrm{NaCl}$ at $\mathrm{pH}$ 7.0. Data represent the mean $\pm \operatorname{SEM}(\mathrm{N} \geq 3$ measurements).

$\ddagger \zeta$-potentials were measured by laser Doppler anemometry in $10 \mathrm{mM} \mathrm{NaCl}$ at pH 7.0. Data represent the mean \pm SEM ( $N \geq 3$ measurements).
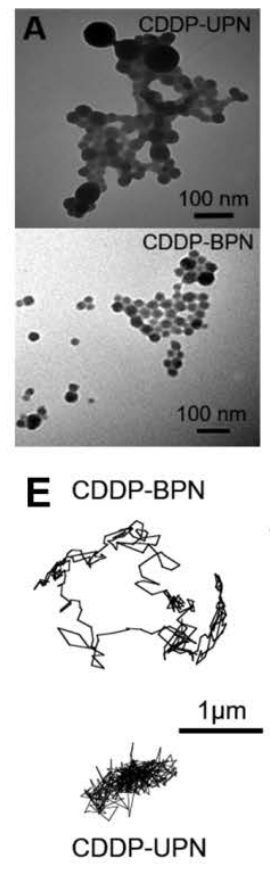

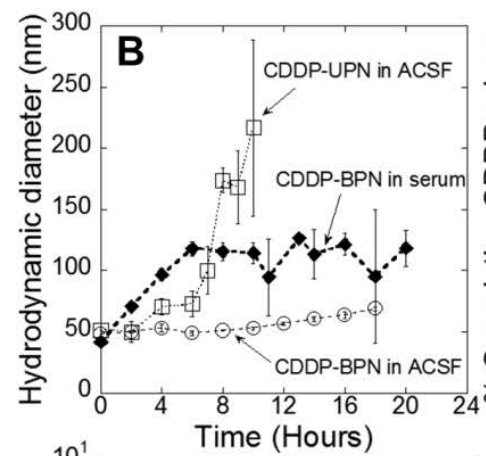

${ }^{10^{1}}$

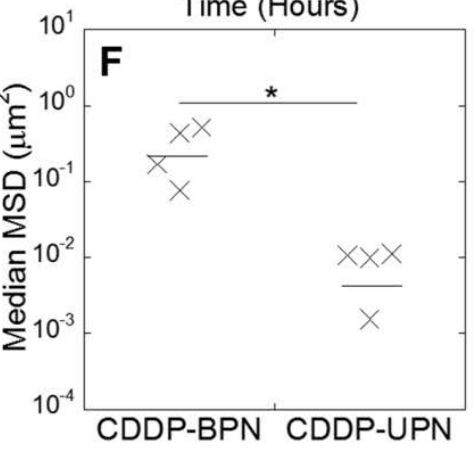

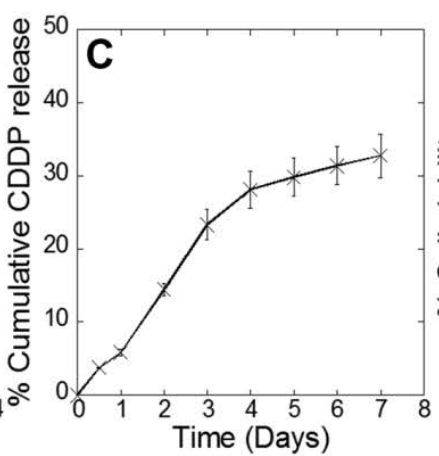
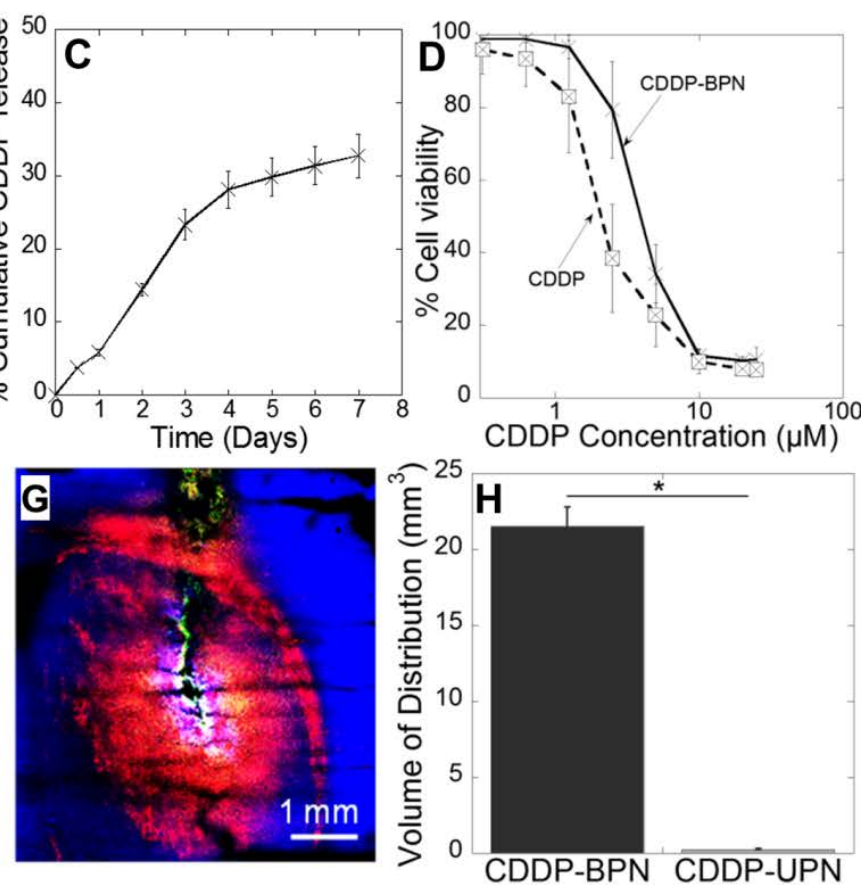

Figure 2.1: Physiochemical properties and diffusion behaviors of CDDP-loaded nanoparticles. (A) Transmission electron micrograph of CDDP-BPN (upper panel) and CDDP-UPN (lower panel). Scale bars $=100 \mathrm{~nm}$. (B) Stability of CDDP-BPN and CDDP-UPN in ACSF and media with 10\% FBS over time. (C) In vitro drug release kinetics of CDDP-BPN in PBS ( $\mathrm{pH} \mathrm{7.0)}$ at $37^{\circ} \mathrm{C}$. (D) In vitro cytotoxicity of carrier-free CDDP and CDDP-BPN in F98 rat GBM cells. (E) Representative trajectories of CDDP-BPN and CDDPUPN diffusing in rat brain tissue ex vivo. (F) Median MSD of CDDP-BPN and CDDP-UPN at a timescale of $t=1 \mathrm{~s}$ in at least $\mathrm{n}=3$ rat brain tissues ( $>100$ particles tracked in individual samples). ${ }^{*} \mathrm{P}<0.05$. (G) In vivo distribution of CDDP-BPN (red) and CDDP-UPN (green) in rat brain striatum following administration via CED. Cell nuclei are stained by DAPI (blue). Scale bar $=1 \mathrm{~mm}$. $(\mathrm{H})$ Volume of in vivo distribution of CDDP-BPN and CDDP-UPN. 
IN VITRO CYTOTOXICITY OF CDDP-BPN AGAINST RODENT GLIOMA CELLS

To determine whether BPN encapsulation of CDDP would alter the drug's potency, we treated F98 glioma cells with unencapsulated free CDDP (CDDP) and CDDP-BPN. Treatment with $10 \mu \mathrm{M}$ or more of CDDP, either free or encapsulated in BPN, reduced cell viability to less than 10\% (Fig2.1D). In agreement with our previous work ${ }^{316}$, there was no significant difference in the antitumor efficacy of CDDP compared to CDDP-BPN, leading us to conclude that the BPN formulation did not reduce inherent cytotoxicity of the drug.

\section{DENSE PEGYLATION ENHANCES CDDP NANOPARTICLE SPREADING} IN HEALTHY BRAIN TISSUE

Mean squared particle displacements (MSD) of both CDDP-UPN and CDDPBPN were calculated using multiple particle tracking (MPT) following NP administration in fresh rat brain slices ex vivo. Representative CDDP-BPN particle trajectories over 20 $s$ demonstrated unhindered diffusion compared to the CDDP-UPN, which were largely immobilized (Fig2.1E). At a timescale of $\mathrm{T}=1 \mathrm{~s}$, the mean MSD of the CDDP-BPN was 160-fold greater than their un-PEGylated controls, indicating that the dense PEG coat reduced particle interactions with the ECM and permitted particle spreading over time (Fig2.1F).

\section{DENSE PEGYLATION ENHANCES IN-VIVO DISTRIBUTION OF CDDP NANOPARTICLES FOLLOWING INTRACRANIAL ADMINISTRATION}

To determine whether the increased NP diffusion rate ex vivo would translate into greater volume of distribution following local NP administration in vivo, we coadministered fluorescently labeled CDDP-BPN and CDDP-UPN into the rat striatum via CED and evaluated the volume of distribution $(\mathrm{Vd})$ for each particle formulation (Fig2.1G). CDDP-BPN distribution was homogeneous, producing a $\mathrm{Vd}$ of $21.5 \pm 1.25$ $\mathrm{mm}^{3}$, compared to only $0.25 \pm 0.025 \mathrm{~mm}^{3}$ for the CDDP-UPN formulation, a 100-fold difference $(\mathrm{Fig} 2.1 \mathrm{H})$. Note that CDDP-UPN were used as a control for CDDP-BPN for nanoparticle characterization, but they were not tested in combination with MRgFUS targeted delivery due to their inability to widely distribute throughout the rat brain tissue following local administration. 


\section{VERIFYING BLOOD-TUMOR BARRIER DISRUPTION}

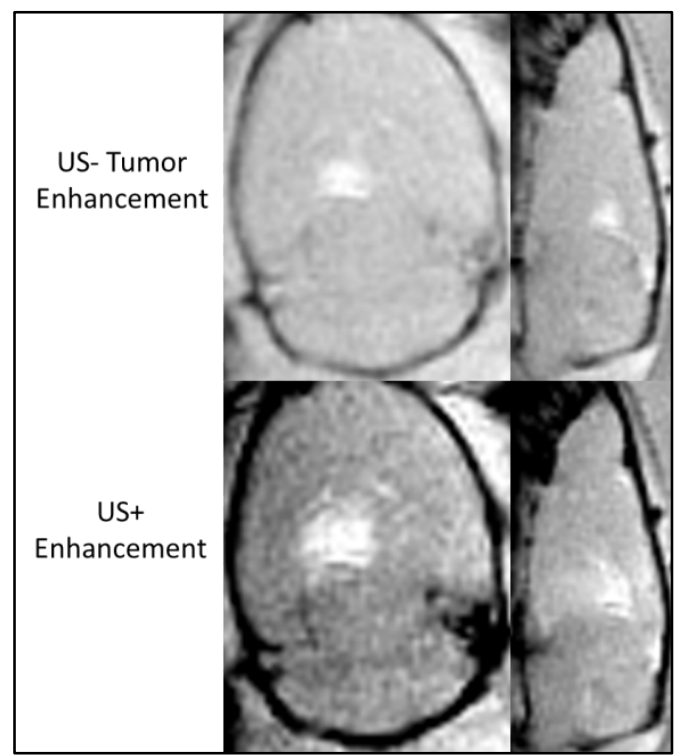

\begin{tabular}{l} 
Figure 2.2: \\
\hline MRgFUS \\
delivers \\
gadolinium \\
across the \\
blood-tumor and \\
blood-brain \\
barriers. Contrast \\
enhanced T1- \\
weighted MRI \\
images \\
demonstrate FUS \\
treatment of a \\
tumor in a rat \\
model of \\
glioblastoma \\
multiforme.
\end{tabular}

To achieve accurate targeting of the tumor with MRgFUS, tumors must be developed enough to have vasculature that is permeable to an MR contrast agent (Magnevist $₫, \mathrm{MW}=938$ ). Early stage tumors appear as small (1-3mm diameter) areas of focal enhancement prior to FUS treatment (Fig2.2, US-). A 9spot, $3 \mathrm{~mm} \times 3 \mathrm{~mm} \times 5 \mathrm{~mm}$ grid is sonicated centered over the tumor, producing enhanced vascular permeability within the tumor and in the normal brain tissue immediately surrounding the tissue, as demonstrated by post-FUS contrast enhanced MR images (Fig2.2, US+).

\section{BPN DISTRIBUTION IN THE BRAIN FOLLOWING MRGFUS}

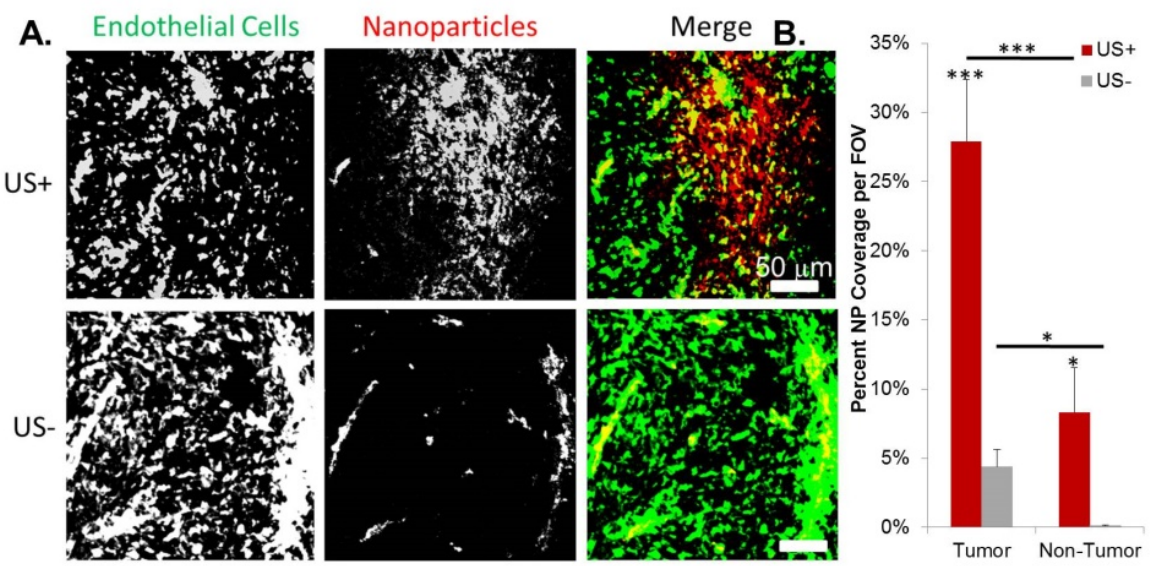

Figure 2.3: Focused ultrasound increases delivery of brainpenetrating nanoparticles across the BTB and BBB in the $9 \mathrm{~L}$ glioma model. Confocal microscopy images of rat brain slices demonstrate a significant $(p=0.000) 6.4$ fold increase in NP delivery in tumors treated with FUS $(A, B)$ compared to untreated tumors $(A, B)$. Ultrasound treatment also produced a significant 61.1 fold increase in NP delivery in normal tissue immediately beyond the tumor border (B). $n=6$.
To evaluate BPN delivery across the BTB and $\mathrm{BBB}$, two intracranial glioma models were used: 9L and F98. Confocal microscopy images were used to evaluate BPN delivery in sonicated and unsonicated tumors 24 hours after treatment. In the 9L model of glioma, BPN delivery within MRgFUS treated (US+) tumors was more homogeneous (Fig2.3A). In untreated (US-) tumors, BPN

deposition occurred primarily within large, abnormal vessel structures (Fig2.3A). Average NP coverage per field of view was $28 \%$ in US+ tumors, compared to $4 \%$ in untreated tumors (Fig2.3B). This 6.4-fold increase was highly significant $(p<0.001)$. In 
tissue with an intact BBB just beyond the tumor border ("Non-Tumor"), MRgFUS treatment produced a significant 61.1-fold increase in percent NP coverage per field of view, from $0.1 \%$ in untreated tissue to $8.3 \%$ in US+ tissue. In both treated and untreated animals, there was a significant increase in BPN delivery in the tumor compared to nontumor tissue. Additionally, there was a significant interaction effect $(p=0.01)$ between tissue type (tumor vs. non-tumor) and treatment (US+ vs. US-).

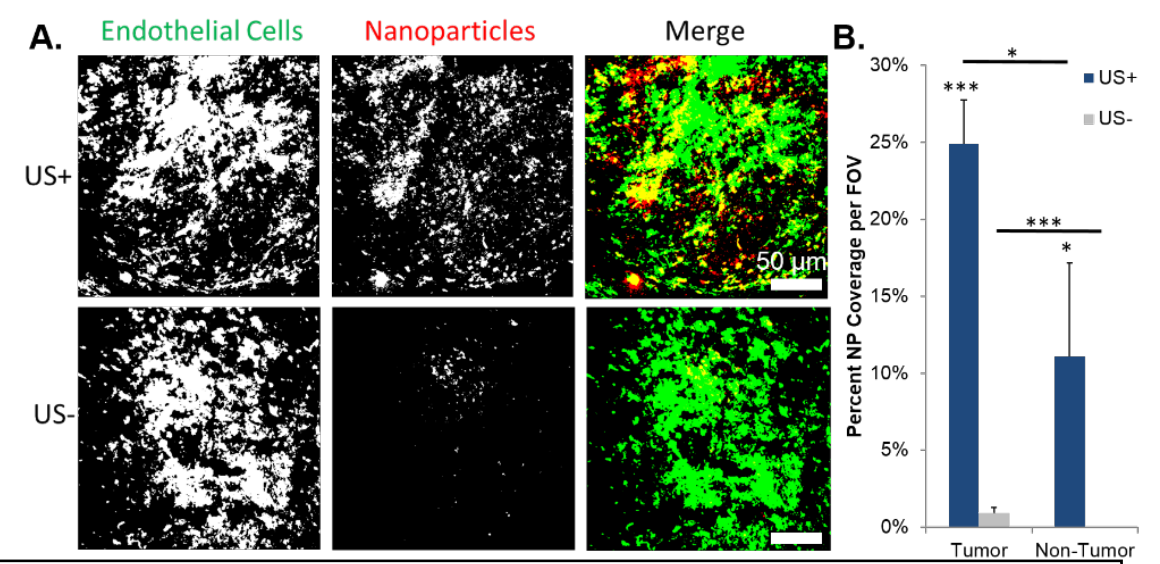

Figure 2.4: Focused ultrasound increases delivery of brainpenetrating nanoparticles across the BTB and BBB in the F98 glioma model. Confocal microscopy images of rat brain slices demonstrate a significant $(p=0.000) 27.6$ fold increase in BPN delivery in tumors treated with FUS $(A, B)$ compared to untreated tumors (A, B). Ultrasound treatment also produced a significant increase in BPN delivery in normal tissue immediately beyond the tumor border (B). $n=4$.
BPN delivery was similar in the F98 model of glioma. MRgFUS treatment improved the delivery and distribution of BPNs (Fig2.4A). In untreated (US-) tumors, BPNs remained contained within large, abnormal vessel structures (Fig2.4A) Average NP coverage per field of view was $25 \%$ in US+ tumors, compared to $1 \%$ in untreated tumors (Fig2.4B). This increase was highly significant $(p=0.000)$. In the region

beyond the tumor border with normal vasculature ("Non-Tumor"), no BPNs were observed in untreated animals, while animals treated with MRgFUS averaged $11 \% \mathrm{NP}$ coverage per FOV $(p=0.01)$. In both treated and untreated animals, there was a significant increase in BPN delivery in the tumor compared to non-tumor tissue. Additionally, there was a significant interaction effect $(p=0.02)$ between tissue type (tumor vs. non-tumor) and treatment (US+ vs. US-). Further experiments in the F98 model using fluorescently labelled CDDP-BPNs demonstrated similar results, with MRgFUS producing a 30 -fold increase $(p<0.001)$ in NP coverage in tumor tissue and a 75 -fold increase $(p=0.005)$ in non-tumor tissue (data not shown). 
To evaluate the hypothesis that heterogeneous vascular density affects BPN delivery across the BTB, we examined the relationship between percent vascular area (\%VA) and BPN delivery within treated and untreated tumors. MRgFUS-mediated BPN delivery across the BTB varied significantly with \%VA in the $9 \mathrm{~L}$ model $(p=0.005)$, Fig2.5. Maximum NP coverage occurred in tumor regions with 50-60\% VA and 60-70\% VA in the F98 and 9L models, respectively, and was at least 3 fold higher than in less vascularized regions $(<40 \% \mathrm{VA})$ and at least 1.5 fold higher than in highly vascularized regions (>80\% VA). In untreated animals, BPN delivery was not affected by \%VA and was similar to poorly perfused ( $<30 \% \mathrm{VA})$ regions in US+ tumors (data not shown).

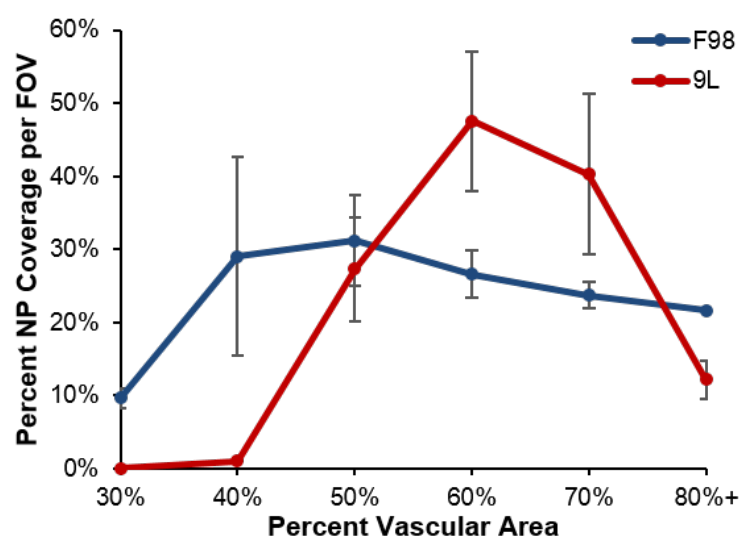

Figure 2.5: Focused ultrasound-mediated delivery of brain-penetrating nanoparticles across the BTB is dependent on vascularity. In both $9 \mathrm{~L}$ and F98 gliomas, FUS-mediated delivery of BPNs varied with the tumors' percent vascular area (VA). In the 9L model, optimal BPN delivery in MRgFUS treated animals occurred in tumor regions with $60-70 \%$ VA, while BPN delivery in the F98 model was highest in tumor regions with $50-60 \%$ VA . $n \geq 4$.

\section{TUMOR GROWTH CONTROL}

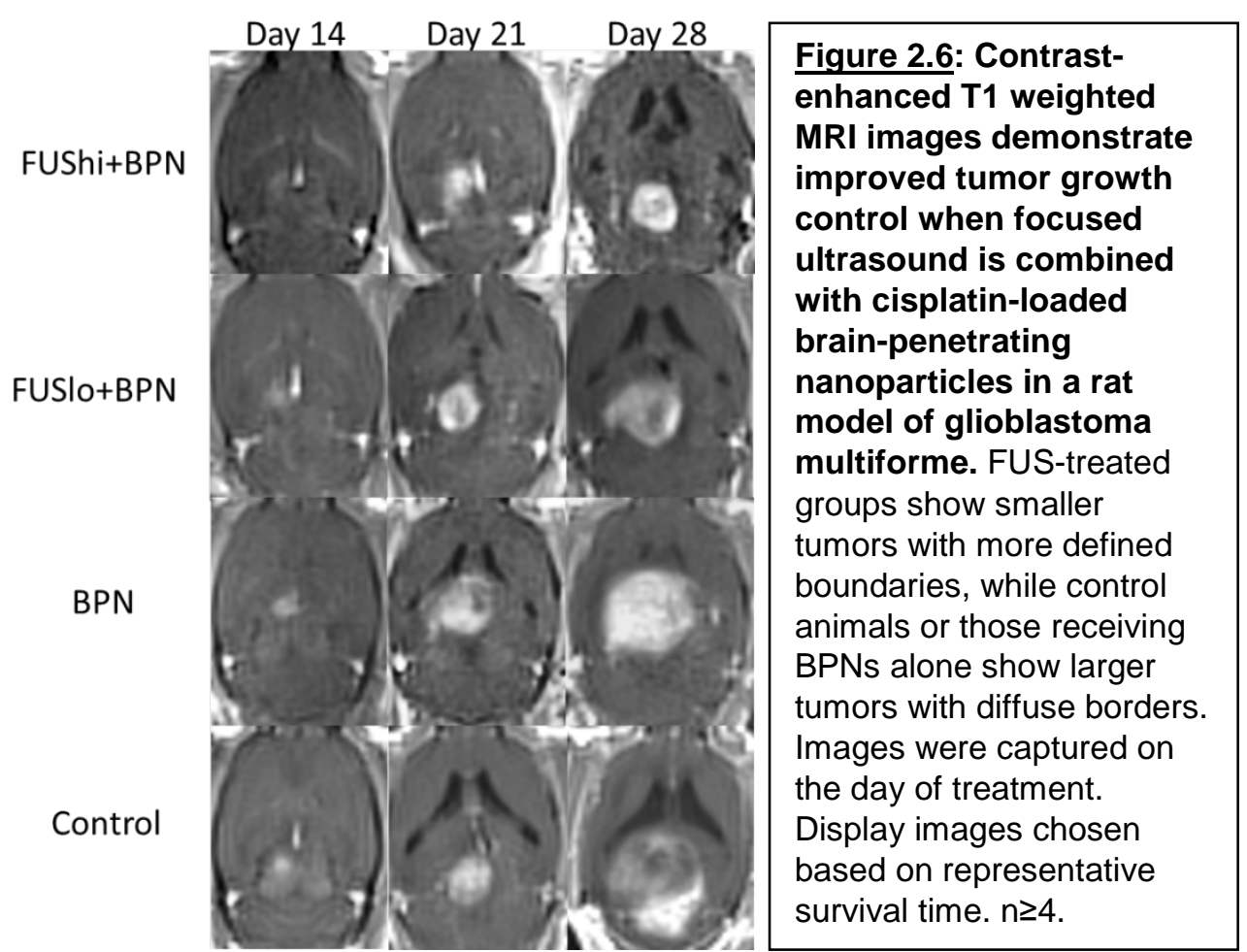

To evaluate tumor growth control, contrast enhanced T1 weighted MR images were used to determine the enhancing tumor volume in each animal. Note that in this section, BPN refers to CDDP-loaded BPN. Measurements were made weekly, beginning when the tumor was 
first visible on MRI (day 14). Enhancing tumor volume was identical across all groups at the start of treatment (day 14, Fig2.6,2.7A). Untreated control animals and animals receiving BPNs alone had tumors with diffuse borders (Fig2.6), typical of an invasive GBM tumor ${ }^{297}$, that grew rapidly between days 21 and 28 (Fig2.7A). Animals treated with 0.8 MPa FUS in combination with BPN had smaller tumors with more defined borders (Fig2.6), and demonstrated delayed tumor growth between days 21 and 28 (Fig2.7A). 0.8 MPa FUS+BPN (FUShi+BPN) produced the greatest tumor growth inhibition (61\% TGI, compared to untreated control) at day 28 (Fig 2.7B), a 15\% improvement over 0.6MPa FUS+BPN (FUSlo+BPN) and a 64\% improvement compared to BPN alone.

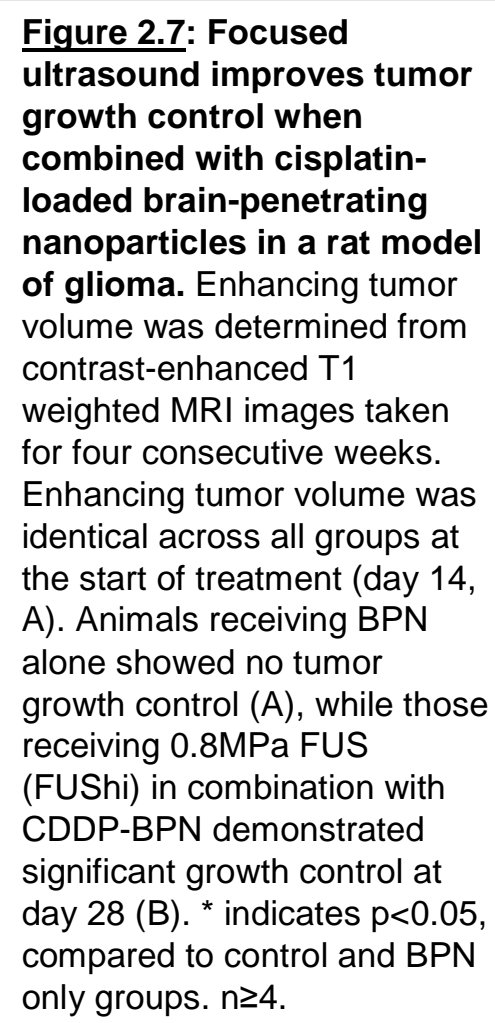

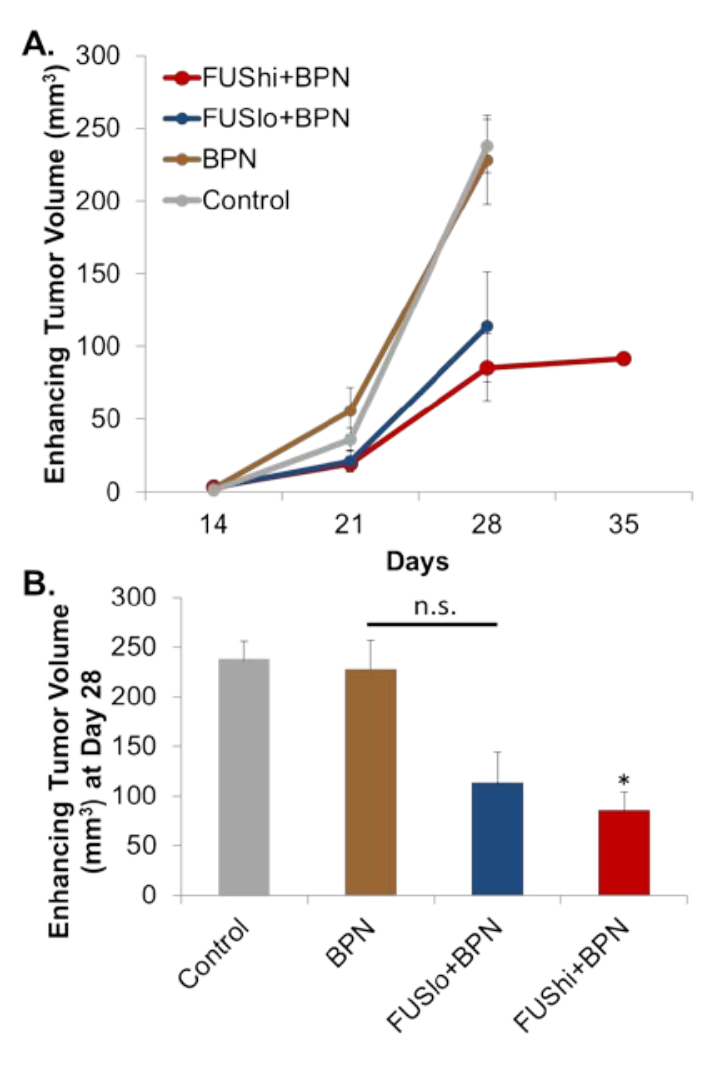


To evaluate the effects of FUS+ BPN, we generated Kaplan-Meier survival curves. $0.8 \mathrm{MPa}$ FUS in combination with BPN (FUShi+BPN) significantly extended survival (Fig 2.8) compared to untreated controls (maximum survival time of 35 and 28 days post inoculation, respectively). Maximum survival time was identical (32 days) for animals receiving 0.6 MPa FUS in combination with BPNs (FUSlo+BPN) or BPNs alone, but did not represent a significant improvement over untreated controls. Survival was analyzed using pairwise comparisons in a life table analysis with the Wilcoxon statistic.

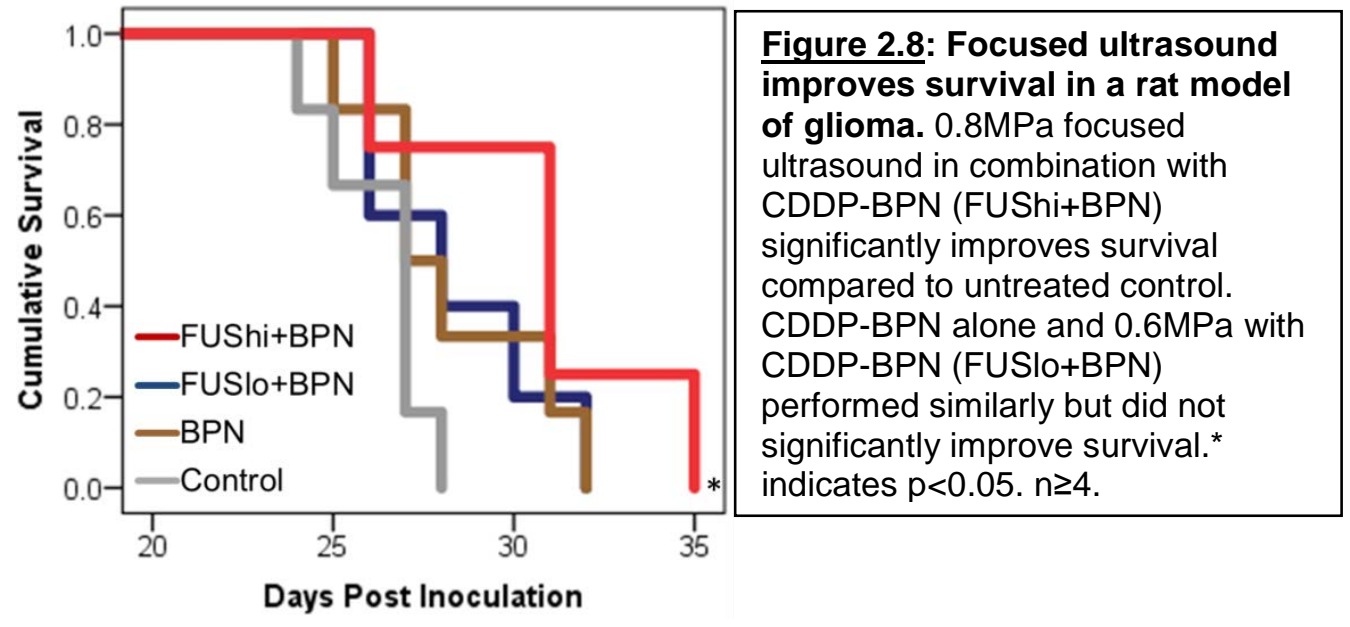

STATISTICAL ANALYSIS

Data in Figure 2.3b, 2.4B and 2.7 were analyzed using a two-way ANOVA. In data sets that showed a statistically significant interaction effect, pairwise comparisons and an analysis of simple main effects was conducted. In data sets that did not show a statistically significant interaction effect, pairwise comparisons and an analysis of main effects was performed. Data in Figure 2.5 were analyzed using a one-way ANOVA. Data in Figure 2.8 were analyzed using Kaplan-Meier survival analysis, followed by a Mantel-Cox log rank pairwise comparison. Mantel-Cox log rank pairwise comparisons were also performed for data in Figure 2.3B. All analyses were performed with IBM SPSS Statistics (Windows Version 22.0). Significance was set at $p<0.05$.

\section{DISCUSSION AND CONCLUSIONS}

Current treatment strategies for glioma are unable to effectively control tumor recurrence, the leading cause of death for patients ${ }^{220}$. Invasive tumor cells that lie beyond the enhancing tumor border in areas protected by the intact BBB limit the efficacy of systemically administered drugs $19,306,307,318$, and locally administered therapeutics do not diffuse well beyond the resection cavity ${ }^{165,278}$. Standard polymeric nanoparticles can provide extended drug release; however, they are immobilized in the complex tumor parenchyma ${ }^{319,320}$ and do not spread well within tumors. Here, we evaluated the potential of a novel combination of MRgFUS and CDDP-loaded brain 
penetrating nanoparticles in the treatment of GBM. We designed a polymeric nanoparticle formulation for enhanced delivery and distribution within the brain 165,169,178 following delivery across the BBB/BTB with MRgFUS ${ }^{177}$. These densely PEGylated BPN were shown to deliver higher concentrations of CDDP in a sustained manner to a larger population of tumor cells. Importantly, MRgFUS-mediated BBB/BTB disruption enhanced both the delivery and distribution of CDDP-BPN, improving efficacy against both the tumor bulk and infiltrating tumor cells. We conclude that this approach may provide improved therapeutic outcomes and potentially decreased rates of glioma recurrence.

As a platinum-based chemotherapeutic, CDDP is a highly potent and nonspecific inhibitor of DNA synthesis and repair ${ }^{317}$. The clinical use of CDDP in gliomas has been limited by significant neurotoxicities ${ }^{300,301}$ which mandated the use of low CDDP doses and invasive local delivery methods ${ }^{303,321}$. These difficulties lead to the development of less toxic but less effective compounds ${ }^{322,323}$. Sustained, local release of low concentrations of CDDP can both increase the effectiveness of therapy and decrease off-target toxicity ${ }^{316,321,324-327}$. The particles developed here show sustained CDDP release over 7 days and are highly effective against F98 glioma cells in vitro. Combined with weekly MRgFUS, these CDDP-BPN could provide stable local drug concentrations with reduced systemic and local toxicities.

As we have previously reported ${ }^{169,177,178,316}$, a dense coating of PEG reduces the particle polydispersity and the net charge at the particles' surface, producing a monodisperse, near-neutrally charged BPN that is smaller than the average pore size within the ECS of the brain parenchyma ${ }^{169}$. The combination of small particle size and non-adhesive surfaces significantly increased the rate of spreading of the particles in healthy brain tissue, as measured by MSD and Vd, in agreement with previous studies with other BPN formulations ${ }^{169,178,316}$. The use of activated, aquated cisplatin during BPN formulation doubled drug loading efficiency and significantly extended drug release compared to our previous formulation using un-activated CDDP ${ }^{316}$. Additionally, these CDDP-BPN remain stable in both blood and ACSF for up to 24 hours, making them ideal candidates for MRgFUS-mediated delivery, as they will continue to circulate in the blood, accumulate, and spread within brain or tumor tissue following BBB/BTB disruption.

Local administration of chemotherapeutics has the potential to deliver high concentrations of therapeutics in the tumor and has achieved clinical success as demonstrated by the Gliadel $\circledast$ wafer, but limited diffusion of the drug from the delivery site has minimized its therapeutic efficacy $110,112,113$. Furthermore, local administration techniques are highly invasive and are associated with significant risks 220,328, particularly for therapeutics that require multiple treatments. MRgFUS is the only treatment modality capable of producing non-invasive, localized, reversible BBB disruption $47,281,311$ and has been shown to increase the delivery of therapeutics across the BTB as well ${ }^{61,101,309,329}$. In agreement with this body of work as well as our previous work ${ }^{177}$, we show here that MRgFUS significantly increases both the total delivery and 
distribution of BPNs within the tumor parenchyma and surrounding normal tissue in both the $9 \mathrm{~L}$ and F98 glioma models. The enhanced permeation and retention (EPR) effect produced very little BPN delivery in either tumor, emphasizing the need for a delivery mechanism. The low efficiency of EPR-mediated delivery is further demonstrated by the poor performance of the CDDP-BPN group in the drug delivery arm of the experiment, whose tumors were of similar size and morphology to untreated controls. FUS-mediated BTB disruption increases pinocytotic vesicle formation and upregulates caveoliassociated proteins ${ }^{61}$. Additionally, tumor permeability, as measured by gadolinium enhancement, is enhanced following FUS treatment ${ }^{101}$. These mechanisms may lead to increased BPN delivery and uptake within the tumor.

While MRgFUS-mediated delivery produced more homogeneous BPN distribution throughout the tumor, BPN delivery varied with vascular density in both tumor models. This spatial variation may reflect changes in the vasculature itself, as it has been shown that FUS-induced BBBD is dependent on vessel size, and vessels over $30 \mu \mathrm{m}$ in diameter do not demonstrate BBBD following FUS ${ }^{2,63}$. Furthermore, areas with high vascular density may have higher interstitial pressures ${ }^{330}$ and reduced extracellular space available, thus reducing BPN uptake. Diffusion is known to vary throughout gliomas ${ }^{331}$. Optimal BPN delivery occurred at slightly different vascular densities in the two models, and the degree of dependence of BPN delivery on vascular density was different as well. This likely reflects inherent differences in the characteristics of the tumors, as F98 gliomas are known to co-opt existing vasculature, while $9 \mathrm{~L}$ gliomas are highly angiogenic and significantly alter vascular networks ${ }^{314}$. This data may help determine optimal treatment schedules, as well as which patients may benefit most from MRgFUS.

Here, we demonstrate that three weekly treatments with $0.8 \mathrm{MPa}$ MRgFUS + CDDP-BPN significantly improved tumor growth control and animal survival. We previously determined ${ }^{177}$ that $0.6 \mathrm{MPa}$ was sufficient to produce significant enhancements in BPN delivery across the BBB in normal tissue, and we demonstrated here that $0.6 \mathrm{MPa}$ significantly increased BPN delivery across the BTB in both the $9 \mathrm{~L}$ and F98 glioma models. However, abnormal tumor vasculature ${ }^{314}$ and parenchyma ${ }^{332}$ combined with the aggressive nature of the tumor $312,313,332$ required the use of slightly higher pressure to achieve a treatment effect. In addition to reducing tumor volume, animals treated with MRgFUS + CDDP-BPN had more defined tumor boundaries. While MRgFUS + CDDP-BPN was not curative, it did control the growth and infiltration of the tumor, indicating that this may be a viable treatment option following surgical de-bulking.

Previous work has demonstrated that repeated treatments and higher FUS pressures produce improvements in tumor growth control and animal survival $3,12,309,333$. In the $9 \mathrm{~L}$ model, a single high-pressure treatment (0.8 MPa) of FUS with liposomal doxorubicin produced a significant $24 \%$ increase in median survival time, but was not curative ${ }^{3}$. A follow-up study in the same model utilized three weekly FUS + liposomal doxorubicin (again at $0.8 \mathrm{MPa}$ ) to achieve complete tumor regression in $3 / 8$ animals ${ }^{12}$. The doxorubicin liposomes are large $(\sim 100 \mathrm{~nm})$, and these studies did not determine 
whether FUS was able to deliver intact liposomes across the BBB/BTB. Given the FUS pressures used, it is likely that FUS disrupted the liposomes ${ }^{334}$ in the tumor vasculature and enhanced the delivery of the released doxorubicin across the BBB/BTB. FUS has been shown to increase delivery of free doxorubicin across the BTB in preclinical glioma models ${ }^{5,98}$. A similar study in the same glioma model showed that a single $0.75 \mathrm{MPa}$ FUS + temozolomide treatment increased survival by 3 days ${ }^{109}$. Treatment with FUS+CDDP-BPNs was similar to treatment with FUS+TMZ, but less effective than FUS+ liposomal doxorubicin. However, we were able to demonstrate delivery of intact $60 \mathrm{~nm}$ particles (much larger than either free doxorubicin or TMZ) across the BTB and are working in a much more infiltrative tumor model ${ }^{312}$.

To our knowledge, the only other study to attempt a treatment effect with FUS in the F98 glioma model utilized transfected cells expressing HSV1-tk, which makes them highly susceptible to the small-molecule antiviral drug ganciclovir. In this model, FUS+ganciclovir produced up to $80 \%$ tumor regression, compared to $5 \%$ for ganciclovir alone ${ }^{335}$. However, it should be noted that these transfected cells were several fold less aggressive than wild-type F98 cells, most likely due to increased immunogenicity. Rousseau et al showed impressive survival benefits with a single CED injection of $6 \mu \mathrm{g}$ of CDDP in the F98 glioma model, increasing median survival time by 7 days and producing a cure in $3 / 23$ animals ${ }^{336}$. An earlier study by the same group showed similar results with a $3 \mu \mathrm{g}$ dose of CDDP via CED, although no animals achieved a cure ${ }^{337}$. These CED studies imply that FUS+CDDP-BPN delivered less than $3 \mu \mathrm{g}$ of CDDP across the BTB, which would equate to $0.12 \%$ injected dose (\%ID). One study has demonstrated delivery of 2.3\%ID of a small molecule following FUS ${ }^{338}$, and given the much larger size of the CDDP-BPN, it is not surprising that the \%ID was so low. Ultimately, gliomas may prove too aggressive to control with non-invasive techniques, particularly if those techniques are dependent on systemic administration and are therefore limited by systemic toxicities. Therapies which reduce effects to tumor cells alone, such as gene or targeted therapies, may more amenable to combination with MRgFUS. Additionally, approaches which sensitize cancer cells to the therapeutic and thus reduce the required systemic dose may be more effective.

The translational applicability of this CDDP NP platform for treatment of tumors has been established by a similar, peptide-based CDDP NP which has demonstrated promising Phase I/II results and is currently under investigation in a Phase III clinical trial for pancreatic cancer ${ }^{339}$. In combination with MRgFUS, these BPNs can provide enhanced, sustained drug delivery to the tumor bulk and the surrounding tissue that would otherwise be protected by the BBB. This combination therapy provides the ability to non-invasively deliver chemotherapeutics in a targeted manner, reducing systemic toxicity and enabling repeated treatment. We anticipate that MRgFUS-mediated delivery of CDDP-BPN may be a promising treatment approach to prevent tumor recurrence in patients with high grade gliomas.

This is the first study demonstrating that MRgFUS enhances the delivery and efficacy of a polymeric nanoparticle in the treatment of glioma. MRgFUS has recently 
been awarded FDA approval for thermal tissue ablation in the brain, and there are ongoing clinical trials for MRgFUS in the treatment of glioblastoma multiforme. These trials, as well as those investigating the use of CDDP NP formulations in the treatment of cancer ${ }^{339}$, may be used as a precedent for translating this work to the clinic. This study highlights the benefits of such a combination therapy, particularly in gliomas, which are notoriously invasive and recur in over $90 \%$ of patients 220,295 . A non-invasive, repeatable drug delivery technique that limits systemic toxicity is a promising alternative to current therapies ${ }^{220}$. Furthermore, combining MRgFUS with biodegradable nanoparticle platforms permits the use of therapeutics which do not cross the BBB ${ }^{340}$ or are highly toxic ${ }^{13}$. BPN delivery with MRgFUS can improve antitumor efficacy by: (i) providing deeper brain tissue penetration of drug than is achieved with polymer implants (located only at the periphery in the surgical cavity), (ii) enabling multiple dosing regimens without the need for repeat surgery, (iii) facilitating local delivery for tumors with high surgical risk, and (iv) permitting patient-specific BPN design based on the unique tumor subtype and mutations. MR guidance adds the ability to identify and target specific regions, reducing off-target toxicity within the brain.

\section{FUTURE DIRECTIONS}

This work highlights the difficulty of designing a curative treatment for GBM ${ }^{220}$. While we demonstrated significant improvement in growth control and animal survival, the therapy did not produce tumor regression. One limitation of the work is the treatment timing. Due to a reliance on MR imaging for targeting, the first treatment did not occur until the tumor was visible on contrast-enhanced T1-weighted MR, indicating the tumor was well established and had compromised BBB function. Utilizing an alternative imaging method to verify tumor engraftment would permit initializing treatment earlier and may improve treatment outcome, particularly since it has been shown that MRgFUS produces greater improvements in BTB disruption in earlier stage tumors ${ }^{101}$. This may be due to increased abnormality of the vasculature as the tumor progresses ${ }^{314}$. Alternatively, slower-growing glioma models ${ }^{313}$ may provide a larger therapeutic window.

CDDP, while highly potent, is also highly toxic ${ }^{317}$. Systemic administration, while more palatable for clinical translation, reduces the maximum tolerated dose of these types of drugs ${ }^{324}$, even when they are encapsulated. MRgFUS increases local delivery, thus reducing the systemic dose, but additional methods could be used to further reduce systemic toxicities and permit higher local drug concentrations. Convectionenhanced delivery (CED) has been used to inject therapeutics directly into the brain $320,336,341$, but requires an invasive procedure. Alternatives include carotid artery injection or intranasal delivery. Carotid artery injection, rather than peripheral vein administration, provides more direct access to the vasculature within the brain without requiring access through the skull and can increase the percent injected dose that is delivered to the brain ${ }^{342}$. This may be particularly beneficial for fast-acting therapeutics or those with short systemic half-lives. Intranasal delivery offers a unique method to bypass the BBB, 
delivering drugs directly into the CSF via the olfactory pathway ${ }^{38,39}$. However, drug delivery and distribution is non-specific. Recently, MRgFUS has been used to improve the delivery and distribution of intranasally-administered drugs ${ }^{343,344}$, representing a new non-invasive treatment strategy.

In addition to altering the delivery strategy to reduce systemic toxicity, MRgFUS can be combined with less toxic or more targeted therapeutics. Repeated MRgFUS treatments with liposomal doxorubicin have been effective in a rat model of glioma ${ }^{5,12}$ and delivery of other agents, including TMZ ${ }^{109}$ and Herceptin ${ }^{129}$, have shown benefit as well. Gene delivery is also promising, as it offers the benefit of both highly targeted delivery and long-term effects ${ }^{345}$. Animal studies have demonstrated significant improvements in gene expression in the brain when gene vectors are combined with MRgFUS ${ }^{270}$. Additionally, immunotherapy is a promising new approach to the treatment of GBM, and MRgFUS offers unique benefits ${ }^{346}$. MRgFUS can deliver immunotherapeutics ${ }^{138}$ or immune cells ${ }^{84,266}$ across the BBB/BTB, and may act as a stand-alone immune adjuvant ${ }^{346}$. 
To determine whether the activation of microbubbles with ultrasound can enhance the adaptive antitumor immune response.

Unfocused ultrasound (US) can produce improvements in tumor growth control, but the mechanism is unclear. US-mediated improvement in T cell infiltration may greatly improve the efficacy of T-cell based therapies, such as anti-PD-1 treatment, and a better understanding of US-induced immunomodulation may allow us to utilize this technique with greater efficacy and predict how it will interact with other therapies. Immune-compromised mice will be used to distinguish the effects of $B$ and T cells in treated tumors. Pharmaceutical blockade of T cell trafficking with FTY720 and adoptive $T$ cell transfer with tagged $T$ cells will permit us to measure the contribution of $T$ cell trafficking and proliferation to ultrasound-mediated tumor growth control.

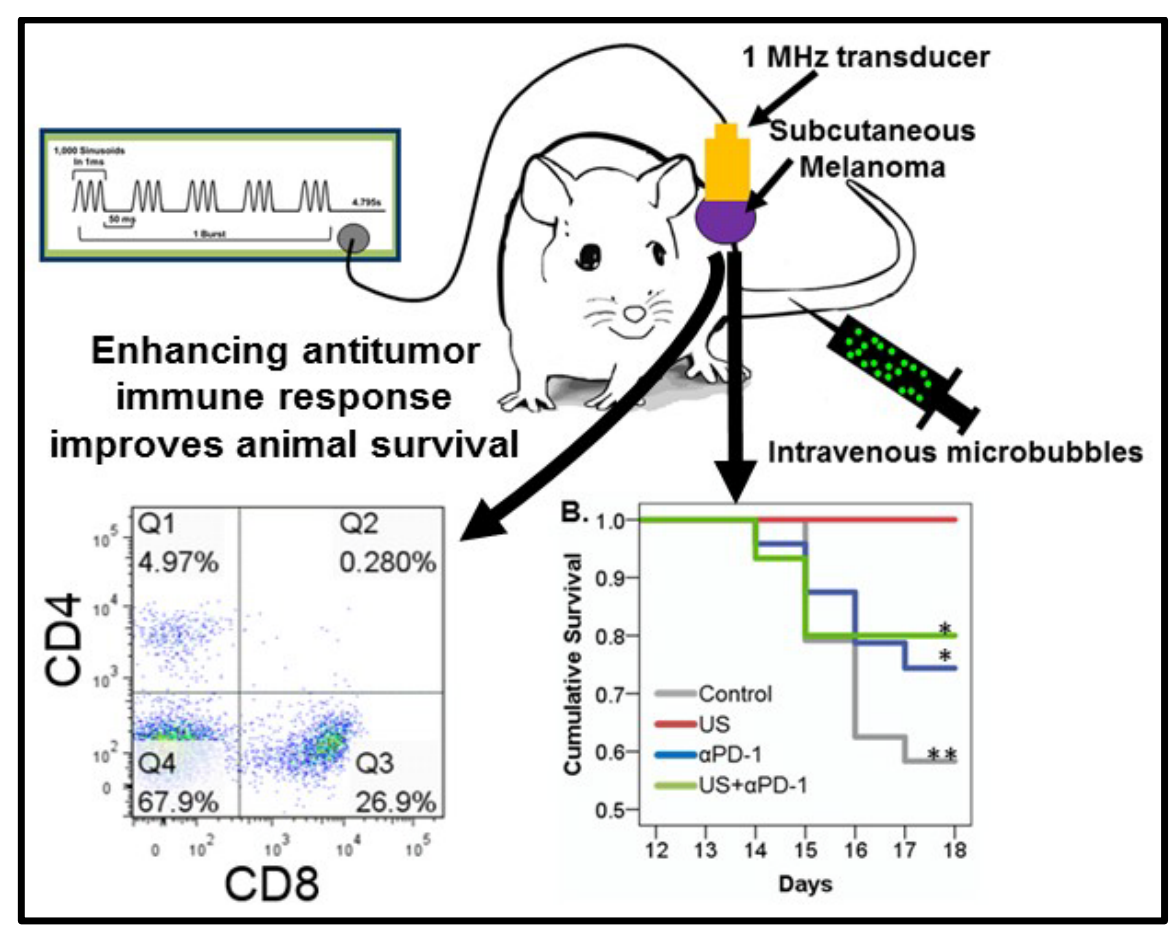


The incidence and mortality rates for melanoma have been increasing for the last 30 years at a pace faster than any other type of cancer ${ }^{347}$. While early stage melanoma is easily treated, metastatic melanoma is typically fatal, with 5 year survival rates between 5 and $10 \%{ }^{16,347}$. Until recently, first-line therapy consisted of aggressive chemotherapy, with response rates between $15-20 \%{ }^{232,347}$. One third to one half of responders experienced relapse or recurrence due to acquired drug resistance ${ }^{16,347}$. With the advent of immunotherapeutics, the way metastatic melanoma is treated has changed, but many challenges remain ${ }^{232}$.

While metastatic melanoma is highly immunogenic, tumors develop a host of mechanisms to avoid immune surveillance, including upregulation of inhibitory checkpoint ligands, increased production of immune-modulating cytokines and decreased antigen expression 196,211,347. Therapies that block, reverse or overcome these mechanisms can produce an aggressive, systemic antitumor immune response that has led to unprecedented improvements in patient survival 194,348. Many of these therapies act by altering the activity or density of tumor infiltrating immune cells. Antitumor immune responses are characterized by increases in tumor infiltrating natural killer cells (NK), dendritic cells (DC), and CD4+ and CD8+ T cells (CD4,CD8), while large regulatory $T$ cell (Treg) populations are associated with poor prognosis ${ }^{232,349-352}$. Macrophages can be either pro- or antitumorigenic, depending on their phenotype 209 . Furthermore, the ratios of these cell types, most notably the CD8/Treg ratio, can be a powerful predictor of patient response to therapy ${ }^{233,353}$.

In recent years, several new immune-based therapies have received FDA approval or are currently in clinical trials for the treatment of melanoma 16,17,195,222,347,354. Immunotherapeutics that target the programmed death receptor (PD-1) axis have shown promise in clinical trials and have been FDA approved for the treatment of melanoma 219,222,228-230,355,356. In healthy tissue, the PD-1 axis prevents autoimmunity by downregulating the activity of cytotoxic T cells ${ }^{202,225}$. Melanoma cells co-opt this mechanism by overexpressing the receptor ligand, PD-L1, which when bound to the PD-1 receptor on cytotoxic T cells deactivates the T cells and blunts the antitumor immune response ${ }^{202,225}$. Several different monoclonal antibodies (mAb) against PD-1 have been developed and have shown significant improvements in survival compared to chemotherapy, but response rates vary between 20 and 40\% 194,195. Poor response is often attributed to depleted T cell populations within the tumor, as treatment efficacy is dependent on a robust $T$ cell population ${ }^{219,222,228}$. Various attempts have been made to increase the response rate, including combining PD-1 with other checkpoint inhibitors like CTLA-4 17,30,233,357, adjuvant therapies such as radiation or CpG injections ${ }^{256,349}$, and adoptive cell transfer of activated T cells ${ }^{226}$. However, these combinations can be associated with severe side effects related to autoimmunity ${ }^{16}$.

Here, we evaluate a novel adjuvant for immunotherapy: high pressure pulsed ultrasound with intravenously administered microbubbles (US). High intensity focused 
ultrasound (HIFU) ablation has been associated with antitumor immune responses in several types of cancer, including pancreatic ${ }^{358}$, prostate ${ }^{243,244,263}$, colon ${ }^{258}$, kidney 252,264 and breast 253,359 . However, recent studies have shown that mechanical ablation 258 or partial thermal ablation ${ }^{254}$ produce a more powerful antitumor immune response, indicating that techniques which enhance tumor antigen exposure without causing heat denaturation of the tumor tissue may be more efficacious. Low intensity ultrasound and microbubble therapy has been shown to enhance the delivery of immune cells to tumors ${ }^{266}$, induce hypoxic cell death due to decreased perfusion ${ }^{268}$, and enhance tumor resident CD4 and CD8 T cell populations ${ }^{267}$. However, the mechanisms are poorly understood. Here, we identify the relative contributions of the adaptive and innate immune systems, as well as T cell trafficking and proliferation, in ultrasound-mediated antitumor immune response.

In this chapter, we show that low intensity pulsed ultrasound and microbubble therapy significantly enhances tumor growth control and animal survival in the B16F10 mouse model of melanoma, but does not synergize with concurrent PD-1 therapy. Furthermore, this growth control is abolished in immunocompromised Rag-1 knockout mice and under pharmaceutical blockade of $\mathrm{T}$ cell trafficking, indicating that $\mathrm{T}$ cell recruitment to the tumor is a primary mechanism in ultrasound-mediated tumor growth control. Ultrasound and microbubble therapy does not produce significant increases in $T$ cell proliferation at one week post-treatment, and may induce tumor resident immune cell death, which may provide an explanation for the poor synergy with concurrent PD-1 therapy. This work identifies non-thermal, low intensity pulsed ultrasound and microbubble therapy as an immune adjuvant that out-performs FDA-approved PD-1 therapy and provides a foundation for future studies examining the mechanisms and synergistic potential of this new therapy.

\section{EXPERIMENTAL METHODS}

\section{STUDY DESIGN}

\section{ULTRASOUND AND ANTI PD-1 THERAPY}

To test the hypothesis that ultrasound and microbubble therapy can increase $T$ cell tumor infiltration and enhance the efficacy of anti PD-1 therapy, 8-10 week old C57Bl6 (Jackson) mice were subcutaneously inoculated with 50,000 B16F10 melanoma cells and divided into four groups: untreated control, ultrasound and microbubble therapy (US), aPD-1 therapy ( $\mathrm{QPD}-1$ ) and ultrasound and microbubble therapy combined with $\alpha \mathrm{PD}-1$ therapy (US+aPD-1). $\alpha \mathrm{PD}-1$ (RPM1-14, Abcam) was given intraperitonealy on days 11,14 and 17 at a dosage of $250 \mu \mathrm{g}$ in $0.1 \mathrm{~mL}$ sterile water. This dosage has been used heavily in the literature and produces significant alterations in T cell responses without significant side effects $226,233,237$. Animals were treated with ultrasound on day 12 as described in the following sections. Tumors were measured on alternating days until day 12 and daily thereafter. On day 18, animals were euthanized 
and tumors were removed (Fig 3.1). Flow cytometry was performed to analyze the tumor-resident immune cell populations. All animal experiments were carried out at the University of Virginia following National Institutes of Health guidelines and local Institutional Animal Care and Use Committee regulations.

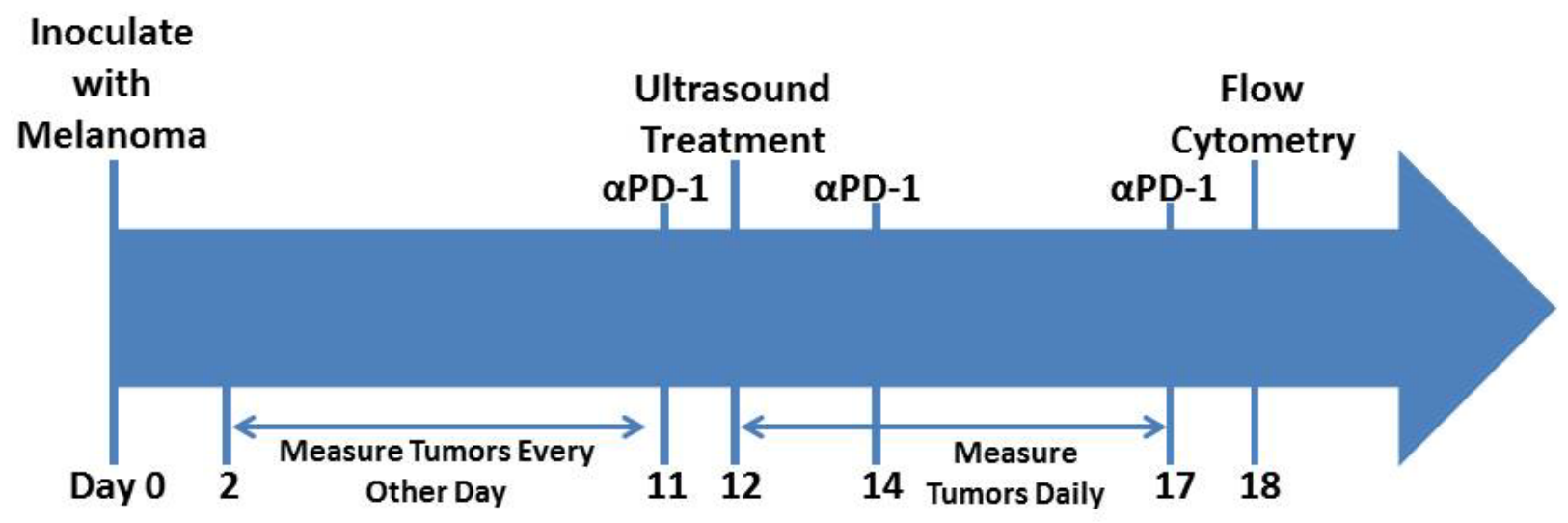

Figure 3.1: Experimental design: ultrasound and $\alpha$ PD-1 therapy

\section{ULTRASOUND IN AN IMMUNOCOMPROMISED MOUSE MODEL}

To test the hypothesis that ultrasound and microbubble therapy inhibits tumor outgrowth via an interaction with the innate immune system, 8-10 week old Rag1 knockout (B6.129S7-Rag1tm1Mom/J, Jackson) mice were used. This wellcharacterized mouse model does not produce produce mature B or T lymphocytes but has non-leaky vasculature ${ }^{360,361}$. Mice were subcutaneously inoculated with 50,000 B16F10 melanoma cells and divided into two groups: untreated control and ultrasound and microbubble therapy (US). Animals were treated with ultrasound on day 12 as described in the following sections. Tumors were measured on alternating days until day 12 and daily thereafter. On day 18, animals were euthanized and tumors were removed (Fig 3.2). Flow cytometry was performed to analyze the tumor-resident immune cell populations. All animal experiments were carried out at the University of Virginia following National Institutes of Health guidelines and local Institutional Animal Care and Use Committee regulations. 


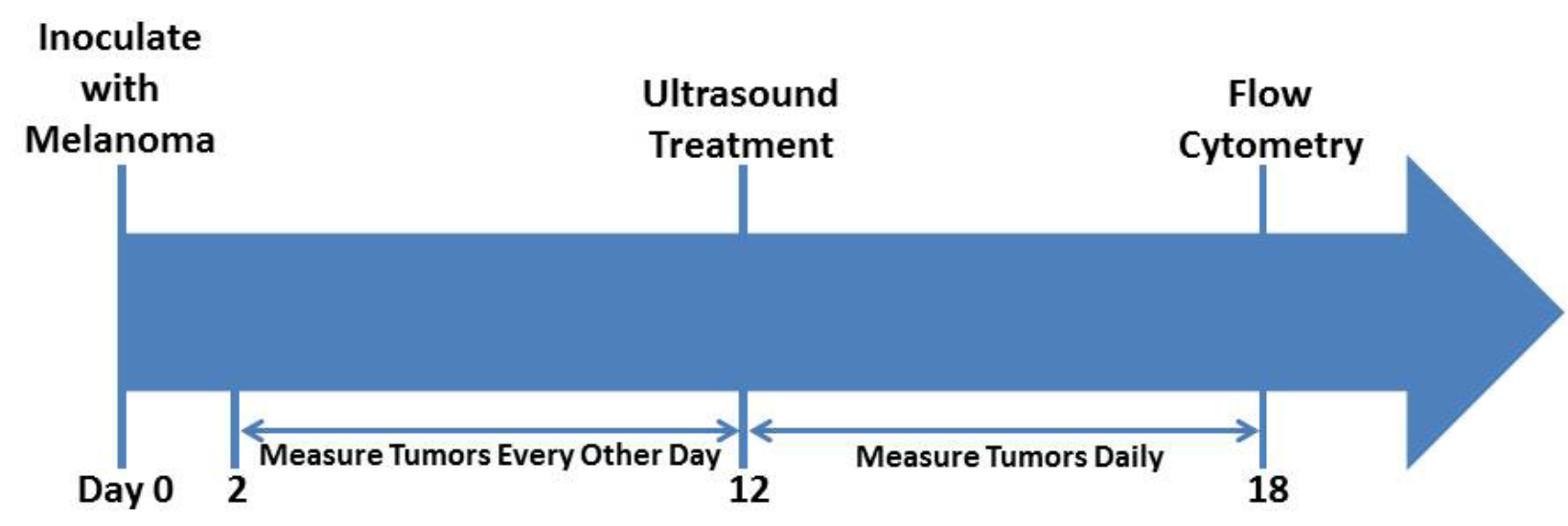

Figure 3.2: Experimental design: ultrasound in an immunocompromised mouse model

\section{ULTRASOUND AND T-CELL TRAFFICKING}

To test the hypothesis that ultrasound and microbubble therapy increases $T$ cell trafficking to the treated tumor, 8-10 week old C57BI6 (Jackson) mice were subcutaneously inoculated with 50,000 B16F10 melanoma cells and divided into three groups: adoptive cell transfer and FTY720 (ACT+FTY), ultrasound and microbubble therapy and adoptive cell transfer (US+ACT) and ultrasound and microbubble therapy, adoptive cell transfer, and FTY720 (US+ACT+FTY). Freshly isolated naïve T cells were collected from PMEL-1 mice (B6.Cg-Thy1a/Cy Tg(TcraTcrb)8Rest/J, Jackson) as previously described ${ }^{362}$ and injected intravenously $\left(10^{6}\right.$ cells in $0.1 \mathrm{~mL}$ sterile DPBS) on day 10 . These cells express a rearranged $T$ cell receptor transgene specific for the mouse homologue (pmel-17) of human SILV (gp100), which is involved in pigment synthesis and is expressed in B16 melanoma cells ${ }^{362}$. Adoptively transferred PMEL-1 cells can also be distinguished from host $T$ cells by the presence of the T lymphocyte specific Thy1a (Thy1.1) allele ${ }^{362}$. FTY720 (Sigma) was added to animals' drinking water at concentration of $2 \mu \mathrm{g} / \mathrm{mL}$ on day 11 and replaced every 3 days thereafter. This treatment protocol has been well characterized and is known to prevent $T$ cell egress from the lymph nodes without significant side effects ${ }^{363-365}$. Animals were treated with ultrasound on day 12 as described in the following sections. Tumors were measured on alternating days until day 12 and daily thereafter. On day 18, animals were euthanized and tumors, spleens and draining and non-draining inguinal lymph nodes were removed (Fig 3.3). Flow cytometry was performed to analyze the tumor-, spleen- and lymphresident immune cell populations. Ki67 was used as a marker of cellular proliferation. All animal experiments were carried out at the University of Virginia following National Institutes of Health guidelines and local Institutional Animal Care and Use Committee regulations. 


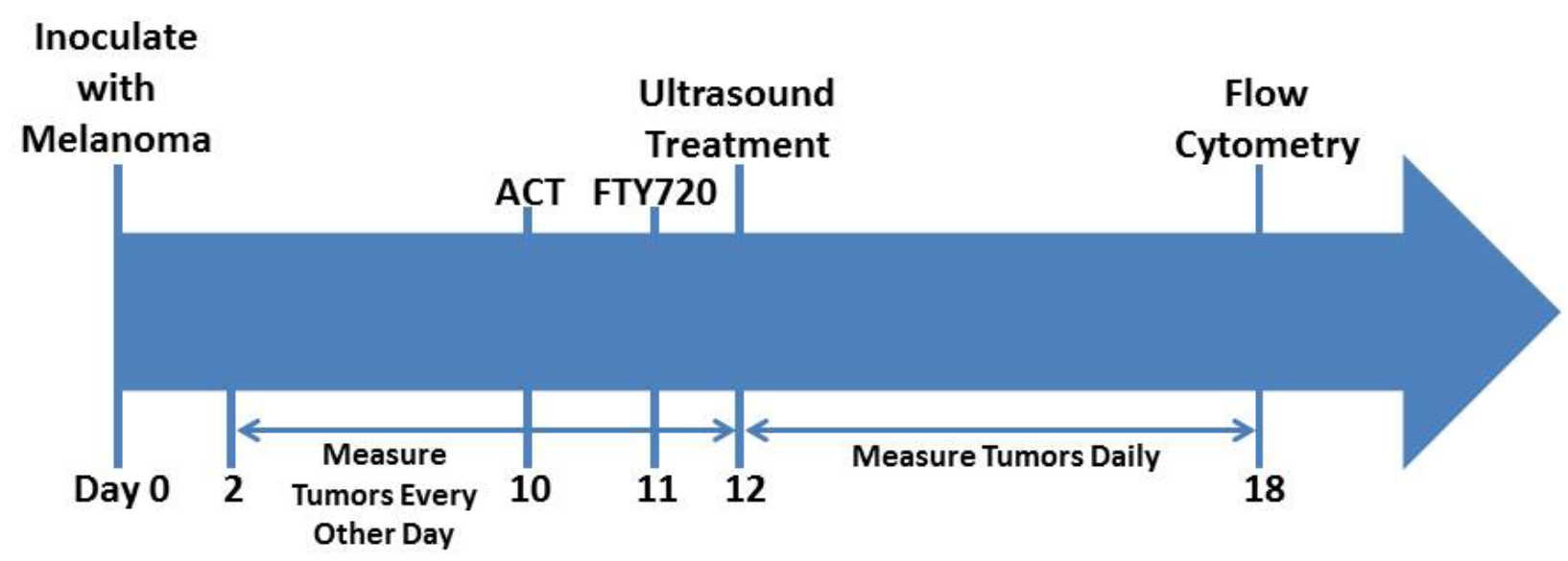

Figure 3.3: Experimental design: ultrasound and t-cell trafficking

\section{MICROBUBBLE FABRICATION AND CHARACTERIZATION}

As described in Aim 1.

\section{CELL CULTURE}

B16F10 mouse melanoma cells were obtained from the American Type Culture Collection (ATCC). Cells were cultured in Roswell Park Memorial Institute1640 medium (RPMI, Gibco) with 1.5\% 1M Hepes buffer (Gibco) and 5\% fetal bovine serum (FBS, Gibco) added. For each experiment, cells were brought back from cryopreservation to eliminate variations in in vitro passage number. Cells were maintained at $37^{\circ} \mathrm{C}$ and $5 \%$ $\mathrm{CO} 2$.

\section{TUMOR INOCULATION}

B16F10 cells were removed from the culture flask with 0.25\% Trypsin-EDTA (Gibco), centrifuged at $1000 \mathrm{rpm}$ for 5 minutes at $4^{\circ} \mathrm{C}$ and resuspended in sterile Dulbecco's phosphate-buffered saline at a concentration of 500000 cells $/ \mathrm{mL}$. Cells were kept on ice for no longer than 1 hour.

Animals' right flanks were shaved and depilated four days prior to tumor inoculation under $2 \%$ isoflurane (Piramel). On the day of inoculation, animals were anesthesized with an intraperitoneal injection of Ketamine (40 mg/kg, Fort Dodge) and Dexdomitor $(0.2 \mathrm{mg} / \mathrm{kg}$, Pfizer) in sterilized water. A $30 \mathrm{~g}$ needle was used to inject 0.1 $\mathrm{mL}$ (50000 cells) of the cell suspension over 30 seconds. The needle was left in place for an additional 30 seconds following completion of the injection, and the animals remained under anesthesia for 30 minutes to avoid disturbing the injection site. The anesthesia was reversed using Antisedan (2 mg/kg, Zoetis). 


\section{ULTRASOUND TREATMENT}

On the day of treatment, animals were anesthetized with an intraperitoneal injection of Ketamine (40 mg/kg, Fort Dodge) and Dexdomitor (0.2 mg/kg, Pfizer) in sterilized water. The tail vein of each animal was cannulated for intravenous administration of an MB solution. A water-based ultrasound gel (Aquasonic 100, Parker Laboratories, Inc.) was applied to the skin above the flank tumor, and a 0.75-in-diameter 1-MHz unfocused transducer (A314S, Panametrics) was coupled to the skin. The MBs $\left(10^{5} \mathrm{MBs} / \mathrm{g}\right.$ body weight in $0.3 \mathrm{ml}$ of $0.9 \%$ saline) were continuously infused intravenously with a pump (PHD 2000, Harvard Apparatus) for the duration of the experiment. The pulsing protocol shown in Fig3.4 was generated on waveform generator (AFG-310, Tektronix, Inc.) and amplified by a 55-dB radiofrequency power amplifier (ENI 3100LA, Electronic Navigation Industries) at a 1-V peak-to-peak (1.2 MPa) amplitude. We have previously shown that this protocol does not produce measurable heating within the tumor ${ }^{85}$.

\section{1,000 Sinusoids}

In $1 \mathrm{~ms}$

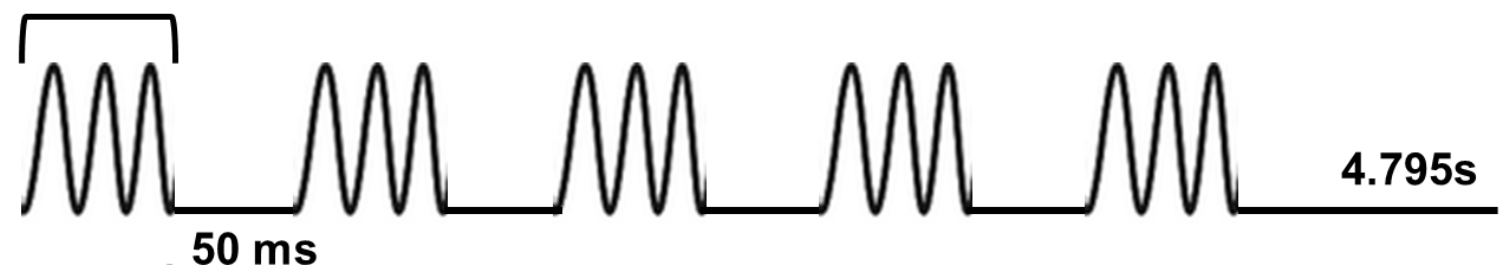

Figure 3.4: Schematic of ultrasound pulsing protocol. Bursts were repeated every 5 seconds for 60 minutes with continuous MB infusion. $\mathrm{DC}=0.0001$

\section{TUMOR MONITORING}

Subcutaneous tumors were measured using calipers every other day until day 12 and every day thereafter. Tumor volume was calculated according to the formula $\mathrm{V}=$ $\frac{1}{2}$ (length $*$ width $^{2}$ ). When tumors reached $2040 \mathrm{~mm}^{3}$, animals were euthanized. Percent tumor growth inhibition was calculated as \% TGI $=100 \times \frac{\mathrm{V}_{\mathrm{c}-} \mathrm{V}_{\mathrm{t}}}{\mathrm{V}_{\mathrm{c}}-\mathrm{V}_{\mathrm{o}}}$ where $\mathrm{V}_{\mathrm{c}}$ and $\mathrm{V}_{\mathrm{t}}$ are the median tumor volumes in the treated groups and control group, respectively, on day 15 and $\mathrm{V}_{\mathrm{o}}$ is the median tumor volume at Day 12.

\section{FLOW CYTOMETRY}

Mice were euthanized with pentobarbital $(0.5 \mu \mathrm{l} / \mathrm{g}$ body weight) on day 18 . Tumors, spleens, draining and non-draining lymph nodes (both brachial and inguinal where possible) were removed within 20 minutes and were placed in sterile DPBS on ice. Tumors were homogenized and tumor infiltrating lymphocytes (TIL) were isolated with Lympholyte-M (Cedarlane). Spleens were homogenized and treated with Red 
Blood Cell Lysing Buffer Hybri-Max (Sigma). Lymph nodes were homogenized. Flow samples were acquired on the FACSCanto II (BD Biosciences) for aPD-1 experiments and on a Cytoflex S (Beckman Coulter) for all other experiments. Flow data was analyzed with FlowJo software (Tree Star). Cell counts were determined using 123count eBeads (Affymetrix). Flow cytometry gating strategies and antibodies used are shown in Tables 3.1 and 3.2, respectively (See FigS1 and S2 for additional information). Thy1.1 was used to identify PMEL-1 T cells. It is important to note that all measured T cell subsets were activated, as determined by CD44 staining.

Table 3.1: Flow cytometry gating strategy.

\begin{tabular}{|c|c|c|c|c|c|c|c|}
\hline CD4 & Treg & CD8 & DC & Myeloid & NK & M1 & non-M1 \\
\hline Live & Live & Live & Live & Live & Live & Live & Live \\
\hline Singlet & Singlet & Singlet & Singlet & Singlet & Singlet & Singlet & Singlet \\
\hline CD45+ & CD45+ & CD45+ & CD45+ & CD45+ & CD45+ & CD45+ & CD45+ \\
\hline CD44+ & CD44+ & CD44+ & NK1.1- & NK1.1- & NK1.1+ & NK1.1- & NK1.1- \\
\hline CD4+ & CD4+ & CD8+ & CD11c hi & CD11c- & & CD11b+ & CD11b+ \\
\hline & CD25+ & & & CD11b+ & & F4/80+ & F4/80+ \\
\hline & Foxp3+ & & & & & MHC II+ & MHC II- \\
\hline & & & & & & CD86+ & CD86- \\
\hline & & & & & & CD80+ & CD80- \\
\hline
\end{tabular}

Table 3.2: Flow cytometry antibodies.

\begin{tabular}{|c|c|c|c|}
\hline Ab & Clone & Company & Channel(s) \\
\hline \multicolumn{2}{|c|}{ Live/Dead } & Life Technologies & Aqua \\
\hline CD45.2 & 104 & eBioscience & FITC \\
\hline CD4 & RM4-5 & eBioscience & e450 \\
\hline CD8 & $53-6.7$ & BioLegend & BV650 \\
\hline CD44 & IM7 & eBioscience & AF700 \\
\hline CD25 & PC61.5 & eBioscience & PE \\
\hline Thy1.1 & HIS51 & eBioscience & APC, PE-Cy7 \\
\hline Foxp3 & FJK-16s & eBioscience & PerCP-Cy5.5 \\
\hline Ki67 & SolA15 & eBioscience & PE-Cy7 \\
\hline CD11b & M1/70 & eBioscience & APCe780 \\
\hline CD11c & N418 & eBioscience & PE \\
\hline F4/80 & BM8 & BioLegend & BV421 \\
\hline MHC II & M5/114.15.2 & eBioscience & FITC \\
\hline CD80 & $16-10 A 1$ & BD Biosciences & PerCP-Cy5.5 \\
\hline CD86 & PO3.1 & eBioscience & PE \\
\hline CD206 & C068C2 & BioLegend & AF647 \\
\hline CD44 & IM7 & eBioscience & PE \\
\hline CD8 & $53-6.7$ & eBioscience & PE-Cy7 \\
\hline CD25 & PC61.5 & eBioscience & APC \\
\hline
\end{tabular}




\section{ULTRASOUND AND ANTI-PD-1 THERAPY}
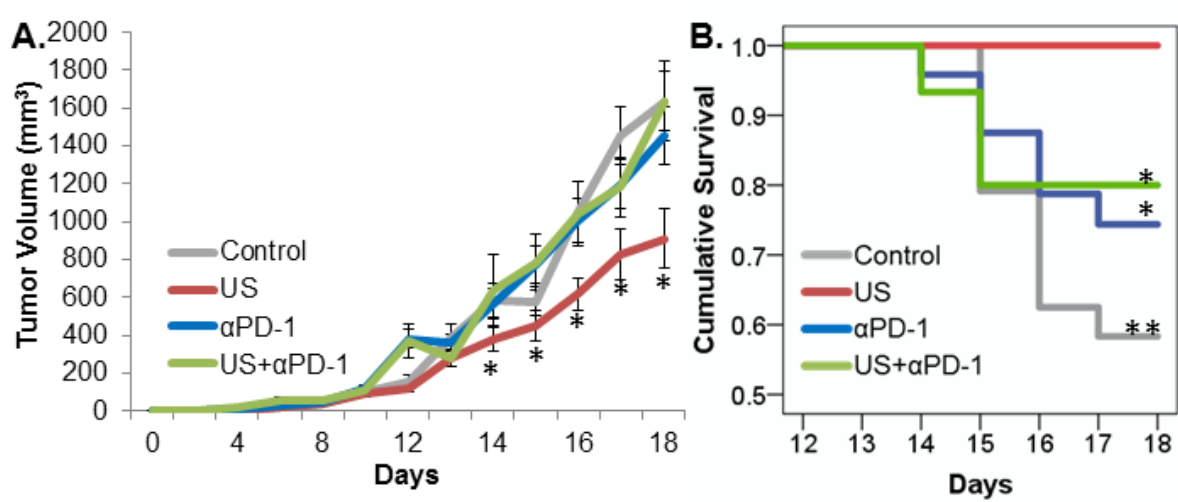

Figure 3.5: Ultrasound alone improves tumor growth control and survival in a mouse model of melanoma. Mice treated with pulsed ultrasound and microbubbles (US) on day 12 showed significantly depressed tumor outgrowth by day 14 (A) compared to untreated controls and mice treated with aPD-1 alone. By day 15, animals treated with US alone had significantly smaller tumors than all other groups. All animals treated with ultrasound alone survived to day 18 (B), a significant improvement compared to all other groups. * indicates $\mathrm{p} \leq 0.05$, * indicates $\mathrm{p} \leq 0.001, \mathrm{n} \geq 16$.
To test the hypothesis that ultrasound can act as an adjuvant for anti-PD-1 therapy, we compared tumor growth and survival in animals treated with US or PD-1 therapy alone or in combination. There were no significant interactions or main effects in tumor growth control. US significantly improved tumor growth control compared to all other groups by day 14 , two days after US

treatment (Fig3.5A). By day 15, when the first control animal was euthanized, US treatment had produced a $76 \%$ tumor growth inhibition compared to untreated controls. Animals receiving either PD-1 alone or in combination with US showed no improvement in tumor growth control versus untreated controls (Fig3.5A). The US treatment group

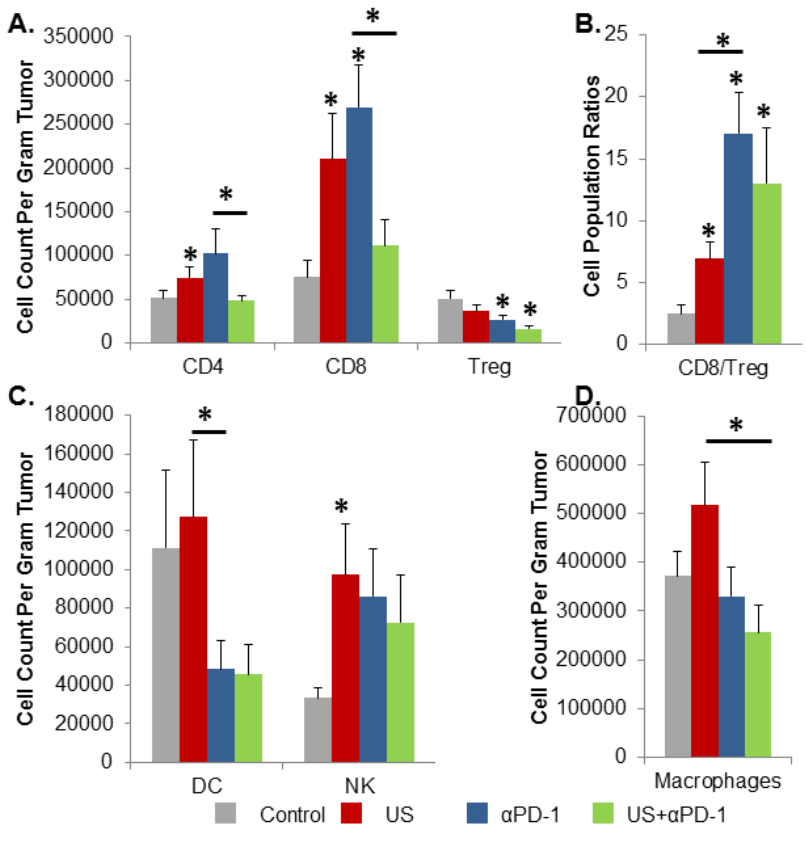

had $100 \%$ survival (Fig3.5B), compared to

\section{Figure 3.6: Ultrasound or anti-PD-1 alone} improve the tumor immune profile, but this benefit is abolished when they are combined. US or aPD-1 alone increased the numbers of activated CD4 and CD8 cells within the tumor, and aPD-1 decreased the number of Tregs as well. US+aPD-1 recruited fewer CD4 and CD8 cells than aPD-1 therapy alone, but decreased the Treg population compared to controls. (A) aPD-1 treatment produced the largest CD8/Treg ratio, significantly higher than either control or US alone. (B) Animals receiving aPD-1 had fewer DCs than those receiving US, US significantly increased the number of NK cells compared to control. (C)Tumors treated with US+aPD-1 had less macrophages than those treated with US alone. * indicates $p<0.05$ versus control, unless otherwise noted. $n \geq 8$. 
$58 \%$ for untreated controls ( $\mathrm{p}=0.001), 75 \%$ for PD- $1(\mathrm{p}=0.016)$ and $82 \%$ for US+PD-1 $(p=0.036)$. There was no significant difference between survival in control, PD-1 and US+PD-1 treatment groups.

To identify the mechanism of US-induced tumor growth control, we first analyzed the tumor-resident immune cell population 7 days post ultrasound treatment. There was a significant interaction effect between PD-1 and ultrasound therapies (Fig3.6A) on both CD4 ( $p=0.035)$ and CD8 T cells $(p=0.002)$. Animals treated with US had a larger tumor resident population of CD4 cells than those that received no treatment $(p=0.05)$, while animals given PD-1 alone had the greatest CD4 cell population, a 2.1-fold increase (FI) compared to the combination therapy $(p=0.04)$. A similar trend was observed in the CD8 compartment. Either therapy alone produced a larger CD8 population than untreated controls (2.8 FI, $p=0.000$ for US and $3.6 \mathrm{FI}, \mathrm{p}=0.003$ for PD- 1 ), and PD- 1 therapy induced a 2.4-fold increase in CD8s compared to the US+PD-1 $(p=0.02)$. There was no interaction effect on tumor resident Treg populations (Fig3.6A), but the main effect of PD-1 therapy was significant $(p=0.005)$. US treatment did not affect Treg populations, but the PD-1 and US+PD-1 groups showed a $0.53(p=0.016)$ and $0.31(p=0.02)$ fold decrease (FD), respectively, compared to untreated controls. The main effect of PD-1 therapy on the $C D 8 / T c e l l$ ratio was significant $(p=0.000$, Fig3.6B), with the PD- 1 and US+PD-1 groups averaging a CD8/Tcell ratio of $17(p=0.000)$ and $13(p=0.001)$, respectively, compared to 2.5 in controls. The CD8/Tcell ratio in the PD-1 group was significantly higher $(p=0.05)$ than in the US group, which generated a CD8/Tcell ratio of 6.9 ( $p=0.03$ vs control). US also enhanced the innate immune response to the tumor (Fig3.6C,D). The main effect of PD-1 was significant $(p=0.018$, Fig3.6C) in DC populations within the tumor, and the US group had a 2.6 FI in DC numbers compared to the PD-1 cohort $(p=0.001)$. Additionally, US increased the tumor resident NK population by 2.9 -fold compared to untreated controls ( $p=0.005$, Fig3.6C). PD-1 also produced a significant main effect $(p=0.04$, Fig3.6D) on the macrophage population within the tumor. US treated tumors showed a $2 \mathrm{FI}(p=0.019)$ compared to those treated with US+PD-1.

\section{ULTRASOUND IN AN IMMUNOCOMPROMISED MODEL}

To evaluate the hypothesis that US-induced tumor growth control is dependent on the adaptive immune system, we analyzed tumor growth and animal survival in an immunocompromised Rag-1 knockout mouse, which lack mature B and T cells. Tumors treated with US showed no growth control (Fig3.7A) and US treatment did not confer a survival benefit (Fig3.8A) when compared to untreated controls. 

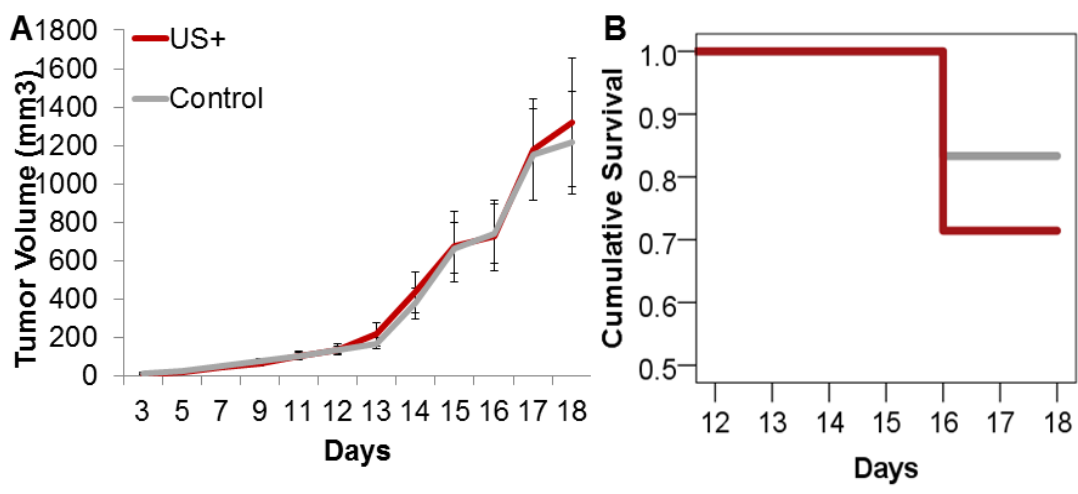

Figure 3.7: Ultrasoundinduced tumor growth control is lost in an immunocompromised mouse model. In a Rag-1 KO mouse, US treatment does not improve tumor growth control $(A)$ or animal survival $(B)$. There is no significant difference between US and control. $\mathrm{n}=15$.

To test the hypothesis that US treatment can enhance innate immune response to a tumor in the absence of the adaptive immune system, we next examined the immune cell profiles within US treated tumors 7 days post-treatment and compared them to untreated controls. US treatment did not affect DC, NK or non-M1 cell populations but significantly decreased the total number of M1 macrophages (Fig3.8A, $\mathrm{p}=0.002$ ) and decreased the M1/Non-M1 ratio from 0.70 to 0.52 (Fig3.8B, $\mathrm{p}=0.03$ ).
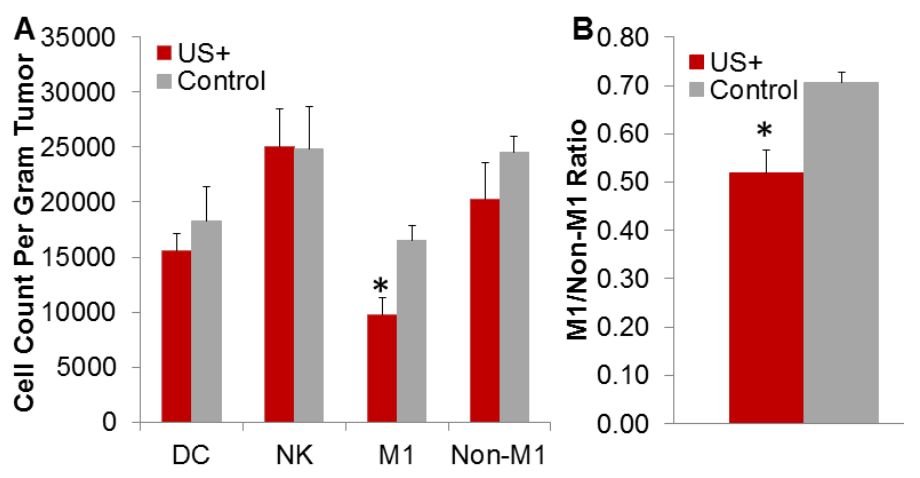

Figure 3.8: Ultrasound has minimal effects on the tumor immune profile in an immunocompromised mouse model. In a Rag-1 KO mouse, US does not affect the populations of DC, NK or non-M1cells within the tumor, but significantly decreases $\mathrm{M} 1$ macrophages (A) and alters the M1/Non-M1 ratio (B). * indicates $p<0.05 . n=15$.

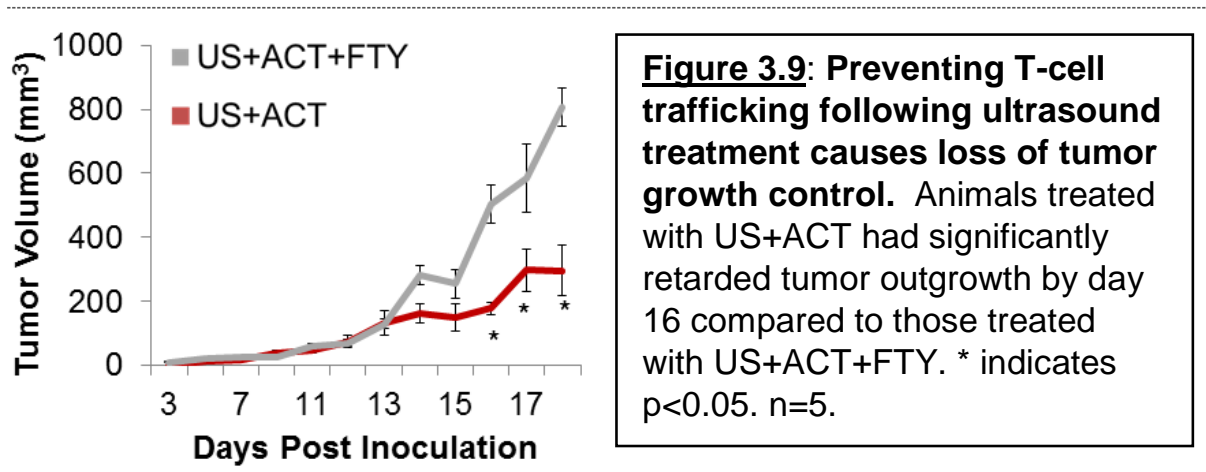

US+ACT+FTY versus US+ACT (Fig3.9). When trafficking was pharmacologically blocked using FTY720, tumor growth was significantly higher by day 16 , four days after US treatment. Animals treated with US+ACT had $70 \%$ TGI by day $18(\mathrm{p}=0.0009)$. There 


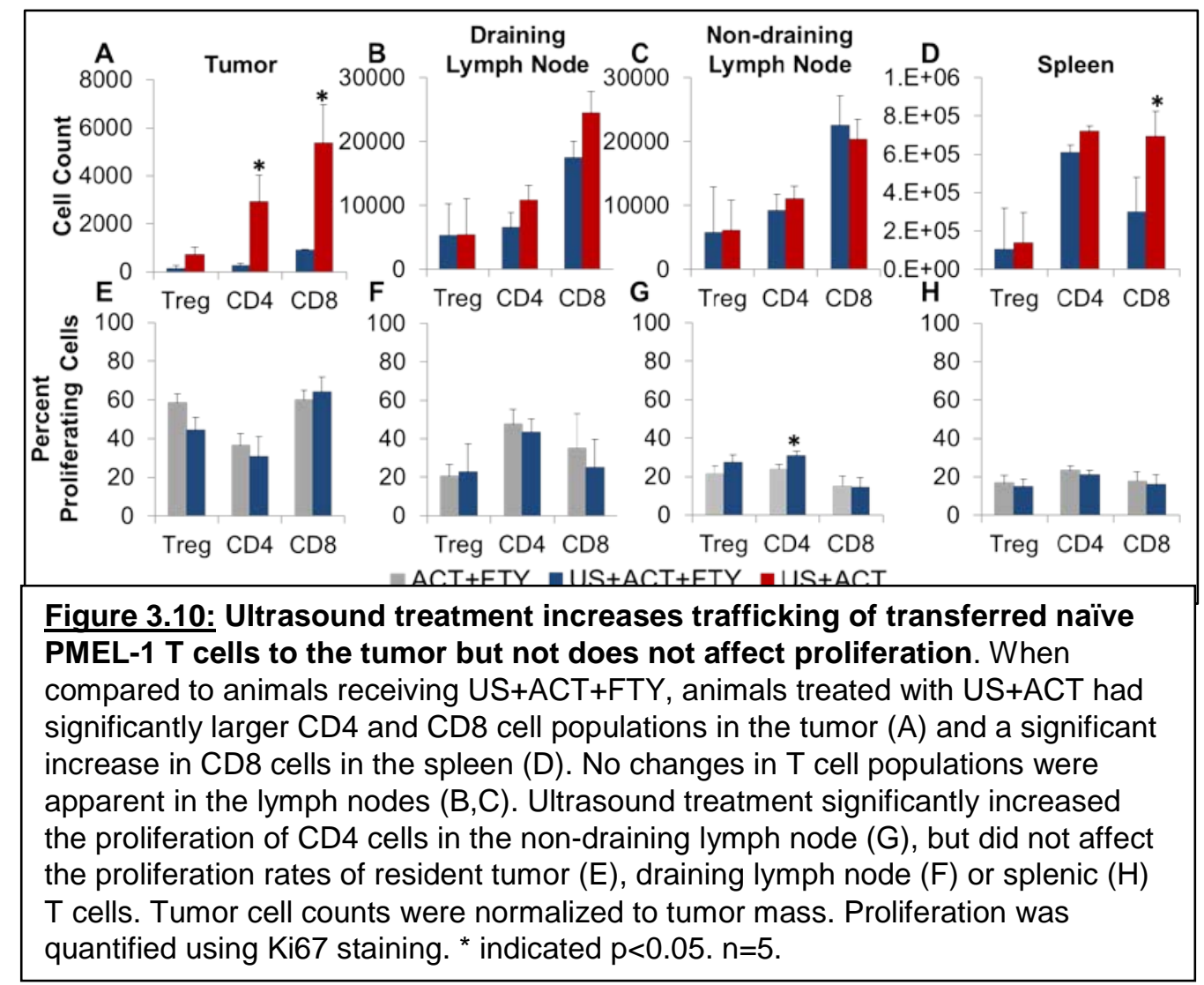

was no difference in animal survival between groups; both groups had $100 \%$ survival (data not shown).

To evaluate the hypothesis that US induces T cell trafficking, we examined the tumor, lymphatic and splenic $T$ cell populations to determine the fate of naïve PMEL-1 T cells given via adoptive transfer in US treated animals with (US+ACT+FTY) or without (US+ACT) pharmaceutical blockade of trafficking with FTY720 (Fig3.10A-D). In the presence of $T$ cell trafficking, US treatment produced an increase in the number of CD4 (11.5 FI, p=0.02) and CD8 (6.0 FI, p=0.04) cells within the tumor, compared to the US+ACT+FTY group (Fig3.10A). There were no differences in the T cell populations within either the draining (Fig3.10B) or non-draining (Fig3.10C) lymph nodes. However, splenic CD8 cells were elevated in the US+ACT group (2.3 FI, p=0.05, Fig3.10D).

To test the hypothesis that US can induce T cell proliferation with the tumor, lymphatic and splenic T cell populations, we compared Ki67 expression in PMEL-1 T cells (Fig3.10E-H) delivered via adoptive transfer in animals treated with FTY720 (ACT+FTY) alone versus US in combination with FTY720 (US+ACT+FTY). T cell proliferation was highest in the tumor and lowest in the spleen (46 and 19\% across all cell types and groups, respectively). A modest but significant increase in proliferation in CD4 cells was noted in non-draining lymph nodes (Fig3.10G), but US treatment had no other effects on $\mathrm{T}$ cell proliferation at this timepoint. 
Prior to initializing treatment (Day 10-12 depending on experimental setup), animals' tumor volumes were calculated and outliers were identified and removed from the study. Animals were randomly sorted into treatment groups such that the average tumor size between groups prior to treatment was not statistically different. Data in Figure 3.5A and 3.6 were analyzed using a two-way ANOVA in IBM SPSS Statistics (Windows Version 22.0). Outliers were assessed by inspection of a boxplot, and were eliminated from further analysis. In data sets that showed a statistically significant interaction effect, pairwise comparisons and an analysis of simple main effects was conducted. In data sets that did not show a statistically significant interaction effect, pairwise comparisons and an analysis of main effects was performed. Data in Figure 3.5B and 3.7B were analyzed using Kaplan-Meier survival analysis, followed by a Mantel-Cox log rank test. Mantel-Cox log rank pairwise comparisons were also performed for data in Figure 3.5B. All survival analyses were performed with IBM SPSS Statistics (Windows Version 22.0). Data in Figure 3.7A, 3.8, 3.9 and 3.10 were analyzed using an independent-samples t-test in Microsoft Excel (2010). Unless otherwise noted, significance was set at $p<0.05$.

\section{DISCUSSION AND CONCLUSIONS}

Here we show that low intensity pulsed ultrasound in conjunction with microbubbles significantly enhances tumor growth control and animal survival in the B16F10 mouse model of melanoma, outperforming PD-1 therapy. While both PD-1 and US therapy increased the tumor resident CD4 and CD8 cell populations, this did not translate to a functional benefit in PD-1 treated animals. Furthermore, the combination of US and PD-1 demonstrated significant improvement in the CD8/Treg ratio compared to untreated controls but otherwise showed a profound negative synergy by all other metrics when compared to either treatment alone. US treatment also increased tumor resident DC, NK and macrophage populations. In immunocompromised Rag-1 knockout mice, US-induced tumor growth control was abolished, but US induced a significant decrease in the number of tumor resident M1-macrophages. When T cell trafficking was pharmaceutically blocked using FTY720, US-induced tumor growth control was similarly abolished and tumor resident CD4 and CD8 populations dropped. Lymph node populations showed no change, but splenic CD8 cell populations decreased. US treatment had no measurable effect on T cell proliferation 7 days post-treatment. From this collection of data, we conclude that low intensity pulsed ultrasound and microbubble therapy acts as a T-cell mediated immune adjuvant, but that more work is necessary to understand how it may best be applied and combined with other therapies.

While PD-1 therapy produces dramatic tumor regression in a subset of patients and has a better safety profile than CTLA-4, response rates are still low 219,232 , and it has not demonstrated great success as a monotherapy in preclinical trials ${ }^{233,349,357}$. In agreement with our PD-1 monotherapy results, Dai et al showed that 4 intratumoral 
doses of $250 \mu \mathrm{g}$ of $\alpha \mathrm{PD}-1$ (compared to our protocol of 3 i.p. injections of $250 \mu \mathrm{g}$ ) did not produce survival benefits or tumor growth control in the same B16F10 melanoma model 237. When given at these doses, PD-1 therapy must be combined with an additional therapy to generate tumor growth control and improvements in survival. Dai et al showed that a complete response was obtained in $20 \%$ of mice but only when animals received 6 doses of a synergistic combination of three mAbs (PD-1, CTLA-4,CD137) ${ }^{237}$. Similarly, Curran et al demonstrated no survival benefit with 3 i.p. doses of $250 \mu \mathrm{g}$ PD-1 in the less immunogenic B16BI6 melanoma model, but achieved a complete response in $25 \%$ of animals when PD-1 was combined with vaccination with irradiated B16 melanoma cells expressing Flt3-ligand ${ }^{233}$. Again in agreement with our work, Curran et al showed that PD-1 therapy increases tumor resident CD4 and CD8 T cell populations while decreasing Treg populations, leading to a large increase in the CD8/Tcell ratio ${ }^{233}$. Other groups have demonstrated that PD-1 monotherapy is not a curative therapy in various tumor models and must be combined with other therapies to be effective ${ }^{357,366}$.

Since large tumor infiltrating CD4 and CD8 T cell populations are associated with favorable prognosis ${ }^{222,347}$ and PD-1 therapy is most effective in tumors with a robust CD8 T cell population ${ }^{227,367}$, adjuvant therapies which can increase baseline CD4 and CD8 T cell tumor infiltration may synergize well with PD-1. Thermal HIFU ablation increases CD4 ${ }^{257,260,264,358}$ and CD8 ${ }^{252,260-262}$ cell infiltration within treated tumors. Mechanical ablation ${ }^{246,258,263}$ and sparse-scan ablation protocols ${ }^{254}$ that do not fully denature tumor antigens improve antigen presenting cell (APC) activation compared to thermal protocols, indicating that non-thermal approaches may be more effective in generating an antigen-specific systemic antitumor response. Furthermore, low intensity pulsed ultrasound with microbubbles (US) has been shown to enhance delivery of NK cells to tumors ${ }^{266}$, increase $T$ cell infiltration in response to ultrasound-induced tumor ischemia ${ }^{268}$, and increase the CD8/Treg ratio while providing modest tumor growth control ${ }^{267}$. Our results with US, demonstrating improved tumor growth control, increased CD4, CD8, and NK populations, and increased CD8/Treg ratios, agree nicely with this body of work and further support the hypothesis that US should synergize with PD-1 therapy.

Our prior work in a subcutaneous glioma model demonstrated that the US protocol used here did not produce a temperature rise in treated tumors, but did generate a significant reduction (but not elimination) in blood flow ${ }^{85}$. The protocol significantly increased apoptotic and necrotic cells within the tumor, but this did not translate to growth control ${ }^{85}$. We chose this protocol because it maintained blood flow (total cessation would reduce immune access to the tumor) and induced non-thermal tumor cell death. Similar work by another group in a melanoma model demonstrated significant increases in tumor infiltrating lymphocytes (CD45+CD3+) following temporary tumor ischemia and upregulation of HIF1 $\alpha^{268}$. HIF1 $\alpha$ is known to enhance neutrophilendothelial binding, CD8 T cell activity ${ }^{368}$, M1 macrophage differentiation ${ }^{369}$ and DC activation ${ }^{369}$. Given that US-induced tumor growth control was abolished in the Rag-1 knockout model, a CD8 and DC mediated HIF1 $\alpha$-induced antitumor immune response could provide a unifying theory. However, HIF1a has been shown to increase the 
expression of PD-L1 (but not PD-1) on myeloid-derived suppressor cells (MDSC) and B16F10 cells ${ }^{370,371}$ as well as other cancer cell lines ${ }^{371}$, abrogating CD8 T cell cytotoxicity ${ }^{370,371}$. When tumor cells were incubated with either aPD-1 or QPD-L1, CD8 T cell cytotoxicity was restored ${ }^{370,371}$. If US-induced tumor growth control was mediated via HIF1a, we would expect a positive synergy with PD-1, which was not the case here. Therefore, it is unlikely that hypoxia-induced upregulation of HIF1 $\alpha$ is the underlying mechanism in US-mediated tumor growth inhibition.

We propose three potential mechanisms by which US may enhance the antitumor immune response and thus synergize with PD-1 therapy: endothelial cell activation, microvascular disruption and tumor stroma disruption (Table 1 in the Background). In noting that the only significant difference between US and PD-1 monotherapies in tumor resident immune cell populations is a decrease in DCs in PD-1 treated animals, we may propose a hypothesis: US-induced tumor growth inhibition is a result of increased antigen expression via either microvascular or tumor stroma disruption which, in the presence of a sustained intratumoral DC population capable of recognizing antigens, leads to increased overall DC-mediated antigen presentation and downstream CD8 T cell activation. The observed loss of US-induced tumor growth control in both immunocompromised and pharmaceutical T cell trafficking blockade supports this hypothesis, as does the loss of CD4 and CD8 T cell populations within US-treated tumors following trafficking blockade. This hypothesis also agrees with a study by Bandyopadhyay et al, which demonstrated that low intensity ultrasound (mild thermal rise, without microbubbles) caused release of tumor antigen and reversed tumor-induced T cell tolerance ${ }^{372}$. Given this hypothesis, we would expect accumulation of CD4 and CD8 cells within the draining lymph nodes during pharmaceutical trafficking blockade ${ }^{363}$. However, at the time point used here (day 7 ), the lymphocyte population in lymph nodes in FTY720 treated animals returns to normal levels (following a temporary surge) due to reduced release of naïve T cells from the thymus ${ }^{363}$. T cell proliferation within the lymph nodes occurs within a few days following antigen exposure ${ }^{363}$, so an earlier time point (day 2-3) may better identify changes in proliferation within the lymph nodes. Additionally, the T cell staining panel used here does not distinguish between effector and memory cells, and may be masking important changes in the composition of the T cell populations within the lymph nodes ${ }^{373}$.

The observed negative synergy between PD-1 and US treatments could be due in part to the significant reduction in tumor infiltrating DCs observed in the PD-1 treated animals. However, it is more likely that blockade of the PD-1 pathway upregulated a secondary immunosuppressive pathway that subsequently reduced the efficacy of the US treatment. It has been shown that PD-1 treatment can increase the expression of alternate immunosuppressive pathways ${ }^{231}$, including TIM-3, and for this reason, multiple checkpoint blockade is more effective than monotherapy 221,223,224,238. However, this positive synergy is dependent on timing and non-redundancy in the targeted pathways ${ }^{222,374 .}$ 
Ultimately, more experimentation is needed to further elucidate the mechanisms underlying US-mediated tumor growth control. This work emphasizes the need for preclinical models for testing potential immune-based synergies and has identified and begun to address a previously unknown knowledge gap. One important conclusion from this study is that metrics used to predict outcomes in checkpoint-based therapies (such as CD8/Treg ratio) are not appropriate for US-mediated responses, since the PD-1 monotherapy group had a significantly higher CD8/Treg ratio compared to US monotherapy but did not perform as well in growth inhibition or survival. Moving forward, greater temporal resolution with respect to immune cell proliferation and trafficking would be highly informative. This data, taken 7 days post US treatment, nicely captures T cell behavior in the tumor (excellent agreement between CD8 T cell levels and tumor growth control) but does not accurately measure the behavior of innate immune cells, whose activity peaks between 2 and 24 hours post-insult, depending on the cell type. Furthermore, several studies have determined that the timing of adjuvant therapies can determine synergy ${ }^{374}$, underscoring the importance of a more detailed understanding of immune cell behavior in the hours and days following US treatment. For now, we have demonstrated that non-thermal low intensity pulsed ultrasound and microbubble therapy acts as an immune adjuvant in the B16F10 mouse melanoma model, improving tumor growth control and animal survival.

This is the first study to characterize the relative contributions of the adaptive and innate immune systems, as well as T cell trafficking and proliferation, in ultrasoundmediated immune responses. We are also the first to attempt to combine ultrasound with an immunotherapeutic. In determining that increased T-cell trafficking following ultrasound and microbubble treatment produces significant tumor growth control, we have identified the previously unknown mechanism by which low-intensity ultrasound acts as an immune adjuvant. Future studies may build upon this work to identify potential synergies and improve the efficacy of other immune-based therapies.

\section{FUTURE DIRECTIONS}

This work has identified a novel application for low intensity ultrasound and microbubble therapy but has also identified a knowledge gap in the field. While we demonstrated antitumor immune responses, tumor growth inhibition and survival in animals treated with ultrasound alone, the predicted synergy between US and PD-1 therapies did not materialize and in fact demonstrated negative synergy in terms of immune cell infiltration. We have determined that US-mediated tumor growth inhibition is dependent on T cell trafficking, but more questions remain.

While the proliferation data from day 7 post-treatment did not reveal any USinduced effects, earlier timepoints (days 1-3) may provide a better picture, as innate and DC proliferation responses occur much earlier. Additionally, CellTrace technology could be utilized to gain a better understanding of the fate of adoptively transferred T cells. Longer time points, particularly in the B16-OVA model, may provide valuable information about memory cell formation. 
An alternate way to assess memory cell formation or antigen specificity of cytotoxic T cell activity is the use of rechallenge ${ }^{238}$ or bilateral tumor inoculations ${ }^{237}$. The abscopal effect, describing an aggressive systemic antitumor response following treatment of the primary tumor, is the holy grail of immunotherapy and is mediated by tumor-specific cytotoxic $T$ cells and can be assessed in animals bearing multiple tumors when only one tumor is treated ${ }^{242}$. Tumor-specific memory cell formation can be evaluated in animals that have received a curative immunotherapy, followed by reinoculation with the same tumor line ${ }^{263}$. Alternately, cell lines containing a known tumor antigen (such as B16-OVA) can be used to assess memory cell antigen specificity.

In addition to evaluating immune cell antigen specificity and memory function, further characterization of the impact of various immune cell types could provide valuable information. While it is apparent that US induces greater levels of $T$ cell trafficking to the tumor, these incoming $T$ cells may be more active than tumor-resident $\mathrm{T}$ cells, providing an additional benefit. INFy production assays would provide a metric of T cell activity, as increased INFY production is associated with increased cytotoxic activity ${ }^{226}$. In vivo depletion of individual cell types, such as DCs, may also identify key mediators of US-induced tumor growth control and help identify the mechanism of the observed negative synergy ${ }^{375}$.

To identify molecular changes that may be occurring following US treatment, there are several other experiments that could be conducted. Several studies have measured significant changes in chemokine profiles in the blood following low intensity pulsed ultrasound ${ }^{238,376}$. Additionally, endothelial cell activation within the vasculature can significantly increase immune cell capture and infiltration within the tumor. Analysis of endothelial cellular adhesion molecules that modulate this process, such as VCAM and $\mathrm{E}$-Cadherin ${ }^{377}$, may also provide valuable insight regarding the mechanism of USmediated antitumor immunity. Although we did not observe a global temperature rise in the tumor during treatment ${ }^{85}$, it is well documented that HSP70 is released from tumor cells following thermal ablation ${ }^{244,247,248}$ and it may be possible that it is upregulated in response to local temperature increases occurring during microbubble cavitation. Along the same lines, analysis of HIF1 $\alpha$ expression following US treatment would definitively determine whether hypoxia is playing a role. PD-L1 expression in the tumor before and after US expression may also provide information about the observed negative synergy between US and PD-1 therapy, and may also inform future decisions regarding the PD1 dosing regimen.

The US treatment protocol could also be further modified to enhance its efficacy. While the timeline of the initial treatment is dictated by the development of functional vasculature within the tumor due to the use of microbubbles, additional US treatments would likely provide further benefit ${ }^{12}$. Judicious combination with another therapy may also improve the response. Low intensity ultrasound and microbubble therapy has been used to increase the delivery of drugs ${ }^{157}$, genes ${ }^{164}$ and cells ${ }^{266}$ to tumors, and could be combined with traditional chemotherapeutics, genetic material or engineered immune cells. Other work has demonstrated that the dosing regimen of combinatorial 
immunotherapies can determine their synergistic effect ${ }^{222}$, so comparing concurrent versus successive combinations may be of benefit as well.

Immunotherapeutics are becoming the new gold standard in the treatment of many types of cancer, but poor response rates hinder their efficacy, generating a need for adjuvant therapies that can improve the baseline immune cell response to the tumor. As an adjuvant, low intensity pulsed ultrasound and microbubble therapy has a low barrier to translation given that HIFU is already approved for the treatment of several types of cancer ${ }^{378}$. Furthermore, this therapy is non-invasive and extremely low risk. We hope that this work will provide the foundation for clinical translation of low intensity ultrasound for immunomodulation. 

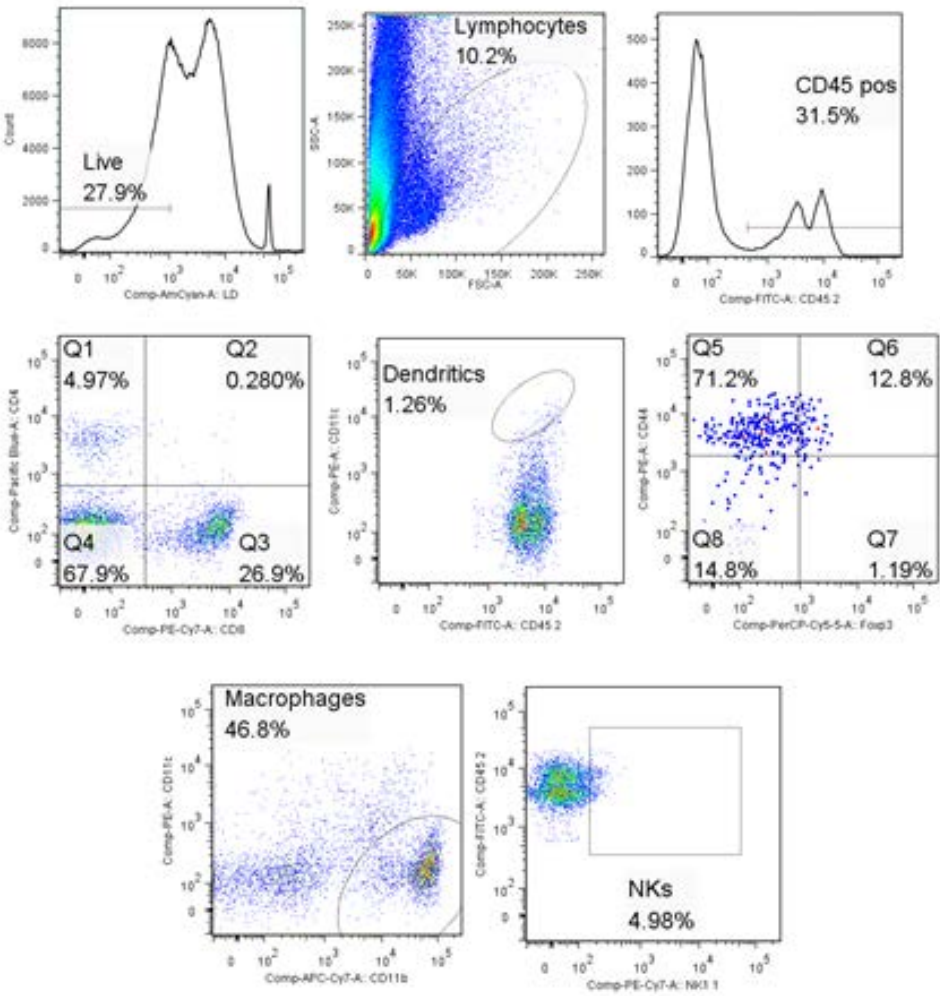

Figure S1: Gating strategy for T cells, natural killer cells, dendritic cells and macrophages 

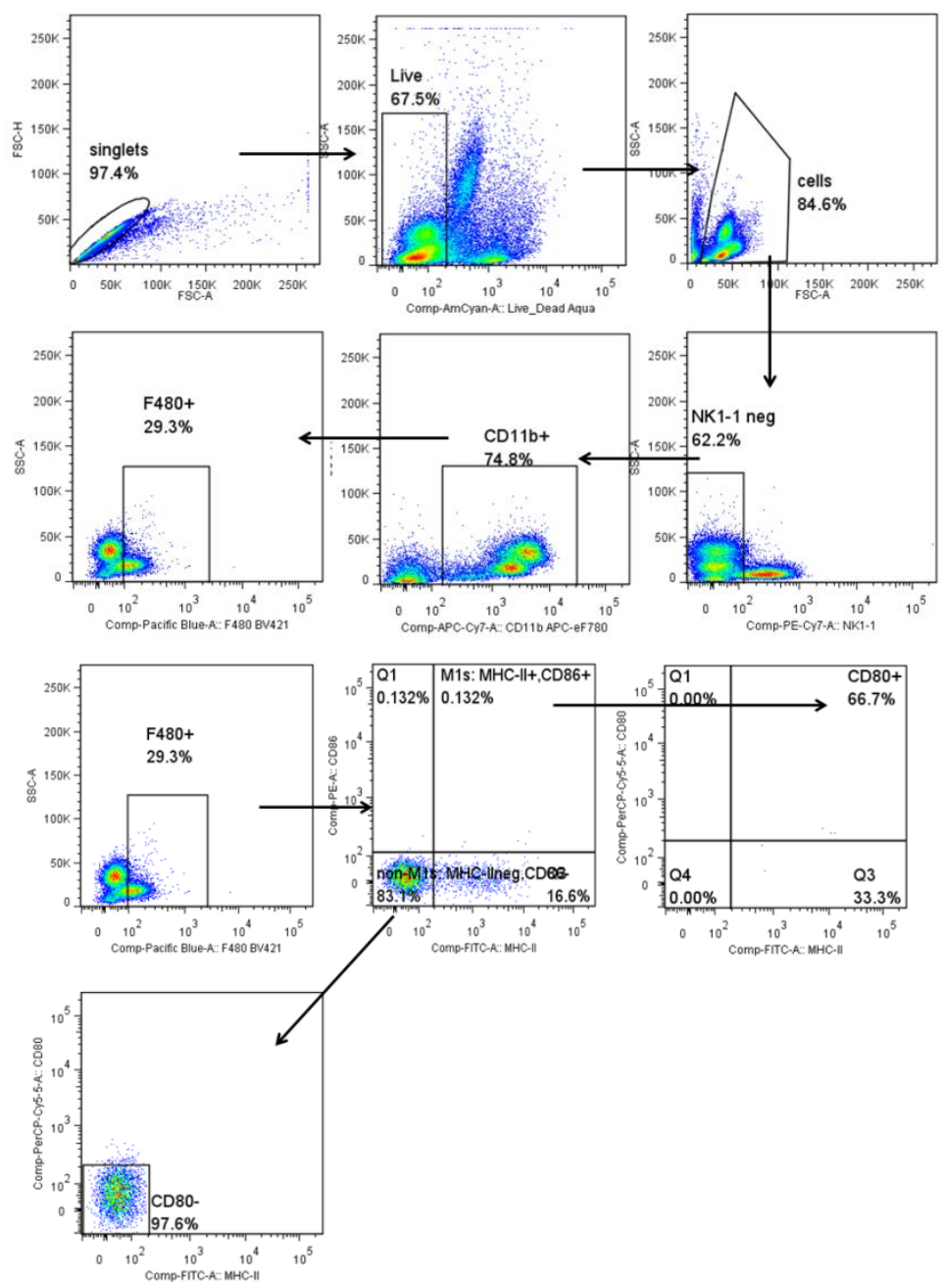

Figure S2: Gating strategy for M1/non-M1 macrophages 
1. Raymond SB, Skoch J, Hynynen K, Bacskai BJ. Multiphoton imaging of ultrasound/Optison mediated cerebrovascular effects in vivo. J Cereb Blood Flow Metab. 2007;27(2):393-403. doi:10.1038/sj.jcbfm.9600336.

2. Cho EE, Drazic J, Ganguly M, Stefanovic B, Hynynen K. Two-photon fluorescence microscopy study of cerebrovascular dynamics in ultrasound-induced blood-brain barrier opening. J Cereb Blood Flow Metab. 2011;31(9):1852-1862. doi:10.1038/jcbfm.2011.59.

3. Treat LH, McDannold N, Zhang Y, Vykhodtseva N, Hynynen K. Improved anti-tumor effect of liposomal doxorubicin after targeted blood-brain barrier disruption by MRI-guided focused ultrasound in rat glioma. Ultrasound Med Biol. 2012;38(10):1716-1725. doi:10.1016/j.ultrasmedbio.2012.04.015.

4. Jagannathan J, Sanghvi NT, Crum LA, et al. High-intensity focused ultrasound surgery of the brain: part 1--A historical perspective with modern applications. Neurosurgery. 2009;64(2):201-10-1. doi:10.1227/01.NEU.0000336766.18197.8E.

5. Kovacs Z, Werner B, Rassi A, Sass JO, Martin-Fiori E, Bernasconi M. Prolonged survival upon ultrasound-enhanced doxorubicin delivery in two syngenic glioblastoma mouse models. J Control Release. May 2014. doi:10.1016/j.jconrel.2014.05.033.

6. Yoshida M, Kawakami S, Kono Y, et al. Enhancement of the anti-tumor effect of DNA vaccination using an ultrasound-responsive mannose-modified gene carrier in combination with doxorubicin-encapsulated PEGylated liposomes. Int J Pharm. 2014;475(1-2):401-407. doi:10.1016/j.ijpharm.2014.09.005.

7. Bauer H-C, Krizbai IA, Bauer H, Traweger A. "You Shall Not Pass"-tight junctions of the blood brain barrier. Front Neurosci. 2014;8:392. doi:10.3389/fnins.2014.00392.

8. Alizadeh AA, Aranda V, Bardelli A, et al. Toward understanding and exploiting tumor heterogeneity. Nat Med. 2015;21(8):846-853. doi:10.1038/nm.3915.

9. Wong HL, Wu XY, Bendayan R. Nanotechnological advances for the delivery of CNS therapeutics. Adv Drug Deliv Rev. November 2011. doi:10.1016/j.addr.2011.10.007.

10. Prados MD, Byron SA, Tran NL, et al. Toward precision medicine in glioblastoma: the promise and the challenges. Neuro Oncol. May 2015. doi:10.1093/neuonc/nov031.

11. Burgess A, Shah K, Hough O, Hynynen K. Focused ultrasound-mediated drug delivery through the blood-brain barrier. Expert Rev Neurother. 2015;15(5):477-491. doi:10.1586/14737175.2015.1028369.

12. Aryal M, Vykhodtseva N, Zhang Y-Z, Park J, McDannold N. Multiple treatments with liposomal doxorubicin and ultrasound-induced disruption of blood-tumor and blood-brain barriers improve outcomes in a rat glioma model. J Control Release. 2013;169(1-2):103111. doi:10.1016/j.jconrel.2013.04.007.

13. Wilczewska AZ, Niemirowicz K, Markiewicz KH, Car H. Nanoparticles as drug delivery systems. Pharmacol Reports. 2012;64(5):1020-1037. doi:10.1016/S1734-1140(12)709015 . 
14. Danhier F, Ansorena E, Silva JM, Coco R, Le Breton A, Préat V. PLGA-based nanoparticles: An overview of biomedical applications. J Control Release. February 2012. doi:10.1016/j.jconrel.2012.01.043.

15. Rueth NM, Cromwell KD, Cormier JN. Long-term Follow-up for Melanoma Patients: Is There Any Evidence of a Benefit? Surg Oncol Clin N Am. 2015;24(2):359-377. doi:10.1016/j.soc.2014.12.012.

16. Shah DJ, Dronca RS. Latest Advances in Chemotherapeutic, Targeted, and Immune Approaches in the Treatment of Metastatic Melanoma. Mayo Clin Proc. 2014;89(4):504519. doi:10.1016/j.mayocp.2014.02.002.

17. Simeone E, Ascierto PA. Immunomodulating antibodies in the treatment of metastatic melanoma: the experience with anti-CTLA-4, anti-CD137, and anti-PD1. J Immunotoxicol. 9(3):241-247. doi:10.3109/1547691X.2012.678021.

18. Rosberger DF, Coleman DJ, Silverman R, Woods S, Rondeau M, Cunningham-Rundles S. Immunomodulation in choroidal melanoma: reversal of inverted CD4/CD8 ratios following treatment with ultrasonic hyperthermia. Biotechnol Ther. 1994;5(1-2):59-68.

19. Puhalla S, Elmquist W, Freyer D, et al. Unsanctifying the sanctuary: challenges and opportunities with brain metastases. Neuro Oncol. 2015;17(5):639-651.

doi:10.1093/neuonc/nov023.

20. Neurological Disorders: Public Health Challenges.; 2006.

21. Substance Abuse and Mental Health Services Administration. Results from the 2012 National Survey on Drug Use and Health : Summary of National Findings.; 2013.

22. Stewart WF, Ricci JA, Chee E, Hahn SR, Morganstein D. Cost of lost productive work time among US workers with depression. JAMA. 2003;289(23):3135-3144. doi:10.1001/jama.289.23.3135.

23. Pardridge WM. The blood-brain barrier: bottleneck in brain drug development. NeuroRx. 2005;2(1):3-14. doi:10.1602/neurorx.2.1.3.

24. Pardridge WM, Boado RJ. Reengineering Biopharmaceuticals for Targeted Delivery across the Blood-Brain Barrier. Vol 503. 1st ed. Elsevier Inc.; 2012. doi:10.1016/B978-012-396962-0.00011-2.

25. Deleu D, Northway MG, Hanssens Y. Clinical pharmacokinetic and pharmacodynamic properties of drugs used in the treatment of Parkinson's disease. Clin Pharmacokinet. 2002;41(4):261-309. doi:10.2165/00003088-200241040-00003.

26. Lonser RR, Sarntinoranont M, Morrison PF, Oldfield EH. Convection-enhanced delivery to the central nervous system. J Neurosurg. 2015;122(March):697-706. doi:10.3171/2014.10.JNS14229.Disclosure.

27. Morrison PF. Distributed Models of drug Kinetics. In: Principles of Clinical Pharmacology. ; 2007:107-128. doi:10.1016/B978-012369417-1/50049-3.

28. Bobo RH, Laske DW, Akbasak A, Morrisont PF, Dedrickt RL, Oldfield EH. Convectionenhanced delivery of macromolecules in the brain. 1994;91(March):2076-2080. 
29. Marks WJ, Bartus RT, Siffert J, et al. Gene delivery of AAV2-neurturin for Parkinson's disease: a double-blind, randomised, controlled trial. Lancet Neurol. 2010;9(12):11641172. doi:10.1016/S1474-4422(10)70254-4.

30. Marks WJ, Ostrem JL, Verhagen L, et al. Safety and tolerability of intraputaminal delivery of CERE-120 (adeno-associated virus serotype 2-neurturin) to patients with idiopathic Parkinson's disease: an open-label, phase I trial. Lancet Neurol. 2008;7(5):400-408. doi:10.1016/S1474-4422(08)70065-6.

31. Ramaswamy S, McBride JL, Han I, et al. Intrastriatal CERE-120 (AAV-Neurturin) protects striatal and cortical neurons and delays motor deficits in a transgenic mouse model of Huntington's disease. Neurobiol Dis. 2009;34(1):40-50. doi:10.1016/j.nbd.2008.12.005.

32. Gasmi M, Brandon EP, Herzog CD, et al. AAV2-mediated delivery of human neurturin to the rat nigrostriatal system: long-term efficacy and tolerability of CERE-120 for Parkinson's disease. Neurobiol Dis. 2007;27(1):67-76. doi:10.1016/j.nbd.2007.04.003.

33. Ren X, Zhang T, Gong X, Hu G, Ding W, Wang X. AAV2-mediated striatum delivery of human CDNF prevents the deterioration of midbrain dopamine neurons in a 6hydroxydopamine induced parkinsonian rat model. Exp Neurol. 2013;248:148-156. doi:10.1016/j.expneurol.2013.06.002.

34. Gonzales-Portillo GS, Sanberg PR, Franzblau M, et al. Mannitol-enhanced delivery of stem cells and their growth factors across the blood-brain barrier. Cell Transplant. 2014;23(4-5):531-539. doi:10.3727/096368914X678337.

35. Lee HJ, Zhang Y, Pardridge WM. Blood-brain barrier disruption following the internal carotid arterial perfusion of alkyl glycerols. J Drug Target. 2002;10(6):463-467. doi:10.1080/1061186021000038337.

36. Bhattacharjee AK, Kondoh T, Nagashima T, Ikeda M, Ehara K, Tamaki N. Quantitative analysis of papaverine-mediated blood-brain barrier disruption in rats. Biochem Biophys Res Commun. 2001;289(2):548-552. doi:10.1006/bbrc.2001.6029.

37. Black KL, Cloughesy T, Huang SC, et al. Intracarotid Infusion of RMP-7, a Bradykinin Analog, and Transport of Gallium-68 Ethylenediamine Tetraacetic Acid into Human Gliomas. Vol 86.; 1997. doi:10.3171/jns.1997.86.4.0603.

38. Lochhead JJ, Thorne RG. Intranasal delivery of biologics to the central nervous system. Adv Drug Deliv Rev. 2012;64(7):614-628. doi:10.1016/j.addr.2011.11.002.

39. Costantino HR, Illum L, Brandt G, Johnson PH, Quay SC. Intranasal delivery: Physicochemical and therapeutic aspects. Int J Pharm. 2007;337(1-2):1-24. doi:10.1016/j.jpharm.2007.03.025.

40. Ikeda M, Bhattacharjee AK, Kondoh T, Nagashima T, Tamaki N. Synergistic effect of cold mannitol and $\mathrm{Na}(+) / \mathrm{Ca}(2+)$ exchange blocker on blood-brain barrier opening. Biochem Biophys Res Commun. 2002;291(3):669-674. doi:10.1006/bbrc.2002.6495.

41. Zylber-Katz E, Gomori JM, Schwartz A, Lossos A, Bokstein F, Siegal T. Pharmacokinetics of methotrexate in cerebrospinal fluid and serum after osmotic bloodbrain barrier disruption in patients with brain lymphoma. Clin Pharmacol Ther. 2000;67(6):631-641. doi:10.1067/mcp.2000.106932. 
42. Lipsman N, Schwartz ML, Huang $\mathrm{Y}$, et al. MR-guided focused ultrasound thalamotomy for essential tremor: a proof-of-concept study. Lancet Neurol. 2013;12(5):462-468.

doi:10.1016/S1474-4422(13)70048-6.

43. Elias WJ, Huss D, Voss T, et al. A pilot study of focused ultrasound thalamotomy for essential tremor. N Engl J Med. 2013;369(7):640-648. doi:10.1056/NEJMoa1300962.

44. Dallapiazza R, McKisic MS, Shah B, Elias WJ. Neuromodulation for movement disorders. Neurosurg Clin N Am. 2014;25(1):47-58. doi:10.1016/j.nec.2013.08.002.

45. Dobrakowski PP, Machowska-Majchrzak AK, Labuz-Roszak B, Majchrzak KG, Kluczewska E, Pierzchała KB. MR-guided focused ultrasound: a new generation treatment of Parkinson's disease, essential tremor and neuropathic pain. Interv Neuroradiol. 20(3):275-282.

46. Hynynen K, Mcdannold N. Imaging - guided Focal Opening of the Blood-Brain Barrier in Rabbits 1. 2001;(4).

47. Hynynen K, McDannold N, Vykhodtseva N, Jolesz FA. Non-invasive opening of BBB by focused ultrasound. Acta Neurochir Suppl. 2003;86:555-558.

48. McDannold N, Vykhodtseva N, Raymond S, Jolesz F a., Hynynen K. MRI-guided targeted blood-brain barrier disruption with focused ultrasound: Histological findings in rabbits. Ultrasound Med Biol. 2005;31(11):1527-1537. doi:10.1016/j.ultrasmedbio.2005.07.010.

49. Runge VM, Clanton JA, Price AC, et al. The use of Gd DTPA as a perfusion agent and marker of blood-brain barrier disruption. Magn Reson Imaging. 1985;3(1):43-55. doi:10.1016/0730-725X(85)90008-6.

50. Liu H-LL, Wai Y-YY, Chen W-SS, et al. Hemorrhage detection during focused-ultrasound induced blood-brain-barrier opening by using susceptibility-weighted magnetic resonance imaging. Ultrasound Med Biol. 2008;34(4):598-606.

doi:10.1016/j.ultrasmedbio.2008.01.011.

51. Cheng AL, Batool S, McCreary CR, et al. Susceptibility-weighted imaging is more reliable than T2*-weighted gradient-recalled echo mri for detecting microbleeds. Stroke. 2013;44(10):2782-2786. doi:10.1161/STROKEAHA.113.002267.

52. Fry W, Fry F, Meyers R, Eggleton RC. The Use of Ultrasound In Neurosurgery. In: The Third International Conference on Medical Electronics. ; 1960.

53. Hynynen $\mathrm{K}$, Jolesz $\mathrm{F}$ a. Demonstration of potential noninvasive ultrasound brain therapy through an intact skull. Ultrasound Med Biol. 1998;24(2):275-283. doi:10.1016/S03015629(97)00269-X.

54. Rooney $\mathrm{J}$ a. Shear as a mechanism for sonically induced biological effects. $\mathrm{J}$ Acoust Soc Am. 1972;52(6):1718-1724.

55. Davidson BJ, Riley N. MICROSTREAMING. East. 1971;15:217-233.

56. Chen H, Kreider W, Brayman AA, Bailey MR, Matula TJ. Blood vessel deformations on microsecond time scales by ultrasonic cavitation. Phys Rev Lett. 2011;106(3):34301.

57. Wu J, Ross JP, Chiu J-F. Reparable sonoporation generated by microstreaming. J 
Acoust Soc Am. 2002;111(3):1460. doi:10.1121/1.1420389.

58. Juffermans LJM, van Dijk A, Jongenelen CAM, et al. Ultrasound and microbubbleinduced intra- and intercellular bioeffects in primary endothelial cells. Ultrasound Med Biol. 2009;35(11):1917-1927. doi:10.1016/j.ultrasmedbio.2009.06.1091.

59. Sheikov N, McDannold N, Sharma S, Hynynen K. Effect of focused ultrasound applied with an ultrasound contrast agent on the tight junctional integrity of the brain microvascular endothelium. Ultrasound Med Biol. 2008;34(7):1093-1104. doi:10.1016/j.ultrasmedbio.2007.12.015.

60. Deng J, Huang Q, Wang F, et al. The role of caveolin-1 in blood-brain barrier disruption induced by focused ultrasound combined with microbubbles. $J \mathrm{Mol}$ Neurosci. 2012;46(3):677-687. doi:10.1007/s12031-011-9629-9.

61. Xia C, Zhang Z, Xue Y, Wang P, Liu Y. Mechanisms of the increase in the permeability of the blood-tumor barrier obtained by combining low-frequency ultrasound irradiation with small-dose bradykinin. J Neurooncol. 2009;94(1):41-50. doi:10.1007/s11060-009-9812-9.

62. Meijering BDM, Juffermans LJM, van Wamel A, et al. Ultrasound and microbubbletargeted delivery of macromolecules is regulated by induction of endocytosis and pore formation. Circ Res. 2009;104(5):679-687. doi:10.1161/CIRCRESAHA.108.183806.

63. Nhan T, Burgess A, Cho EE, Stefanovic B, Lilge L, Hynynen K. Drug delivery to the brain by focused ultrasound induced blood-brain barrier disruption: Quantitative evaluation of enhanced permeability of cerebral vasculature using two-photon microscopy. J Control Release. 2013;172(1):274-280. doi:10.1016/j.jconrel.2013.08.029.

64. Chen $\mathrm{H}$, Konofagou $\mathrm{EE}$. The size of blood-brain barrier opening induced by focused ultrasound is dictated by the acoustic pressure. J Cereb Blood Flow Metab. 2014;34(7):1197-1204. doi:10.1038/jcbfm.2014.71.

65. McDannold N, Vykhodtseva N, Hynynen K. Targeted disruption of the blood-brain barrier with focused ultrasound: association with cavitation activity. Phys Med Biol. 2006;51(4):793-807. doi:10.1088/0031-9155/51/4/003.

66. Samiotaki G, Vlachos F, Tung YS, Feshitan J, Borden M, Konofagou EE. Pressure and microbubble size dependence study of focused ultrasound-induced blood-brain barrier opening reversibility in vivo. AIP Conf Proc. 2012;1481:300-306. doi:10.1063/1.4757352.

67. Park J, Zhang Y, Vykhodtseva N, Jolesz F a, McDannold NJ. The kinetics of blood brain barrier permeability and targeted doxorubicin delivery into brain induced by focused ultrasound. J Control Release. 2012;162(1):134-142. doi:10.1016/j.jconrel.2012.06.012.

68. Howles GP, Bing KF, Qi Y, Rosenzweig SJ, Nightingale KR, Johnson GA. Contrastenhanced in vivo magnetic resonance microscopy of the mouse brain enabled by noninvasive opening of the blood-brain barrier with ultrasound. Magn Reson Med. 2010;64(4):995-1004. doi:10.1002/mrm.22411.

69. McDannold N, Arvanitis CD, Vykhodtseva N, Livingstone MS. Temporary disruption of the blood-brain barrier by use of ultrasound and microbubbles: safety and efficacy evaluation in rhesus macaques. Cancer Res. 2012;72(14):3652-3663. doi:10.1158/0008-5472.CAN12-0128. 
70. Downs ME, Buch A, Sierra C, et al. Long-Term Safety of Repeated Blood-Brain Barrier Opening via Focused Ultrasound with Microbubbles in Non-Human Primates Performing a Cognitive Task. PLoS One. 2015;10(5):e0125911. doi:10.1371/journal.pone.0125911.

71. Hynynen K, McDannold N, Sheikov N a, Jolesz F a, Vykhodtseva N. Local and reversible blood-brain barrier disruption by noninvasive focused ultrasound at frequencies suitable for trans-skull sonications. Neuroimage. 2005;24(1):12-20.

doi:10.1016/j.neuroimage.2004.06.046.

72. Rauch PJ, Park HS, Knisely JPS, Chiang VL, Vortmeyer AO. Delayed radiation-induced vasculitic leukoencephalopathy. Int J Radiat Oncol Biol Phys. 2012;83(1):369-375. doi:10.1016/j.jjrobp.2011.06.1982.

73. Oh BC, Pagnini PG, Wang MY, et al. Stereotactic radiosurgery: Adjacent tissue injury and response after high-dose single fraction radiation: Part I - Histology, imaging, and molecular events. Neurosurgery. 2007;60(1):31-44. doi:10.1227/01.NEU.0000249191.23162.D2.

74. O'Reilly M a., Hynynen K. Blood-Brain Barrier: Real-time Feedback-controlled Focused Ultrasound Disruption by Using an Acoustic Emissions-based Controller. Radiology. 2012;263(1):96-106. doi:10.1148/radiol.11111417.

75. Tung Y-S, Vlachos F, Choi JJ, Deffieux T, Selert K, Konofagou EE. In vivo transcranial cavitation threshold detection during ultrasound-induced blood-brain barrier opening in mice. Phys Med Biol. 2010;55(20):6141-6155. doi:10.1088/0031-9155/55/20/007.

76. Samuel S, Cooper M a, Bull JL, Fowlkes JB, Miller DL. An ex vivo study of the correlation between acoustic emission and microvascular damage. Ultrasound Med Biol. 2009;35(9):1574-1586. doi:10.1016/j.ultrasmedbio.2009.04.013.

77. Madanshetty SI, Roy RA, Apfel RE. Acoustic microcavitation: its active and passive acoustic detection. J Acoust Soc Am. 1991;90(3):1515-1526.

78. Ilyichev VI, Koretz VL, Melnikov NP. Spectral characteristics of acoustic cavitation. Ultrasonics. 1989;27(6):357-361. doi:10.1016/0041-624X(89)90034-6.

79. Leighton TG. The Acoustic Bubble. J Acoust Soc Am. 1994;96(4):2616. doi:10.1121/1.410082.

80. Arvanitis CD, Livingstone MS, Vykhodtseva N, McDannold N. Controlled UltrasoundInduced Blood-Brain Barrier Disruption Using Passive Acoustic Emissions Monitoring. PLoS One. 2012;7(9). doi:10.1371/journal.pone.0045783.

81. Wu SY, Tung YS, Marquet F, et al. Transcranial cavitation detection in primates during blood-brain barrier opening-a performance assessment study. IEEE Trans Ultrason Ferroelectr Freq Control. 2014;61(6):966-978. doi:10.1109/TUFFC.2014.2992.

82. Chai WY, Chu PC, Tsai MY, et al. Magnetic-resonance imaging for kinetic analysis of permeability changes during focused ultrasound-induced blood-brain barrier opening and brain drug delivery. J Control Release. 2014;192:1-9. doi:10.1016/j.jconrel.2014.06.023.

83. Jordão JF, Thévenot E, Markham-Coultes $\mathrm{K}$, et al. Amyloid- $\beta$ plaque reduction, endogenous antibody delivery and glial activation by brain-targeted, transcranial focused 
ultrasound. Exp Neurol. 2013;248:16-29. doi:10.1016/j.expneurol.2013.05.008.

84. Leinenga G, Götz J. Scanning ultrasound removes amyloid-b and restores memory in an Alzheimer' s disease mouse model. Sci Transl Med. 2015;7(278).

85. Burke CW, Klibanov AL, Sheehan JP, Price RJ. Inhibition of glioma growth by microbubble activation in a subcutaneous model using low duty cycle ultrasound without significant heating. J Neurosurg. 2011;114(6):1654-1661.

doi:10.3171/2010.11.JNS101201.

86. Leinenga G, Gotz J. Scanning ultrasound removes amyloid- and restores memory in an Alzheimer's disease mouse model. Sci Transl Med. 2015;7(278):278ra33-278ra33. doi:10.1126/scitranslmed.aaa2512.

87. Scarcelli T, Jordão JF, O'Reilly M a, Ellens N, Hynynen K, Aubert I. Stimulation of hippocampal neurogenesis by transcranial focused ultrasound and microbubbles in adult mice. Brain Stimul. 2014;7(2):304-307. doi:10.1016/j.brs.2013.12.012.

88. Tufail $\mathrm{Y}$, Matyushov A, Baldwin N, et al. Transcranial pulsed ultrasound stimulates intact brain circuits. Neuron. 2010;66(5):681-694. doi:10.1016/j.neuron.2010.05.008.

89. Jalali S, Huang Y, Dumont DJ, Hynynen K. Focused ultrasound-mediated bbb disruption is associated with an increase in activation of AKT: experimental study in rats. BMC Neurol. 2010;10(1):114. doi:10.1186/1471-2377-10-114.

90. Tsai S-J. Transcranial focused ultrasound as a possible treatment for major depression. Med Hypotheses. 2015;84(4):381-383. doi:10.1016/j.mehy.2015.01.030.

91. Tyler WJ, Tufail Y, Finsterwald M, Tauchmann ML, Olson EJ, Majestic C. Remote excitation of neuronal circuits using low-intensity, low-frequency ultrasound. PLoS One. 2008;3(10):e3511. doi:10.1371/journal. pone.0003511.

92. Deffieux T, Younan Y, Wattiez N, Tanter M, Pouget P, Aubry J-F. Low-intensity focused ultrasound modulates monkey visuomotor behavior. Curr Biol. 2013;23(23):2430-2433. doi:10.1016/j.cub.2013.10.029.

93. Kim H, Taghados SJ, Fischer K, Maeng LS, Park S, Yoo SS. Noninvasive Transcranial Stimulation of Rat Abducens Nerve by Focused Ultrasound. Ultrasound Med Biol. 2012;38(9):1568-1575. doi:10.1016/j.ultrasmedbio.2012.04.023.

94. Yoo SS, Bystritsky A, Lee JH, et al. Focused ultrasound modulates region-specific brain activity. Neuroimage. 2011;56(3):1267-1275. doi:10.1016/j.neuroimage.2011.02.058.

95. Legon W, Rowlands A, Opitz A, Sato TF, Tyler WJ. Pulsed Ultrasound Differentially Stimulates Somatosensory Circuits in Humans as Indicated by EEG and fMRI. PLoS One. 2012;7(12). doi:10.1371/journal.pone.0051177.

96. Lee W, Kim H, Jung Y, Song I-U, Chung YA, Yoo S-S. Image-guided transcranial focused ultrasound stimulates human primary somatosensory cortex. Sci Rep. 2015;5:8743. doi:10.1038/srep08743.

97. Liu H-L, Huang C-Y, Chen J-Y, Wang H-YJ, Chen P-Y, Wei K-C. Pharmacodynamic and therapeutic investigation of focused ultrasound-induced blood-brain barrier opening for enhanced temozolomide delivery in glioma treatment. PLoS One. 2014;9(12):e114311. 
doi:10.1371/journal.pone.0114311.

98. Treat LH, McDannold N, Vykhodtseva N, Zhang Y, Tam K, Hynynen K. Targeted delivery of doxorubicin to the rat brain at therapeutic levels using MRI-guided focused ultrasound. Int J Cancer. 2007;121(4):901-907. doi:10.1002/ijc.22732.

99. Jordao JF, Ayala-Grosso C a, Markham K, et al. Antibodies targeted to the brain with image-guided focused ultrasound reduces amyloid-beta plaque load in the TgCRND8 mouse model of Alzheimer's disease. PLoS One. 2010;5(5):e10549.

doi:10.1371/journal.pone.0010549.

100. Samiotaki G, Acosta C, Wang S, Konofagou EE. Enhanced delivery and bioactivity of the neurturin neurotrophic factor through focused ultrasound-mediated blood-brain barrier opening in vivo. J Cereb Blood Flow Metab. 2015;35:611-622.

doi:10.1038/jcbfm.2014.236.

101. Aryal M, Park J, Vykhodtseva N, Zhang Y-Z, McDannold N. Enhancement in blood-tumor barrier permeability and delivery of liposomal doxorubicin using focused ultrasound and microbubbles: evaluation during tumor progression in a rat glioma model. Phys Med Biol. 2015;60(6):2511-2527. doi:10.1088/0031-9155/60/6/2511.

102. Fan $\mathrm{C}-\mathrm{H}$, Ting $\mathrm{C}-\mathrm{Y}$, Liu H-L, et al. Antiangiogenic-targeting drug-loaded microbubbles combined with focused ultrasound for glioma treatment. Biomaterials. 2013;34(8):21422155. doi:10.1016/j.biomaterials.2012.11.048.

103. Yang F-Y, Wong T-T, Teng M-C, et al. Focused ultrasound and interleukin-4 receptortargeted liposomal doxorubicin for enhanced targeted drug delivery and antitumor effect in glioblastoma multiforme. J Control Release. 2012;160(3):652-658. doi:10.1016/j.jconrel.2012.02.023.

104. Alkins RD, Brodersen PM, Sodhi RNS, Hynynen K. Enhancing drug delivery for boron neutron capture therapy of brain tumors with focused ultrasound. Neuro Oncol. 2013;15(9):1225-1235. doi:10.1093/neuonc/not052.

105. Malmström A, Grønberg BH, Marosi C, et al. Temozolomide versus standard 6-week radiotherapy versus hypofractionated radiotherapy in patients older than 60 years with glioblastoma: the Nordic randomised, phase 3 trial. Lancet Oncol. 2012;13(9):916-926. doi:10.1016/S1470-2045(12)70265-6.

106. Darlix A, Baumann C, Lorgis V, et al. Prolonged administration of adjuvant temozolomide improves survival in adult patients with glioblastoma. Anticancer Res. 2013;33(8):34673474.

107. Gilbert MR, Wang M, Aldape KD, et al. Dose-dense temozolomide for newly diagnosed glioblastoma: a randomized phase III clinical trial. J Clin Oncol. 2013;31(32):4085-4091. doi:10.1200/JCO.2013.49.6968.

108. Norden AD, Lesser GJ, Drappatz J, et al. Phase 2 study of dose-intense temozolomide in recurrent glioblastoma. Neuro Oncol. 2013;15(7):930-935. doi:10.1093/neuonc/not040.

109. Wei K-C, Chu P-C, Wang H-YJ, et al. Focused ultrasound-induced blood-brain barrier opening to enhance temozolomide delivery for glioblastoma treatment: a preclinical study. PLoS One. 2013;8(3):e58995. doi:10.1371/journal.pone.0058995. 
110. Westphal M, Hilt DC, Bortey E, et al. A phase 3 trial of local chemotherapy with biodegradable carmustine (BCNU) wafers (Gliadel wafers) in patients with primary malignant glioma. Neuro Oncol. 2003;5(2):79-88. doi:10.1215/S1522-8517-02-00023-6.

111. Gururangan S, Cokgor L, Rich JN, et al. Phase I study of Gliadel wafers plus temozolomide in adults with recurrent supratentorial high-grade gliomas. Neuro Oncol. 2001;3(4):246-250.

112. Westphal M, Ram Z, Riddle V, Hilt D, Bortey E. Gliadel wafer in initial surgery for malignant glioma: long-term follow-up of a multicenter controlled trial. Acta Neurochir (Wien). 2006;148(3):269-75; discussion 275. doi:10.1007/s00701-005-0707-z.

113. Attenello FJ, Mukherjee D, Datoo G, et al. Use of Gliadel (BCNU) wafer in the surgical treatment of malignant glioma: a 10-year institutional experience. Ann Surg Oncol. 2008;15(10):2887-2893. doi:10.1245/s10434-008-0048-2.

114. Treatment G, Hua M-Y, Liu H-L, et al. Blood-brain barrier disruption with focused ultrasound enhances delivery of chemotherapeutic drugs for glioblastoma treatment. Radiology. 2010;255(2):415-425. doi:10.1148/radiol.10090699.

115. Hau P, Fabel K, Baumgart $U$, et al. Pegylated liposomal doxorubicin-efficacy in patients with recurrent high-grade glioma. Cancer. 2004;100(6):1199-1207. doi:10.1002/cncr.20073.

116. Fabel K, Dietrich J, Hau $\mathrm{P}$, et al. Long-term stabilization in patients with malignant glioma after treatment with liposomal doxorubicin. Cancer. 2001;92(7):1936-1942.

117. Ananda S, Nowak AK, Cher L, et al. Phase 2 trial of temozolomide and pegylated liposomal doxorubicin in the treatment of patients with glioblastoma multiforme following concurrent radiotherapy and chemotherapy. J Clin Neurosci. 2011;18(11):1444-1448. doi:10.1016/j.jocn.2011.02.026.

118. Savolainen S, Kortesniemi M, Timonen M, et al. Boron neutron capture therapy (BNCT) in Finland: technological and physical prospects after 20 years of experiences. Phys Med. 2013;29(3):233-248. doi:10.1016/j.ejmp.2012.04.008.

119. Kankaanranta L, Seppälä $T$, Koivunoro H, et al. Boron neutron capture therapy in the treatment of locally recurred head-and-neck cancer: final analysis of a phase I/II trial. Int J Radiat Oncol Biol Phys. 2012;82(1):e67-75. doi:10.1016/j.jrobp.2010.09.057.

120. Miyatake S-I, Kawabata S, Yokoyama K, et al. Survival benefit of boron neutron capture therapy for recurrent malignant gliomas. Appl Radiat Isot. 2009;67(7-8 Suppl):S22-4. doi:10.1016/j.apradiso.2009.03.032.

121. Hopewell JW, Gorlia T, Pellettieri L, Giusti V, H-Stenstam B, Sköld K. Boron neutron capture therapy for newly diagnosed glioblastoma multiforme: an assessment of clinical potential. Appl Radiat Isot. 2011;69(12):1737-1740. doi:10.1016/j.apradiso.2011.03.022.

122. Yang F-Y, Chen Y-W, Chou F-I, Yen S-H, Lin Y-L, Wong T-T. Boron neutron capture therapy for glioblastoma multiforme: enhanced drug delivery and antitumor effect following blood-brain barrier disruption induced by focused ultrasound. Future Oncol. 2012;8(10):1361-1369. doi:10.2217/fon.12.118. 
123. Yang F-Y, Lin Y-L, Chou F-I, et al. Pharmacokinetics of BPA in Gliomas with Ultrasound Induced Blood-Brain Barrier Disruption as Measured by Microdialysis. PLoS One. 2014;9(6):e100104. doi:10.1371/journal.pone.0100104.

124. Kawalec P, Łopuch S, Mikrut A. Effectiveness of Targeted Therapy in Patients With Previously Untreated Metastatic Breast Cancer: A Systematic Review and Meta-Analysis. Clin Breast Cancer. 2015;15(2):90-100.e1. doi:10.1016/j.clbc.2014.10.006.

125. Spencer B, Masliah E. Immunotherapy for Alzheimer's disease: past, present and future. Front Aging Neurosci. 2014;6:114. doi:10.3389/fnagi.2014.00114.

126. Sheikov N, McDannold N, Vykhodtseva N, Jolesz F, Hynynen K. Cellular mechanisms of the blood-brain barrier opening induced by ultrasound in presence of microbubbles. Ultrasound Med Biol. 2004;30(7):979-989. doi:10.1016/j.ultrasmedbio.2004.04.010.

127. Kinoshita M, McDannold N, Jolesz FA, Hynynen K. Targeted delivery of antibodies through the blood-brain barrier by MRI-guided focused ultrasound. Biochem Biophys Res Commun. 2006;340(4):1085-1090. doi:10.1016/j.bbrc.2005.12.112.

128. Raymond SB, Treat LH, Dewey JD, McDannold NJ, Hynynen K, Bacskai BJ. Ultrasound enhanced delivery of molecular imaging and therapeutic agents in Alzheimer's disease mouse models. PLoS One. 2008;3(5):e2175. doi:10.1371/journal.pone.0002175.

129. Kinoshita M, McDannold N, Jolesz FA, Hynynen K. Noninvasive localized delivery of Herceptin to the mouse brain by MRI-guided focused ultrasound-induced blood-brain barrier disruption. Proc Natl Acad Sci U S A. 2006;103(31):11719-11723. doi:10.1073/pnas.0604318103.

130. Ahmed AO, Mantini AM, Fridberg DJ, Buckley PF. Brain-derived neurotrophic factor (BDNF) and neurocognitive deficits in people with schizophrenia: A meta-analysis. Psychiatry Res. 2015;226(1):1-13. doi:10.1016/j.psychres.2014.12.069.

131. Kalia M, Costa E Silva J. Biomarkers of psychiatric diseases: current status and future prospects. Metabolism. 2015;64(3 Suppl 1):S11-5. doi:10.1016/j.metabol.2014.10.026.

132. Theoharides TC, Athanassiou M, Panagiotidou S, Doyle R. Dysregulated brain immunity and neurotrophin signaling in Rett syndrome and autism spectrum disorders. $J$ Neuroimmunol. 2015;279:33-38. doi:10.1016/j.jneuroim.2014.12.003.

133. Hegarty S V, O'Keeffe GW, Sullivan AM. Neurotrophic factors: from neurodevelopmental regulators to novel therapies for Parkinson's disease. Neural Regen Res. 2014;9(19):1708-1711. doi:10.4103/1673-5374.143410.

134. Baseri B, Choi JJ, Deffieux T, et al. Activation of signaling pathways following localized delivery of systemically administered neurotrophic factors across the blood-brain barrier using focused ultrasound and microbubbles. Phys Med Biol. 2012;57(7):N65-81. doi:10.1088/0031-9155/57/7/N65.

135. Wang F, Shi Y, Lu L, et al. Targeted delivery of GDNF through the blood-brain barrier by MRI-guided focused ultrasound. PLoS One. 2012;7(12):e52925. doi:10.1371/journal.pone.0052925.

136. Thomas AA, Brennan CW, DeAngelis LM, Omuro AM. Emerging therapies for 
glioblastoma. JAMA Neurol. 2014;71(11):1437-1444. doi:10.1001/jamaneurol.2014.1701.

137. Ampie L, Woolf EC, Dardis C. Immunotherapeutic advancements for glioblastoma. Front Oncol. 2015;5:12. doi:10.3389/fonc.2015.00012.

138. Chen P-Y, Hsieh H-Y, Huang C-Y, Lin C-Y, Wei K-C, Liu H-L. Focused ultrasoundinduced blood-brain barrier opening to enhance interleukin-12 delivery for brain tumor immunotherapy: a preclinical feasibility study. J Transl Med. 2015;13:451.

doi:10.1186/s12967-015-0451-y.

139. Bozzuto G, Molinari A. Liposomes as nanomedical devices. Int J Nanomedicine. 2015;10:975-999. doi:10.2147/IJN.S68861.

140. Mohan A, Narayanan S, Sethuraman S, Krishnan UM. Novel resveratrol and 5fluorouracil coencapsulated in PEGylated nanoliposomes improve chemotherapeutic efficacy of combination against head and neck squamous cell carcinoma. Biomed Res Int. 2014;2014:424239. doi:10.1155/2014/424239.

141. Immordino ML, Dosio F, Cattel L. Stealth liposomes: review of the basic science, rationale, and clinical applications, existing and potential. Int $\mathrm{J}$ Nanomedicine. 2006;1(3):297-315.

142. Dicheva BM, Koning GA. Targeted thermosensitive liposomes: an attractive novel approach for increased drug delivery to solid tumors. Expert Opin Drug Deliv. 2014;11(1):83-100. doi:10.1517/17425247.2014.866650.

143. Storm G, Roerdink FH, Steerenberg PA, de Jong WH, Crommelin DJ. Influence of lipid composition on the antitumor activity exerted by doxorubicin-containing liposomes in a rat solid tumor model. Cancer Res. 1987;47(13):3366-3372.

144. Yang F-Y, Wang H-E, Liu R-S, et al. Pharmacokinetic Analysis of 111In-Labeled Liposomal Doxorubicin in Murine Glioblastoma after Blood-Brain Barrier Disruption by Focused Ultrasound. Heymann D, ed. PLoS One. 2012;7(9):e45468. doi:10.1371/journal.pone.0045468.

145. Aryal M, Vykhodtseva N, Zhang Y-Z, McDannold N. Multiple sessions of liposomal doxorubicin delivery via focused ultrasound mediated blood-brain barrier disruption: A safety study. J Control Release. 2015;204:60-69. doi:10.1016/j.jconrel.2015.02.033.

146. Nomikou N, Tiwari P, Trehan T, Gulati K, McHale AP. Studies on neutral, cationic and biotinylated cationic microbubbles in enhancing ultrasound-mediated gene delivery in vitro and in vivo. Acta Biomater. 2012;8(3):1273-1280. doi:10.1016/j.actbio.2011.09.010.

147. Fan $\mathrm{C}-\mathrm{H}$, Liu $\mathrm{H}-\mathrm{L}$, Ting $\mathrm{C}-\mathrm{Y}$, et al. Submicron-bubble-enhanced focused ultrasound for blood-brain barrier disruption and improved CNS drug delivery. PLoS One. 2014;9(5):e96327. doi:10.1371/journal.pone.0096327.

148. Fan C-H, Ting C-Y, Chang Y-C, Wei K-C, Liu H-L, Yeh C-K. Drug-loaded bubbles with matched focused ultrasound excitation for concurrent blood-brain barrier opening and brain-tumor drug delivery. Acta Biomater. 2015;15:89-101. doi:10.1016/j.actbio.2014.12.026.

149. Ting $\mathrm{C}-\mathrm{Y}$, Fan $\mathrm{C}-\mathrm{H}$, Liu $\mathrm{H}-\mathrm{L}$, et al. Concurrent blood-brain barrier opening and local drug 
delivery using drug-carrying microbubbles and focused ultrasound for brain glioma treatment. Biomaterials. 2012;33(2):704-712. doi:10.1016/j.biomaterials.2011.09.096.

150. Fan $\mathrm{C}-\mathrm{H}$, Ting $\mathrm{C}-\mathrm{Y}$, Lin $\mathrm{H}-\mathrm{J}$, et al. SPIO-conjugated, doxorubicin-loaded microbubbles for concurrent MRI and focused-ultrasound enhanced brain-tumor drug delivery. Biomaterials. 2013;34(14):3706-3715. doi:10.1016/j.biomaterials.2013.01.099.

151. Boylan NJ, Suk JS, Lai SK, et al. Highly compacted DNA nanoparticles with low MW PEG coatings: in vitro, ex vivo and in vivo evaluation. J Control Release. 2012;157(1):72-79. doi:10.1016/j.jconrel.2011.08.031.

152. Suh J, Choy K-L, Lai SK, et al. PEGylation of nanoparticles improves their cytoplasmic transport. Int J Nanomedicine. 2007;2(4):735-741.

153. Tosi G, Bortot B, Ruozi B, et al. Potential use of polymeric nanoparticles for drug delivery across the blood-brain barrier. Curr Med Chem. 2013;20(17):2212-2225.

154. Giteau A, Venier-Julienne MC, Aubert-Pouëssel A, Benoit JP. How to achieve sustained and complete protein release from PLGA-based microparticles? Int J Pharm. 2008;350(12):14-26. doi:10.1016/j.ijpharm.2007.11.012.

155. Dufort S, Sancey L, Coll J-L. Physico-chemical parameters that govern nanoparticles fate also dictate rules for their molecular evolution. Adv Drug Deliv Rev. 2012;64(2):179-189. doi:10.1016/j.addr.2011.09.009.

156. Kumari A, Yadav SK, Yadav SC. Biodegradable polymeric nanoparticles based drug delivery systems. Colloids Surfaces B Biointerfaces. 2010;75(1):1-18. doi:10.1016/j.colsurfb.2009.09.001.

157. Chappell JC, Song J, Burke CW, Klibanov AL, Price RJ. Targeted delivery of nanoparticles bearing fibroblast growth factor-2 by ultrasonic microbubble destruction for therapeutic arteriogenesis. Small. 2008;4(10):1769-1777. doi:10.1002/smll.200800806.

158. Burke CW, Alexander E, Timbie K, Kilbanov AL, Price RJ. Ultrasound-activated agents comprised of 5FU-bearing nanoparticles bonded to microbubbles inhibit solid tumor growth and improve survival. Mol Ther. 2013;22(2):321-328. doi:10.1038/mt.2013.259.

159. Naumova A V, Modo M, Moore A, Murry CE, Frank J a. Clinical imaging in regenerative medicine. Nat Biotechnol. 2014;32(8):804-818. doi:10.1038/nbt.2993.

160. Chen F, Ehlerding EB, Cai W. Theranostic Nanoparticles. J Nucl Med. 2014;55(12):19191922. doi:10.2967/jnumed.114.146019.

161. Walia S, Acharya A. Silica micro/nanospheres for theranostics: from bimodal MRI and fluorescent imaging probes to cancer therapy. Beilstein J Nanotechnol. 2015;6:546-558. doi:10.3762/bjnano.6.57.

162. Mastorakos P, Zhang C, Berry S, et al. Highly PEGylated DNA Nanoparticles Provide Uniform and Widespread Gene Transfer in the Brain. Adv Healthc Mater. 2015:n/a--n/a. doi:10.1002/adhm.201400800.

163. Grigsby CL, Leong KW. Balancing protection and release of DNA: tools to address a bottleneck of non-viral gene delivery. J R Soc Interface. 2010;7 Suppl 1(September 2009):S67-82. doi:10.1098/rsif.2009.0260. 
164. Burke CW, Suk JS, Kim AJ, et al. Markedly Enhanced Skeletal Muscle Transfection Achieved by the Ultrasound-Targeted Delivery of Non-Viral Gene Nanocarriers with Microbubbles. J Control Release. 2012;162(2):414-421. doi:10.1016/j.jconrel.2012.07.005.

165. Allard E, Passirani C, Benoit J-P. Convection-enhanced delivery of nanocarriers for the treatment of brain tumors. Biomaterials. 2009;30(12):2302-2318. doi:10.1016/j.biomaterials.2009.01.003.

166. Zimmermann DR, Dours-Zimmermann MT. Extracellular matrix of the central nervous system: From neglect to challenge. Histochem Cell Biol. 2008;130:635-653. doi:10.1007/s00418-008-0485-9.

167. Sykova E, Nicholson C, Syková E, Nicholson C. Diffusion in brain extracellular space. Physiol Rev. 2008;88(4):1277-1340. doi:10.1152/physrev.00027.2007.

168. Kenny GD, Bienemann AS, Tagalakis AD, et al. Multifunctional receptor-targeted nanocomplexes for the delivery of therapeutic nucleic acids to the Brain. Biomaterials. 2013;34(36):9190-9200. doi:10.1016/j.biomaterials.2013.07.081.

169. Nance EA, Woodworth GF, Sailor KA, et al. A dense poly(ethylene glycol) coating improves penetration of large polymeric nanoparticles within brain tissue. Sci Transl Med. 2012;4(149):149ra119. doi:10.1126/scitranslmed.3003594.

170. Netti P a, Berk D a, Swartz M a, Grodzinsky a J, Jain RK. Role of extracellular matrix assembly in interstitial transport in solid tumors. Cancer Res. 2000;60(9):2497-2503.

171. Heldin $\mathrm{C}-\mathrm{H}$, Rubin K, Pietras K, Ostman A. High interstitial fluid pressure - an obstacle in cancer therapy. Nat Rev Cancer. 2004;4(10):806-813. doi:10.1038/nrc1456.

172. Pluen a, Boucher Y, Ramanujan S, et al. Role of tumor-host interactions in interstitial diffusion of macromolecules: cranial vs. subcutaneous tumors. Proc Natl Acad Sci U S A. 2001;98(8):4628-4633. doi:10.1073/pnas.081626898.

173. Jain RK, Stylianopoulos T. Delivering nanomedicine to solid tumors. Nat Rev Clin Oncol. 2010;7(11):653-664. doi:10.1038/nrclinonc.2010.139.

174. Beyer I, Li Z, Persson J, et al. Controlled extracellular matrix degradation in breast cancer tumors improves therapy by trastuzumab. Mol Ther. 2011;19(3):479-489. doi:10.1038/mt.2010.256.

175. Stylianopoulos T, Poh MZ, Insin N, et al. Diffusion of particles in the extracellular matrix: The effect of repulsive electrostatic interactions. Biophys J. 2010;99(5):1342-1349. doi:10.1016/j.bpj.2010.06.016.

176. Thorne RG, Nicholson C. In vivo diffusion analysis with quantum dots and dextrans predicts the width of brain extracellular space. Proc Natl Acad Sci U S A. 2006;103(14):5567-5572. doi:10.1073/pnas.0509425103.

177. Nance E, Timbie K, Miller GW, et al. Non-invasive delivery of stealth, brain-penetrating nanoparticles across the blood-brain barrier using MRI-guided focused ultrasound. $J$ Control Release. 2014;189:123-132. doi:10.1016/j.jconrel.2014.06.031.

178. Nance E, Zhang C, Shih T-Y, Xu Q, Schuster BS, Hanes J. Brain-penetrating 
nanoparticles improve paclitaxel efficacy in malignant glioma following local administration. ACS Nano. 2014;8(10):10655-10664. doi:10.1021/nn504210g.

179. Gref R, Minamitake Y, Peracchia MT, Trubetskoy V, Torchilin V, Langer R. Biodegradable long-circulating polymeric nanospheres. Science. 1994;263(5153):16001603. doi:10.1126/science.8128245.

180. Calvo $\mathrm{P}$, Gouritin $\mathrm{B}$, Chacun $\mathrm{H}$, et al. Long-circulating pegylated polycyanoacrylate nanoparticles as new drug carrier for brain delivery. Pharm Res. 2001;18(8):1157-1166. doi:10.1023/A:1010931127745.

181. Morizet J, Eve G, El H. Poly ( ethylene glycol ) -Coated Hexadecylcyanoacrylate Nanospheres Display a Combined Effect for Brain Tumor Targeting. J Pharmacol Exp Ther. 2002;303(3):928-936. doi:10.1124/jpet.102.039669.thelial.

182. Coley WB. The treatment of malignant tumors by repeated inoculations of erysipelas. With a report of ten original cases. 1893. Clin Orthop Relat Res. 1991;(262):3-11.

183. Wiemann B, Starnes CO. Coley's toxins, tumor necrosis factor and cancer research: a historical perspective. Pharmacol Ther. 1994;64(3):529-564.

184. BILLINGHAM RE, BRENT L, MEDAWAR PB. Actively acquired tolerance of foreign cells. Nature. 1953;172(4379):603-606.

185. BALDWIN RW. Immunity to methylcholanthrene-induced tumours in inbred rats following atrophy and regression of the implanted tumours. Br J Cancer. 1955;9(4):652-657.

186. FOLEY EJ. Antigenic properties of methylcholanthrene-induced tumors in mice of the strain of origin. Cancer Res. 1953;13(12):835-837.

187. Burnet FM. Immunological aspects of malignant disease. Lancet (London, England). 1967;1(7501):1171-1174.

188. Zinkernagel RM, Doherty PC. MHC-restricted cytotoxic T cells: studies on the biological role of polymorphic major transplantation antigens determining T-cell restrictionspecificity, function, and responsiveness. Adv Immunol. 1979;27:51-177.

189. Zinkernagel RM. Thymus and lymphohemopoietic cells: their role in T cell maturation in selection of T cells' $\mathrm{H}$-2-restriction-specificity and in $\mathrm{H}$-2 linked Ir gene control. Immunol Rev. 1978;42:224-270.

190. Boon $\mathrm{T}$, van der Bruggen $\mathrm{P}$. Human tumor antigens recognized by $\mathrm{T}$ lymphocytes. $\mathrm{J}$ Exp Med. 1996;183(3):725-729.

191. Rosenberg SA. A new era for cancer immunotherapy based on the genes that encode cancer antigens. Immunity. 1999;10(3):281-287.

192. Celluzzi CM, Mayordomo JI, Storkus WJ, Lotze MT, Falo LD. Peptide-pulsed dendritic cells induce antigen-specific CTL-mediated protective tumor immunity. J Exp Med. 1996;183(1):283-287.

193. Lanier LL. A renaissance for the tumor immunosurveillance hypothesis. Nat Med. 2001;7(11):1178-1180. doi:10.1038/nm1101-1178. 
194. Diamantopoulos P, Gogas H. Melanoma immunotherapy dominates the field. Ann Trans/ Med. 2016;4(14):269. doi:10.21037/atm.2016.06.32.

195. Michielin O, Hoeller C. Gaining momentum: New options and opportunities for the treatment of advanced melanoma. Cancer Treat Rev. June 2015. doi:10.1016/j.ctrv.2015.05.012.

196. Immunotherapy: combine and conquer. Nat Med. 2015;21(5):415. doi:10.1038/nm.3865.

197. Guo Z, Tilburgs T, Wong B, Strominger JL. Dysfunction of dendritic cells in aged C57BL/6 mice leads to failure of natural killer cell activation and of tumor eradication. Proc Natl Acad Sci U S A. 2014;111(39):14199-14204. doi:10.1073/pnas.1414780111.

198. Guillerey C, Huntington ND, Smyth MJ. Targeting natural killer cells in cancer immunotherapy. Nat Immunol. 2016;17(9):1025-1036. doi:10.1038/ni.3518.

199. Colegio OR, Chu N-Q, Szabo AL, et al. Functional polarization of tumour-associated macrophages by tumour-derived lactic acid. Nature. 2014;513(7519):559-563. doi:10.1038/nature13490.

200. Shankaran V, Ikeda H, Bruce AT, et al. IFNgamma and lymphocytes prevent primary tumour development and shape tumour immunogenicity. Nature. 2001;410(6832):11071111. doi:10.1038/35074122.

201. Rock KL, Reits E, Neefjes J. Present Yourself! By MHC Class I and MHC Class II Molecules. Trends Immunol. 2016. doi:10.1016/j.it.2016.08.010.

202. Keir ME, Butte MJ, Freeman GJ, Sharpe AH. PD-1 and its ligands in tolerance and immunity. Annu Rev Immunol. 2008;26:677-704. doi:10.1146/annurev.immunol.26.021607.090331.

203. Yang W, Bai $Y$, Xiong $Y$, et al. Potentiating the antitumour response of CD8+ T cells by modulating cholesterol metabolism. Nature. March 2016. doi:10.1038/nature17412.

204. Shah $\mathrm{W}$, Yan $\mathrm{X}$, Jing $\mathrm{L}$, Zhou $\mathrm{Y}$, Chen $\mathrm{H}$, Wang $\mathrm{Y}$. A reversed CD4/CD8 ratio of tumorinfiltrating lymphocytes and a high percentage of CD4(+)FOXP3(+) regulatory $T$ cells are significantly associated with clinical outcome in squamous cell carcinoma of the cervix. Cell Mol Immunol. 2011;8(1):59-66. doi:10.1038/cmi.2010.56.

205. Zanetti M. Tapping CD4 T cells for cancer immunotherapy: the choice of personalized genomics. J Immunol. 2015;194(5):2049-2056. doi:10.4049/jimmunol.1402669.

206. Chaudhary B, Elkord E. Regulatory T Cells in the Tumor Microenvironment and Cancer Progression: Role and Therapeutic Targeting. Vaccines. 2016;4(3):28. doi:10.3390/vaccines4030028.

207. Preston CC, Maurer MJ, Oberg AL, et al. The Ratios of CD8+ T Cells to CD4+CD25+ FOXP3+ and FOXP3- T Cells Correlate with Poor Clinical Outcome in Human Serous Ovarian Cancer. Moser M, ed. PLoS One. 2013;8(11):e80063. doi:10.1371/journal.pone.0080063.

208. Gras Navarro A, Björklund AT, Chekenya M. Therapeutic potential and challenges of natural killer cells in treatment of solid tumors. Front Immunol. 2015;6:202. doi:10.3389/fimmu.2015.00202. 
209. Rhee I. Diverse macrophages polarization in tumor microenvironment. Arch Pharm Res. August 2016. doi:10.1007/s12272-016-0820-y.

210. Qian B-Z, Pollard JW. Macrophage diversity enhances tumor progression and metastasis. Cell. 2010;141(1):39-51. doi:10.1016/j.cell.2010.03.014.

211. Kim R, Emi M, Tanabe K. Cancer immunoediting from immune surveillance to immune escape. Immunology. 2007;121(1):1-14. doi:10.1111/j.1365-2567.2007.02587.x.

212. Dunn GP, Old LJ, Schreiber RD. The Three Es of Cancer Immunoediting. Annu Rev Immunol. 2004;22(1):329-360. doi:10.1146/annurev.immunol.22.012703.104803.

213. Schreiber RD, Old LJ, Smyth MJ. Cancer Immunoediting: Integrating Immunity's Roles in Cancer Suppression and Promotion. Science (80- ). 2011;331(6024):1565-1570. doi:10.1126/science.1203486.

214. Beatty GL, Gladney WL. Immune Escape Mechanisms as a Guide for Cancer Immunotherapy. Clin Cancer Res. 2015;21(4).

215. VERBIK D, JOSHI S. IMMUNE CELLS AND CYTOKINES - THEIR ROLE IN CANCERIMMUNOTHERAPY (REVIEW). Int J Oncol. August 1995. doi:10.3892/ijo.7.2.205.

216. Wherry EJ. T cell exhaustion. Nat Immunol. 2011;131(6):492-499. doi:10.1038/ni.2035.

217. Martinez GJ, Pereira RM, Äijö T, et al. The transcription factor NFAT promotes exhaustion of activated CD8 ${ }^{+}$T cells. Immunity. 2015;42(2):265-278.

doi:10.1016/j.immuni.2015.01.006.

218. Liu J, Zhang S, Hu Y, et al. Targeting PD-1 and Tim-3 Pathways to Reverse CD8 T-Cell Exhaustion and Enhance Ex Vivo T-Cell Responses to Autologous Dendritic/Tumor Vaccines. J Immunother. 2016;39(4):171-180. doi:10.1097/CJI.0000000000000122.

219. Mahoney KM, Freeman GJ, McDermott DF. The Next Immune-Checkpoint Inhibitors: PD1/PD-L1 Blockade in Melanoma. Clin Ther. March 2015. doi:10.1016/j.clinthera.2015.02.018.

220. Corso CD, Bindra RS. Success and failures of combined modalities in GBM: Old Problems and New Directions. Semin Radiat Oncol. 2016. doi:10.1016/j.semradonc.2016.06.003.

221. Sharma $P$, Allison JP. The future of immune checkpoint therapy. Science (80- ). 2015;348(6230):56-61. doi:10.1126/science.aaa8172.

222. Ott PA, Hodi FS, Robert C. CTLA-4 and PD-1/PD-L1 blockade: new immunotherapeutic modalities with durable clinical benefit in melanoma patients. Clin Cancer Res. 2013;19(19):5300-5309. doi:10.1158/1078-0432.CCR-13-0143.

223. Yun S, Vincelette ND, Green MR, Wahner Hendrickson AE, Abraham I. Targeting immune checkpoints in unresectable metastatic cutaneous melanoma: a systematic review and meta-analysis of anti-CTLA-4 and anti-PD-1 agents trials. Cancer Med. 2016;5(7):1481-1491. doi:10.1002/cam4.732.

224. Sharon E, Streicher H, Goncalves $P$, Chen HX. Immune checkpoint inhibitors in clinical trials. Chin J Cancer. 2014;33(9):434-444. doi:10.5732/cjc.014.10122. 
225. Chen DS, Irving BA, Hodi FS. Molecular pathways: next-generation immunotherapy-inhibiting programmed death-ligand 1 and programmed death-1. Clin Cancer Res. 2012;18(24):6580-6587. doi:10.1158/1078-0432.CCR-12-1362.

226. Peng W, Liu C, Xu C, et al. PD-1 blockade enhances T-cell migration to tumors by elevating IFN-y inducible chemokines. Cancer Res. 2012;72(20):5209-5218. doi:10.1158/0008-5472.CAN-12-1187.

227. Ahmadzadeh M, Johnson LA, Heemskerk B, et al. Tumor antigen-specific CD8 T cells infiltrating the tumor express high levels of PD-1 and are functionally impaired. Blood. 2009;114:1537-1544. doi:10.1182/blood-2008.

228. Brahmer JR, Hammers H, Lipson EJ. Nivolumab: targeting PD-1 to bolster antitumor immunity. Future Oncol. 2015;11(9):1307-1326. doi:10.2217/fon.15.52.

229. Brahmer JR, Tykodi SS, Chow LQM, et al. Safety and activity of anti-PD-L1 antibody in patients with advanced cancer. N Engl J Med. 2012;366(26):2455-2465.

doi:10.1056/NEJMoa1200694.

230. Topalian SL, Hodi FS, Brahmer JR, et al. Safety, activity, and immune correlates of antiPD-1 antibody in cancer. N Engl J Med. 2012;366(26):2443-2454.

doi:10.1056/NEJMoa1200690.

231. Koyama S, Akbay EA, Li YY, et al. Adaptive resistance to therapeutic PD-1 blockade is associated with upregulation of alternative immune checkpoints. Nat Commun. 2016;7:10501. doi:10.1038/ncomms10501.

232. Sanlorenzo M, Vujic I, Posch C, et al. Melanoma immunotherapy. Cancer Biol Ther. 2014;15(6).

233. Curran MA, Montalvo W, Yagita H, Allison JP. PD-1 and CTLA-4 combination blockade expands infiltrating $T$ cells and reduces regulatory $T$ and myeloid cells within B16 melanoma tumors. Proc Natl Acad Sci U S A. 2010;107(9):4275-4280. doi:10.1073/pnas.0915174107.

234. Duraiswamy J, Kaluza KM, Freeman GJ, et al. Dual blockade of PD-1 and CTLA-4 combined with tumor vaccine effectively restores $\mathrm{T}$-cell rejection function in tumors. Cancer Res. 2013;73(12):3591-3603. doi:10.1158/0008-5472.CAN-12-4100.

235. Seifert AM, Zeng S, Zhang JQ, et al. PD-1/PD-L1 blockade enhances T cell activity and antitumor efficacy of imatinib in gastrointestinal stromal tumors. Clin Cancer Res. July 2016. doi:10.1158/1078-0432.CCR-16-1163.

236. Kim JE, Patel MA, Mangraviti A, et al. Combination therapy with anti-PD-1, anti-TIM-3, and focal radiation results in regression of murine gliomas. Clin Cancer Res. June 2016. doi:10.1158/1078-0432.CCR-15-1535.

237. Dai M, Yip YY, Hellstrom I, Hellstrom KE. Curing mice with large tumors by locally delivering combinations of immunomodulatory antibodies HHS Public Access. Clin Cancer Res. 2014;1(215):1127-1138. doi:10.1158/1078-0432.CCR-14-1339.

238. Reardon DA, Gokhale PC, Klein SR, et al. Glioblastoma Eradication Following Immune Checkpoint Blockade in an Orthotopic, Immunocompetent Model. Cancer Immunol Res. 
2015;4(2):124-135. doi:10.1158/2326-6066.CIR-15-0151.

239. Shah $W$, Yan $X$, Jing $L$, Zhou $Y$, Chen $H$, Wang $Y$. A reversed CD4/CD8 ratio of tumorinfiltrating lymphocytes and a high percentage of CD4+FOXP3+ regulatory $T$ cells are significantly associated with clinical outcome in squamous cell carcinoma of the cervix. Cell Mol Immunol. 2010;8(1):59-66. doi:10.1038/cmi.2010.56.

240. Victor CT-S, Rech AJ, Maity A, et al. Radiation and dual checkpoint blockade activate non-redundant immune mechanisms in cancer. Nature. 2015;520(7547):373-377. doi:10.1038/nature14292.

241. North RJ. Gamma-irradiation facilitates the expression of adoptive immunity against established tumors by eliminating suppressor T cells. Cancer Immunol Immunother. 1984;16(3):175-181.

242. Levy A, Chargari C, Marabelle A, Perfettini J-L, Magné N, Deutsch E. Can immunostimulatory agents enhance the abscopal effect of radiotherapy? Eur J Cancer. 2016;62:36-45. doi:10.1016/j.ejca.2016.03.067.

243. Madersbacher S, Gröbl M, Kramer G, Dirnhofer S, Steiner GE, Marberger M. Regulation of heat shock protein 27 expression of prostatic cells in response to heat treatment. Prostate. 1998;37(3):174-181.

244. Kramer G, Steiner GE, Gröbl M, et al. Response to sublethal heat treatment of prostatic tumor cells and of prostatic tumor infiltrating T-cells. Prostate. 2004;58(2):109-120. doi:10.1002/pros.10314.

245. Wu F, Wang Z-B, Cao Y-D, et al. Expression of tumor antigens and heat-shock protein 70 in breast cancer cells after high-intensity focused ultrasound ablation. Ann Surg Oncol. 2007;14(3):1237-1242. doi:10.1245/s10434-006-9275-6.

246. Hu Z, Yang XY, Liu Y, et al. Release of endogenous danger signals from HIFU-treated tumor cells and their stimulatory effects on APCs. Biochem Biophys Res Commun. 2005;335(1):124-131. doi:10.1016/j.bbrc.2005.07.071.

247. Hundt W, O'Connell-Rodwell CE, Bednarski MD, Steinbach S, Guccione S. In vitro effect of focused ultrasound or thermal stress on HSP70 expression and cell viability in three tumor cell lines. Acad Radiol. 2007;14(7):859-870. doi:10.1016/j.acra.2007.04.008.

248. Kruse DE, Mackanos MA, O'Connell-Rodwell CE, Contag CH, Ferrara KW. Shortduration-focused ultrasound stimulation of Hsp70 expression in vivo. Phys Med Biol. 2008;53(13):3641-3660. doi:10.1088/0031-9155/53/13/017.

249. Borges TJ, Lang BJ, Lopes RL, Bonorino C. Modulation of Alloimmunity by Heat Shock Proteins. Front Immunol. 2016;7:303. doi:10.3389/fimmu.2016.00303.

250. Shevtsov M, Multhoff G. Heat Shock Protein-Peptide and HSP-Based Immunotherapies for the Treatment of Cancer. Front Immunol. 2016;7:171. doi:10.3389/fimmu.2016.00171.

251. Zhou P, Fu M, Bai J, Wang Z, Wu F. Immune response after high-intensity focused ultrasound ablation for H22 tumor. ASCO Meet Abstr. 2007;25(18_suppl):21169.

252. Deng J, Zhang Y, Feng J, Wu F. Dendritic cells loaded with ultrasound-ablated tumour induce in vivo specific antitumour immune responses. Ultrasound Med Biol. 
2010;36(3):441-448. doi:10.1016/j.ultrasmedbio.2009.12.004.

253. Xu Z-L, Zhu X-Q, Lu P, Zhou Q, Zhang J, Wu F. Activation of tumor-infiltrating antigen presenting cells by high intensity focused ultrasound ablation of human breast cancer. Ultrasound Med Biol. 2009;35(1):50-57. doi:10.1016/j.ultrasmedbio.2008.08.005.

254. Liu F, Hu Z, Qiu L, et al. Boosting high-intensity focused ultrasound-induced anti-tumor immunity using a sparse-scan strategy that can more effectively promote dendritic cell maturation. J Transl Med. 2010;8:7. doi:10.1186/1479-5876-8-7.

255. Yi Y, Han J, Zhao L, et al. Immune responses of dendritic cells combined with tumorderived autophagosome vaccine on hepatocellular carcinoma. Chin J Cancer Res. 2015;27(6):597-603. doi:10.3978/j.issn.1000-9604.2015.12.07.

256. Wang C, Sun W, Wright G, Wang AZ, Gu Z. Inflammation-Triggered Cancer Immunotherapy by Programmed Delivery of CpG and Anti-PD1 Antibody. Adv Mater. August 2016. doi:10.1002/adma.201506312.

257. Wu F, Zhou L, Chen WR. Host antitumour immune responses to HIFU ablation. Int J Hyperthermia. 2007;23(2):165-171.

258. Hu Z, Yang XY, Liu Y, et al. Investigation of HIFU-induced anti-tumor immunity in a murine tumor model. J Transl Med. 2007;5(1):34. doi:10.1186/1479-5876-5-34.

259. Xing $Y$, Lu X, Pua EC, Zhong $P$. The effect of high intensity focused ultrasound treatment on metastases in a murine melanoma model. Biochem Biophys Res Commun. 2008;375(4):645-650. doi:10.1016/j.bbrc.2008.08.072.

260. Lu P, Zhu X-Q, Xu Z-L, Zhou Q, Zhang J, Wu F. Increased infiltration of activated tumorinfiltrating lymphocytes after high intensity focused ultrasound ablation of human breast cancer. Surgery. 2009;145(3):286-293. doi:10.1016/j.surg.2008.10.010.

261. Zhang Y, Deng J, Feng J, Wu F. Enhancement of antitumor vaccine in ablated hepatocellular carcinoma by high-intensity focused ultrasound. World J Gastroenterol. 2010;16(28):3584-3591.

262. Xia J-Z, Xie F-L, Ran L-F, Xie X-P, Fan Y-M, Wu F. High-intensity focused ultrasound tumor ablation activates autologous tumor-specific cytotoxic T lymphocytes. Ultrasound Med Biol. 2012;38(8):1363-1371. doi:10.1016/j.ultrasmedbio.2012.03.009.

263. Huang X, Yuan F, Liang M, et al. M-HIFU inhibits tumor growth, suppresses STAT3 activity and enhances tumor specific immunity in a transplant tumor model of prostate cancer. PLoS One. 2012;7(7):e41632. doi:10.1371/journal.pone.0041632.

264. Wu F, Wang Z-B, Lu P, et al. Activated anti-tumor immunity in cancer patients after high intensity focused ultrasound ablation. Ultrasound Med Biol. 2004;30(9):1217-1222. doi:10.1016/j.ultrasmedbio.2004.08.003.

265. Unga J, Hashida M. Ultrasound induced cancer immunotherapy. Adv Drug Deliv Rev. 2014;72:144-153. doi:10.1016/j.addr.2014.03.004.

266. Alkins R, Burgess A, Ganguly $M$, et al. Focused ultrasound delivers targeted immune cells to metastatic brain tumors. Cancer Res. 2013;73(6):1892-1899. doi:10.1158/00085472. CAN-12-2609. 
267. Liu H-L, Hsieh H-Y, Lu L-A, Kang C-W, Wu M-F, Lin C-Y. Low-pressure pulsed focused ultrasound with microbubbles promotes an anticancer immunological response. J Transl Med. 2012;10(1):221. doi:10.1186/1479-5876-10-221.

268. Hunt SJ, Gade T, Soulen MC, Pickup S, Sehgal CM. Antivascular ultrasound therapy: magnetic resonance imaging validation and activation of the immune response in murine melanoma. J Ultrasound Med. 2015;34(2):275-287. doi:10.7863/ultra.34.2.275.

269. Yoo HS, Oh JE, Lee KH, Park TG. Biodegradable nanoparticles containing doxorubicinPLGA conjugate for sustained release. Pharm Res. 1999;16(7):1114-1118.

270. Timbie KF, Mead BP, Price RJ. Drug and gene delivery across the blood-brain barrier with focused ultrasound. J Control Release. September 2015. doi:10.1016/j.jconrel.2015.08.059.

271. Zhang P, Hu L, Yin Q, Zhang Z, Feng L, Li Y. Transferrin-conjugated polyphosphoester hybrid micelle loading paclitaxel for brain-targeting delivery: Synthesis, preparation and in vivo evaluation. J Control Release. January 2012. doi:10.1016/j.jconrel.2012.01.031.

272. Kreuter J. Mechanism of polymeric nanoparticle-based drug transport across the bloodbrain barrier (BBB). J Microencapsul. June 2012. doi:10.3109/02652048.2012.692491.

273. Gulyaev AE, Gelperina SE, Skidan IN, Antropov AS, Kivman GY, Kreuter J. Significant transport of doxorubicin into the brain with polysorbate 80-coated nanoparticles. Pharm Res. 1999;16(10):1564-1569.

274. Kulkarni SA, Feng SS. Effects of surface modification on delivery efficiency of biodegradable nanoparticles across the blood-brain barrier. Nanomedicine (Lond). 2011;6(2):377-394. doi:10.2217/nnm.10.131.

275. Shajahan AN, Wang A, Decker M, Minshall RD, Liu MC, Clarke R. Caveolin-1 tyrosine phosphorylation enhances paclitaxel-mediated cytotoxicity. J Biol Chem. 2007;282(8):5934-5943. doi:10.1074/jbc.M608857200.

276. Ambruosi A, Gelperina S, Khalansky A, Tanski S, Theisen A, Kreuter J. Influence of surfactants, polymer and doxorubicin loading on the anti-tumour effect of poly(butyl cyanoacrylate) nanoparticles in a rat glioma model. J Microencapsul. 2006;23(5):582592. doi:10.1080/02652040600788080.

277. Petri B, Bootz A, Khalansky A, et al. Chemotherapy of brain tumour using doxorubicin bound to surfactant-coated poly(butyl cyanoacrylate) nanoparticles: revisiting the role of surfactants. J Control Release. 2007;117(1):51-58. doi:10.1016/j.jconrel.2006.10.015.

278. Zhou J, Patel TR, Sirianni RW, et al. Highly penetrative, drug-loaded nanocarriers improve treatment of glioblastoma. Proc Natl Acad Sci U S A. 2013;110(29):1175111756. doi:10.1073/pnas.1304504110.

279. Diaz RJ, McVeigh PZ, O'Reilly MA, et al. Focused ultrasound delivery of Raman nanoparticles across the blood-brain barrier: Potential for targeting experimental brain tumors. Nanomedicine. December 2013. doi:10.1016/j.nano.2013.12.006.

280. Timbie K, Burke C, Nance E, et al. Ultrasound-targeted delivery of systemically administered therapeutic nanoparticles. J Acoust Soc Am. 2013;134(5):4047. 
doi:10.1121/1.4830769.

281. Konofagou EE. Optimization of the ultrasound-induced blood-brain barrier opening. Theranostics. 2012;2(12):1223-1237. doi:10.7150/thno.5576.

282. Guo X, Li Q, Zhang Z, Zhang D, Tu J. Investigation on the inertial cavitation threshold and shell properties of commercialized ultrasound contrast agent microbubbles. J Acoust Soc Am. 2013;134(2):1622-1631. doi:10.1121/1.4812887.

283. Fan Z, Chen D, Deng CX. Improving ultrasound gene transfection efficiency by controlling ultrasound excitation of microbubbles. J Control Release. 2013;170(3):401-413. doi:10.1016/j.jconrel.2013.05.039.

284. Gauthier M, King D, O'Brien W. Influence of microbubble size on postexcitation collapse thresholds for single ultrasound contrast agents using double passive cavitation detection. IEEE Trans Ultrason Ferroelectr Freq Control. 2013;60(5):877-879. doi:10.1109/TUFFC.2013.2644.

285. Hynynen K, McDannold N, Vykhodtseva N, Jolesz FA. Noninvasive MR imaging-guided focal opening of the blood-brain barrier in rabbits. Radiology. 2001;220(3):640-646. doi:10.1148/radiol.2202001804.

286. Choi JJ, Selert K, Vlachos F, Wong A, Konofagou EE. Noninvasive and localized neuronal delivery using short ultrasonic pulses and microbubbles. Proc Natl Acad Sci. 2011;108(40):16539-16544. doi:10.1073/pnas.1105116108.

287. Hoffmann A, Bredno J, Wendland M, Derugin N, Ohara P, Wintermark M. High and Low Molecular Weight Fluorescein Isothiocyanate (FITC)-Dextrans to Assess Blood-Brain Barrier Disruption: Technical Considerations. Trans/ Stroke Res. 2011;2(1):106-111. doi:10.1007/s12975-010-0049-x.

288. van Wamel A, Kooiman K, Emmer M, ten Cate FJ, Versluis M, de Jong N. Ultrasound microbubble induced endothelial cell permeability. J Control Release. 2006;116(2):e1002. doi:10.1016/j.jconrel.2006.09.071.

289. Wu S-Y, Chen CC, Tung Y-S, Olumolade OO, Konofagou EE. Effects of the microbubble shell physicochemical properties on ultrasound-mediated drug delivery to the brain. $J$ Control Release. June 2015. doi:10.1016/j.jconrel.2015.06.007.

290. Shang X, Wang P, Liu Y, Zhang Z, Xue Y. Mechanism of low-frequency ultrasound in opening blood-tumor barrier by tight junction. J Mol Neurosci. 2011;43(3):364-369. doi:10.1007/s12031-010-9451-9.

291. Burgess A, Nhan T, Moffatt C, Klibanov ALL, Hynynen K. Analysis of focused ultrasoundinduced blood-brain barrier permeability in a mouse model of Alzheimer's disease using two-photon microscopy. J Control Release. 2014;192:243-248. doi:10.1016/j.jconrel.2014.07.051.

292. Hersh DS, Kim AJ, Winkles JA, Eisenberg HM, Woodworth GF, Frenkel V. Emerging Applications of Therapeutic Ultrasound in Neuro-oncology: Moving Beyond Tumor Ablation. Neurosurgery. August 2016. doi:10.1227/NEU.0000000000001399.

293. Vykhodtseva N, McDannold N, Hynynen K. Progress and problems in the application of 
focused ultrasound for blood-brain barrier disruption. Ultrasonics. 2008;48(4):279-296. doi:10.1016/j.ultras.2008.04.004.

294. Meairs S. Facilitation of Drug Transport across the Blood-Brain Barrier with Ultrasound and Microbubbles. Pharmaceutics. 2015;7(3):275-293.

doi:10.3390/pharmaceutics7030275.

295. Mallick S, Benson R, Hakim A, Rath GK. Management of glioblastoma after recurrence: A changing paradigm. J Egypt Natl Canc Inst. 2016. doi:10.1016/j.jnci.2016.07.001.

296. Azad TD, Pan J, Connolly ID, Remington A, Wilson CM, Grant GA. Therapeutic strategies to improve drug delivery across the blood-brain barrier. Neurosurg Focus. 2015;38(3):E9. doi:10.3171/2014.12.FOCUS14758.

297. Ideguchi M, Kajiwara K, Goto $\mathrm{H}$, et al. MRI findings and pathological features in earlystage glioblastoma. J Neurooncol. 2015;123(2):289-297. doi:10.1007/s11060-015-1797y.

298. Hargrave DR, Zacharoulis S. Pediatric CNS tumors: current treatment and future directions. Expert Rev Neurother. 2007;7(8):1029-1042. doi:10.1586/14737175.7.8.1029.

299. Kim IH, Park C-K, Heo DS, et al. Radiotherapy followed by adjuvant temozolomide with or without neoadjuvant ACNU-CDDP chemotherapy in newly diagnosed glioblastomas: a prospective randomized controlled multicenter phase III trial. J Neurooncol. 2011;103(3):595-602. doi:10.1007/s11060-010-0427-y.

300. Silvani A, Gaviani P, Lamperti EA, et al. Cisplatinum and BCNU chemotherapy in primary glioblastoma patients. J Neurooncol. 2009;94(1):57-62. doi:10.1007/s11060-009-9800-0.

301. Cepeda V, Fuertes MA, Castilla J, Alonso C, Quevedo C, Pérez JM. Biochemical mechanisms of cisplatin cytotoxicity. Anticancer Agents Med Chem. 2007;7(1):3-18.

302. Huo T, Barth RF, Yang W, et al. Preparation, biodistribution and neurotoxicity of liposomal cisplatin following convection enhanced delivery in normal and F98 glioma bearing rats. PLoS One. 2012;7(11):e48752. doi:10.1371/journal.pone.0048752.

303. Sheleg S V, Korotkevich EA, Zhavrid EA, et al. Local chemotherapy with cisplatin-depot for glioblastoma multiforme. J Neurooncol. 2002;60(1):53-59.

304. Daneman R. The blood-brain barrier in health and disease. Ann Neurol. 2012;72(5):648672. doi:10.1002/ana.23648.

305. Krol S. Challenges in drug delivery to the brain: Nature is against us. $J$ Control Release. May 2012:1-11. doi:10.1016/j.jconrel.2012.04.044.

306. Drapeau A, Fortin D. Chemotherapy Delivery Strategies to the Central Nervous System: neither Optional nor Superfluous. Curr Cancer Drug Targets. June 2015.

307. Hendricks BK, Cohen-Gadol AA, Miller JC. Novel delivery methods bypassing the bloodbrain and blood-tumor barriers. Neurosurg Focus. 2015;38(3):E10. doi:10.3171/2015.1.FOCUS14767.

308. Gritsenko PG, Ilina O, Friedl P. Interstitial guidance of cancer invasion. J Pathol. 2012;226(2):185-199. doi:10.1002/path.3031. 
309. Zhao Y-Z, Chen L-J, Lin Q, et al. Using FUS induced BBB/BTB-opening technique combined with Doxorubicin liposomes to improve glioma-targeted inhibition. Oncotarget. September 2015. doi:10.18632/oncotarget.5144.

310. Frenkel V. Ultrasound mediated delivery of drugs and genes to solid tumors. Adv Drug Deliv Rev. 2008;60(10):1193-1208. doi:10.1016/j.addr.2008.03.007.

311. Kobus T, Vykhodtseva N, Pilatou M, Zhang Y, McDannold N. Safety Validation of Repeated Blood-Brain Barrier Disruption Using Focused Ultrasound. Ultrasound Med Biol. November 2015. doi:10.1016/j.ultrasmedbio.2015.10.009.

312. Barth RF, Kaur B. Rat brain tumor models in experimental neuro-oncology: the C6, 9L, T9, RG2, F98, BT4C, RT-2 and CNS-1 gliomas. J Neurooncol. 2009;94(3):299-312. doi:10.1007/s11060-009-9875-7.

313. Doblas $\mathrm{S}, \mathrm{He} \mathrm{T}$, Saunders $\mathrm{D}$, et al. In vivo characterization of several rodent glioma models by 1 H MRS. NMR Biomed. 2012;25(4):685-694. doi:10.1002/nbm.1785.

314. Doblas S, He T, Saunders D, et al. Glioma morphology and tumor-induced vascular alterations revealed in seven rodent glioma models by in vivo magnetic resonance imaging and angiography. J Magn Reson Imaging. 2010;32(2):267-275. doi:10.1002/jmri.22263.

315. Bencokova Z, Pauron L, Devic C, et al. Molecular and cellular response of the most extensively used rodent glioma models to radiation and/or cisplatin. J Neurooncol. 2008;86(1):13-21. doi:10.1007/s11060-007-9433-0.

316. Zhang C, Nance EA, Mastorakos P, et al. Convection Enhanced Delivery of CisplatinLoaded Brain Penetrating Nanoparticles Cures Malignant Glioma in Rats. J Control Release. 2016.

317. Prestayko A. Cisplatin: Current Status and New Developments. (Prestayko A, Crooke S, Carter S, eds.). Atlanta: Academic Press; 1979.

318. Rodriguez A, Tatter SB, Debinski W. Neurosurgical Techniques for Disruption of the Blood-Brain Barrier for Glioblastoma Treatment. Pharmaceutics. 2015;7(3):175-187. doi:10.3390/pharmaceutics7030175.

319. Voges J, Reszka R, Gossmann A, et al. Imaging-guided convection-enhanced delivery and gene therapy of glioblastoma. Ann Neurol. 2003;54(4):479-487. doi:10.1002/ana.10688.

320. Mackay JA, Deen DF, Szoka FC. Distribution in brain of liposomes after convection enhanced delivery; modulation by particle charge, particle diameter, and presence of steric coating. Brain Res. 2005;1035(2):139-153. doi:10.1016/j.brainres.2004.12.007.

321. Debinski W, Tatter SB. Convection-enhanced delivery for the treatment of brain tumors. Expert Rev Neurother. 2009;9(10):1519-1527. doi:10.1586/ern.09.99.

322. Goldwirt L, Canney M, Horodyckid C, et al. Enhanced brain distribution of carboplatin in a primate model after blood-brain barrier disruption using an implantable ultrasound device. Cancer Chemother Pharmacol. 2016;77(1):211-216. doi:10.1007/s00280-015-2930-5.

323. Stordal B, Pavlakis N, Davey R. Oxaliplatin for the treatment of cisplatin-resistant cancer: 
a systematic review. Cancer Treat Rev. 2007;33(4):347-357.

doi:10.1016/j.ctrv.2007.01.009.

324. Uchino $\mathrm{H}$, Matsumura $\mathrm{Y}$, Negishi $\mathrm{T}$, et al. Cisplatin-incorporating polymeric micelles (NC6004) can reduce nephrotoxicity and neurotoxicity of cisplatin in rats. $\mathrm{Br} \mathrm{J}$ Cancer. 2005;93(6):678-687. doi:10.1038/sj.bjc.6602772.

325. Dhar S, Kolishetti N, Lippard SJ, Farokhzad OC. Targeted delivery of a cisplatin prodrug for safer and more effective prostate cancer therapy in vivo. Proc Natl Acad Sci. 2011;108(5):1850-1855. doi:10.1073/pnas.1011379108.

326. Paraskar AS, Soni S, Chin KT, et al. Harnessing structure-activity relationship to engineer a cisplatin nanoparticle for enhanced antitumor efficacy. Proc Natl Acad Sci U S A. 2010;107(28):12435-12440. doi:10.1073/pnas.1007026107.

327. Plummer $\mathrm{R}$, Wilson $\mathrm{RH}$, Calvert $\mathrm{H}$, et al. A Phase I clinical study of cisplatin-incorporated polymeric micelles (NC-6004) in patients with solid tumours. Br J Cancer.

2011;104(4):593-598. doi:10.1038/bjc.2011.6.

328. Shahar T, Ram Z, Kanner A a. Convection-enhanced delivery catheter placements for high-grade gliomas: Complications and pitfalls. J Neurooncol. 2012;107(2):373-378. doi:10.1007/s11060-011-0751-x.

329. Park E-J, Zhang Y-Z, Vykhodtseva N, McDannold N. Ultrasound-mediated bloodbrain/blood-tumor barrier disruption improves outcomes with trastuzumab in a breast cancer brain metastasis model. J Control Release. 2012;163(3):277-284. doi:10.1016/j.jconrel.2012.09.007.

330. Stapleton S, Mirmilshteyn D, Zheng J, Allen C, Jaffray DA. Spatial Measurements of Perfusion, Interstitial Fluid Pressure and Liposomes Accumulation in Solid Tumors. $J$ Vis Exp. 2016;(114). doi:10.3791/54226.

331. Mekkaoui C, Metellus P, Kostis WJ, et al. Diffusion Tensor Imaging in Patients with Glioblastoma Multiforme Using the Supertoroidal Model. PLoS One.

2016;11(1):e0146693. doi:10.1371/journal.pone.0146693.

332. Belloli S, Brioschi A, Politi LS, et al. Characterization of biological features of a rat F98 GBM model: a PET-MRI study with [18F]FAZA and [18F]FDG. Nucl Med Biol. 2013;40(6):831-840. doi:10.1016/j.nucmedbio.2013.05.004.

333. Kobus T, Zervantonakis IK, Zhang Y, McDannold NJ. Growth inhibition in a brain metastasis model by antibody delivery using focused ultrasound-mediated blood-brain barrier disruption. J Control Release. August 2016. doi:10.1016/j.jconrel.2016.08.001.

334. Rizzitelli S, Giustetto P, Faletto D, Castelli DD, Aime S, Terreno E. The release of Doxorubicin from liposomes monitored by MRI and triggered by a combination of US stimuli led to a complete tumor regression in a breast cancer mouse model. J Control Release. 2016;230:57-63. doi:10.1016/j.jconrel.2016.03.040.

335. Yang F-Y, Chang W-Y, Lin W-T, et al. Focused ultrasound enhanced molecular imaging and gene therapy for multifusion reporter gene in glioma-bearing rat model. Oncotarget. 2015;6(34):36260-36268. doi:10.18632/oncotarget.5389. 
336. Rousseau J, Barth RF, Fernandez M, et al. Efficacy of intracerebral delivery of cisplatin in combination with photon irradiation for treatment of brain tumors. $J$ Neurooncol. 2010;98(3):287-295. doi:10.1007/s11060-009-0074-3.

337. Biston M-C, Joubert A, Adam J-F, et al. Cure of Fisher Rats Bearing Radioresistant F98 Glioma Treated with cis-Platinum and Irradiated with Monochromatic Synchrotron XRays. Cancer Res. 2004;64(7).

338. Chung $\mathrm{Y}-\mathrm{H}, \mathrm{Hsu} \mathrm{P}-\mathrm{H}$, Huang $\mathrm{C}-\mathrm{W}$, et al. Evaluation of prognostic integrin $\alpha 2 \beta 1 \mathrm{PET}$ tracer and concurrent targeting delivery using focused ultrasound for brain glioma detection. Mol Pharm. 2014;11(11):3904-3914. doi:10.1021/mp500296n.

339. Phase I/II study of NC-6004, a novel micellar formulation of cisplatin, in combination with gemcitabine in patients with pancreatic cancer in Asia -... | OncologyPRO. http://oncologypro.esmo.org/Meeting-Resources/ESMO-2012/Phase-I-II-study-of-NC6004-a-novel-micellar-formulation-of-cisplatin-in-combination-with-gemcitabine-inpatients-with-pancreatic-cancer-in-Asia-results-of-phase-I.

340. Alonso A. Ultrasound-induced blood-brain barrier opening for drug delivery. Front Neurol Neurosci. 2015;36:106-115. doi:10.1159/000366242.

341. Jahangiri A, Chin AT, Flanigan PM, Chen R, Bankiewicz K, Aghi MK. Convectionenhanced delivery in glioblastoma: a review of preclinical and clinical studies. $J$ Neurosurg. April 2016:1-10. doi:10.3171/2016.1.JNS151591.

342. Muldoon LL, Pagel MA, Netto JP, Neuwelt EA. Intra-arterial administration improves temozolomide delivery and efficacy in a model of intracerebral metastasis, but has unexpected brain toxicity. J Neurooncol. 2016;126(3):447-454. doi:10.1007/s11060-0152000-1.

343. Chen H, Chen CC, Acosta C, Wu S-Y, Sun T, Konofagou EE. A new brain drug delivery strategy: focused ultrasound-enhanced intranasal drug delivery. PLoS One. 2014;9(10):e108880. doi:10.1371/journal.pone.0108880.

344. Xenariou S, Liang HD, Griesenbach U, et al. Low-frequency ultrasound increases nonviral gene transfer to the mouse lung. Acta Biochim Biophys Sin (Shanghai). 2010;42(1):45-51. doi:10.1093/abbs/gmp100.

345. Gao H. Progress and perspectives on targeting nanoparticles for brain drug delivery. Acta Pharm Sin B. 2016;6(4):268-286. doi:10.1016/j.apsb.2016.05.013.

346. Cohen-Inbar O, Xu Z, Sheehan JP. Focused ultrasound-aided immunomodulation in glioblastoma multiforme: a therapeutic concept. J Ther ultrasound. 2016;4(1):2. doi:10.1186/s40349-016-0046-y.

347. Zigler M, Villares GJ, Lev DC, Melnikova VO, Bar-Eli M. Tumor Immunotherapy in Melanoma. Am J Clin Dermatol. 2008;9(5):307-311. doi:10.2165/00128071-20080905000004.

348. Millet A, Martin AR, Ronco C, Rocchi S, Benhida R. Metastatic Melanoma: Insights Into the Evolution of the Treatments and Future Challenges. Med Res Rev. August 2016. doi:10.1002/med.21404. 
349. Shi L, Chen L, Wu C, et al. PD-1 Blockade Boosts Radiofrequency Ablation-Elicited Adaptive Immune Responses against Tumor. Clin Cancer Res. 2016;22(5):1173-1184. doi:10.1158/1078-0432.CCR-15-1352.

350. Toomer $\mathrm{KH}$, Chen Z. Autoimmunity as a Double Agent in Tumor Killing and Cancer Promotion. Front Immunol. 2014;5:116. doi:10.3389/fimmu.2014.00116.

351. Systemic Therapy of Metastatic Melanoma: On the Road to Cure | Cancer Network. February 2015.

352. Zitvogel L, Kroemer G. Cancer: Antibodies regulate antitumour immunity. Nature. April 2015. doi:10.1038/nature14388.

353. Quezada SA, Peggs KS, Curran MA, Allison JP. CTLA4 blockade and GM-CSF combination immunotherapy alters the intratumor balance of effector and regulatory $T$ cells. J Clin Invest. 2006;116(7):1935-1945. doi:10.1172/JCI27745.

354. Garbe C, Eigentler TK, Keilholz U, Hauschild A, Kirkwood JM. Systematic review of medical treatment in melanoma: current status and future prospects. Oncologist. 2011;16(1):5-24. doi:10.1634/theoncologist.2010-0190.

355. Hamid O, Robert C, Daud A, et al. Safety and tumor responses with lambrolizumab (antiPD-1) in melanoma. N Engl J Med. 2013;369(2):134-144. doi:10.1056/NEJMoa1305133.

356. Robert C, Ribas A, Wolchok JD, et al. Anti-programmed-death-receptor-1 treatment with pembrolizumab in ipilimumab-refractory advanced melanoma: a randomised dosecomparison cohort of a phase 1 trial. Lancet. 2014;384(9948):1109-1117. doi:10.1016/S0140-6736(14)60958-2.

357. Liu J, Yuan Y, Chen W, et al. Immune-checkpoint proteins VISTA and PD-1 nonredundantly regulate murine T-cell responses. Proceedings of the National Academy of Sciences of the United States of America. doi:10.1073/pnas.1420370112.

358. Wang $X$, Sun J. High-intensity focused ultrasound in patients with late-stage pancreatic carcinoma. Chin Med J (Engl). 2002;115(9):1332-1335.

359. Kheirolomoom A, Ingham ES, Mahakian LM, et al. CpG expedites regression of local and systemic tumors when combined with activatable nanodelivery. J Control Release. 2015;220(Pt A):253-264. doi:10.1016/j.jconrel.2015.10.016.

360. Belizário JE. Immunodeficient Mouse Models: An Overview. Open Immunol J. 2009;2:7985.

361. Mombaerts P, Iacomini J, Johnson RS, Herrup K, Tonegawa S, Papaioannou VE. RAG1-deficient mice have no mature B and T lymphocytes. Cell. 1992;68(5):869-877.

362. Overwijk WW, Theoret MR, Finkelstein SE, et al. Tumor regression and autoimmunity after reversal of a functionally tolerant state of self-reactive CD8+ T cells. J Exp Med. 2003;198(4):569-580. doi:10.1084/jem.20030590.

363. Morris MA, Gibb DR, Picard F, Brinkmann V, Straume M, Ley K. Transient T cell accumulation in lymph nodes and sustained lymphopenia in mice treated with FTY720. European Journal of Immunology. doi:10.1002/eji.200526218. 
364. Metzler B, Gfeller P, Wieczorek G, et al. Modulation of T cell homeostasis and alloreactivity under continuous FTY720 exposure. Int Immunol. 2008;20(5):633-644. doi:10.1093/intimm/dxn023.

365. Masopust D, Choo D, Vezys V, et al. Dynamic T cell migration program provides resident memory within intestinal epithelium. J Exp Med. 2010;207(3):553-564. doi:10.1084/jem.20090858.

366. Li Y, Fang M, Zhang J, et al. Hydrogel dual delivered celecoxib and anti-PD-1 synergistically improve antitumor immunity. Oncoimmunology. 2016;5(2):e1074374. doi:10.1080/2162402X.2015.1074374.

367. Lin Z, Chen X, Li Z, et al. PD-1 Antibody Monotherapy for Malignant Melanoma: A Systematic Review and Meta-Analysis. PLoS One. 2016;11(8):e0160485. doi:10.1371/journal.pone.0160485.

368. Doedens AL, Phan AT, Stradner MH, et al. Hypoxia-inducible factors enhance the effector responses of CD8(+) T cells to persistent antigen. Nat Immunol. 2013;14(11):1173-1182. doi:10.1038/ni.2714.

369. Nizet V, Johnson RS. Interdependence of hypoxic and innate immune responses. Nat Rev Immunol. 2009;9(9):609-617. doi:10.1038/nri2607.

370. Noman MZ, Desantis G, Janji B, et al. PD-L1 is a novel direct target of HIF-1a, and its blockade under hypoxia enhanced MDSC-mediated T cell activation. J Exp Med. 2014;211(5):781-790. doi:10.1084/jem.20131916.

371. Barsoum IB, Smallwood CA, Siemens DR, et al. A mechanism of hypoxia-mediated escape from adaptive immunity in cancer cells. Cancer Res. 2014;74(3):665-674. doi:10.1158/0008-5472.CAN-13-0992.

372. Bandyopadhyay S, Quinn TJ, Scandiuzzi L, et al. Low-Intensity Focused Ultrasound Induces Reversal of Tumor-Induced T Cell Tolerance and Prevents Immune Escape. J Immunol. 2016;196(4):1964-1976. doi:10.4049/jimmunol.1500541.

373. Henao-Tamayo MI, Ordway DJ, Irwin SM, Shang S, Shanley C, Orme IM. Phenotypic definition of effector and memory T-lymphocyte subsets in mice chronically infected with Mycobacterium tuberculosis. Clin Vaccine Immunol. 2010;17(4):618-625. doi:10.1128/CVI.00368-09.

374. Selby MJ, Engelhardt JJ, Johnston RJ, et al. Preclinical Development of Ipilimumab and Nivolumab Combination Immunotherapy: Mouse Tumor Models, In Vitro Functional Studies, and Cynomolgus Macaque Toxicology. PLoS One. 2016;11(9):e0161779. doi:10.1371/journal.pone.0161779.

375. Jung S, Unutmaz D, Wong $\mathrm{P}$, et al. In vivo depletion of CD11c+ dendritic cells abrogates priming of CD8+ T cells by exogenous cell-associated antigens. Immunity. 2002;17(2):211-220.

376. Burks SR, Ziadloo A, Hancock HA, et al. Investigation of cellular and molecular responses to pulsed focused ultrasound in a mouse model. PLoS One. 2011;6(9):e24730. doi:10.1371/journal.pone.0024730. 
377. Muller WA. Transendothelial migration: unifying principles from the endothelial perspective. Immunol Rev. 2016;273(1):61-75. doi:10.1111/imr.12443.

378. Maloney E, Hwang JH. Emerging HIFU applications in cancer therapy. Int J Hyperthermia. November 2014:1-8. doi:10.3109/02656736.2014.969789. 